\title{
Construction of Enantioenriched 9H-Fluorene Frameworks via \\ Cascade Reaction Involving Remote Vinylogous Dynamic Kinetic Resolution
}

Cui-Xia Hu, ${ }^{\dagger, \S}$ Lin Chen, ${ }^{\dagger, \S}$ Di Hu, ${ }^{\dagger}$ Xue Song, ${ }^{\dagger}$ Zhi-Chao Chen, ${ }^{* \dagger}$ Wei Du, ${ }^{\dagger}$ and Ying-Chun Chen*t,*

${ }^{\dagger}$ Key Laboratory of Drug-Targeting and Drug Delivery System of the Ministry of Education and Sichuan Research Center for Drug Precision Industrial Technology, West China School of Pharmacy, Sichuan University, Chengdu 610041, China

ॠCollege of Pharmacy, Third Military Medical University, Shapingba, Chongqing 400038, China

${ }^{\S}$ C.X.H and L.C. contributed equally to this work; E-mail: chenzhichao@scu.edu.cn; ycchen@ scu.edu.cn

\section{Supporting Information}

1. General methods . .51

2. General procedure for the preparation of racemic $\alpha, \alpha$-dicyanoolefins. S1

3. General procedure for the DKR reaction of $\alpha, \alpha$-dicyanoolefins and nitroolefins .55

4. Proposed mechanism for the formation of dealkylation product $3 t$ S21

5. Transformations of the product $3 a$ .523

6. Crystal data and structural refinement for enantiopure $3 \mathrm{~m}$ and $3 \mathrm{u}$ .524

7. NMR, HRMS spectra and HPLC chromatograms S27 


\section{General methods}

Unless otherwise noted, the reactions were carried out under ambient atmosphere; when the reactions required heating, the heat source was oil bath. ${ }^{1} \mathrm{H}$ NMR (400 or $\left.600 \mathrm{MHz}\right),{ }^{13} \mathrm{C}$ NMR (100 or $150 \mathrm{MHz}$ ) and ${ }^{19} \mathrm{~F}$ NMR (376 MHz) spectra were recorded on Varian INOVA-400/54, Agilent DD2-600/54 or Bruker AscendTM 400 instruments (Chemical shifts were reported in ppm from tetramethylsilane with the solvent resonance as the internal standard in $\mathrm{CDCl}_{3}$ solution, unless otherwise noted). The following abbreviations were used to explain the multiplicities: $s=$ singlet, $d$ $=$ doublet, $\mathrm{t}=$ triplet, $\mathrm{q}=$ quartet, $\mathrm{td}=$ triple doublet, $\mathrm{dt}=$ double triplet, $\mathrm{m}=$ multiplet, and coupling constants $(J)$ are reported in Hertz $(\mathrm{Hz})$. High resolution mass spectra (HRMS) were recorded on a Waters SYNAPT G2, Agilent G1969-85000 or Shimadzu LCMS-IT-TOF using a time-of-flight mass spectrometer equipped with electrospray ionization (ESI) source. X-ray diffraction experiments were carried out on an Agilent Gemini and the data obtained were deposited at the Cambridge Crystallographic Data Centre. In each case, diastereomeric ratio (if any) was determined by ${ }^{1} \mathrm{H}$ NMR analysis and enantiomeric excess was determined by HPLC analysis on a chiral stationary phase in comparison with the authentic racemate, using a Daicel Chiralpak AD-H Column $(250 \times 4.6 \mathrm{~mm})$, Chiralpak IB Column $(250 \times 4.6 \mathrm{~mm})$, Chiralpak IC Column $(250 \times 4.6 \mathrm{~mm})$, Chiralpak ID Column $(250 \times 4.6 \mathrm{~mm})$, Chiralpak IE Column $(250 \times 4.6 \mathrm{~mm})$ or Chiralpak IG Column $(250 \times 4.6 \mathrm{~mm})$. UV detection was monitored at $254 \mathrm{~nm}$. Optical rotation was measured in $\mathrm{MeOH}$ or $\mathrm{CHCl}_{3}$ solution at $25^{\circ} \mathrm{C}$. Column chromatography was performed on silica gel (200-300 mesh) eluting with redistilled EtOAc and petroleum ether (PE). TLC was performed on glass-backed silica plates. UV light (monitored at $254 \mathrm{~nm}$ ), $\mathrm{I}_{2}$ and solution of potassium permanganate were used to visualize products or starting materials. All chemicals were used without purification as commercially available unless otherwise noted. Nitroolefins ${ }^{1}$ and chiral catalysts ${ }^{2}$ were prepared according to the literature procedures.

(1) Wu, M.-Y.; Wang, M.-Q.; Li, K.; Feng, X.-W.; He, T.; Wang, N.; Yu, X.-Q. Tetrahedron Lett. 2011, 52,679 .

(2) Okino, T.; Hoashi, Y.; Takemoto, Y. J. Am. Chem. Soc. 2003, 125, 12672.

\section{General procedure for the preparation of racemic $\alpha, \alpha$-dicyanoolefins}

$\alpha, \alpha$-Dicyanoolefins 1 were prepared according to the following general procedure: 
1a as an example:

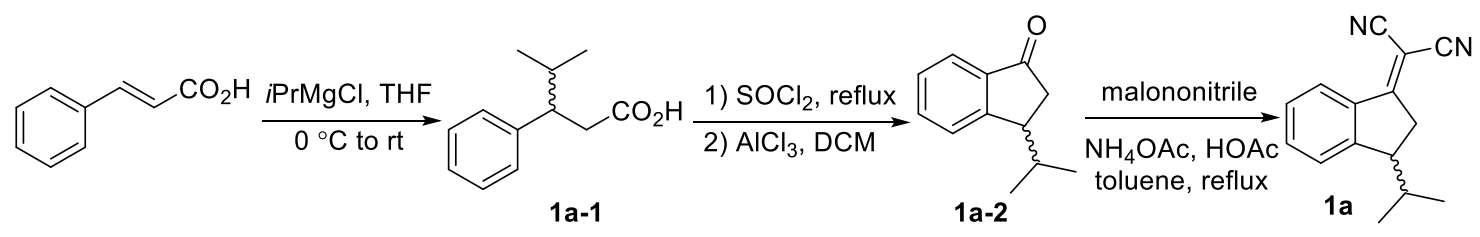

The cinnamic acid (2.96 g, $20.0 \mathrm{mmol})$ was dissolved in THF $(60 \mathrm{~mL})$ and cooled to $0{ }^{\circ} \mathrm{C}$. To the mixture was added $i \mathrm{PrMgCl}$ in THF (2.0 M, $35 \mathrm{~mL}, 70 \mathrm{mmol}, 3.5$ equiv) dropwise. The resulting mixture was warmed to rt and stirred overnight. After completion, the mixture was quenched with diluted $\mathrm{HCl}$ solution $(3.0 \mathrm{M}, 50 \mathrm{~mL})$, and extracted by EtOAc $(50 \mathrm{~mL})$, washed with brine, and dried over $\mathrm{Na}_{2} \mathrm{SO}_{4}$. After concentration, the residue was purified by column chromatography $(\mathrm{PE} / \mathrm{EtOAc}=$ 3:1) to give 1a-1 as a colorless oil (1.90 g, 50\% yield).

1a-1 (1.90 g, $9.88 \mathrm{mmol})$ was added to $\mathrm{SOCl}_{2}(20.0 \mathrm{~mL})$, and 1 drop of DMF was added. The mixture was heated to reflux for $2 \mathrm{~h}$, then the excess $\mathrm{SOCl}_{2}$ was evaporated carefully. The residue was dissolved in $\mathrm{DCM}(20 \mathrm{~mL})$, and $\mathrm{AlCl}_{3}(1.45 \mathrm{~g}, 10.9 \mathrm{mmol}, 1.2$ equiv) was added in portions. The mixture was stirred at rt overnight, and poured into ice water. The organic layer was isolated, and the aqueous phase was extracted with DCM $(3 \times 10 \mathrm{~mL})$. The organic phases were combined, washed with brine and dried over $\mathrm{Na}_{2} \mathrm{SO}_{4}$. After concentration, the residue was purified by column chromatography $(\mathrm{PE} / \mathrm{EtOAc}=20: 1)$ to give 1a-2 as a light red oil $(1.40 \mathrm{~g}, 81 \%$ yield $)$.

1a-2 (1.40 g, $8.03 \mathrm{mmol}$ ), malononitrile (3.70 g, $56.0 \mathrm{mmol}, 7$ equiv), $\mathrm{NH}_{4} \mathrm{OAc}(4.32 \mathrm{~g}, 56.0$ mmol, 7 equiv) and HOAc ( $4.80 \mathrm{~g}, 80.0 \mathrm{mmol}, 10$ equiv) were added to toluene ( $80 \mathrm{~mL})$. The mixture was heated to reflux for 2 days. Then it was cooled to rt, and water (50 mL) and EtOAC (50 mL) were added. The mixture was filtered through a pad of celite, and the organic phase was isolated. The aqueous phase was extracted with EtOAc $(3 \times 10 \mathrm{~mL})$. The organic phases were combined and dried over $\mathrm{Na}_{2} \mathrm{SO}_{4}$. After concentration, the residue was purified by column chromatography $(\mathrm{PE} / \mathrm{EtOAc}=$ 20:1) to give 1a as a pale yellow solid (1.2 $\mathrm{g}, 67 \%$ yield).

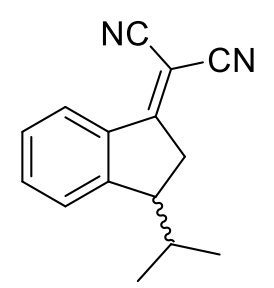

2-(3-Isopropyl-2,3-dihydro-1H-inden-1-ylidene)malononitrile (1a): yellow solid; m.p. $98-101{ }^{\circ} \mathrm{C} ;{ }^{1} \mathrm{H}$ NMR $\left(400 \mathrm{MHz}, \mathrm{CDCl}_{3}\right) \delta(\mathrm{ppm}) 8.38(\mathrm{~d}, J=8.1 \mathrm{~Hz}, 1 \mathrm{H})$, $7.73-7.58(\mathrm{~m}, 1 \mathrm{H}), 7.53-7.38(\mathrm{~m}, 2 \mathrm{H}), 3.43-3.40(\mathrm{~m}, 1 \mathrm{H}), 3.23(\mathrm{dd}, J=20.0,7.3$ $\mathrm{Hz}, 1 \mathrm{H}), 3.01(\mathrm{dd}, J=20.0,2.8 \mathrm{~Hz}, 1 \mathrm{H}), 2.28-2.20(\mathrm{~m}, 1 \mathrm{H}), 1.03(\mathrm{~d}, J=6.9 \mathrm{~Hz}$, $3 \mathrm{H}), 0.66(\mathrm{~d}, J=6.8 \mathrm{~Hz}, 3 \mathrm{H}) ;{ }^{13} \mathrm{C} \mathrm{NMR}\left(100 \mathrm{MHz}, \mathrm{CDCl}_{3}\right) \delta(\mathrm{ppm}) 178.8,156.7,135.9,135.1,128.3$, 125.9, 125.7, 113.6, 113.0, 74.1, 47.9, 36.9, 31.6, 20.8, 16.5; HRMS (ESI-TOF) m/z: $[\mathrm{M}+\mathrm{Na}]^{+}$Calcd 
for $\left[\mathrm{C}_{15} \mathrm{H}_{14} \mathrm{~N}_{2}+\mathrm{Na}\right]^{+}$245.1049; Found 245.1053.

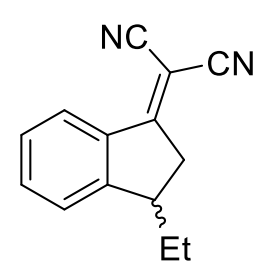

2-(3-Ethyl-2,3-dihydro-1H-inden-1-ylidene)malononitrile (1b): white solid; m.p. 75-77 ${ }^{\circ} \mathrm{C} ;{ }^{1} \mathrm{H}$ NMR $\left(400 \mathrm{MHz}, \mathrm{CDCl}_{3}\right) \delta(\mathrm{ppm}) 8.36(\mathrm{~d}, J=8.1 \mathrm{~Hz}, 1 \mathrm{H})$, 7.70-7.58 (m, 1H), 7.50-7.46 (m, 1H), 7.45-7.42 (m, 1H), $3.42(\mathrm{dd}, J=19.5,7.1$

$\mathrm{Hz}, 1 \mathrm{H}), 3.37-3.31(\mathrm{~m}, 1 \mathrm{H}), 2.91(\mathrm{dd}, J=19.5,2.7 \mathrm{~Hz}, 1 \mathrm{H}), 2.00-1.89(\mathrm{~m}, 1 \mathrm{H})$, 1.66-1.43 (m, 1H), $0.99(\mathrm{t}, J=7.4 \mathrm{~Hz}, 3 \mathrm{H}) ;{ }^{13} \mathrm{C} \mathrm{NMR}\left(100 \mathrm{MHz}, \mathrm{CDCl}_{3}\right) \delta(\mathrm{ppm}) 178.4,157.5$, 135.3, 135.2, 128.3, 125.9, 125.5, 113.6, 112.9, 74.3, 43.3, 40.8, 28.4, 11.3; HRMS (ESI-TOF) m/z: $[\mathrm{M}+\mathrm{Na}]^{+}$Calcd for $\left[\mathrm{C}_{14} \mathrm{H}_{12} \mathrm{~N}_{2}+\mathrm{Na}\right]^{+}$231.0893; Found 231.0896.

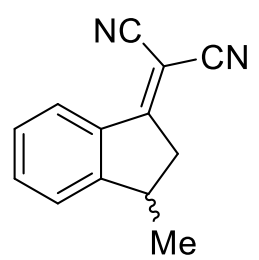

2-(3-Methyl-2,3-dihydro-1H-inden-1-ylidene)malononitrile (1c): white solid; m.p. $119-121{ }^{\circ} \mathrm{C} ;{ }^{1} \mathrm{H}$ NMR $\left(400 \mathrm{MHz}, \mathrm{CDCl}_{3}\right) \delta$ (ppm) $8.37(\mathrm{~d}, J=8.0 \mathrm{~Hz}, 1 \mathrm{H})$, 7.67-7.60 (m, 1H), 7.49-7.40 (m, 2H), 3.58-3.41 (m, 2H), $2.82(\mathrm{dd}, J=19.3,2.8$ $\mathrm{Hz}, 1 \mathrm{H}), 1.39(\mathrm{~d}, J=6.9 \mathrm{~Hz}, 3 \mathrm{H}) ;{ }^{13} \mathrm{C} \mathrm{NMR}\left(100 \mathrm{MHz}, \mathrm{CDCl}_{3}\right) \delta(\mathrm{ppm}) 178.1$, 158.6, 135.3 134.8, 128.3 125.9, 125.1, 113.5, 112.9, 74.4, 43.5, 36.6, 20.8; HRMS (ESI-TOF) m/z: $[\mathrm{M}-\mathrm{H}]^{-}$Calcd for $\left[\mathrm{C}_{13} \mathrm{H}_{10} \mathrm{~N}_{2}-\mathrm{H}\right]^{-}$193.0771; Found 193.0772.

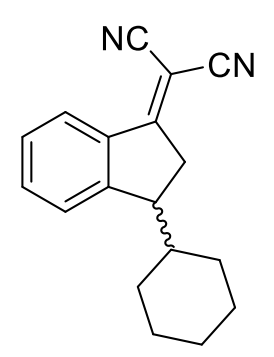

2-(3-Cyclohexyl-2,3-dihydro-1H-inden-1-ylidene)malononitrile $\quad(1 d)$ : white solid; m.p. $103-105{ }^{\circ} \mathrm{C} ;{ }^{1} \mathrm{H}$ NMR $\left(400 \mathrm{MHz}, \mathrm{CDCl}_{3}\right) \delta$ (ppm) 8.37 (d, $J=8.1 \mathrm{~Hz}$, 1H), 7.66-7.55 (m, 1H), 7.51-7.38 (m, 2H), 3.39-3.35 (m, 1H), 3.23 (dd, J=19.9, $7.3 \mathrm{~Hz}, 1 \mathrm{H}), 3.07(\mathrm{dd}, J=19.9,2.6 \mathrm{~Hz}, 1 \mathrm{H}), 1.85-1.76(\mathrm{~m}, 2 \mathrm{H}), 1.73-1.67(\mathrm{~m}, 2 \mathrm{H})$, 1.31-1.27 (m, 1H), 1.22-1.04 (m, 4H), 0.93-0.82 (m, 2H); ${ }^{13} \mathrm{C}$ NMR (100 MHz, $\left.\mathrm{CDCl}_{3}\right) \delta(\mathrm{ppm}) 178.9,156.5,135.9,135.0,128.2,125.9,125.9,113.7,113.0,74.0,47.6,42.2,38.0$, 31.3, 27.2, 26.5, 26.2, 26.1; HRMS (ESI-TOF) $\mathrm{m} / \mathrm{z}:[\mathrm{M}+\mathrm{Na}]^{+} \mathrm{Calcd}$ for $\left[\mathrm{C}_{18} \mathrm{H}_{18} \mathrm{~N}_{2}+\mathrm{Na}\right]^{+} 285.1362$; Found 285.1363.

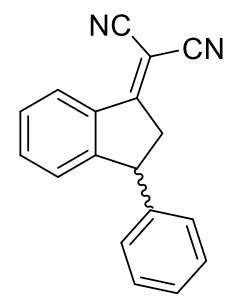

2-(3-Phenyl-2,3-dihydro-1H-inden-1-ylidene)malononitrile (1e): white solid; m.p. 136-138 ${ }^{\circ} \mathrm{C} ;{ }^{1} \mathrm{H}$ NMR $\left(400 \mathrm{MHz}, \mathrm{CDCl}_{3}\right) \delta(\mathrm{ppm}) 8.44(\mathrm{~d}, J=8.0 \mathrm{~Hz}, 1 \mathrm{H}), 7.58(\mathrm{t}, J$ $=7.5 \mathrm{~Hz}, 1 \mathrm{H}), 7.48(\mathrm{t}, J=7.7 \mathrm{~Hz}, 1 \mathrm{H}), 7.40-7.19(\mathrm{~m}, 4 \mathrm{H}), 7.17-7.00(\mathrm{~m}, 2 \mathrm{H}), 4.57$ $(\mathrm{dd}, J=7.7,3.7 \mathrm{~Hz}, 1 \mathrm{H}), 3.81(\mathrm{dd}, J=19.9,7.6 \mathrm{~Hz}, 1 \mathrm{H}), 3.22(\mathrm{dd}, J=19.9,3.7 \mathrm{~Hz}$, 
$1 \mathrm{H}) ;{ }^{13} \mathrm{C}$ NMR $\left(100 \mathrm{MHz}, \mathrm{CDCl}_{3}\right) \delta(\mathrm{ppm}) 177.7,156.8,142.3,135.6,135.5,129.3,128.8,127.7$, 126.9, 125.9, 113.4, 112.9, 74.9, 48.1, 45.1; HRMS (ESI-TOF) m/z: $[\mathrm{M}-\mathrm{H}]^{-}$Calcd for $\left[\mathrm{C}_{18} \mathrm{H}_{11} \mathrm{~N}_{2}\right.$ $-\mathrm{H}]^{-}$255.0927; Found 255.0928.

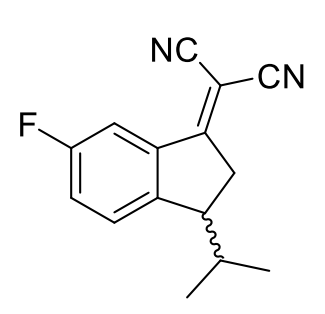

2-(6-Fluoro-3-isopropyl-2,3-dihydro-1H-inden-1-ylidene)malononitrile (1f): yellow solid; m.p. $94-96{ }^{\circ} \mathrm{C} ;{ }^{1} \mathrm{H}$ NMR $\left(400 \mathrm{MHz}, \mathrm{CDCl}_{3}\right) \delta(\mathrm{ppm}) 8.03$ (dd, $J=$ 8.9, 2.4 Hz, 1H), $7.45(\mathrm{dd}, J=8.6,5.0 \mathrm{~Hz}, 1 \mathrm{H}), 7.37-7.32(\mathrm{~m}, 1 \mathrm{H}), 3.41-3.38$ $(\mathrm{m}, 1 \mathrm{H}), 3.27(\mathrm{dd}, J=20.1,7.2 \mathrm{~Hz}, 1 \mathrm{H}), 3.04(\mathrm{dd}, J=20.0,2.6 \mathrm{~Hz}, 1 \mathrm{H})$, 2.26-2.18 (m, 1H), $1.02(\mathrm{~d}, J=6.8 \mathrm{~Hz}, 3 \mathrm{H}), 0.68(\mathrm{~d}, J=6.9 \mathrm{~Hz}, 3 \mathrm{H}) ;{ }^{13} \mathrm{C} \mathrm{NMR}\left(100 \mathrm{MHz}, \mathrm{CDCl}_{3}\right)$ $\delta(\mathrm{ppm}) 177.6\left(\mathrm{~d},{ }^{3} J_{\mathrm{FC}}=3.7 \mathrm{~Hz}\right), 162.2\left(\mathrm{~d},{ }^{1} J_{\mathrm{FC}}=246.8 \mathrm{~Hz}\right), 152.3\left(\mathrm{~d},{ }^{3} J_{\mathrm{FC}}=2.2 \mathrm{~Hz}\right), 137.2,137.1$, $127.0\left(\mathrm{~d},{ }^{3} J_{\mathrm{FC}}=8.6 \mathrm{~Hz}\right), 122.9\left(\mathrm{~d},{ }^{2} J_{\mathrm{FC}}=23.3 \mathrm{~Hz}\right), 113.2,112.4,111.9\left(\mathrm{~d},{ }^{2} J_{\mathrm{FC}}=23.8 \mathrm{~Hz}\right), 75.5,47.4$, 37.4, 31.7, 20.7, 16.5; ${ }^{19} \mathrm{~F}$ NMR (376 MHz, $\left.\mathrm{CDCl}_{3}\right) \delta$ (ppm) -111.7; HRMS (ESI-TOF) m/z: [M + $\mathrm{Na}]^{+}$Calcd for $\left[\mathrm{C}_{15} \mathrm{H}_{13} \mathrm{FN}_{2}+\mathrm{Na}\right]^{+}$263.0955; Found 263.0963.

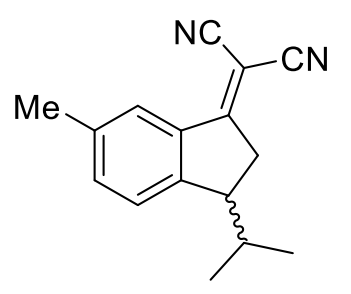

\section{2-(3-Isopropyl-6-methyl-2,3-dihydro-1H-inden-1-ylidene)malononitrile} (1g): yellow solid; m.p. 101-103 ${ }^{\circ} \mathrm{C} ;{ }^{1} \mathrm{H} \mathrm{NMR}\left(400 \mathrm{MHz}, \mathrm{CDCl}_{3}\right) \delta$ (ppm) 8.15 (s, 1H), 7.49-7.40 (m, 1H), $7.35(\mathrm{~d}, J=7.9 \mathrm{~Hz}, 1 \mathrm{H}), 3.38-3.34(\mathrm{~m}, 1 \mathrm{H}), 3.21$ (dd, $J=19.9,7.2 \mathrm{~Hz}, 1 \mathrm{H}), 2.98(\mathrm{dd}, J=19.9,2.7 \mathrm{~Hz}, 1 \mathrm{H}), 2.43(\mathrm{~s}, 3 \mathrm{H})$, 2.24-2.16 (m, 1H), $1.01(\mathrm{~d}, J=6.9 \mathrm{~Hz}, 3 \mathrm{H}), 0.66(\mathrm{~d}, J=6.8 \mathrm{~Hz}, 3 \mathrm{H}) ;{ }^{13} \mathrm{C} \mathrm{NMR}\left(100 \mathrm{MHz}, \mathrm{CDCl}_{3}\right)$ $\delta(\mathrm{ppm}) 178.8,154.2,138.4,136.5,136.1,125.7,125.4,113.8,113.1,73.6,47.5,37.2,31.6,21.4$, 20.7, 16.5; HRMS (ESI-TOF) m/z: $[\mathrm{M}+\mathrm{Na}]^{+}$Calcd for $\left[\mathrm{C}_{16} \mathrm{H}_{16} \mathrm{~N}_{2}+\mathrm{Na}\right]^{+}$259.1206; Found 259.1213.

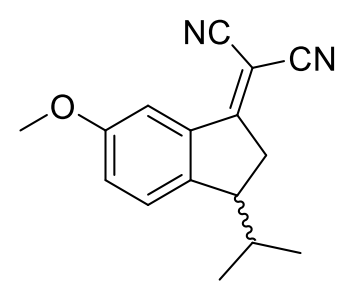

\section{2-(3-Isopropyl-6-methoxy-2,3-dihydro- $1 H$-inden-1-ylidene)malononitrile}

(1h): yellow solid; m.p. $101-103^{\circ} \mathrm{C} ;{ }^{1} \mathrm{H}$ NMR $\left(400 \mathrm{MHz}, \mathrm{CDCl}_{3}\right) \delta(\mathrm{ppm}) 7.83$ $(\mathrm{d}, J=2.4 \mathrm{~Hz}, 1 \mathrm{H}), 7.35(\mathrm{~d}, J=8.6 \mathrm{~Hz}, 1 \mathrm{H}), 7.21(\mathrm{dd}, J=8.6,2.4 \mathrm{~Hz}, 1 \mathrm{H})$, $3.86(\mathrm{~s}, 3 \mathrm{H}), 3.36-3.33(\mathrm{~m}, 1 \mathrm{H}), 3.23(\mathrm{dd}, J=19.9,7.1 \mathrm{~Hz}, 1 \mathrm{H}), 2.99(\mathrm{dd}, J=$ $19.9,2.5 \mathrm{~Hz}, 1 \mathrm{H}), 2.22-2.14(\mathrm{~m}, 1 \mathrm{H}), 1.00(\mathrm{~d}, J=6.9 \mathrm{~Hz}, 3 \mathrm{H}), 0.66(\mathrm{~d}, J=6.9 \mathrm{~Hz}, 3 \mathrm{H}) ;{ }^{13} \mathrm{C} \mathrm{NMR}$ $\left(100 \mathrm{MHz}, \mathrm{CDCl}_{3}\right) \delta(\mathrm{ppm}) 178.9,159.6,149.7,136.9,126.3,124.3,113.7,113.1,107.3,73.7,55.6$, 47.3, 37.5, 31.6, 20.7, 16.6; HRMS (ESI-TOF) m/z: $[\mathrm{M}+\mathrm{Na}]^{+}$Calcd for $\left[\mathrm{C}_{16} \mathrm{H}_{16} \mathrm{~N}_{2} \mathrm{O}+\mathrm{Na}\right]^{+} 275.1155$; Found 275.1159. 
<smiles>Cc1ccc2c(c1)C(=C(C#N)C#N)CC2C(C)C</smiles>

2-(3-Isopropyl-5-methyl-2,3-dihydro-1H-inden-1-ylidene)malononitrile (1i): white solid; m.p. $91-93{ }^{\circ} \mathrm{C} ;{ }^{1} \mathrm{H}$ NMR $\left(400 \mathrm{MHz}, \mathrm{CDCl}_{3}\right) \delta(\mathrm{ppm}) 8.25$ (d, $J=9.0 \mathrm{~Hz}, 1 \mathrm{H}), 7.25(\mathrm{~s}, 1 \mathrm{H}), 7.24(\mathrm{~d}, J=7.7 \mathrm{~Hz}, 1 \mathrm{H}), 3.37-3.34(\mathrm{~m}, 1 \mathrm{H}), 3.20$ (dd, $J=19.9,7.3 \mathrm{~Hz}, 1 \mathrm{H}), 2.98(\mathrm{dd}, J=19.9,2.8 \mathrm{~Hz}, 1 \mathrm{H}), 2.47$ (s, 3H), $2.26-2.18(\mathrm{~m}, 1 \mathrm{H}), 1.02(\mathrm{~d}, J=6.9 \mathrm{~Hz}, 3 \mathrm{H}), 0.65(\mathrm{~d}, J=6.9 \mathrm{~Hz}, 3 \mathrm{H}) ;{ }^{13} \mathrm{C} \mathrm{NMR}$ $\left(100 \mathrm{MHz}, \mathrm{CDCl}_{3}\right) \delta(\mathrm{ppm}) 178.6,157.2,146.8,133.6,129.6,126.1,125.7,113.9,113.3,72.7,47.7$, 36.9, 31.5, 22.3, 20.8, 16.4; HRMS (ESI-TOF) m/z: $[\mathrm{M}+\mathrm{Na}]^{+}$Calcd for $\left[\mathrm{C}_{16} \mathrm{H}_{16} \mathrm{~N}_{2}+\mathrm{Na}\right]^{+} 259.1206$; Found 259.1206.<smiles>N#CC(C#N)=C1CCC(C2CCCCC2)c2ccccc21</smiles>

(4-Cyclohexyl-3,4-dihydronaphthalen-1(2H)-ylidene)malononitrile (4): white solid; m.p. $113-115{ }^{\circ} \mathrm{C} ;{ }^{1} \mathrm{H}$ NMR $\left(400 \mathrm{MHz}, \mathrm{CDCl}_{3}\right) \delta(\mathrm{ppm}) 8.10(\mathrm{dd}, J=7.9,1.3$ $\mathrm{Hz}, 1 \mathrm{H}), 7.49(\mathrm{td}, J=7.5,1.3 \mathrm{~Hz}, 1 \mathrm{H}), 7.36(\mathrm{td}, J=7.7,1.3 \mathrm{~Hz}, 1 \mathrm{H}), 7.26-7.23(\mathrm{~m}$, 1H), 3.20-3.07 (m, 1H), 2.9-2.87 (m, 1H), 2.66-2.56 (m, 1H), 2.23-2.13 (m, 1H), $1.88-1.60(\mathrm{~m}, 5 \mathrm{H}), 1.52-1.43(\mathrm{~m}, 1 \mathrm{H}), 1.33-1.08(\mathrm{~m}, 4 \mathrm{H}), 1.06-0.87(\mathrm{~m}, 2 \mathrm{H}) ;{ }^{13} \mathrm{C}$ NMR (100 MHz, $\left.\mathrm{CDCl}_{3}\right) \delta(\mathrm{ppm}) 172.7,145.9,133.1,129.8,129.6,128.3,127.0,113.9,113.3,45.4$, 39.7, 31.7, 30.5, 29.6, 26.19, 26.17, 26.1, 21.8; HRMS (ESI-TOF) m/z: $[\mathrm{M}-\mathrm{H}]^{-}$Calcd for $\left[\mathrm{C}_{19} \mathrm{H}_{20} \mathrm{~N}_{2}\right.$ $-\mathrm{H}]^{-}$275.1553; Found 275.1555.

\section{General procedure for the DKR reaction of $\alpha, \alpha$-dicyanoolefins and nitroolefins}

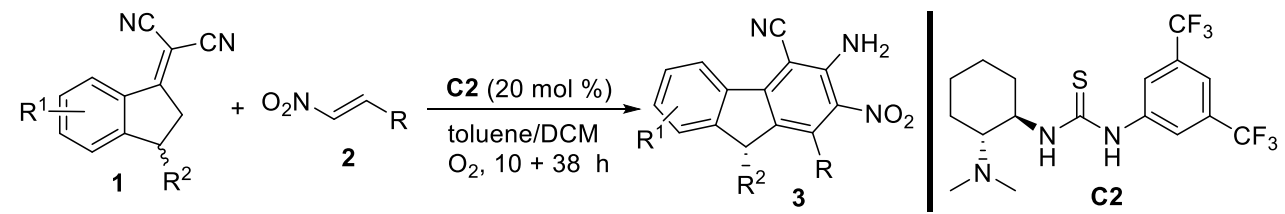

$\alpha, \alpha$-Dicyanoolefin 1 ( $0.1 \mathrm{mmol})$, nitroolefin 2 (0.2 mmol, 2.0 equiv) and catalyst $\mathbf{C 2}$ (0.02 mmol, $20 \mathrm{~mol} \%$ ) were added to a reaction tube, and the mixture was degassed and backfilled with oxygen (balloon). Dry toluene $(1.0 \mathrm{~mL})$ was added via syringe. The mixture was stirred at $\mathrm{rt}$ for $10 \mathrm{~h}$. After the consumption of substrate 1, dry DCM $(1.0 \mathrm{~mL})$ was added via syringe, and the mixture was stirred for another $38 \mathrm{~h}$. After completion (monitored by TLC), the solvent was evaporated in vacuo. The residue was purified by flash chromatography on silica gel (PE/EtOAc) to give the product 3 . 


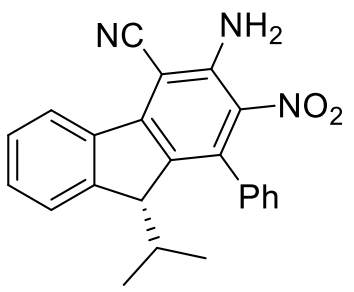

(R)-3-Amino-9-isopropyl-2-nitro-1-phenyl-9H-fluorene-4-carbonitrile

(3a): 2-(3-Isopropyl-2,3-dihydro-1H-inden-1-ylidene)malononitrile 1a (22.2 mg, $0.0999 \mathrm{mmol}), \beta$-nitrostyrene $\mathbf{2 a}(29.8 \mathrm{mg}, 0.200 \mathrm{mmol}, 2.0$ equiv) and C2 $(8.3 \mathrm{mg}, 0.020 \mathrm{mmol}, 20 \mathrm{~mol} \%)$ were added to a reaction tube, and the mixture was degassed and backfilled with oxygen (balloon). Dry toluene $(1.0 \mathrm{~mL})$ was added via syringe, and the mixture was stirred at $\mathrm{rt}$ for $10 \mathrm{~h}$. After the consumption of compound 1a, dry DCM $(1.0 \mathrm{~mL})$ was added via syringe, and the mixture was stirred for another $38 \mathrm{~h}$. After completion (monitored by TLC), the solvent was evaporated in vacuo. The residue was purified by flash chromatography on silica gel $(\mathrm{PE} / \mathrm{EtOAc}=20: 1)$ to give the product 3a: $29.9 \mathrm{mg}(0.0809 \mathrm{mmol})$, as a yellow solid, $81 \%$ yield; m.p. $236-238^{\circ} \mathrm{C} ;[\alpha]_{\mathrm{D}}{ }^{25}=-322(c=0.027 \mathrm{in} \mathrm{MeOH}), 94 \%$ ee, determined by HPLC analysis [Chiralpak IB, $n$-hexane $/ i-\mathrm{PrOH}=80 / 20,1.0 \mathrm{~mL} / \mathrm{min}, \lambda=254 \mathrm{~nm}$, $\mathrm{t}$ (minor) $=$ $6.73 \mathrm{~min}, \mathrm{t}$ (major) $=7.05 \mathrm{~min}] ;{ }^{1} \mathrm{H} \mathrm{NMR}\left(400 \mathrm{MHz}, \mathrm{CDCl}_{3}\right) \delta(\mathrm{ppm}) 8.48(\mathrm{~d}, J=7.6 \mathrm{~Hz}, 1 \mathrm{H})$, 7.55-7.42 (m, 7H), $7.17(\mathrm{~d}, J=6.5 \mathrm{~Hz}, 1 \mathrm{H}), 5.76(\mathrm{brs}, 2 \mathrm{H}), 4.11(\mathrm{~d}, J=2.7 \mathrm{~Hz}, 1 \mathrm{H}), 1.57-1.54(\mathrm{~m}$, $1 \mathrm{H}), 0.97(\mathrm{~d}, J=7.0 \mathrm{~Hz}, 3 \mathrm{H}),-0.05(\mathrm{~d}, J=6.8 \mathrm{~Hz}, 3 \mathrm{H}) ;{ }^{13} \mathrm{C} \mathrm{NMR}\left(100 \mathrm{MHz}, \mathrm{CDCl}_{3}\right) \delta(\mathrm{ppm}) 147.2$, $145.9,143.1,137.8,136.7,135.0,134.5,133.6,128.6,128.0,127.7,126.83,126.76,125.7,124.7$, 122.0, 114.7, 89.1, 51.7, 27.5, 20.9, 13.8; HRMS (ESI-TOF) $\mathrm{m} / \mathrm{z}:[\mathrm{M}+\mathrm{Na}]^{+}$Calcd for $\left[\mathrm{C}_{23} \mathrm{H}_{19} \mathrm{~N}_{3} \mathrm{O}_{2}\right.$ $+\mathrm{Na}]^{+}$392.1370; Found 392.1364.

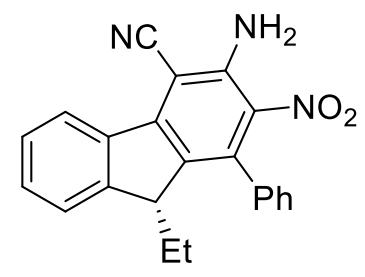

(R)-3-Amino-9-ethyl-2-nitro-1-phenyl-9H-fluorene-4-carbonitrile $\quad(3 \mathrm{~b})$ : 2-(3-Ethyl-2,3-dihydro-1H-inden-1-ylidene)malononitrile $\quad \mathbf{1 b} \quad(20.8 \quad \mathrm{mg}$, $0.0999 \mathrm{mmol}), \beta$-nitrostyrene $\mathbf{2 a}(29.8 \mathrm{mg}, 0.200 \mathrm{mmol}, 2.0$ equiv) and $\mathbf{C 2}$ (8.3 $\mathrm{mg}, 0.020 \mathrm{mmol}, 20 \mathrm{~mol} \%$ ) were added to a reaction tube, and the mixture was degassed and backfilled with oxygen (balloon). Dry toluene (1.0 mL) was added via syringe, and the mixture was stirred at $\mathrm{rt}$ for $10 \mathrm{~h}$. After the consumption of compound $\mathbf{1 b}$, dry DCM $(1.0 \mathrm{~mL})$ was added via syringe, and the mixture was stirred for another $38 \mathrm{~h}$. After completion (monitored by TLC), the solvent was evaporated in vacuo. The residue was purified by flash chromatography on silica gel $(\mathrm{PE} / \mathrm{EtOAc}=20: 1)$ to give the product $3 \mathbf{b}: 28.7 \mathrm{mg}(0.0808 \mathrm{mmol})$, as a yellow solid, $81 \%$ yield; m.p. $203-205^{\circ} \mathrm{C} ;[\alpha]_{\mathrm{D}}{ }^{25}=-90.9(c=0.022$ in $\mathrm{MeOH}), 87 \%$ ee, determined by HPLC analysis [Chiralpak AD-H, $n$-hexane $/ i$-PrOH $=90 / 10,1.0 \mathrm{~mL} / \mathrm{min}, \lambda=254 \mathrm{~nm}, \mathrm{t}(\operatorname{minor})=$ $7.51 \mathrm{~min}, \mathrm{t}$ (major) = 8.77 min]; ${ }^{1} \mathrm{H} \mathrm{NMR}\left(400 \mathrm{MHz}, \mathrm{CDCl}_{3}\right) \delta(\mathrm{ppm}) 8.47-8.45(\mathrm{~m}, 1 \mathrm{H}), 7.55-7.40$ 
(m, 7H), $7.17(\mathrm{~d}, J=6.9 \mathrm{~Hz}, 1 \mathrm{H}), 5.79(\mathrm{brs}, 2 \mathrm{H}), 4.15(\mathrm{dd}, J=5.0,3.8 \mathrm{~Hz}, 1 \mathrm{H}), 1.68-1.59(\mathrm{~m}, 1 \mathrm{H})$, $1.06-0.97(\mathrm{~m}, 1 \mathrm{H}), 0.11(\mathrm{t}, J=7.4 \mathrm{~Hz}, 3 \mathrm{H}) ;{ }^{13} \mathrm{C} \mathrm{NMR}\left(100 \mathrm{MHz}, \mathrm{CDCl}_{3}\right) \delta(\mathrm{ppm}) 149.4,146.3,143.3$, $138.3,136.2,134.6,133.9,133.3,129.3,128.0,127.9,127.6,126.9,126.8,125.5,123.1,122.0,114.7$, 89.4, 46.4, 21.9, 6.4; HRMS (ESI-TOF) m/z: $[\mathrm{M}+\mathrm{Na}]^{+}$Calcd for $\left[\mathrm{C}_{22} \mathrm{H}_{17} \mathrm{~N}_{3} \mathrm{O}_{2}+\mathrm{Na}\right]^{+} 378.1213$; Found 378.1210 .

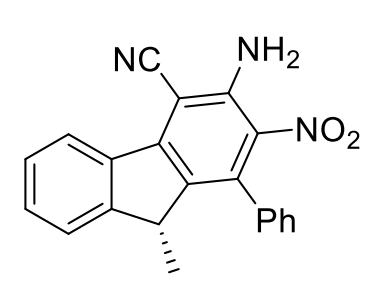

(R)-3-Amino-9-methyl-2-nitro-1-phenyl-9H-fluorene-4-carbonitrile (3c): 2-(3-Methyl-2,3-dihydro-1H-inden-1-ylidene)malononitrile $1 c \quad(19.4 \mathrm{mg}$, $0.0999 \mathrm{mmol}), \beta$-nitrostyrene $\mathbf{2 a}(29.8 \mathrm{mg}, 0.200 \mathrm{mmol}, 2.0$ equiv) and C6 (7.8 $\mathrm{mg}, 0.020 \mathrm{mmol}, 20 \mathrm{~mol} \%$ ) were added to a reaction tube, and the mixture was degassed and backfilled with oxygen (balloon). Dry toluene (1.0 mL) was added via syringe, and the mixture was stirred at $\mathrm{rt}$ for $10 \mathrm{~h}$. After the consumption of compound 1c, dry DCM $(1.0 \mathrm{~mL})$ was added via syringe, and the mixture was stirred for another $38 \mathrm{~h}$. After completion (monitored by TLC), the solvent was evaporated in vacuo. The residue was purified by flash chromatography on silica gel $(\mathrm{PE} / \mathrm{EtOAc}=20: 1)$ to give the product $3 \mathrm{c}: 17.6 \mathrm{mg}(0.0516 \mathrm{mmol})$, as a pale yellow solid, 52\% yield; m.p. $201-203{ }^{\circ} \mathrm{C}$; $[\alpha]_{\mathrm{D}}{ }^{25}=+61.0\left(c=0.2\right.$ in $\left.\mathrm{CHCl}_{3}\right), 24 \%$ ee, determined by HPLC analysis [Chiralpak AD-H, $n$-hexane $/ i$-PrOH $=80 / 20,1.0 \mathrm{~mL} / \mathrm{min}, \lambda=254 \mathrm{~nm}$, $\mathrm{t}($ major $)=5.48 \mathrm{~min}, \mathrm{t}($ minor $)=6.52 \mathrm{~min}] ;{ }^{1} \mathrm{H} \mathrm{NMR}\left(400 \mathrm{MHz}, \mathrm{CDCl}_{3}\right) \delta(\mathrm{ppm}) \delta 8.57-8.33(\mathrm{~m}$, 1H), 7.75-7.31 (m, 7H), $7.19(\mathrm{~d}, J=7.3 \mathrm{~Hz}, 1 \mathrm{H}), 5.78(\mathrm{~s}, 2 \mathrm{H}), 4.03(\mathrm{q}, J=7.3 \mathrm{~Hz}, 1 \mathrm{H}), 0.79(\mathrm{~d}, J=$ $7.3 \mathrm{~Hz}, 3 \mathrm{H}) ;{ }^{13} \mathrm{C} \mathrm{NMR}\left(100 \mathrm{MHz}, \mathrm{CDCl}_{3}\right) \delta(\mathrm{ppm}) 152.4,146.3,144.3,139.4,137.3,136.0,135.5$, $134.5,130.4,129.0,128.8,128.6,127.8,124.1$, 123.2, 115.7, 90.5, 42.0, 17.4; HRMS (ESI-TOF) $\mathrm{m} / \mathrm{z}:[\mathrm{M}+\mathrm{Na}]^{+}$Calcd for $\left[\mathrm{C}_{21} \mathrm{H}_{15} \mathrm{~N}_{3} \mathrm{O}_{2}+\mathrm{Na}\right]^{+}$364.1056; Found 364.1053.

In order to improve the enantioselectivity, several catalysts were screened, and catalyst $\mathbf{C 6}$ gave slightly better data, as summarized below:
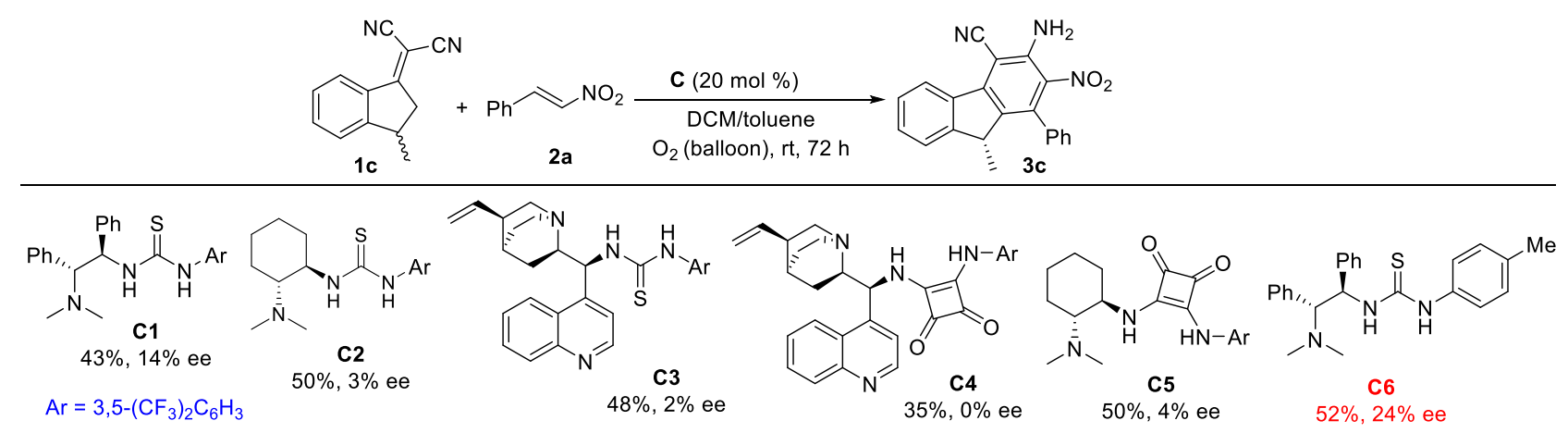


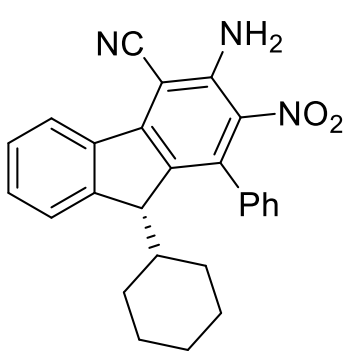

(R)-3-Amino-9-cyclohexyl-2-nitro-1-phenyl-9H-fluorene-4-carbonitrile

(3d): 2-(3-Cyclohexyl-2,3-dihydro-1 $H$-inden-1-ylidene)malononitrile $\quad$ 1d (26.2 mg, $0.0999 \mathrm{mmol}), \beta$-nitrostyrene 2a (29.8 mg, $0.200 \mathrm{mmol}, 2.0$ equiv) and $\mathbf{C 2}(8.3 \mathrm{mg}, 0.020 \mathrm{mmol}, 20 \mathrm{~mol} \%$ ) were added to a reaction tube, and the mixture was degassed and backfilled with oxygen (balloon). Dry toluene $(1.0 \mathrm{~mL})$ was added via syringe, and the mixture was stirred at $\mathrm{rt}$ for $10 \mathrm{~h}$. After the consumption of compound 1d, dry DCM (1.0 mL) was added via syringe, and the mixture was stirred for another 38 h. After completion (monitored by TLC), the solvent was evaporated in vacuo. The residue was purified by flash chromatography on silica gel $(\mathrm{PE} / \mathrm{EtOAc}=20: 1)$ to give the product 3d: $30.7 \mathrm{mg}$ $(0.0750 \mathrm{mmol})$, as a pale yellow solid, $75 \%$ yield; m.p. $206-208{ }^{\circ} \mathrm{C} ;[\alpha]_{\mathrm{D}}{ }^{25}=-306.0(c=0.029$ in $\mathrm{MeOH}$ ), 93\% ee, determined by HPLC analysis [Chiralpak IB, $n$-hexane $/ i-\mathrm{PrOH}=80 / 20,1.0 \mathrm{~mL} / \mathrm{min}$, $\lambda=254 \mathrm{~nm}, \mathrm{t}($ minor $)=6.47 \mathrm{~min}, \mathrm{t}$ (major) $=6.97 \mathrm{~min}] ;{ }^{1} \mathrm{H} \mathrm{NMR}\left(600 \mathrm{MHz}, \mathrm{CDCl}_{3}\right) \delta(\mathrm{ppm}) 8.48$ $(\mathrm{d}, J=7.7 \mathrm{~Hz}, 1 \mathrm{H}), 7.56-7.42(\mathrm{~m}, 7 \mathrm{H}), 7.18(\mathrm{~d}, J=6.7 \mathrm{~Hz}, 1 \mathrm{H}), 5.79(\mathrm{brs}, 2 \mathrm{H}), 4.06(\mathrm{~d}, J=2.9 \mathrm{~Hz}$, $1 \mathrm{H}), 1.60(\mathrm{~d}, J=10.5 \mathrm{~Hz}, 1 \mathrm{H}), 1.52(\mathrm{~d}, J=11.9 \mathrm{~Hz}, 1 \mathrm{H}), 1.46-1.44(\mathrm{~m}, 2 \mathrm{H}), 1.35(\mathrm{~d}, J=12.6 \mathrm{~Hz}$, 1H), 1.17-1.10 (m, 1H), 0.86-0.66 (m, 3H), $0.56(\mathrm{~d}, J=12.6 \mathrm{~Hz}, 1 \mathrm{H}), 0.08-0.02(\mathrm{~m}, 1 \mathrm{H}) ;{ }^{13} \mathrm{C}$ NMR $\left(100 \mathrm{MHz}, \mathrm{CDCl}_{3}\right) \delta(\mathrm{ppm}) 148.9,147.0,144.1,138.9,137.5,135.8,135.7,134.5,129.7,129.1$, $128.8,128.7,127.8,127.7,126.9,125.8,123.1,115.7,90.1,52.7,39.1,32.8,27.1,26.2,26.1,25.6$; HRMS (ESI-TOF) m/z: [M + Na $]^{+}$Calcd for $\left[\mathrm{C}_{26} \mathrm{H}_{23} \mathrm{~N}_{3} \mathrm{O}_{2}+\mathrm{Na}\right]^{+} 432.1683$; Found 432.1678.

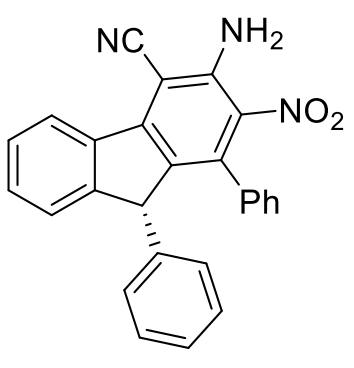

(R)-3-Amino-2-nitro-1,9-diphenyl-9H-fluorene-4-carbonitrile (3e): 2-(3Phenyl-2,3-dihydro-1H-inden-1-ylidene)malononitrile (1e) $(25.6$ mg, 0.0999 mmol), $\beta$-nitrostyrene $\mathbf{2 a}$ (29.8 mg, $0.200 \mathrm{mmol}, 2.0$ equiv) and $\mathbf{C 2}(8.3 \mathrm{mg}$, $0.020 \mathrm{mmol}, 20 \mathrm{~mol} \%$ ) were added to a reaction tube, and the mixture was degassed and backfilled with oxygen (balloon). Dry toluene $(1.0 \mathrm{~mL})$ was added by syringe, and the mixture was stirred at $\mathrm{rt}$ for $20 \mathrm{~h}$. After the consumption of compound 1e, dry DCM (1.0 mL) was added by syringe, and the mixture was stirred for another $38 \mathrm{~h}$. After completion (monitored by TLC), the solvent was evaporated in vacuo. The residue was purified by flash chromatography on silica gel $(\mathrm{PE} / \mathrm{EtOAc}=20: 1)$ to give the product 3e: $10.8 \mathrm{mg}(0.0268 \mathrm{mmol})$, as a yellow solid, $27 \%$ yield; m.p. $202-204{ }^{\circ} \mathrm{C} ; 27 \%$ ee, determined by HPLC analysis [Chiralpak IB, $n$-hexane $/ i-\mathrm{PrOH}=90 / 10,1.0 \mathrm{~mL} / \mathrm{min}, \lambda=254 \mathrm{~nm}, \mathrm{t}($ minor $)=11.51 \mathrm{~min}, \mathrm{t}$ (major) $=13.49 \mathrm{~min}]$; 
${ }^{1} \mathrm{H}$ NMR $\left(400 \mathrm{MHz}, \mathrm{CDCl}_{3}\right) \delta(\mathrm{ppm}) 8.48(\mathrm{~d}, J=7.8 \mathrm{~Hz}, 1 \mathrm{H}), 7.51-7.31(\mathrm{~m}, 4 \mathrm{H}), 7.25-7.14(\mathrm{~m}, 2 \mathrm{H})$, 7.02-6.81 (m, 4H), 6.49-6.22 (m, 3H), 5.85 (brs, 2H), $4.90(\mathrm{~s}, 1 \mathrm{H}) ;{ }^{13} \mathrm{C} \mathrm{NMR}\left(150 \mathrm{MHz}, \mathrm{CDCl}_{3}\right) \delta$ (ppm) 151.7, 147.0, 144.7, 140.4, 139.0, 137.3, 136.4, 135.0, 130.8, 128.20, 128.16, 128.10, 127.9, 127.8, 127.6, 127.2, 126.4, 125.5, 123.0, 115.6, 53.5; HRMS (ESI-TOF) m/z: [M - H] $]^{-}$Calcd for $\left[\mathrm{C}_{26} \mathrm{H}_{17} \mathrm{~N}_{3} \mathrm{O}_{2}-\mathrm{H}\right]^{-}$402.1248; Found 402.1247.

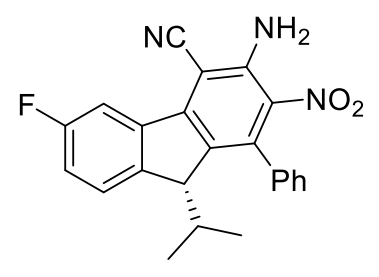

\section{(R)-3-Amino-6-fluoro-9-isopropyl-2-nitro-1-phenyl-9H-fluorene-4-}

carbonitrile (3f): 2-(6-Fluoro-3-isopropyl-2,3-dihydro-1 $H$-inden-1-ylidene) malononitrile 1f (24.0 mg, $0.100 \mathrm{mmol}), \beta$-nitrostyrene 2a $(29.8 \mathrm{mg}, 0.200$ mmol, 2.0 equiv) and $\mathbf{C 2}(8.3 \mathrm{mg}, 0.020 \mathrm{mmol}, 20 \mathrm{~mol} \%)$ were added to a reaction tube, and the mixture was degassed and backfilled with oxygen (balloon). Dry toluene (1.0 $\mathrm{mL}$ ) was added via syringe, and the mixture was stirred at $\mathrm{rt}$ for $10 \mathrm{~h}$. After the consumption of compound 1f, dry DCM (1.0 mL) was added via syringe, and the mixture was stirred for another 38 h. After completion (monitored by TLC), the solvent was evaporated in vacuo. The residue was purified by flash chromatography on silica gel $(\mathrm{PE} / \mathrm{EtOAc}=20: 1)$ to give the product 3f: $29.4 \mathrm{mg}$ (0.0759 mmol), as a yellow solid, 76\% yield; m.p. $216-218^{\circ} \mathrm{C} ;[\alpha]_{\mathrm{D}}{ }^{25}=-365.1(c=0.024$ in $\mathrm{MeOH})$, $77 \%$ ee, determined by HPLC analysis [Chiralpak AD-H, $n$-hexane $/ i-\mathrm{PrOH}=90 / 10,1.0 \mathrm{~mL} / \mathrm{min}, \lambda$ $=254 \mathrm{~nm}, \mathrm{t}($ major $)=6.51 \mathrm{~min}, \mathrm{t}($ minor $)=7.42 \mathrm{~min}] ;{ }^{1} \mathrm{H} \mathrm{NMR}\left(400 \mathrm{MHz}, \mathrm{CDCl}_{3}\right) \delta(\mathrm{ppm}) 8.15(\mathrm{dd}$, $J=9.0,2.4 \mathrm{~Hz}, 1 \mathrm{H}), 7.54-7.40(\mathrm{~m}, 5 \mathrm{H}), 7.17-7.12(\mathrm{~m}, 2 \mathrm{H}), 5.70$ (brs, 2H), 4.07 (d, $J=3.2 \mathrm{~Hz}, 1 \mathrm{H})$, $1.56-1.50(\mathrm{~m}, 1 \mathrm{H}), 0.95(\mathrm{~d}, J=7.0 \mathrm{~Hz}, 3 \mathrm{H}),-0.05(\mathrm{~d}, J=6.8 \mathrm{~Hz}, 3 \mathrm{H}) ;{ }^{13} \mathrm{C} \mathrm{NMR}\left(100 \mathrm{MHz}, \mathrm{CDCl}_{3}\right)$ $\delta(\mathrm{ppm}) 162.6\left(\mathrm{~d},{ }^{1} J_{\mathrm{FC}}=245.0 \mathrm{~Hz}\right), 145.6\left(\mathrm{~d},{ }^{3} J_{\mathrm{FC}}=3.0 \mathrm{~Hz}\right), 143.9,143.5\left(\mathrm{~d},{ }^{3} J_{\mathrm{FC}}=3.0 \mathrm{~Hz}\right), 139.4$, $138.9,136.8,135.3,135.2,129.5,129.2,129.1,128.9,127.8,126.8,126.7,116.7\left(\mathrm{~d},{ }^{2} J_{\mathrm{FC}}=23.0 \mathrm{~Hz}\right)$, $115.3,110.1,109.8\left(\mathrm{~d},{ }^{2} J_{\mathrm{FC}}=24.0 \mathrm{~Hz}\right), 90.4,52.3,28.4,22.0,14.7 ;{ }^{19} \mathrm{~F} \mathrm{NMR}\left(376 \mathrm{MHz}, \mathrm{CDCl}_{3}\right): \delta$ (ppm) -113.2; HRMS (ESI-TOF) m/z: $[\mathrm{M}+\mathrm{Na}]^{+} \mathrm{Calcd}$ for $\left[\mathrm{C}_{23} \mathrm{H}_{18} \mathrm{FN}_{3} \mathrm{O}_{2}+\mathrm{Na}\right]^{+}$410.1276; Found 410.1278 .

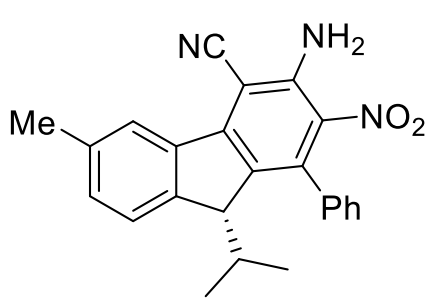

(R)-3-Amino-9-isopropyl-6-methyl-2-nitro-1-phenyl-9H-fluorene-4carbonitrile (3g): 2-(3-Isopropyl-6-methyl-2,3-dihydro-1H-inden-1ylidene)malononitrile $\mathbf{1 g}(23.6 \mathrm{mg}, 0.0999 \mathrm{mmol}), \beta$-nitrostyrene $2 \mathbf{a}$ (29.8 mg, $0.200 \mathrm{mmol}, 2.0$ equiv) and $\mathbf{C 2}$ ( $8.3 \mathrm{mg}, 0.020 \mathrm{mmol}, 20 \mathrm{~mol} \%)$ 
were added to a reaction tube, and the mixture was degassed and backfilled with oxygen (balloon). Dry toluene $(1.0 \mathrm{~mL})$ was added via syringe, and the mixture was stirred at $\mathrm{rt}$ for $10 \mathrm{~h}$. After the consumption of compound $\mathbf{1 g}$, dry DCM $(1.0 \mathrm{~mL})$ was added via syringe, and the mixture was stirred for another $38 \mathrm{~h}$. After completion (monitored by TLC), the solvent was evaporated in vacuo. The residue was purified by flash chromatography on silica gel $(\mathrm{PE} / \mathrm{EtOAc}=20: 1)$ to give the product 3g: $25.7 \mathrm{mg}(0.0670 \mathrm{mmol})$, as a yellow solid, 67\% yield; m.p. $248-250{ }^{\circ} \mathrm{C} ;[\alpha]_{\mathrm{D}}{ }^{25}=-166.9(c=0.032$ in $\mathrm{MeOH}$ ), $85 \%$ ee, determined by HPLC analysis [Chiralpak IC, $n$-hexane $/ i$-PrOH = 90/10, 1.0 $\mathrm{mL} / \mathrm{min}, \lambda=254 \mathrm{~nm}, \mathrm{t}$ (minor) $=16.47 \mathrm{~min}, \mathrm{t}$ (major) $=17.56 \mathrm{~min}] ;{ }^{1} \mathrm{H} \mathrm{NMR}\left(400 \mathrm{MHz}, \mathrm{CDCl}_{3}\right) \delta$ (ppm) 8.32 (s, 1H), 7.58-7.45 (m, 5H), 7.31-7.29 (m, 1H), 7.21-7.19 (d, J=8.4 Hz, 1H), 5.80 (brs, $2 \mathrm{H}), 4.10(\mathrm{~d}, J=3.2 \mathrm{~Hz}, 1 \mathrm{H}), 2.4(\mathrm{~s}, 3 \mathrm{H}), 1.61-1.52(\mathrm{~m}, 1 \mathrm{H}), 1.00(\mathrm{~d}, J=7.0 \mathrm{~Hz}, 3 \mathrm{H}),-0.01(\mathrm{~d}, J=$ $6.8 \mathrm{~Hz}, 3 \mathrm{H}) ;{ }^{13} \mathrm{C} \mathrm{NMR}\left(100 \mathrm{MHz}, \mathrm{CDCl}_{3}\right) \delta(\mathrm{ppm}) 147.0,145.4,144.1,138.8,137.9,137.8,136.4$, 135.7, 134.5, 130.8, 129.0, 128.7, 127.8, 126.8, 125.5, 123.4, 115.8, 90.0, 52.3, 28.4, 22.0, 21.6, 14.7; HRMS (ESI-TOF) m/z: [M + Na $]^{+}$Calcd for $\left[\mathrm{C}_{24} \mathrm{H}_{21} \mathrm{~N}_{3} \mathrm{O}_{2}+\mathrm{Na}\right]^{+} 406.1526$; Found 406.1520.

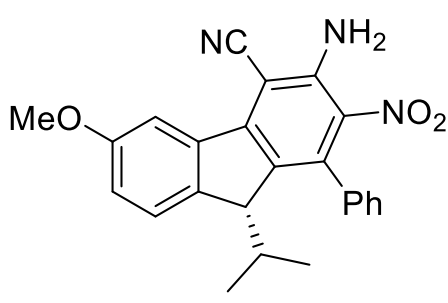

(R)-3-Amino-9-isopropyl-6-methoxy-2-nitro-1-phenyl-9H-fluorene-

4-carbonitrile (3h): 2-(3-Isopropyl-6-methoxyl-2,3-dihydro- $1 H$-inden1-ylidene)malononitrile $\mathbf{1 h}(25.2 \mathrm{mg}, 0.0999 \mathrm{mmol}), \beta$-nitrostyrene $\mathbf{2 a}$ (29.8 mg, $0.200 \mathrm{mmol}, 2.0$ equiv) and C2 (8.3 mg, $0.020 \mathrm{mmol}, 20$ mol \%) were added to a tube, and the mixture was degassed and backfilled with oxygen (balloon). Dry toluene $(1.0 \mathrm{~mL})$ was added via syringe, and the mixture was stirred at $\mathrm{rt}$ for $10 \mathrm{~h}$. After the consumption of compound $\mathbf{1 h}$, dry DCM $(1.0 \mathrm{~mL})$ was added via syringe, and the mixture was stirred for another $38 \mathrm{~h}$. After completion (monitored by TLC), the solvent was evaporated in vacuo. The residue was purified by flash chromatography on silica gel $(\mathrm{PE} / \mathrm{EtOAc}=20: 1)$ to give the product $\mathbf{3 h}$ : $26.3 \mathrm{mg}(0.0658 \mathrm{mmol})$ as a pale yellow solid, $66 \%$ yield; m.p. $198-200{ }^{\circ} \mathrm{C} ;[\alpha]_{\mathrm{D}}{ }^{25}=-200(c=0.02$ in $\mathrm{MeOH}$ ), $86 \%$ ee, determined by HPLC analysis [Chiralpak IB, $n$-hexane $/ i$-PrOH = 90/10, 1.0 $\mathrm{mL} / \mathrm{min}, \lambda=254 \mathrm{~nm}, \mathrm{t}$ (minor) $=10.58 \mathrm{~min}, \mathrm{t}$ (major) $=11.40 \mathrm{~min}] ;{ }^{1} \mathrm{H} \mathrm{NMR}\left(400 \mathrm{MHz}, \mathrm{CDCl}_{3}\right) \delta$ (ppm) $8.05(\mathrm{~d}, J=2.4 \mathrm{~Hz}, 1 \mathrm{H}), 7.57-7.45(\mathrm{~m}, 5 \mathrm{H}), 7.20(\mathrm{~d}, J=7.3 \mathrm{~Hz}, 1 \mathrm{H}), 7.05(\mathrm{dd}, J=8.4,2.5$ Hz, 1H), 5.78 (brs, 2H), 4.08 (d, J=3.2 Hz, 1H), 3.98 (s, 3H), 1.59-1.52 (m, 1H), 0.99 (d, J = 7.0 $\mathrm{Hz}, 3 \mathrm{H}),-0.01(\mathrm{~d}, J=6.8 \mathrm{~Hz}, 3 \mathrm{H}) ;{ }^{13} \mathrm{C} \mathrm{NMR}\left(100 \mathrm{MHz}, \mathrm{CDCl}_{3}\right) \delta(\mathrm{ppm}) 159.6,146.9,144.0,140.5$, 138.9, 138.7, 136.9, 135.6, 134.6, 129.0, 128.7, 127.8, 126.8, 126.4, 117.2, 115.8, 106.7, 90.0, 55.6, 
52.1, 28.4, 22.0, 14.7; HRMS (ESI-TOF) m/z: $[\mathrm{M}+\mathrm{Na}]^{+}$Calcd for $\left[\mathrm{C}_{24} \mathrm{H}_{21} \mathrm{~N}_{3} \mathrm{O}_{3}+\mathrm{Na}\right]^{+} 422.1476$; Found 422.1480 .<smiles>Cc1ccc2c(c1)[C@H](C(C)C)c1c-2c(C#N)c(N)c([N+](=O)[O-])c1-c1ccccc1</smiles>

(R)-3-Amino-9-isopropyl-7-methyl-2-nitro-1-phenyl-9H-fluorene-

4-carbonitrile (3i): 2-(3-Isopropyl-5-methyl-2,3-dihydro-1H-inden-1ylidene)malononitrile $\mathbf{1 i}$ (23.6 mg, $0.0999 \mathrm{mmol}), \beta$-nitrostyrene 2a (29.8 mg, 0.200 mmol, 2.0 equiv) and $\mathbf{C 2}(8.3 \mathrm{mg}, 0.020 \mathrm{mmol}, 20 \mathrm{~mol} \%)$ Dry toluene $(1.0 \mathrm{~mL})$ was added via syringe, and the mixture was stirred at $\mathrm{rt}$ for $10 \mathrm{~h}$. After the consumption of compound 1i, dry DCM $(1.0 \mathrm{~mL})$ was added via syringe, and the mixture was stirred for another $38 \mathrm{~h}$. After completion (monitored by TLC), the solvent was evaporated in vacuo. The residue was purified by flash chromatography on silica gel $(\mathrm{PE} / \mathrm{EtOAc}=20: 1)$ to give the product 3i: $22.2 \mathrm{mg}(0.0579 \mathrm{mmol})$, as a pale yellow solid, 58\% yield; m.p. $251-253{ }^{\circ} \mathrm{C} ;[\alpha]_{\mathrm{D}}{ }^{25}=-393.3(c=$ 0.06 in $\mathrm{MeOH}$ ), $85 \%$ ee, determined by HPLC analysis [Chiralpak IG, $n$-hexane/ $i$-PrOH = 90/10, 1.0 $\mathrm{mL} / \mathrm{min}, \lambda=254 \mathrm{~nm}, \mathrm{t}$ (major) $=7.82 \mathrm{~min}, \mathrm{t}$ (minor) $=8.75 \mathrm{~min}] ;{ }^{1} \mathrm{H} \mathrm{NMR}\left(600 \mathrm{MHz}, \mathrm{CDCl}_{3}\right) \delta$ (ppm) $8.33(\mathrm{~d}, J=8.0 \mathrm{~Hz}, 1 \mathrm{H}), 7.52-7.48(\mathrm{~m}, 2 \mathrm{H}), 7.46-7.40(\mathrm{~m}, 2 \mathrm{H}), 7.32(\mathrm{~s}, 1 \mathrm{H}), 7.29(\mathrm{~d}, J=8.0$ Hz, 1H), 7.15 (d, J=6.9 Hz, 1H), 5.76 (brs, 2H), 4.04 (d, J = 3.2 Hz, 1H), 2.46 (s, 3H), 1.54-1.51 $(\mathrm{m}, 1 \mathrm{H}), 0.95(\mathrm{~d}, J=7.0 \mathrm{~Hz}, 3 \mathrm{H}),-0.05(\mathrm{~d}, J=6.8 \mathrm{~Hz}, 3 \mathrm{H}) ;{ }^{13} \mathrm{C} \mathrm{NMR}\left(100 \mathrm{MHz}, \mathrm{CDCl}_{3}\right) \delta(\mathrm{ppm})$ 148.6, 147.3, 144.2, 140.3, 138.8, 135.9, 135.8, 135.2, 134.1, 129.0, 128.8, 128.6, 127.8, 126.8, 126.5, 122.8, 115.8, 89.7, 52.5, 28.5, 22.1, 21.9, 14.8; HRMS (ESI-TOF) m/z: $[\mathrm{M}+\mathrm{Na}]^{+}$: Calcd for $\left[\mathrm{C}_{24} \mathrm{H}_{21} \mathrm{~N}_{3} \mathrm{O}_{2}+\mathrm{Na}\right]^{+}$406.1526; Found 406.1524.

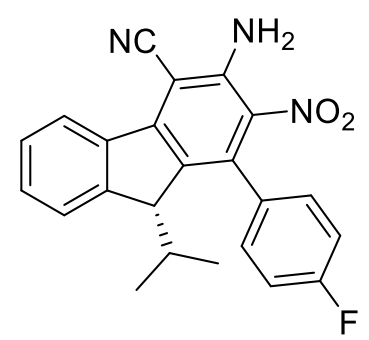

(R)-3-Amino-1-(4-fluorophenyl)-9-isopropyl-2-nitro-9H-fluorene-4carbonitrile (3j): 2-(3-Isopropyl-2,3-dihydro-1H-inden-1-ylidene)malononitrile $1 \mathbf{a}(22.2 \mathrm{mg}, 0.0999 \mathrm{mmol}),(E)$-1-fluoro-4-(2-nitrovinyl)benzene $\mathbf{2 b}$ (33.4 mg, $0.200 \mathrm{mmol}, 2.0$ equiv) and $\mathbf{C 2}(8.3 \mathrm{mg}, 0.020 \mathrm{mmol}, 20 \mathrm{~mol} \%$ ) were added to a reaction tube, and the mixture was degassed and backfilled with oxygen (balloon). Dry toluene $(1.0 \mathrm{~mL})$ was added via syringe, and the mixture was stirred at $\mathrm{rt}$ for $10 \mathrm{~h}$. After the consumption of compound 1a, dry DCM $(1.0 \mathrm{~mL})$ was added via syringe, and the mixture was stirred for another $38 \mathrm{~h}$. After completion (monitored by TLC), the solvent was 
evaporated in vacuo. The residue was purified by flash chromatography on silica gel (PE/EtOAc $=$ 20:1) to give the product $\mathbf{3 j}$ : $25.9 \mathrm{mg}(0.0668 \mathrm{mmol})$ as a pale yellow solid, 67\% yield; m.p. 196-198 ${ }^{\circ} \mathrm{C} ;[\alpha]_{\mathrm{D}}^{25}=-307.8(c=0.012$ in $\mathrm{MeOH}), 92 \%$ ee, determined by HPLC analysis [Chiralpak AD-H, $n$-hexane $/ \mathrm{i}-\mathrm{PrOH}=80 / 20,1.0 \mathrm{~mL} / \mathrm{min}, \lambda=254 \mathrm{~nm}, \mathrm{t}$ (minor) $=4.64 \mathrm{~min}, \mathrm{t}$ (major) $=5.11 \mathrm{~min}] ;{ }^{1} \mathrm{H}$ NMR (400 MHz, $\left.\mathrm{CDCl}_{3}\right) \delta(\mathrm{ppm}) 8.40(\mathrm{~d}, J=7.3 \mathrm{~Hz}, 1 \mathrm{H}), 7.50-7.34(\mathrm{~m}, 4 \mathrm{H}), 7.16(\mathrm{~d}, J=8.3 \mathrm{~Hz}$, 1H), 7.08-7.03 (d, $J=6.5 \mathrm{~Hz}, 2 \mathrm{H}), 5.70$ (brs, 2H), 3.98 (d, J=3.3 Hz, 1H), 1.55-1.45 (m, 1H), 0.93 $(\mathrm{d}, J=7.0 \mathrm{~Hz}, 3 \mathrm{H}),-0.12(\mathrm{~d}, J=6.8 \mathrm{~Hz}, 3 \mathrm{H}) ;{ }^{13} \mathrm{C} \mathrm{NMR}\left(100 \mathrm{MHz}, \mathrm{CDCl}_{3}\right) \delta(\mathrm{ppm}) 161.8\left({ }^{1} J_{\mathrm{FC}}=\right.$ $247.5 \mathrm{~Hz}), 147.0,146.1,143.1,136.7,136.6,135.0,133.4,130.5,130.5,128.8,128.6\left({ }^{3} J_{\mathrm{FC}}=7.4 \mathrm{~Hz}\right)$, $127.7\left({ }^{3} J_{\mathrm{FC}}=7.4 \mathrm{~Hz}\right), 126.9,124.8,122.1,115.3\left({ }^{2} J_{\mathrm{FC}}=22.2 \mathrm{~Hz}\right), 115.2\left({ }^{2} J_{\mathrm{FC}}=21.8 \mathrm{~Hz}\right), 114.6,89.3$, 51.6, 27.6, 21.0, 13.8; ${ }^{19} \mathrm{~F}$ NMR (376 $\left.\mathrm{MHz}, \mathrm{CDCl}_{3}\right) \delta(\mathrm{ppm})-112.1$; HRMS (ESI-TOF) m/z: [M + $\mathrm{Na}]^{+}$Calcd for $\left[\mathrm{C}_{23} \mathrm{H}_{18} \mathrm{FN}_{3} \mathrm{O}_{2}+\mathrm{Na}\right]^{+}$410.1276; Found 410.1270.

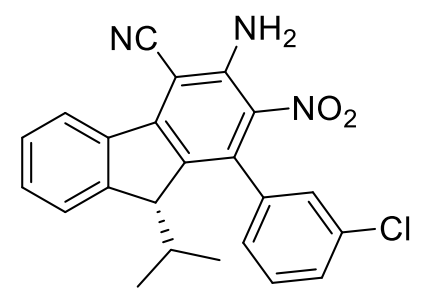

\section{(R)-3-Amino-1-(3-chlorophenyl)-9-isopropyl-2-nitro-9H-fluorene-4-} carbonitrile (3k): 2-(3-Isopropyl-2,3-dihydro-1H-inden-1-ylidene)malononitrile 1a (22.2 mg, $0.0999 \mathrm{mmol}),(E)$-1-chloro-3-(2-nitrovinyl)benzene

2c (36.6 mg, $0.199 \mathrm{mmol}, 2.0$ equiv) and $\mathbf{C 2}$ (8.3 mg, $0.020 \mathrm{mmol}, 20$ mol \%) were added to a reaction tube, and the mixture was degassed and backfilled with oxygen (balloon). Dry toluene $(1.0 \mathrm{~mL})$ was added via syringe, the mixture was stirred at $\mathrm{rt}$ for $10 \mathrm{~h}$. After the consumption of compound 1a, dry DCM $(1.0 \mathrm{~mL})$ was added via syringe, and the mixture was stirred for another $38 \mathrm{~h}$. After completion (monitored by TLC), the solvent was evaporated in vacuo. The residue was purified by flash chromatography on silica gel $(\mathrm{PE} / \mathrm{EtOAc}=20: 1)$ to give the product 3k: $30.2 \mathrm{mg}(0.0748 \mathrm{mmol})$ as a pale yellow solid, 75\% yield; m.p. $224-226^{\circ} \mathrm{C} ;[\alpha]_{\mathrm{D}}{ }^{25}=-217.8(c$ $=0.014$ in $\mathrm{MeOH}$ ), $84 \%$ ee, determined by HPLC analysis [Chiralpak AD-H, $n$-hexane $/ i$-PrOH $=$ $80 / 20,1.0 \mathrm{~mL} / \mathrm{min}, \lambda=254 \mathrm{~nm}, \mathrm{t}$ (major) $=4.64 \mathrm{~min}, \mathrm{t}$ (minor) $=5.12 \mathrm{~min}] ;{ }^{1} \mathrm{H}$ NMR $(400 \mathrm{MHz}$, DMSO- $\left.d_{6}\right) \delta(\mathrm{ppm}) 8.37(\mathrm{~d}, J=7.6 \mathrm{~Hz}, 1 \mathrm{H}), 7.83-7.17(\mathrm{~m}, 7 \mathrm{H}), 6.71$ (brs, 2H), 4.20-4.04 (m, 1H), $1.57-1.39(\mathrm{~m}, 1 \mathrm{H}), 0.94(\mathrm{~d}, J=7.0 \mathrm{~Hz}, 3 \mathrm{H}),-0.06--0.22(\mathrm{~m}, 3 \mathrm{H}) ;{ }^{13} \mathrm{C}$ NMR $\left(100 \mathrm{MHz}, \mathrm{DMSO}-d_{6}\right)$ $\delta(\mathrm{ppm}) 147.6 ., 147.5,145.3,137.3,136.8,135.4,130.8,130.6,129.3,128.7,127.8,127.7,126.3$, 125.8, 125.5, 121.8, 115.4, 89.1, 51.4, 39.8, 27.9, 21.5, 14.7; HRMS (ESI-TOF) m/z: $[\mathrm{M}+\mathrm{Na}]^{+} \mathrm{Calcd}$ for $\left[\mathrm{C}_{23} \mathrm{H}_{18}{ }^{35} \mathrm{ClN}_{3} \mathrm{O}_{2}+\mathrm{Na}\right]^{+}$426.0980; Found 426.0972; Calcd for $\left[\mathrm{C}_{23} \mathrm{H}_{18}{ }^{37} \mathrm{ClN}_{3} \mathrm{O}_{2}+\mathrm{Na}^{+} 428.0951\right.$; Found 428.0954. 


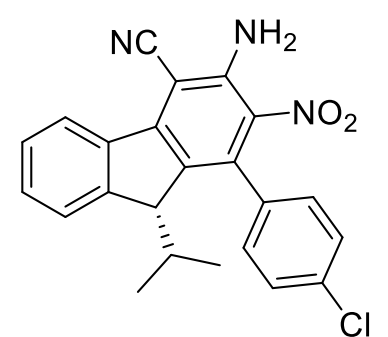

(R)-3-Amino-1-(4-chlorophenyl)-9-isopropyl-2-nitro-9H-fluorene-4-

carbonitrile (31): 2-(3-Isopropyl-2,3-dihydro-1H-inden-1-ylidene)malononitrile 1a (22.2 mg, $0.0999 \mathrm{mmol}),(E)$-1-chloro-4-(2-nitrovinyl)benzene 2d (36.6 mg, 0.199 mmol, 2.0 equiv) and $\mathbf{C 2}$ (8.3 mg, $0.020 \mathrm{mmol}, 20 \mathrm{~mol} \%)$ were added to a reaction tube, and the mixture was degassed and backfilled with oxygen (balloon). Dry toluene $(1.0 \mathrm{~mL})$ was added by syringe, and the mixture was stirred at $\mathrm{rt}$ for $10 \mathrm{~h}$. After the consumption of compound 1a, dry DCM $(1.0 \mathrm{~mL})$ was added by syringe, and the mixture was stirred for another $38 \mathrm{~h}$. After completion (monitored by TLC), the solvent was evaporated in vacuo. The residue was purified by flash chromatography on silica gel $(\mathrm{PE} / \mathrm{EtOAc}=$ 20:1) to give the product 3l: $25.4 \mathrm{mg}(0.0629 \mathrm{mmol})$, as a pale yellow solid, 63\% yield; m.p. 234-236 ${ }^{\circ} \mathrm{C} ;[\alpha]_{\mathrm{D}}^{25}=-93.8(c=0.057$ in $\mathrm{MeOH}), 90 \%$ ee, determined by HPLC analysis [Chiralpak AD-H, $n$-hexane $/ i-\mathrm{PrOH}=80 / 20,1.0 \mathrm{~mL} / \mathrm{min}, \lambda=254 \mathrm{~nm}, \mathrm{t}$ (minor) $=4.69 \mathrm{~min}, \mathrm{t}$ (major) $=5.35 \mathrm{~min}] ;{ }^{1} \mathrm{H}$ NMR $\left(400 \mathrm{MHz}, \mathrm{CDCl}_{3}\right) \delta(\mathrm{ppm}) 8.47(\mathrm{~d}, J=7.6 \mathrm{~Hz}, 1 \mathrm{H}), 7.55-7.41(\mathrm{~m}, 6 \mathrm{H}), 7.11(\mathrm{~d}, J=7.6 \mathrm{~Hz}$, 1H), 5.81 (brs, 2H), $4.06(\mathrm{~d}, J=3.1 \mathrm{~Hz}, 1 \mathrm{H}), 1.61-1.58(\mathrm{~m}, 1 \mathrm{H}), 1.01(\mathrm{~d}, J=7.0 \mathrm{~Hz}, 3 \mathrm{H}),-0.05(\mathrm{~d}$, $J=6.8 \mathrm{~Hz}, 3 \mathrm{H}) ;{ }^{13} \mathrm{C} \mathrm{NMR}\left(100 \mathrm{MHz}, \mathrm{CDCl}_{3}\right) \delta(\mathrm{ppm}) 148.0,147.2,144.2,137.6,135.8,134.8,134.2$, 134.1, 129.8, 129.39, 129.35, 129.1, 128.2, 128.0, 125.8, 123.1, 115.5, 90.4, 52.6, 28.7, 22.0, 14.8; HRMS (ESI-TOF) m/z: $[\mathrm{M}+\mathrm{Na}]^{+}$Calcd for $\left[\mathrm{C}_{23} \mathrm{H}_{18}{ }^{35} \mathrm{ClN}_{3} \mathrm{O}_{2}+\mathrm{Na}\right]^{+}$426.0980; Found 426.0981; Calcd for $\left[\mathrm{C}_{23} \mathrm{H}_{18}{ }^{37} \mathrm{ClN}_{3} \mathrm{O}_{2}+\mathrm{Na}\right]^{+} 428.0951$; Found 428.0963.

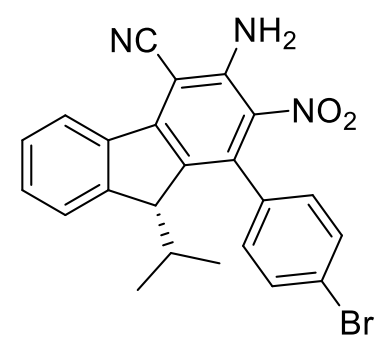

(R)-3-Amino-1-(4-bromophenyl)-9-isopropyl-2-nitro-9H-fluorene-4carbonitrile (3m): 2-(3-Isopropyl-2,3-dihydro-1H-inden-1-ylidene)malononitrile $1 \mathbf{a}(22.2 \mathrm{mg}, 0.0999 \mathrm{mmol}),(E)$-1-bromo-4-(2-nitrovinyl)benzene 2e (45.6 mg, $0.200 \mathrm{mmol}, 2.0$ equiv) and $\mathbf{C 2}(8.3 \mathrm{mg}, 0.020 \mathrm{mmol}, 20 \mathrm{~mol} \%)$ were added to a reaction tube, and the mixture was degassed and backfilled with oxygen (balloon). Dry toluene $(1.0 \mathrm{~mL})$ was added via syringe, and the mixture was stirred at $\mathrm{rt}$ for $10 \mathrm{~h}$. After the consumption of compound 1a, dry DCM (1.0 mL) was added via syringe, and the mixture was stirred for another $38 \mathrm{~h}$. After completion (monitored by TLC), the solvent was evaporated in vacuo. The residue was purified by flash chromatography on silica gel (PE/EtOAc $=$ 20:1) to give the product 3m: $27.7 \mathrm{mg}(0.0618 \mathrm{mmol})$, as a yellow solid, 62\% yield; m.p. 173-175 ${ }^{\circ} \mathrm{C} ;[\alpha]_{\mathrm{D}}{ }^{25}=-129.3(c=0.076$ in $\mathrm{MeOH}), 89 \%$ ee, determined by HPLC analysis [Chiralpak AD-H, 
$n$-hexane $/ i-\mathrm{PrOH}=80 / 20,1.0 \mathrm{~mL} / \mathrm{min}, \lambda=254 \mathrm{~nm}, \mathrm{t}$ (minor) $=4.75 \mathrm{~min}, \mathrm{t}$ (major) $=5.46 \mathrm{~min}] ;{ }^{1} \mathrm{H}$ NMR $\left(600 \mathrm{MHz}, \mathrm{CDCl}_{3}\right) \delta(\mathrm{ppm}) 8.47(\mathrm{~d}, J=7.7 \mathrm{~Hz}, 1 \mathrm{H}), 7.67(\mathrm{~d}, J=7.7 \mathrm{~Hz}, 1 \mathrm{H}), 7.57(\mathrm{~d}, J=7.7$ $\mathrm{Hz}, 1 \mathrm{H}), 7.54(\mathrm{~d}, J=7.5 \mathrm{~Hz}, 1 \mathrm{H}), 7.50(\mathrm{t}, J=7.3 \mathrm{~Hz}, 1 \mathrm{H}), 7.45(\mathrm{t}, J=7.2 \mathrm{~Hz}, 1 \mathrm{H}), 7.38(\mathrm{~d}, J=7.5$ Hz, 1H), 7.05 (d, J=7.5 Hz, 1H), 5.82 (brs, 2H), 4.06 (d, $J=3.2 \mathrm{~Hz}, 1 \mathrm{H}), 1.62-1.59$ (m, 1H), 1.01 $(\mathrm{d}, J=7.0 \mathrm{~Hz}, 3 \mathrm{H}),-0.04(\mathrm{~d}, J=6.8 \mathrm{~Hz}, 3 \mathrm{H}) ;{ }^{13} \mathrm{C} \mathrm{NMR}\left(150 \mathrm{MHz}, \mathrm{CDCl}_{3}\right) \delta(\mathrm{ppm}) 148.0,147.2$, 144.3, 137.58, 137.57, 135.7, 134.6, 134.1, 132.3, 129.8, 129.4, 128.5, 128.0, 125.8, 123.2, 123.0, 115.5, 90.5, 52.6, 28.7, 22.0, 14.8; HRMS (ESI-TOF) m/z: $[\mathrm{M}-\mathrm{H}]^{-}$Calcd for $\left[\mathrm{C}_{23} \mathrm{H}_{17}{ }^{79} \mathrm{BrN}_{3} \mathrm{O}_{2}\right.$ $-\mathrm{H}]^{-}$446.0509; Found 446.0502; $\left[\mathrm{C}_{23} \mathrm{H}_{17}{ }^{81} \mathrm{BrN}_{3} \mathrm{O}_{2}-\mathrm{H}\right]^{-} 448.0489$; Found 446.0475.

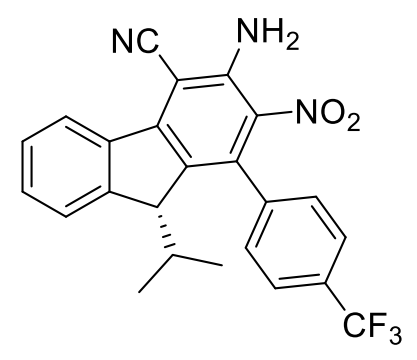

(R)-3-Amino-9-isopropyl-2-nitro-1-(4-(trifluoromethyl)phenyl)-9Hfluorene-4-carbonitrile (3n): 2-(3-Isopropyl-2,3-dihydro-1H-inden-1ylidene)malononitrile 1a (22.2 mg, $0.0999 \mathrm{mmol}),(E)-1-(2-n i t r o v i n y l)-4-$ (trifluoromethyl)benzene $\mathbf{2 f}$ (43.4 $\mathrm{mg}, 0.199 \mathrm{mmol}, 2.0$ equiv) and $\mathbf{C 2}$ (8.3 $\mathrm{mg}, 0.020 \mathrm{mmol}, 20 \mathrm{~mol} \%$ ) were added to a reaction tube, and the mixture was degassed and backfilled with oxygen (balloon). Dry toluene (1.0 mL) was added via syringe, and the mixture was stirred at $\mathrm{rt}$ for $10 \mathrm{~h}$. After the consumption of compound 1a, dry DCM (1.0 mL) was added via syringe, and the mixture was stirred for another $38 \mathrm{~h}$. After completion (monitored by TLC), the solvent was evaporated in vacuo. The residue was purified by flash chromatography on silica gel $(\mathrm{PE} / \mathrm{EtOAc}=20: 1)$ to give the product $3 \mathrm{n}: 24.5 \mathrm{mg}(0.0560 \mathrm{mmol})$, as a pale yellow solid, $56 \%$ yield; m.p. $226-228{ }^{\circ} \mathrm{C} ;[\alpha]_{\mathrm{D}}{ }^{25}=-268.1(c=0.148$ in $\mathrm{MeOH}), 83 \%$ ee, determined by HPLC analysis [Chiralpak AD-H, $n$-hexane $/ i-\mathrm{PrOH}=80 / 20,1.0 \mathrm{~mL} / \mathrm{min}, \lambda=254 \mathrm{~nm}, \mathrm{t}$ (minor) $=4.09$ min, $\mathrm{t}$ (major) $=4.62 \mathrm{~min}] ;{ }^{1} \mathrm{H} \mathrm{NMR}\left(400 \mathrm{MHz}, \mathrm{CDCl}_{3}\right) \delta(\mathrm{ppm}) 8.49(\mathrm{~d}, J=7.6 \mathrm{~Hz}, 1 \mathrm{H}), 7.81(\mathrm{~d}, J=7.8 \mathrm{~Hz}, 1 \mathrm{H})$, $7.70(\mathrm{~d}, J=7.7 \mathrm{~Hz}, 1 \mathrm{H}), 7.63(\mathrm{~d}, J=7.8 \mathrm{~Hz}, 1 \mathrm{H}), 7.55-7.44(\mathrm{~m}, 3 \mathrm{H}), 7.31(\mathrm{~d}, J=7.8 \mathrm{~Hz}, 1 \mathrm{H}), 5.93$ (brs, 2H), $4.04(\mathrm{~d}, J=3.1 \mathrm{~Hz}, 1 \mathrm{H}), 1.52-1.46(\mathrm{~m}, 1 \mathrm{H}), 0.97$ (d, $J=7.0 \mathrm{~Hz}, 3 \mathrm{H}),-0.04$ (d, J = 6.8 Hz, $3 \mathrm{H}) ;{ }^{13} \mathrm{C} \mathrm{NMR}\left(150 \mathrm{MHz}, \mathrm{CDCl}_{3}\right) \delta(\mathrm{ppm}) 148.0,147.5,144.5,139.6,137.4,4$ 137.42, 135.7, 133.7, $130.8\left(\mathrm{q},{ }^{2} J_{\mathrm{FC}}=33.0 \mathrm{~Hz}\right), 130.0,128.3,128.0,127.3,126.6,126.0-125.9(\mathrm{~m}), 125.8,123.9\left(\mathrm{q},{ }^{1} J_{\mathrm{FC}}\right.$ $=192 \mathrm{~Hz}), 123.2,115.4,90.7,52.5,28.8,21.9,14.7 ;{ }^{19} \mathrm{~F} \mathrm{NMR}\left(376 \mathrm{MHz}, \mathrm{CDCl}_{3}\right): \delta$ (ppm) -62.6; HRMS (ESI-TOF) m/z: $[\mathrm{M}-\mathrm{H}]^{-}$Calcd for $\left[\mathrm{C}_{24} \mathrm{H}_{17} \mathrm{~F}_{3} \mathrm{~N}_{3} \mathrm{O}_{2}-\mathrm{H}\right]^{-} 436.1278$; Found 436.1273. 


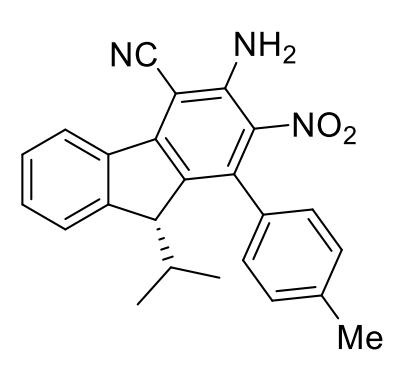

(R)-3-Amino-9-isopropyl-2-nitro-1-(p-tolyl)-9H-fluorene-4-carbonitrile

(3o): 2-(3-Isopropyl-2,3-dihydro-1H-inden-1-ylidene)malononitrile $\quad \mathbf{1 a}$ (22.2 mg, $0.0999 \mathrm{mmol}),(E)$-1-methyl-4-(2-nitrovinyl)benzene $2 \mathrm{~g}$ (32.6 mg, $0.200 \mathrm{mmol}, 2.0$ equiv) and $\mathbf{C 2}(8.3 \mathrm{mg}, 0.020 \mathrm{mmol}, 20 \mathrm{~mol} \%)$ were added

to a reaction tube, and the mixture was degassed and backfilled with oxygen (balloon). Dry toluene (1.0 mL) was added via syringe, and the mixture was stirred at $\mathrm{rt}$ for $10 \mathrm{~h}$. After the consumption of compound 1a, dry DCM $(1.0 \mathrm{~mL})$ was added via syringe, and the mixture was stirred for another $38 \mathrm{~h}$. After completion (monitored by TLC), the solvent was evaporated in vacuo. The residue was purified by flash chromatography on silica gel $(\mathrm{PE} / \mathrm{EtOAc}=20: 1)$ to give the product 3o: $23.4 \mathrm{mg}(0.0610 \mathrm{mmol})$, as a pale yellow solid, $61 \%$ yield; m.p. $224-226{ }^{\circ} \mathrm{C}$; $[\alpha]_{\mathrm{D}}{ }^{25}=$ $-163(c=0.06$ in $\mathrm{MeOH}), 86 \%$ ee, determined by HPLC analysis [Chiralpak IB, $n$-hexane $/ i$-PrOH = $80 / 20,1.0 \mathrm{~mL} / \mathrm{min}, \lambda=254 \mathrm{~nm}, \mathrm{t}$ (major) $=6.08 \mathrm{~min}, \mathrm{t}$ (minor) $=6.77 \mathrm{~min}] ;{ }^{1} \mathrm{H} \mathrm{NMR}(400 \mathrm{MHz}$, $\left.\mathrm{CDCl}_{3}\right) \delta(\mathrm{ppm}) 8.51(\mathrm{~d}, J=7.6 \mathrm{~Hz}, 1 \mathrm{H}), 7.59(\mathrm{~d}, J=7.4 \mathrm{~Hz}, 1 \mathrm{H}), 7.53(\mathrm{t}, J=7.2 \mathrm{~Hz}, 1 \mathrm{H}), 7.51-7.47$ (m, 1H), 7.47-7.34 (m, 2H), $7.28(\mathrm{~d}, J=5.8 \mathrm{~Hz}, 1 \mathrm{H}), 7.10(\mathrm{~d}, J=5.9 \mathrm{~Hz}, 1 \mathrm{H}), 5.73$ (brs, 2H), 4.17 $(\mathrm{d}, J=3.2 \mathrm{~Hz}, 1 \mathrm{H}), 2.49(\mathrm{~s}, 3 \mathrm{H}), 1.73-1.61(\mathrm{~m}, 1 \mathrm{H}), 1.04(\mathrm{~d}, J=7.0 \mathrm{~Hz}, 3 \mathrm{H}),-0.01(\mathrm{~d}, J=6.8 \mathrm{~Hz}$, $3 \mathrm{H}) ;{ }^{13} \mathrm{C}$ NMR $\left(100 \mathrm{MHz}, \mathrm{CDCl}_{3}\right) \delta$ (ppm) 147.1, 145.7, 142.9, 137.8, 137.7, 136.8, 135.0, 133.8, $131.4,128.8,128.5,126.8,126.6,125.6,124.7,122.0,114.8,88.9$, 51.7, 27.4, 21.0, 20.4, 13.7; HRMS (ESI-TOF) m/z: $[\mathrm{M}+\mathrm{Na}]^{+}$Calcd for $\left[\mathrm{C}_{24} \mathrm{H}_{21} \mathrm{~N}_{3} \mathrm{O}_{2}+\mathrm{Na}\right]^{+} 406.1526$; Found 406.1527.

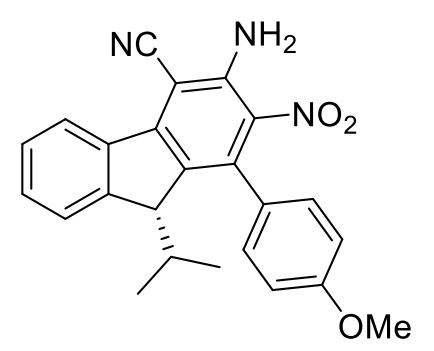

(R)-3-Amino-9-isopropyl-1-(4-methoxyphenyl)-2-nitro-9H-fluorene4-carbonitrile (3p): 2-(3-Isopropyl-2,3-dihydro-1H-inden-1-ylidene) malononitrile 1a (22.2 mg, $0.0999 \mathrm{mmol}),(E)-1$-methoxy-4-(2-nitrovinyl) benzene $2 \mathbf{h}$ (35.8 mg, $0.200 \mathrm{mmol}, 2.0$ equiv) and C2 (8.3 mg, $0.020 \mathrm{mmol}$, $20 \mathrm{~mol} \%$ ) were added to a reaction tube, and the mixture was degassed and backfilled with oxygen (balloon). Dry toluene $(1.0 \mathrm{~mL})$ was added via syringe, and the mixture was stirred at $\mathrm{rt}$ for $10 \mathrm{~h}$. After the consumption of compound 1a, dry DCM (1.0 mL) was added via syringe, and the mixture was stirred for another $38 \mathrm{~h}$. After completion (monitored by TLC), the solvent was evaporated in vacuo. The residue was purified by flash chromatography on silica gel $(\mathrm{PE} / \mathrm{EtOAc}=20: 1)$ to give the product 3p: $29.5 \mathrm{mg}(0.0738 \mathrm{mmol})$, as a yellow solid, $74 \%$ yield; m.p. $209-211^{\circ} \mathrm{C} ;[\alpha]_{\mathrm{D}}^{25}=-20.3(c=0.11$ in $\mathrm{MeOH}), 81 \%$ ee, determined by HPLC analysis [Chiralpak 
AD-H, $n$-hexane $/ i-\mathrm{PrOH}=80 / 20,1.0 \mathrm{~mL} / \mathrm{min}, \lambda=254 \mathrm{~nm}, \mathrm{t}$ (major) $=5.99 \mathrm{~min}, \mathrm{t}$ (minor) $=6.82$ min]; ${ }^{1} \mathrm{H}$ NMR $\left(400 \mathrm{MHz}, \mathrm{CDCl}_{3}\right) \delta(\mathrm{ppm}) 8.46(\mathrm{~d}, J=7.6 \mathrm{~Hz}, 1 \mathrm{H}), 7.54(\mathrm{~d}, J=7.4 \mathrm{~Hz}, 1 \mathrm{H}), 7.51-$ $7.33(\mathrm{~m}, 3 \mathrm{H}), 7.01-6.97(\mathrm{~m}, 3 \mathrm{H}), 5.64(\mathrm{brs}, 2 \mathrm{H}), 4.11(\mathrm{~d}, J=3.1 \mathrm{~Hz}, 1 \mathrm{H}), 3.88(\mathrm{~s}, 3 \mathrm{H}), 1.68-1.62(\mathrm{~m}$, 1H), $1.00(\mathrm{~d}, J=7.0 \mathrm{~Hz}, 3 \mathrm{H}),-0.06(\mathrm{~d}, J=6.8 \mathrm{~Hz}, 3 \mathrm{H}),{ }^{13} \mathrm{C} \mathrm{NMR}\left(100 \mathrm{MHz}, \mathrm{CDCl}_{3}\right) \delta(\mathrm{ppm}) 159.9$, 148.1, 146.6, 143.9, 138.5, 137.9, 136.2, 135.1, 129.5, 129.0, 128.1, 127.8, 127.5, 125.7, 123.0, 115.8, 114.8, 114.4, 89.8, 55.3, 52.7, 28.4, 22.0, 14.8; HRMS (ESI-TOF) m/z: $[\mathrm{M}+\mathrm{Na}]^{+}$Calcd for $\left[\mathrm{C}_{24} \mathrm{H}_{21} \mathrm{~N}_{3} \mathrm{O}_{3}+\mathrm{Na}\right]^{+}$422.1476; Found 422.1479.

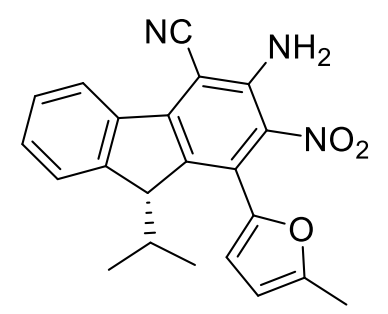

(R)-3-Amino-9-isopropyl-1-(5-methylfuran-2-yl)-2-nitro-9H-fluorene4-carbonitrile (3q): 2-(3-Isopropyl-2,3-dihydro-1H-inden-1-ylidene) malononitrile 1a (22.2 mg, $0.0999 \mathrm{mmol}),(E)$-2-methyl-5-(2-nitrovinyl)furan $\mathbf{2 i}$ (30.6 mg, $0.200 \mathrm{mmol}, 2.0$ equiv) and $\mathbf{C 2}$ (8.3 mg, $0.020 \mathrm{mmol}, 20 \mathrm{~mol} \%)$ were added to a reaction tube, and the mixture was degassed and backfilled with oxygen (balloon). Dry toluene $(1.0 \mathrm{~mL})$ was added via syringe, and the mixture was stirred at $\mathrm{rt}$ for $10 \mathrm{~h}$. After the consumption of compound 1a, dry DCM $(1.0 \mathrm{~mL})$ was added via syringe, and the mixture was stirred for another $38 \mathrm{~h}$. After completion (monitored by TLC), the solvent was evaporated in vacuo. The residue was purified by flash chromatography on silica gel $(\mathrm{PE} / \mathrm{EtOAc}=20: 1)$ to give the product 3q: $25.3 \mathrm{mg}(0.0678 \mathrm{mmol})$ as a yellow thick oil, $68 \%$ yield; $[\alpha]_{\mathrm{D}}{ }^{25}=-478.0\left(c=0.02\right.$ in $\left.\mathrm{CHCl}_{3}\right), 79 \%$ ee, determined by HPLC analysis [Chiralpak ID, $n$-hexane $/ i$-PrOH =90/10, $1.0 \mathrm{~mL} / \mathrm{min}, \lambda=254 \mathrm{~nm}$, $\mathrm{t}($ minor $)=8.79 \mathrm{~min}, \mathrm{t}($ major $)=15.89 \mathrm{~min}] ;{ }^{1} \mathrm{H} \mathrm{NMR}\left(400 \mathrm{MHz}, \mathrm{CDCl}_{3}\right) \delta(\mathrm{ppm}) 8.45-8.42(\mathrm{~m}$, 1H), 7.62-7.55 (m, 1H), 7.51-7.43 (m, 2H), 6.58 (d, J=3.3 Hz, 1H), 6.18-6.17 (m, 1H), 5.59 (brs, $2 \mathrm{H}), 4.36(\mathrm{~d}, J=2.8 \mathrm{~Hz}, 1 \mathrm{H}), 2.40(\mathrm{~s}, 3 \mathrm{H}), 1.92-1.85(\mathrm{~m}, 1 \mathrm{H}), 1.19(\mathrm{~d}, J=7.0 \mathrm{~Hz}, 3 \mathrm{H}),-0.01(\mathrm{~d}, J$ $=6.8 \mathrm{~Hz}, 3 \mathrm{H}) ;{ }^{13} \mathrm{C} \mathrm{NMR}\left(100 \mathrm{MHz}, \mathrm{CDCl}_{3}\right) \delta(\mathrm{ppm}) 154.3,148.1,146.9,144.6,143.7,137.6,135.6$, 133.1, 129.7, 127.8, 126.9, 125.5, 122.9, 115.7, 112.8, 108.3, 90.0, 53.1, 29.0, 22.2, 15.3, 13.6; HRMS (ESI-TOF) m/z: $[\mathrm{M}+\mathrm{Na}]^{+}$Calcd for $\left[\mathrm{C}_{22} \mathrm{H}_{19} \mathrm{~N}_{3} \mathrm{O}_{3}+\mathrm{Na}\right]^{+}$396.1319; Found 396.1318.

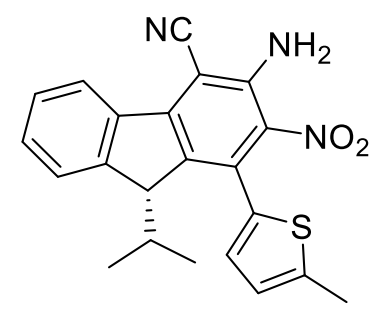

(R)-3-Amino-9-isopropyl-1-(5-methylthiophen-2-yl)-2-nitro-9H-fluorene-4-carbonitrile (3r): 2-(3-Isopropyl-2,3-dihydro-1H-inden-1-ylidene)malononitrile 1a (22.2 mg, $0.0999 \mathrm{mmol}),(E)$-2-methyl-5-(2-nitrovinyl)thiophene 2 j (33.8 mg, $0.200 \mathrm{mmol}, 2.0$ equiv) and C2 (8.3 mg, $0.020 \mathrm{mmol}, 20$ 
mol \%) were added to a reaction tube, and the mixture was degassed and backfilled with oxygen (balloon). Dry toluene (1.0 mL) was added via syringe, and the mixture was stirred at $\mathrm{rt}$ for $10 \mathrm{~h}$. After the consumption of compound 1a, dry DCM $(1.0 \mathrm{~mL})$ was added via syringe, and the mixture was stirred for another $38 \mathrm{~h}$. After completion (monitored by TLC), the solvent was evaporated in vacuo. The residue was purified by flash chromatography on silica gel (PE/EtOAc $=20: 1)$ to give the product 3r: $24.2 \mathrm{mg}(0.0621 \mathrm{mmol})$, as a yellow thick oil, $62 \%$ yield; $[\alpha]_{\mathrm{D}}{ }^{25}=-52(c=0.005$ in $\mathrm{CHCl}_{3}$, dark sample with low concentration), 62\% ee, determined by HPLC analysis [Chiralpak ID, $n$-hexane $/ i$-PrOH $=80 / 20,1.0 \mathrm{~mL} / \mathrm{min}, \lambda=254 \mathrm{~nm}, \mathrm{t}$ (major) $=6.55 \mathrm{~min}, \mathrm{t}($ minor $)=7.13 \mathrm{~min}] ;{ }^{1} \mathrm{H}$ NMR (400 MHz, $\left.\mathrm{CDCl}_{3}\right) \delta(\mathrm{ppm}) 8.45(\mathrm{~d}, J=7.3 \mathrm{~Hz}, 1 \mathrm{H}), 7.57$ (d, $\left.J=7.3 \mathrm{~Hz}, 1 \mathrm{H}\right), 7.52-7.40$ (m, $2 \mathrm{H}), 6.88(\mathrm{~d}, J=3.5 \mathrm{~Hz}, 1 \mathrm{H}), 6.78(\mathrm{dd}, J=3.5,1.3 \mathrm{~Hz}, 1 \mathrm{H}), 5.54(\mathrm{brs}, 2 \mathrm{H}), 4.29(\mathrm{~d}, J=3.2 \mathrm{~Hz}, 1 \mathrm{H})$, $2.56(\mathrm{~s}, 3 \mathrm{H}), 1.94-1.86(\mathrm{~m}, 1 \mathrm{H}), 1.12(\mathrm{~d}, J=7.0 \mathrm{~Hz}, 3 \mathrm{H}),-0.02(\mathrm{~d}, J=6.8 \mathrm{~Hz}, 3 \mathrm{H}) ;{ }^{13} \mathrm{C} \mathrm{NMR}(100$ $\left.\mathrm{MHz}, \mathrm{CDCl}_{3}\right) \delta(\mathrm{ppm}) 148.0,146.5,143.5,142.9,137.8,136.8,135.3,132.3,131.5,129.6,127.9$, 127.5, 126.1 125.7, 123.0, 115.7, 90.3, 53.2, 28.5, 22.2, 15.5, 14.9; HRMS (ESI-TOF) m/z: [M + H] ${ }^{+}$ Calcd for $\left[\mathrm{C}_{22} \mathrm{H}_{19} \mathrm{~N}_{3} \mathrm{O}_{2} \mathrm{~S}+\mathrm{H}\right]^{+}$390.1271; Found 390.1272.

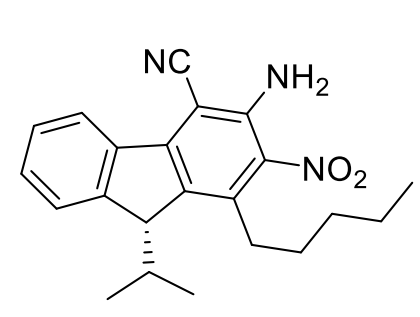

\section{(R)-3-Amino-9-isopropyl-2-nitro-1-pentyl-9H-fluorene-4-carbonitrile}

(3s): 2-(3-Isopropyl-2,3-dihydro-1H-inden-1-ylidene)malononitrile $\mathbf{1 a}$ (22.2 mg, $0.0999 \mathrm{mmol}),(E)-1$-nitrohept-1-ene $2 \mathbf{k}(28.6 \mathrm{mg}, 0.200 \mathrm{mmol}$, 2.0 equiv) and $\mathbf{C 2}(8.3 \mathrm{mg}, 0.020 \mathrm{mmol}, 20 \mathrm{~mol} \%)$ were added to a reaction tube, and the mixture was degassed and backfilled with oxygen (balloon). Dry toluene (1.0 mL) was added by syringe, and the mixture was stirred at $\mathrm{rt}$ for $48 \mathrm{~h}$. After the consumption of compound 1a, dry DCM $(1.0 \mathrm{~mL})$ was added by syringe, and the mixture was stirred for another $48 \mathrm{~h}$. After completion (monitored by TLC), the solvent was evaporated in vacuo. The residue was purified by flash chromatography on silica gel $(\mathrm{PE} / \mathrm{EtOAc}=20: 1)$ to give the product 3s: $15.0 \mathrm{mg}(0.0413 \mathrm{mmol})$ as a yellow thick oil, $41 \%$ yield; $[\alpha]_{\mathrm{D}}^{25}=-103.6\left(c=0.45 \mathrm{in} \mathrm{CHCl}_{3}\right), 75 \%$ ee, determined by HPLC analysis [Chiralpak ID, $n$-hexane $/ i$-PrOH $=95 / 5,1.0 \mathrm{~mL} / \mathrm{min}, \lambda=254 \mathrm{~nm}$, $\mathrm{t}($ minor $)=6.94 \mathrm{~min}, \mathrm{t}($ major $)=7.39 \mathrm{~min}] ;{ }^{1} \mathrm{H} \mathrm{NMR}\left(400 \mathrm{MHz}, \mathrm{CDCl}_{3}\right) \delta(\mathrm{ppm}) 8.50-8.34(\mathrm{~m}, 1 \mathrm{H})$, 7.65-7.55 (m, 1H), 7.50-7.38 (m, 2H), $5.67(\mathrm{~s}, 2 \mathrm{H}), 4.00(\mathrm{~d}, J=2.9 \mathrm{~Hz}, 1 \mathrm{H}), 3.14-2.90(\mathrm{~m}, 2 \mathrm{H})$, 2.64-2.41 (m, 1H), 1.60-1.44 (m, 3H), $1.37(\mathrm{~m}, 6 \mathrm{H}), 0.91(\mathrm{t}, J=2.9 \mathrm{~Hz}, 3 \mathrm{H}), 0.12(\mathrm{~d}, J=6.7 \mathrm{~Hz}$, $3 \mathrm{H}) ;{ }^{13} \mathrm{C}$ NMR $\left(100 \mathrm{MHz}, \mathrm{CDCl}_{3}\right) \delta(\mathrm{ppm}) 147.7,146.5,144.6,140.0,138.0,136.5,135.3,129.4$, 
127.9, 125.5, 123.0, 115.9, 52.8, 32.2, 31.0, 30.2, 29.5, 22.4, 22.2, 15.3, 13.9; HRMS (ESI-TOF) m/z: $[\mathrm{M}-\mathrm{H}]^{-}$Calcd for $\left[\mathrm{C}_{22} \mathrm{H}_{25} \mathrm{~N}_{3} \mathrm{O}_{2}-\mathrm{H}\right]^{-}$362.1874; Found 362.1879.

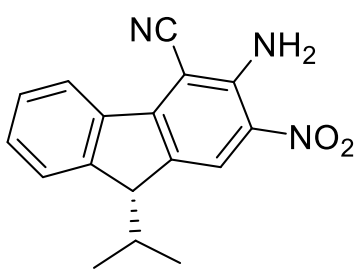

(R)-3-Amino-9-isopropyl-2-nitro-9H-fluorene-4-carbonitrile (3t): 2-(3Isopropyl-2,3-dihydro-1H-inden-1-ylidene)malononitrile $\quad \mathbf{1 a} \quad(22.2 \mathrm{mg}$, $0.0999 \mathrm{mmol}),(E)-(2-$ nitrovinyl)cyclohexane 21 (31.0 mg, $0.200 \mathrm{mmol}, 2.0$ equiv) and $\mathbf{C 2}$ ( $8.3 \mathrm{mg}, 0.020 \mathrm{mmol}, 20 \mathrm{~mol} \%$ ) were added to a reaction tube, and the mixture was degassed and backfilled with oxygen (balloon). Dry toluene (1.0 mL) was added via syringe, and the mixture was stirred at $\mathrm{rt}$ for 2 days. After the consumption of compound 1a, dry DCM (1.0 mL) was added to the mixture via syringe, and the mixture was stirred for another 2 days. After completion (monitored by TLC), the solvent was evaporated in vacuo. The residue was purified by flash chromatography on silica gel $(\mathrm{PE} / \mathrm{EtOAc}=20: 1)$ to give the product 3t: $25.8 \mathrm{mg}(0.0880$ mmol), as a pale yellow solid , 88\% yield; m.p. $127-130^{\circ} \mathrm{C} ;[\alpha]_{\mathrm{D}}{ }^{25}=-51.7\left(c=0.45\right.$ in $\left.\mathrm{CHCl}_{3}\right) 84 \%$ ee, determined by HPLC analysis [Chiralpak IE, $n$-hexane $/ i-\mathrm{PrOH}=90 / 10,1.0 \mathrm{~mL} / \mathrm{min}, \lambda=254 \mathrm{~nm}$, $\mathrm{t}($ major $)=11.28 \mathrm{~min}, \mathrm{t}($ minor $)=12.43 \mathrm{~min}] ;{ }^{1} \mathrm{H} \mathrm{NMR}\left(400 \mathrm{MHz}, \mathrm{CDCl}_{3}\right) \delta(\mathrm{ppm}) 8.48-8.45(\mathrm{~m}$, 2H), 7.62-7.59 (m, 1H), 7.55-7.48 (m, 2H), 6.95 (brs, 2H), $3.93(\mathrm{~d}, J=3.3 \mathrm{~Hz}, 1 \mathrm{H}), 2.59-2.51(\mathrm{~m}$, $1 \mathrm{H}), 0.91(\mathrm{~d}, J=6.9 \mathrm{~Hz}, 3 \mathrm{H}), 0.81(\mathrm{~d}, J=6.9 \mathrm{~Hz}, 3 \mathrm{H}) ;{ }^{13} \mathrm{C} \mathrm{NMR}\left(100 \mathrm{MHz}, \mathrm{CDCl}_{3}\right) \delta(\mathrm{ppm}) 151.0$, $150.1,146.7,137.0,134.7,131.0,130.6,128.0,126.3,125.1,123.5,115.5,91.4,52.5,32.2,19.4$, 18.5; HRMS (ESI-TOF) m/z: [M - H] $]^{-}$: Calcd for $\left[\mathrm{C}_{17} \mathrm{H}_{15} \mathrm{~N}_{3} \mathrm{O}_{2}-\mathrm{H}\right]^{-}$292.1091; Found 292.1091.

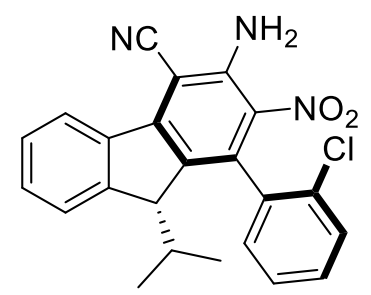

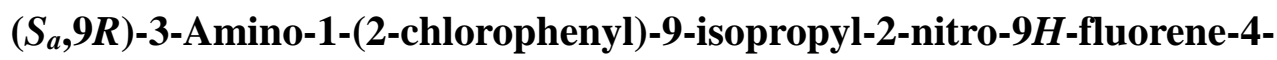
carbonitrile (3u): 2-(3-Isopropyl-2,3-dihydro-1H-inden-1-ylidene)malononitrile 1a (22.2 mg, $0.0999 \mathrm{mmol}),(E)$-1-chloro-2-(2-nitrovinyl)benzene $\mathbf{2 m}$ (36.6 mg, $0.199 \mathrm{mmol}, 2.0$ equiv) and $\mathbf{C 2}(8.3 \mathrm{mg}, 0.020 \mathrm{mmol}, 20 \mathrm{~mol} \%$ ) were added to a reaction tube, and the mixture was degassed and backfilled with oxygen (balloon). Dry toluene $(1.0 \mathrm{~mL})$ was added via syringe, and the mixture was stirred at $\mathrm{rt}$ for $10 \mathrm{~h}$. After the consumption of compound 1a, dry DCM $(1.0 \mathrm{~mL})$ was added via syringe, and the mixture was stirred for another $38 \mathrm{~h}$. After completion (monitored by TLC), the solvent was evaporated in vacuo. The residue was purified by flash chromatography on silica gel $(\mathrm{PE} / \mathrm{EtOAc}=20: 1)$ to give the product 3u: $21.8 \mathrm{mg}(0.0540 \mathrm{mmol})$, as a yellow solid, 54\% yield; m.p. $208-210{ }^{\circ} \mathrm{C} ;[\alpha]_{\mathrm{D}}{ }^{25}=-474.2(c=0.042$ 
in $\mathrm{MeOH}$ ), 18:1 dr, 85\% ee, determined by HPLC analysis [Chiralpak IC, $n$-hexane/i-PrOH = 80/20, $1.0 \mathrm{~mL} / \mathrm{min}, \lambda=254 \mathrm{~nm}, \mathrm{t}$ (minor) $=7.87 \mathrm{~min}, \mathrm{t}$ (major) $=9.84 \mathrm{~min}] ;{ }^{1} \mathrm{H} \mathrm{NMR}\left(400 \mathrm{MHz}, \mathrm{CDCl}_{3}\right) \delta$ (ppm) $8.46(\mathrm{~d}, J=7.5 \mathrm{~Hz}, 1 \mathrm{H}), 7.55(\mathrm{~d}, J=8.0 \mathrm{~Hz}, 1 \mathrm{H}), 7.53-7.34(\mathrm{~m}, 4 \mathrm{H}), 7.29(\mathrm{t}, J=7.5 \mathrm{~Hz}, 1 \mathrm{H})$, 7.12 (dd, $J=7.6,1.4 \mathrm{~Hz}, 1 \mathrm{H}), 5.98$ (brs, 2H), 3.93 (d, $J=3.4 \mathrm{~Hz}, 1 \mathrm{H}), 1.64-1.57$ (m, 1H), 0.98 (d, $J$ $=7.0 \mathrm{~Hz}, 3 \mathrm{H}),-0.01(\mathrm{~d}, J=6.8 \mathrm{~Hz}, 3 \mathrm{H}) ;{ }^{13} \mathrm{C} \mathrm{NMR}\left(100 \mathrm{MHz}, \mathrm{CDCl}_{3}\right) \delta(\mathrm{ppm}) 148.3,147.3,144.8$, 137.6, 136.33, 136.27, 134.6, 133.8, 132.8, 130.2, 130.1, 129.9, 128.7, 127.9, 126.9, 125.9, 123.2, 115.6, 90.9, 53.1, 29.4, 22.0, 15.1; HRMS (ESI-TOF) m/z: $[\mathrm{M}+\mathrm{Na}]^{+}$Calcd for $\left[\mathrm{C}_{23} \mathrm{H}_{18}{ }^{35} \mathrm{ClN}_{3} \mathrm{O}_{2}+\right.$ $\mathrm{Na}]^{+}$426.0980; Found 426.0972; Calcd for $\left[\mathrm{C}_{23} \mathrm{H}_{18}{ }^{37} \mathrm{ClN}_{3} \mathrm{O}_{2}+\mathrm{Na}\right]^{+}$428.0951; Found 428.0954.

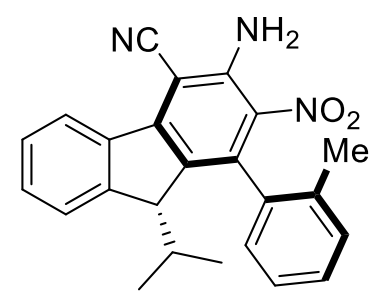

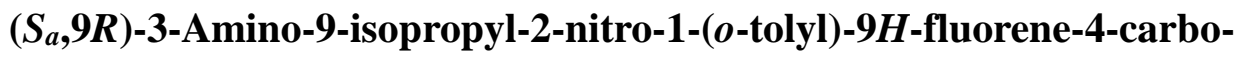
nitrile (3v): 2-(3-Isopropyl-2,3-dihydro-1H-inden-1-ylidene) malononitrile 1a (22.2 mg, $0.0999 \mathrm{mmol}),(E)-1-m e t h y l-2-(2-n i t r o v i n y l)$ benzene $2 \mathrm{n}$ (32.6 $\mathrm{mg}, 0.200 \mathrm{mmol}, 2.0$ equiv) and $\mathbf{C 2}(8.3 \mathrm{mg}, 0.020 \mathrm{mmol}, 20 \mathrm{~mol} \%)$ were added to a reaction tube, and the mixture was degassed and backfilled with oxygen (balloon). Dry toluene $(1.0 \mathrm{~mL})$ was added via syringe, and the mixture was stirred at $\mathrm{rt}$ for $10 \mathrm{~h}$. After the consumption of compound 1a, dry DCM $(1.0 \mathrm{~mL})$ was added via syringe, and the mixture was stirred for another $38 \mathrm{~h}$. After completion (monitored by TLC), the solvent was evaporated in vacuo. The residue was purified by flash chromatography on silica gel $(\mathrm{PE} / \mathrm{EtOAc}=20: 1)$ to give the product 3v: $24.1 \mathrm{mg}(0.0628)$, as a pale yellow solid, 63\% yield; m.p. $179-181{ }^{\circ} \mathrm{C} ;[\alpha]_{\mathrm{D}}{ }^{25}=-95.2(c=0.082$ in $\mathrm{MeOH}),>20: 1 \mathrm{dr}, 93 \%$ ee, determined by HPLC analysis [Chiralpak IB, $n$-hexane $/ i$-PrOH = 80/20, $1.0 \mathrm{~mL} / \mathrm{min}, \lambda=254 \mathrm{~nm}, \mathrm{t}$ (minor) $=5.02 \mathrm{~min}, \mathrm{t}$ (major) $=5.24 \mathrm{~min}] ;{ }^{1} \mathrm{H} \mathrm{NMR}\left(400 \mathrm{MHz}, \mathrm{CDCl}_{3}\right) \delta$ (ppm) $8.49(\mathrm{~d}, J=7.7 \mathrm{~Hz}, 1 \mathrm{H}), 7.54-7.41(\mathrm{~m}, 3 \mathrm{H}), 7.38-7.31(\mathrm{~m}, 2 \mathrm{H}), 7.22-7.18(\mathrm{~m}, 1 \mathrm{H}), 7.01(\mathrm{~d}$, $J=7.6 \mathrm{~Hz}, 1 \mathrm{H}), 5.78$ (brs, 2H), $3.68(\mathrm{~d}, J=3.5 \mathrm{~Hz}, 1 \mathrm{H}), 2.19$ (s, 3H), 1.75-1.67 (m, 1H), $0.98(\mathrm{~d}, J$ $=7.0 \mathrm{~Hz}, 3 \mathrm{H}), 0.01(\mathrm{~d}, J=6.7 \mathrm{~Hz}, 3 \mathrm{H}) ;{ }^{13} \mathrm{C} \mathrm{NMR}\left(100 \mathrm{MHz}, \mathrm{CDCl}_{3}\right) \delta(\mathrm{ppm}) 148.1,146.6,144.2$, 138.7, 137.8, 135.82, 135.77, 134.9, 134.6, 130.6, 129.6, 128.8, 127.9, 127.0, 125.9, 125.8, 123.1, 115.7, 90.2, 53.3, 29.2, 22.0, 19.9, 15.0; HRMS (ESI-TOF) $\mathrm{m} / \mathrm{z}:[\mathrm{M}+\mathrm{Na}]^{+}$Calcd for $\left[\mathrm{C}_{24} \mathrm{H}_{21} \mathrm{~N}_{3} \mathrm{O}_{2}+\right.$ $\mathrm{Na}]^{+}$406.1526; Found 406.1524. 


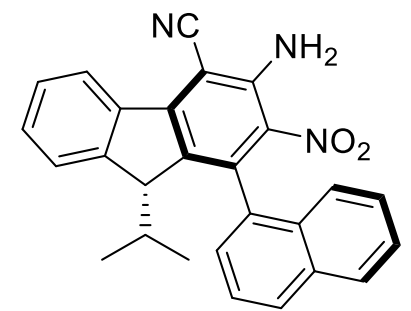

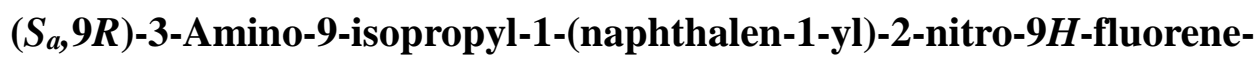

4-carbonitrile (3w): 2-(3-Isopropyl-2,3-dihydro-1H-inden-1-ylidene) malononitrile 1a $(22.2 \mathrm{mg}, 0.0999 \mathrm{mmol}),(E)$-1-(2-nitrovinyl)naphthalene 20 (39.8 mg, $0.200 \mathrm{mmol}, 2.0$ equiv) and C2 (8.3 mg, $0.020 \mathrm{mmol}, 20$ mol \%) were added to a reaction tube, and the mixture was degassed and backfilled with oxygen (balloon). Dry toluene $(1.0 \mathrm{~mL})$ was added by syringe, and the mixture was stirred at $\mathrm{rt}$ for $10 \mathrm{~h}$. After the consumption of compound 1a, dry DCM $(1.0 \mathrm{~mL})$ was added by syringe, and the mixture was stirred for another $38 \mathrm{~h}$. After completion (monitored by TLC), the solvent was evaporated in vacuo. The residue was purified by flash chromatography on silica gel $(\mathrm{PE} / \mathrm{EtOAc}=20: 1)$ to give the product 3w: $28.1 \mathrm{mg}(0.0670 \mathrm{mmol})$, as a pale yellow solid, $67 \%$ yield; m.p. $228-230^{\circ} \mathrm{C} ;[\alpha]_{\mathrm{D}}{ }^{25}=-332.0(c$ $=0.067$ in $\mathrm{MeOH}), 15: 1 \mathrm{dr}, 90 \%$ ee, determined by HPLC analysis [Chiralpak IC, $n$-hexane $/ i$-PrOH $=80 / 20,1.0 \mathrm{~mL} / \mathrm{min}, \lambda=254 \mathrm{~nm}, \mathrm{t}$ (minor) $=9.19 \mathrm{~min}, \mathrm{t}$ (major $)=10.76 \mathrm{~min}] ;{ }^{1} \mathrm{H} \mathrm{NMR}(400 \mathrm{MHz}$, $\left.\mathrm{CDCl}_{3}\right) \delta(\mathrm{ppm}) 8.54(\mathrm{~d}, J=7.8 \mathrm{~Hz}, 1 \mathrm{H}), 7.97(\mathrm{dd}, J=8.3,3.5 \mathrm{~Hz}, 2 \mathrm{H}), 7.67-7.36(\mathrm{~m}, 7 \mathrm{H}), 7.30-7.28$ (m, 1H), 5.87 (brs, 2H), $3.58(\mathrm{~d}, J=3.4 \mathrm{~Hz}, 1 \mathrm{H}), 1.47-1.38(\mathrm{~m}, 1 \mathrm{H}), 0.72(\mathrm{~d}, J=7.1 \mathrm{~Hz}, 3 \mathrm{H}),-0.01$ $(\mathrm{d}, J=6.8 \mathrm{~Hz}, 3 \mathrm{H}) ;{ }^{13} \mathrm{C} \mathrm{NMR}\left(100 \mathrm{MHz}, \mathrm{CDCl}_{3}\right) \delta(\mathrm{ppm}) 148.2,146.5,144.4,137.7,137.6,136.6$, 135.5, 133.8, 133.1, 130.6, 129.6, 129.3, 128.7, 127.8, 126.9, 126.4, 125.8, 125.5, 125.2, 124.7, 123.1, 115.7, 90.5, 53.5, 29.1, 21.7, 14.8; HRMS (ESI-TOF) m/z: $[\mathrm{M}+\mathrm{Na}]^{+} \mathrm{Calcd}$ for $\left[\mathrm{C}_{27} \mathrm{H}_{21} \mathrm{~N}_{3} \mathrm{O}_{2}+\mathrm{Na}\right]^{+}$ 442.1526; Found 442.1528.

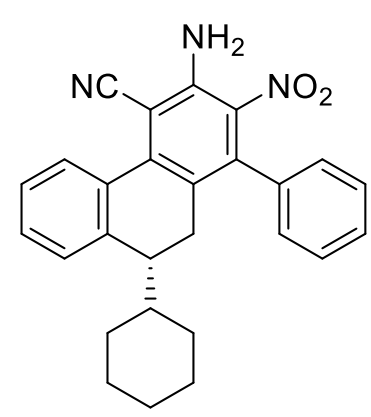

(R)-3-Amino-9-cyclohexyl-2-nitro-1-phenyl-9,10-dihydrophenanthrene4-carbonitrile (5): 2-(4-Cyclohexyl-3,4-dihydronaphthalen-1(2H)-ylidene) malononitrile 4 (27.6 mg, $0.0999 \mathrm{mmol}), \beta$-nitro styrene $\mathbf{2 a}(29.8 \mathrm{mg}, 0.200$ mmol, 2.0 equiv) and $\mathbf{C 2}(8.3 \mathrm{mg}, 0.020 \mathrm{mmol}, 20 \mathrm{~mol} \%)$ were added to a reaction tube, and the mixture was degassed and backfilled with oxygen (balloon). Dry toluene (1.0 mL) was added via syringe, and the mixture was stirred at $\mathrm{rt}$ for 2 days. After the consumption of compound 4, dry DCM (1.0 mL) was added to the mixture via syringe, and the mixture was stirred for another 2 days. After completion (monitored by TLC), the solvent was evaporated in vacuo. The residue was purified by flash chromatography on silica gel $(\mathrm{PE} / \mathrm{EtOAc}=20: 1)$ to give the product 5: $17.7 \mathrm{mg}(0.0418 \mathrm{mmol})$, as pale yellow solid, $42 \%$ yield; m.p. $209-211^{\circ} \mathrm{C} ;[\alpha]_{\mathrm{D}}{ }^{25}=-28.8\left(c=0.125\right.$ in $\left.\mathrm{CHCl}_{3}\right), 58 \%$ ee, determined by HPLC analysis 
[Chiralpak AD-H, $n$-hexane $/ i$-PrOH $=90 / 10,1.0 \mathrm{~mL} / \mathrm{min}, \lambda=254 \mathrm{~nm}, \mathrm{t}$ (minor) $=5.07$, $\mathrm{t}$ (major) $=$ $7.13 \mathrm{~min}] ;{ }^{1} \mathrm{H} \mathrm{NMR}\left(400 \mathrm{MHz}, \mathrm{CDCl}_{3}\right) \delta(\mathrm{ppm}) \delta 8.23(\mathrm{dd}, J=7.3,1.7 \mathrm{~Hz}, 1 \mathrm{H}), 7.48-7.34(\mathrm{~m}, 5 \mathrm{H})$, 7.26-7.22 (m, 1H), $7.17(\mathrm{dd}, J=7.0,1.9 \mathrm{~Hz}, 1 \mathrm{H}), 7.14-7.07(\mathrm{~m}, 1 \mathrm{H}), 5.71(\mathrm{brs}, 2 \mathrm{H}), 2.61(\mathrm{dd}, J=$ 15.9, $2.4 \mathrm{~Hz}, 1 \mathrm{H}), 2.43$ (dd, $J=15.9,4.9 \mathrm{~Hz}, 1 \mathrm{H}), 2.36-2.23(\mathrm{~m}, 1 \mathrm{H}), 1.59-1.51$ (m, 3H), 1.47-1.38 (m, 1H), 1.35-1.28 (m, 1H), 1.06-0.92 (m, 4H), 0.90-0.79 (m, 1H), 0.67-0.47 (m, 1H); ${ }^{13} \mathrm{C}$ NMR $\left(100 \mathrm{MHz}, \mathrm{CDCl}_{3}\right) \delta(\mathrm{ppm}) 143.3,142.8,142.7,140.9,135.7,13.5,130.3,129.9,129.5,129.1,128.8$, $128.55,128.53,127.6,127.0,126.7,126.2,117.3,44.9,38.0,31.8,31.0,28.3,26.2,26.0,25.9$; HRMS (ESI-TOF) m/z: [M - H $]^{-}$Calcd for $\left[\mathrm{C}_{27} \mathrm{H}_{25} \mathrm{~N}_{3} \mathrm{O}_{2}-\mathrm{H}\right]^{-} 422.1874$; Found 422.1870.

\section{Unsuccessful substrates:}

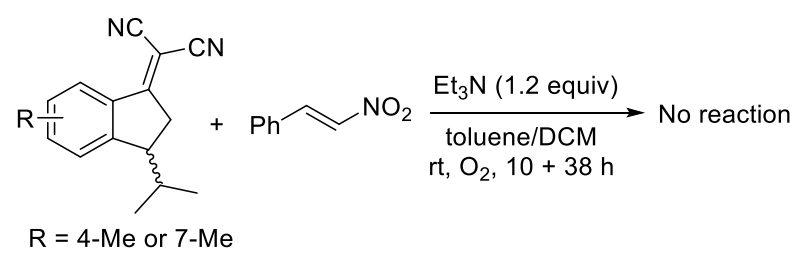

In addition, 4-methyl or 7-methyl-substituted $\alpha, \alpha$-dicyanoolefins was tested, but no desired addition products were observed even in the presence of excess $\mathrm{NEt}_{3}$, probably because of the steric hindrance of these substrates which rendered Michael addition step unfavorable.

\section{Proposed mechanism for the formation of dealkylation product $3 t$}

The nitroolefin with a branched alkyl group, such as cyclohexanaldehyde or even isobutyraldehyde derived nitroolefin, reacted with $\alpha, \alpha$-dicyanoolefin $\mathbf{1 a}$ to give a dealkylation product 3t, but also with promising enantioselectivity (Scheme S1a). When TEMPO (2,2,6,6tetramethylpiperidinyloxy) was added to the reaction mixture, the yield of dealkylation product $\mathbf{3 t}$ was significantly decreased, but the normal product $\mathbf{3 \mathbf { t } ^ { \prime }}$ and the corresponding intermediate $\mathbf{3 \mathbf { t } ^ { \prime \prime }}$ were isolated in fair yields (Scheme S1b), which suggested that the formation of dealkylation product 3t probably involving an oxidative radical cleavage process.

Based on the the literature reports and above control experiment, a plausible reaction mechanism was proposed, probably involving the oxidative formation of radical intermediates $\mathbf{D}$ and $\mathbf{E}$ from chiral intermediate $\mathbf{C}$, as outlined in Scheme S1c. A Hock-type rearrangement of intermediate F would give the alcohol $\mathbf{G}$, which finally delivered the observed product $\mathbf{3 t}$ after dehydration (Scheme S1c). 
a) Dealkylation product for branched nitroolefins:
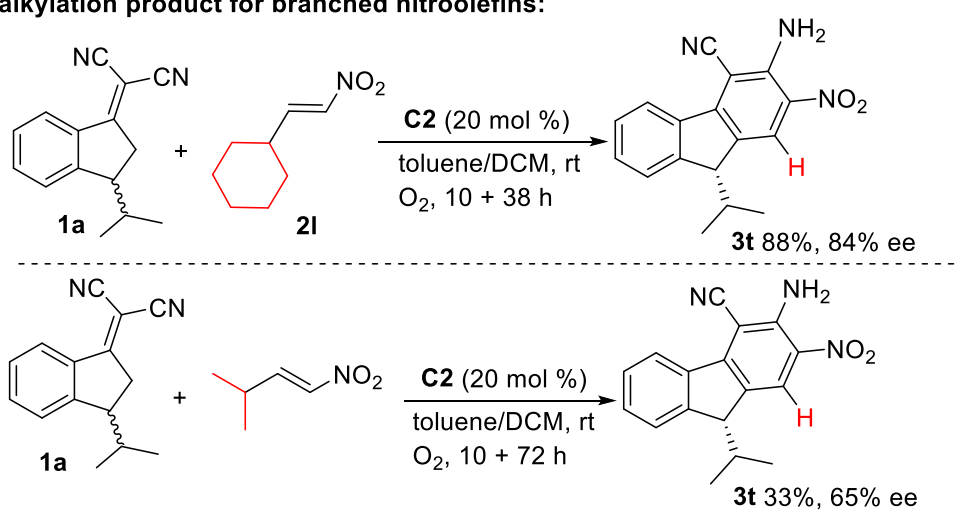

b) Control experiment:

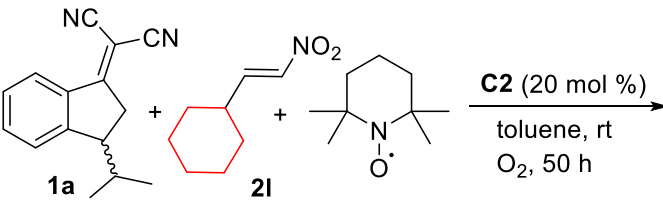

c) Proposed reaction mechanism:

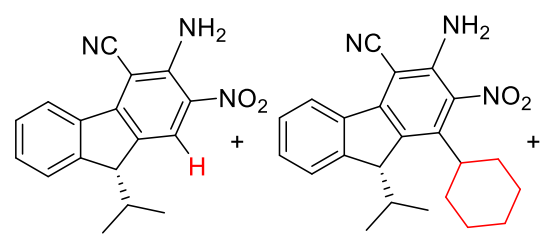

3t $17 \%$

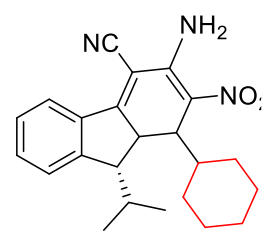

$3 t " 24 \%$

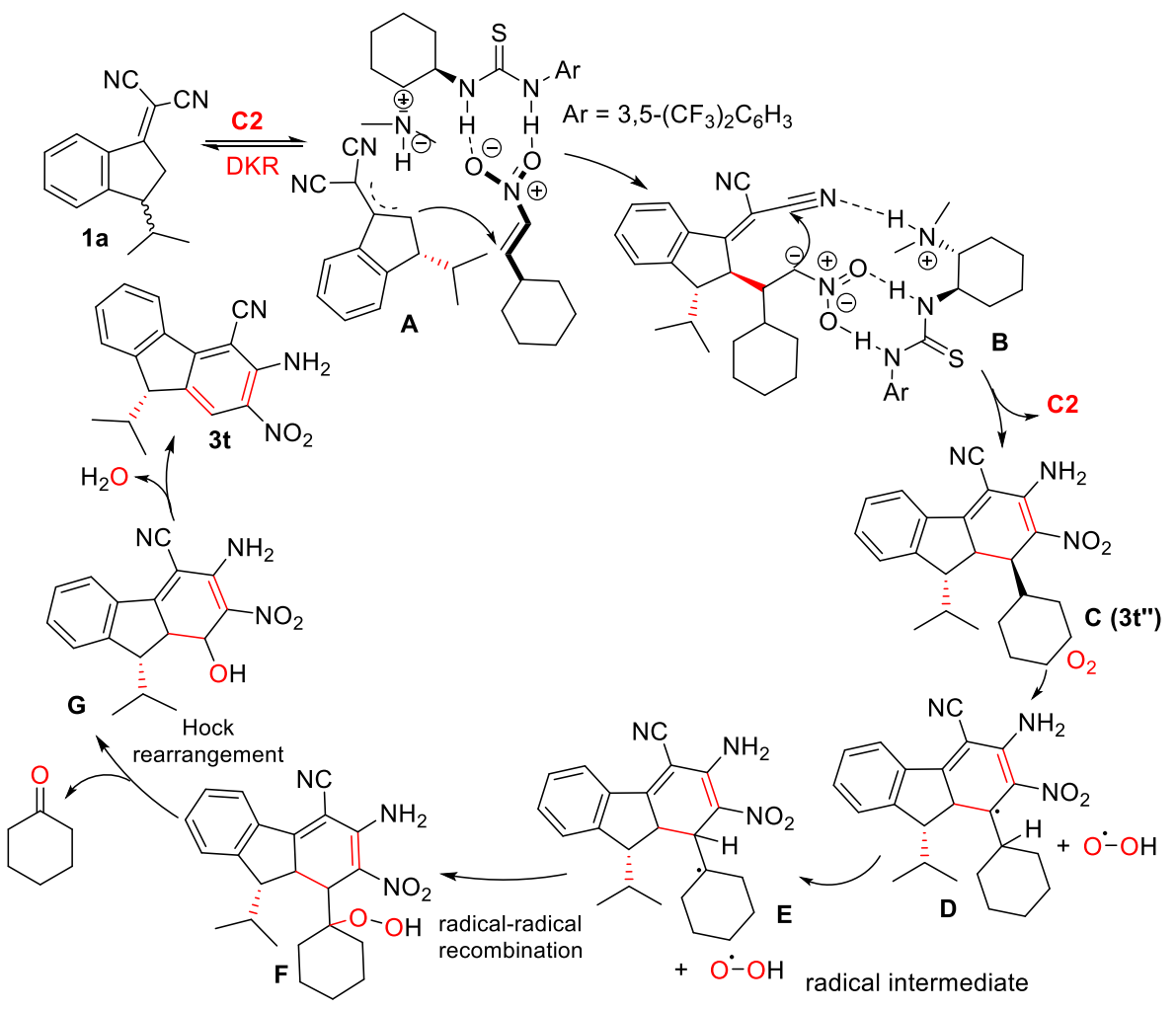

(1) Chen, K.; Zhang, P.; Wang, Y.; Li, H. Green Chem. 2014, 16, 2344. (2) Taniguchi, T. Synlett 2020, 10.1055/s-0040-1707240. (3) Weber, M.; Daldrup, J.-B. G.; Weber, M. Noncatalyzed radical chain oxidation: Cumene hydroperoxide in liquid phase aerobic nxidation catalvsis· Industrial annlications and ac:ademir, nersners.tives. Wilev-VC.H· Weinheim 201615

\section{Scheme S1 Dealkylation for branched nitroolefins and proposed mechanism}

Procedure for control experiment: 2-(3-Isopropyl-2,3-dihydro-1 $H$-inden-1-ylidene)malononitrile $1 \mathrm{a}(44.4 \mathrm{mg}, 0.200 \mathrm{mmol}),(E)-(2$-nitrovinyl)cyclohexane $2 \mathbf{l}(62.0 \mathrm{mg}, 0.415 \mathrm{mmol}, 2.08$ equiv), TEMPO (40.6 mg, $0.260 \mathrm{mmol}, 1.3$ equiv) and C2 (16.5 mg, $0.0399 \mathrm{mmol}, 20 \mathrm{~mol} \%$ ) were added 
to a reaction tube, and the mixture was degassed and backfilled with oxygen (balloon). Dry toluene $(2.0 \mathrm{~mL})$ was added via syringe, and the mixture was stirred at $\mathrm{rt}$ for $50 \mathrm{~h}$. After the consumption of compound 1a (monitored by TLC), the solvent was evaporated in vacuo. The residue was purified by flash chromatography on silica gel $(\mathrm{PE} / \mathrm{EtOAc}=20: 1)$ to give the following products:

3t: $10.2 \mathrm{mg}$ (0.0348 mmol), as a pale yellow solid, $17 \%$ yield.

3t': $37.0 \mathrm{mg}$ (0.0987 mmol), as a pale yellow solid, $49 \%$ yield; m.p. $185-187{ }^{\circ} \mathrm{C} ;{ }^{1} \mathrm{H} \mathrm{NMR}(600 \mathrm{MHz}$, $\left.\mathrm{CDCl}_{3}\right) \delta(\mathrm{ppm}) 8.39(\mathrm{~d}, J=7.7 \mathrm{~Hz}, 1 \mathrm{H}), 7.57(\mathrm{~d}, J=7.5 \mathrm{~Hz}, 1 \mathrm{H}), 7.52-7.34(\mathrm{~m}, 2 \mathrm{H}), 4.85(\mathrm{brs}, 2 \mathrm{H})$, $4.01(\mathrm{~d}, J=2.9 \mathrm{~Hz}, 1 \mathrm{H}), 3.09-2.95(\mathrm{~m}, 1 \mathrm{H}), 2.53-2.40(\mathrm{~m}, 1 \mathrm{H}), 1.97-1.82(\mathrm{~m}, 4 \mathrm{H}), 1.81-1.70(\mathrm{~m}$, $3 \mathrm{H}), 1.38(\mathrm{~d}, J=7.0 \mathrm{~Hz}, 3 \mathrm{H}), 1.37-1.25(\mathrm{~m}, 3 \mathrm{H}), 0.12(\mathrm{~d}, J=6.7 \mathrm{~Hz}, 3 \mathrm{H}) ;{ }^{13} \mathrm{C} \mathrm{NMR}(150 \mathrm{MHz}$, $\left.\mathrm{CDCl}_{3}\right) \delta(\mathrm{ppm}) 147.0,144.9,142.8,140.9,138.3,137.8,137.1,128.9,127.8,125.3,122.5,115.8$, 89.4, 52.9, 43.2, 31.5, 31.3, 30.5, 27.7, 27.3, 25.7, 22.4, 15.2; HRMS (ESI-TOF) m/z: [M - H] $]^{-}$Calcd for $\left[\mathrm{C}_{23} \mathrm{H}_{25} \mathrm{~N}_{3} \mathrm{O}_{2}-\mathrm{H}\right]^{-}$374.1874; Found 374.1872.

3t": $17.8 \mathrm{mg}$ (0.0472 mmol), as a pale yellow solid, 24\% yield; m.p. 173-175 ${ }^{\circ} \mathrm{C} ;{ }^{1} \mathrm{H}$ NMR (600 $\left.\mathrm{MHz} \mathrm{CDCl}_{3}\right) \delta(\mathrm{ppm}) 8.12(\mathrm{~d}, J=7.8 \mathrm{~Hz}, 1 \mathrm{H}), 7.60-7.48(\mathrm{~m}, 2 \mathrm{H}), 7.46-7.36(\mathrm{~m}, 1 \mathrm{H}), 3.65(\mathrm{t}, J=$ $2.8 \mathrm{~Hz}, 1 \mathrm{H}), 3.16-2.97$ (m, 2H), 2.31-2.20 (m, 1H), 1.83-1.69 (m, 3H), 1.69-1.52 (m, 4H), 1.34 (d, $J=7.2 \mathrm{~Hz}, 3 \mathrm{H}), 1.29-1.24(\mathrm{~m}, 1 \mathrm{H}), 1.22-1.14(\mathrm{~m}, 1 \mathrm{H}), 1.14-1.01(\mathrm{~m}, 2 \mathrm{H}), 0.99(\mathrm{~d}, J=7.0 \mathrm{~Hz}, 3 \mathrm{H})$, $0.94(\mathrm{dd}, \mathrm{J}=12.5,3.6 \mathrm{~Hz}, 1 \mathrm{H}) ;{ }^{13} \mathrm{C} \mathrm{NMR}\left(150 \mathrm{MHz}, \mathrm{CDCl}_{3}\right) \delta(\mathrm{ppm}) 171.5,147.1,146.6,136.5$, 133.1, 127.9, 126.0, 124.5, 117.2, 114.0, 96.1, 54.5, 51.3, 43.5, 38.9, 29.2, 28.3, 27.0, 26.6, 26.5, 26.3, 22.5, 16.3; HRMS (ESI-TOF) m/z: $[\mathrm{M}+\mathrm{Na}]^{+}$Calcd for $\left[\mathrm{C}_{23} \mathrm{H}_{27} \mathrm{~N}_{3} \mathrm{O}_{2}+\mathrm{Na}\right]^{+}$400.1996; Found 400.1994 .

\section{Transformations of the product $3 a$}

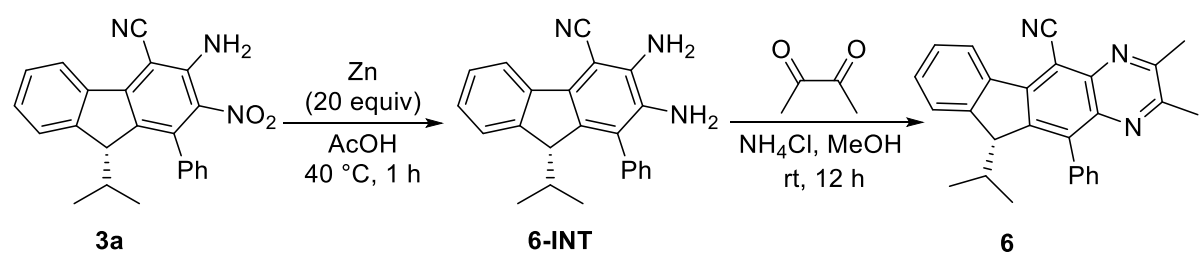

3a (20.6 mg, $0.0558 \mathrm{mmol})$, zinc powder (72.8 mg, $1.12 \mathrm{mmol}, 20.0$ equiv) were added to HOAc $(2.0 \mathrm{~mL})$, and the resulting mixture was stirred at $40{ }^{\circ} \mathrm{C}$ for $1 \mathrm{~h}$. After completion, the mixture was concentrated, and diluted with EtOAc $(20 \mathrm{~mL})$, and filter through a pad of celite, the filtrate was washed with saturated $\mathrm{Na}_{2} \mathrm{CO}_{3}(10 \mathrm{~mL})$ carefully, and dried over $\mathrm{Na}_{2} \mathrm{SO}_{4}$. The solvent was evaporated, and the residue was purified by column chromatography $(\mathrm{PE} / \mathrm{EtOAc}=5: 1)$ to give 6-INT: $19.0 \mathrm{mg}$ 
(0.0559 mmol), as a brown oil.

6-INT (19.0 mg, $0.0559 \mathrm{mmol})$, biacetyl (6.3 mg, $0.073 \mathrm{mmol}, 1.3$ equiv), $\mathrm{NH}_{4} \mathrm{Cl}$ ( $1.5 \mathrm{mg}, 0.028$ mmol, 0.5 equiv) were added to methanol $(0.28 \mathrm{~mL})$, the resulting mixture was stirred at $\mathrm{rt}$ for $12 \mathrm{~h}$. Then water was added $(5 \mathrm{~mL})$, and extracted with DCM $(3 \times 10 \mathrm{~mL})$, the organic phases were combined, washed with brine and dried over $\mathrm{Na}_{2} \mathrm{SO}_{4}$. After concentration, the residue was purified by column chromatography $(\mathrm{PE} / \mathrm{EtOAc}=20: 1)$ to give $6: 17.8 \mathrm{mg}(0.0457 \mathrm{mmol})$, as a yellow solid, 82\% yield; m.p. $220-222{ }^{\circ} \mathrm{C} ;[\alpha]_{\mathrm{D}}{ }^{25}=-229.4\left(c=0.51\right.$ in $\left.\mathrm{CHCl}_{3}\right), 92 \%$ ee, determined by HPLC analysis [Chiralpak AD-H, $n$-hexane $/ i-\mathrm{PrOH}=90 / 10,1.0 \mathrm{~mL} / \mathrm{min}, \lambda=254 \mathrm{~nm}, \mathrm{t}$ (minor) $=5.46, \mathrm{t}$ $($ major $)=6.42 \mathrm{~min}] ;{ }^{1} \mathrm{H} \mathrm{NMR}\left(400 \mathrm{MHz}, \mathrm{CDCl}_{3}\right) \delta(\mathrm{ppm}) \delta 8.77(\mathrm{~d}, J=7.8 \mathrm{~Hz}, 1 \mathrm{H}), 7.64-7.41(\mathrm{~m}$, $8 \mathrm{H}), 4.46(\mathrm{~d}, J=3.3 \mathrm{~Hz}, 1 \mathrm{H}), 2.80(\mathrm{~s}, 3 \mathrm{H}), 2.64(\mathrm{~s}, 3 \mathrm{H}), 1.96-1.81(\mathrm{~m}, 1 \mathrm{H}), 1.04(\mathrm{~d}, J=7.0 \mathrm{~Hz}, 3 \mathrm{H})$, $-0.04(\mathrm{~d}, J=6.8 \mathrm{~Hz}, 3 \mathrm{H}) ;{ }^{13} \mathrm{C} \mathrm{NMR}\left(100 \mathrm{MHz}, \mathrm{CDCl}_{3}\right) \delta(\mathrm{ppm}) 154.4,153.6,147.2,146.6,146.1$, $141.8,140.5,138.5,135.9,129.6,128.15,128.10,128.0,125.8,123.9,116.6,52.7,28.9,23.3,23.1$, 22.1, 15.1; HRMS (ESI-TOF) m/z: $[\mathrm{M}+\mathrm{Na}]^{+}$Calcd for $\left[\mathrm{C}_{27} \mathrm{H}_{23} \mathrm{~N}_{3}+\mathrm{Na}\right]^{+} 412.1785$; Found 412.1788 .

\section{Crystal data and structural refinement for enantiopure $3 \mathrm{~m}$ and $3 \mathrm{u}$}

Procedure for the recrystallization of 3m: To a $10 \mathrm{~mL}$ tube containing $\mathbf{3 m}$ (30 mg) were added $n$ hexane $(1.8 \mathrm{~mL})$ and EtOAc $(0.3 \mathrm{~mL})$. The mixture was heated until a clear solution was formed, which was kept aside at room temperature to obtain crystals. The crystals were subjected for single crystal XRD to determine the absolute configuration of $\mathbf{3 m}$. The data were collected by an Agilent Gemini equipped with a $\mathrm{Cu}$ radiation source $(\mathrm{K} \alpha=1.54184 \AA)$ at 294.9(2) K. CCDC 2035933 (3m) contains the supplementary crystallographic data for this paper. These data can be obtained free of charge via www.ccdc.cam.ac.uk/data_request/cif.
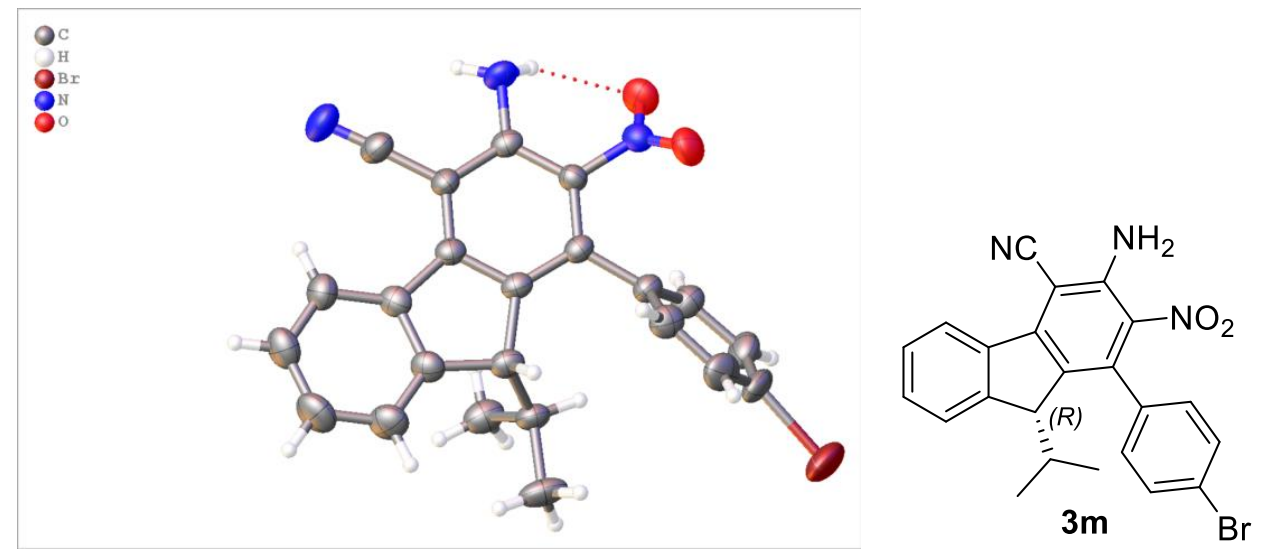

(ellipsoid contour probability 50\%) 
Identification code

Empirical formula

Formula weight

Temperature/K

Crystal system

Space group

$\mathrm{a} / \AA$

$\mathrm{b} / \AA$

$\mathrm{c} / \AA$

$\alpha{ }^{\circ}$

$\beta /{ }^{\circ}$

$\gamma /{ }^{\circ}$

Volume/ $\AA^{3}$

$\mathrm{Z}$

$\rho_{\text {calc }} \mathrm{g} / \mathrm{cm}^{3}$

$\mu / \mathrm{mm}^{-1}$

$\mathrm{F}(000)$

Crystal size $/ \mathrm{mm}^{3}$

Radiation

$2 \Theta$ range for data collection/ ${ }^{\circ}$

Index ranges

Reflections collected

Independent reflections

Data/restraints/parameters

Goodness-of-fit on $\mathrm{F}^{2}$

Final R indexes $[\mathrm{I}>=2 \sigma(\mathrm{I})]$

Final $\mathrm{R}$ indexes [all data]

Largest diff. peak/hole / e $\AA^{-3}$

Flack parameter
$3 \mathbf{m}$

$\mathrm{C}_{23} \mathrm{H}_{18} \mathrm{BrN}_{3} \mathrm{O}_{2}$

448.31

294.9(2)

monoclinic

$\mathrm{P} 21$

$10.4522(4)$

$6.5517(5)$

14.7191(7)

90

92.010(4)

90

1007.34(9)

2

1.478

2.979

456.0

$0.7 \times 0.4 \times 0.2$

$\mathrm{CuK \alpha}(\lambda=1.54184)$

8.464 to 145.946

$-12 \leq \mathrm{h} \leq 12,-6 \leq \mathrm{k} \leq 7,-18 \leq 1 \leq 13$

10163

$3416\left[R_{\text {int }}=0.0375, R_{\text {sigma }}=0.0325\right]$

$3416 / 1 / 265$

1.090

$\mathrm{R}_{1}=0.0617, \mathrm{wR}_{2}=0.1514$

$\mathrm{R}_{1}=0.0641, \mathrm{wR}_{2}=0.1563$

$0.46 /-0.95$

$-0.002(12)$

Procedure for the recrystallization of $3 \mathbf{u}$ : To a $10 \mathrm{~mL}$ tube containing $\mathbf{3 m}$ (35 mg) were added $n$ hexane $(1.8 \mathrm{~mL})$ and EtOAc $(0.3 \mathrm{~mL})$. The mixture was heated until a clear solution was formed, which was kept aside at room temperature to obtain crystals. The crystals were subjected for single crystal XRD to determine the absolute configuration of $\mathbf{3 u}$. The data were collected by an Agilent Gemini equipped with a $\mathrm{Cu}$ radiation source $(\mathrm{K} \alpha=1.54184 \AA$ ) at 295.6(2) K. CCDC 2035934 (3u) contains the supplementary crystallographic data for this paper. These data can be obtained free of charge via www.ccdc.cam.ac.uk/data_request/cif. 

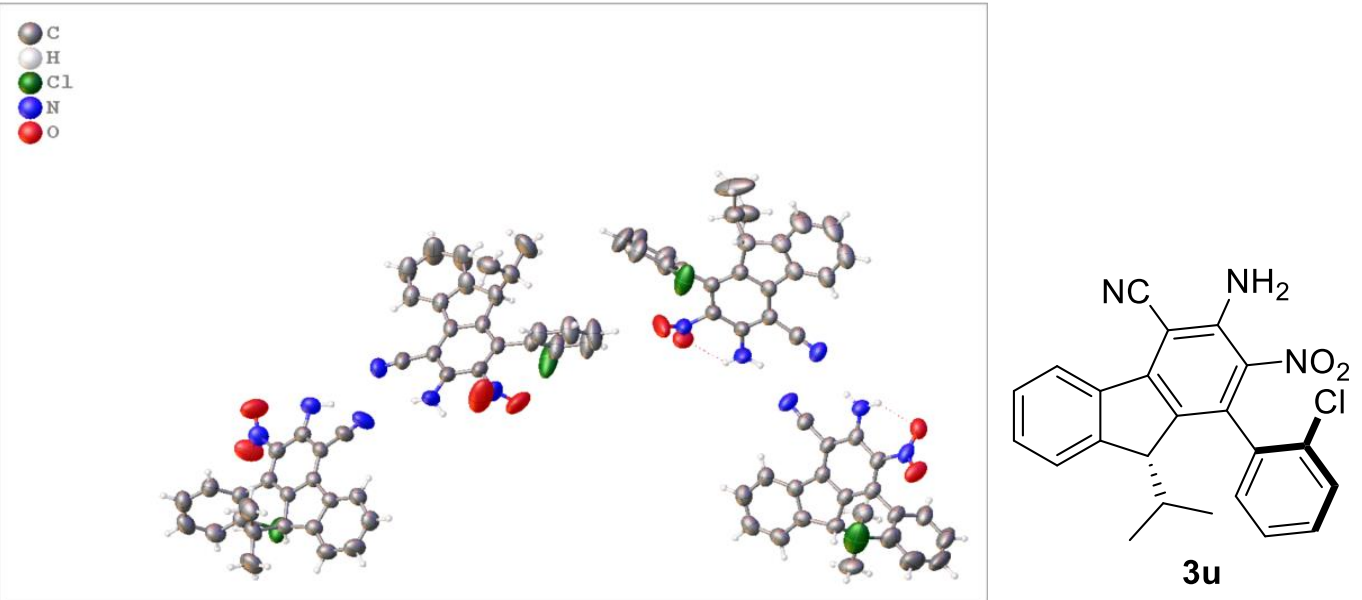

(ellipsoid contour probability 50\%)

Identification code

Empirical formula

Formula weight

Temperature/K

Crystal system

Space group

$\mathrm{a} / \AA$

$\mathrm{b} / \AA$

$\mathrm{c} / \AA$

$\alpha /^{\circ}$

$\beta /{ }^{\circ}$

$\gamma /{ }^{\circ}$

Volume $/ \AA^{3}$

$\mathrm{Z}$

$\rho_{\text {calc }} \mathrm{g} / \mathrm{cm}^{3}$

$\mu / \mathrm{mm}^{-1}$

$\mathrm{F}(000)$

Crystal size $/ \mathrm{mm}^{3}$

Radiation

$2 \Theta$ range for data collection/ ${ }^{\circ}$

Index ranges

Reflections collected

Independent reflections

Data/restraints/parameters

Goodness-of-fit on $\mathrm{F}^{2}$

Final $\mathrm{R}$ indexes $[\mathrm{I}>=2 \sigma(\mathrm{I})]$

Final R indexes [all data]

Largest diff. peak/hole / e $\AA^{-3}$

Flack parameter 3u

$\mathrm{C}_{23} \mathrm{H}_{18} \mathrm{ClN}_{3} \mathrm{O}_{2}$

403.85

295.6(2)

monoclinic

$\mathrm{P} 21$

14.7007(3)

9.6106(2)

29.9231(6)

90

102.636(2)

90

4125.23(16)

8

1.301

1.832

1680.0

$0.7 \times 0.35 \times 0.3$

$\operatorname{CuK} \alpha(\lambda=1.54184)$

9.086 to 145.976

$-18 \leq \mathrm{h} \leq 17,-11 \leq \mathrm{k} \leq 11,-36 \leq 1 \leq 35$

43677

$15229\left[\mathrm{R}_{\text {int }}=0.0363, \mathrm{R}_{\text {sigma }}=0.0325\right]$

$15229 / 1 / 1055$

1.025

$\mathrm{R}_{1}=0.0603, \mathrm{wR}_{2}=0.1690$

$\mathrm{R}_{1}=0.0674, \mathrm{wR}_{2}=0.1804$

$0.48 /-0.42$

$0.015(7)$ 
7. NMR, HRMS spectra and HPLC chromatograms

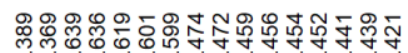

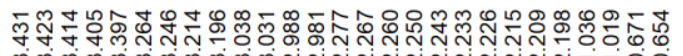

:
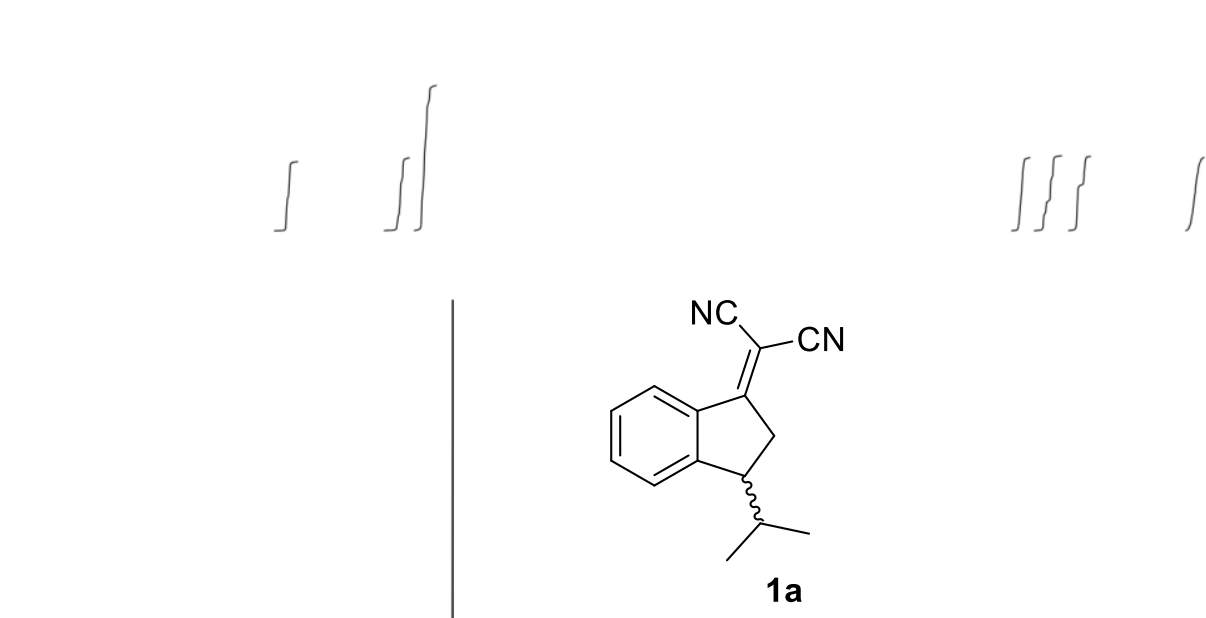

${ }^{1} \mathrm{H}$ NMR $\left(400 \mathrm{MHz}, \mathrm{CDCl}_{3}\right)$

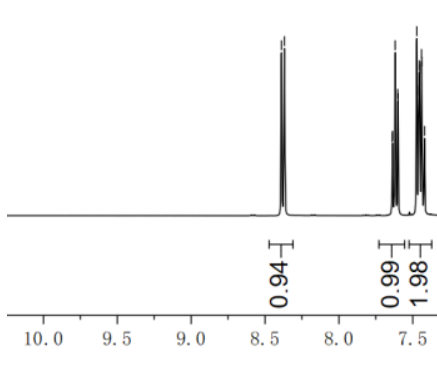

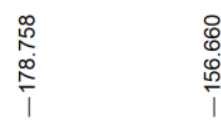

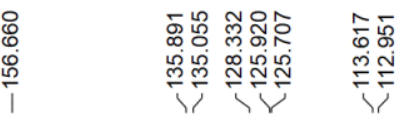

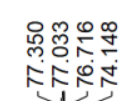

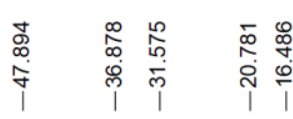

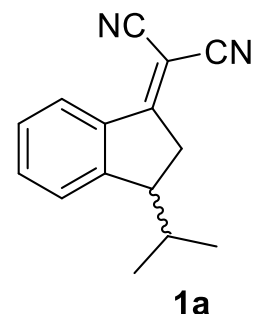

${ }^{13} \mathrm{C}$ NMR $\left(100 \mathrm{MHz}, \mathrm{CDCl}_{3}\right)$
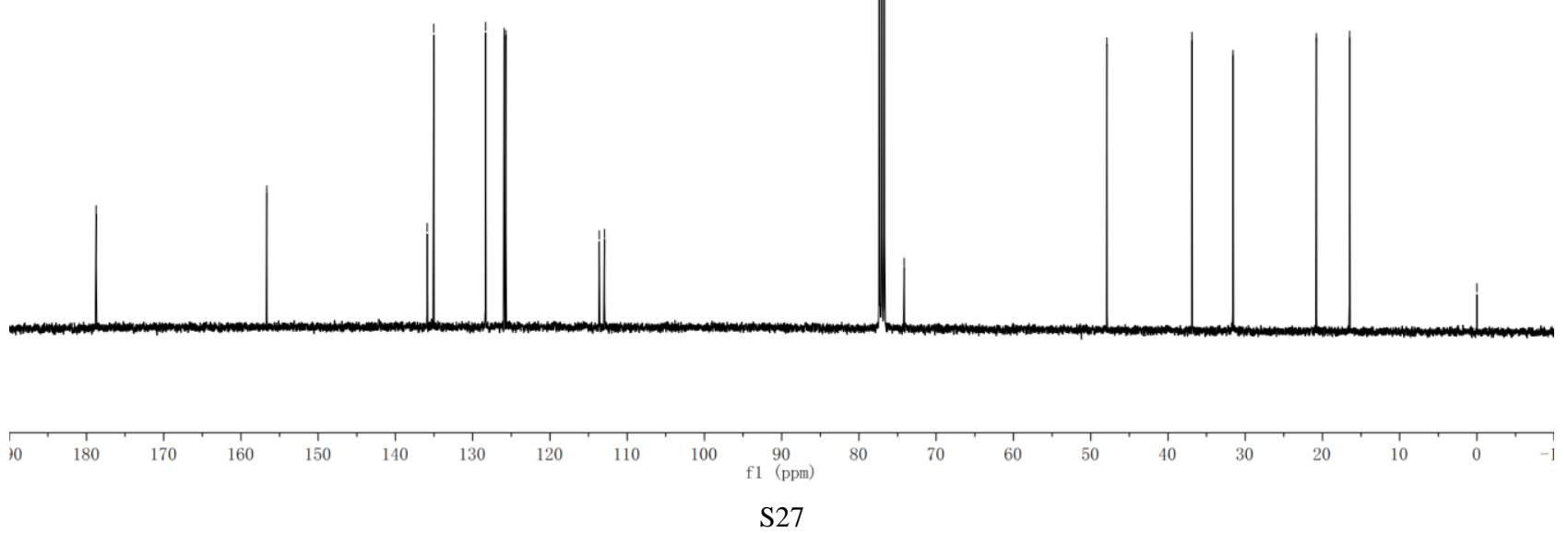


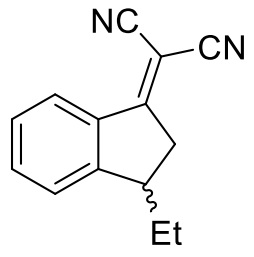

1b

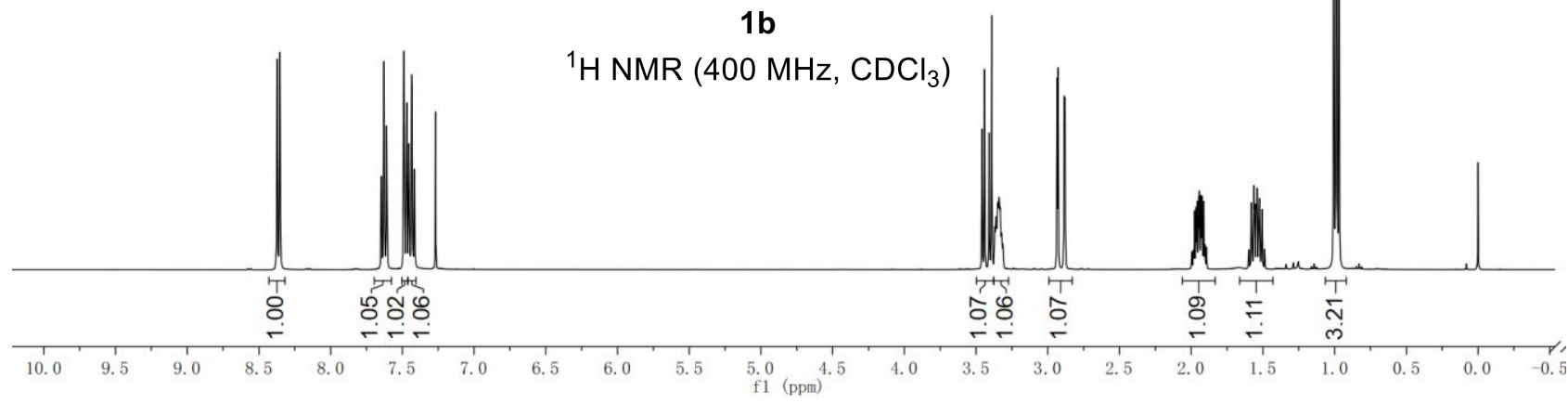

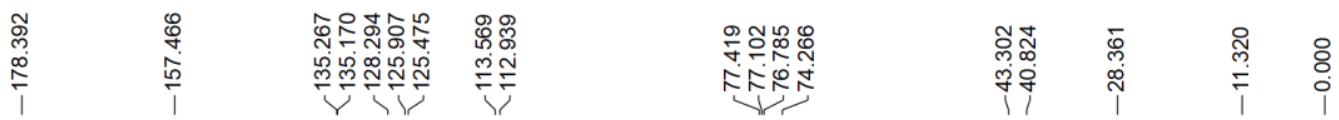

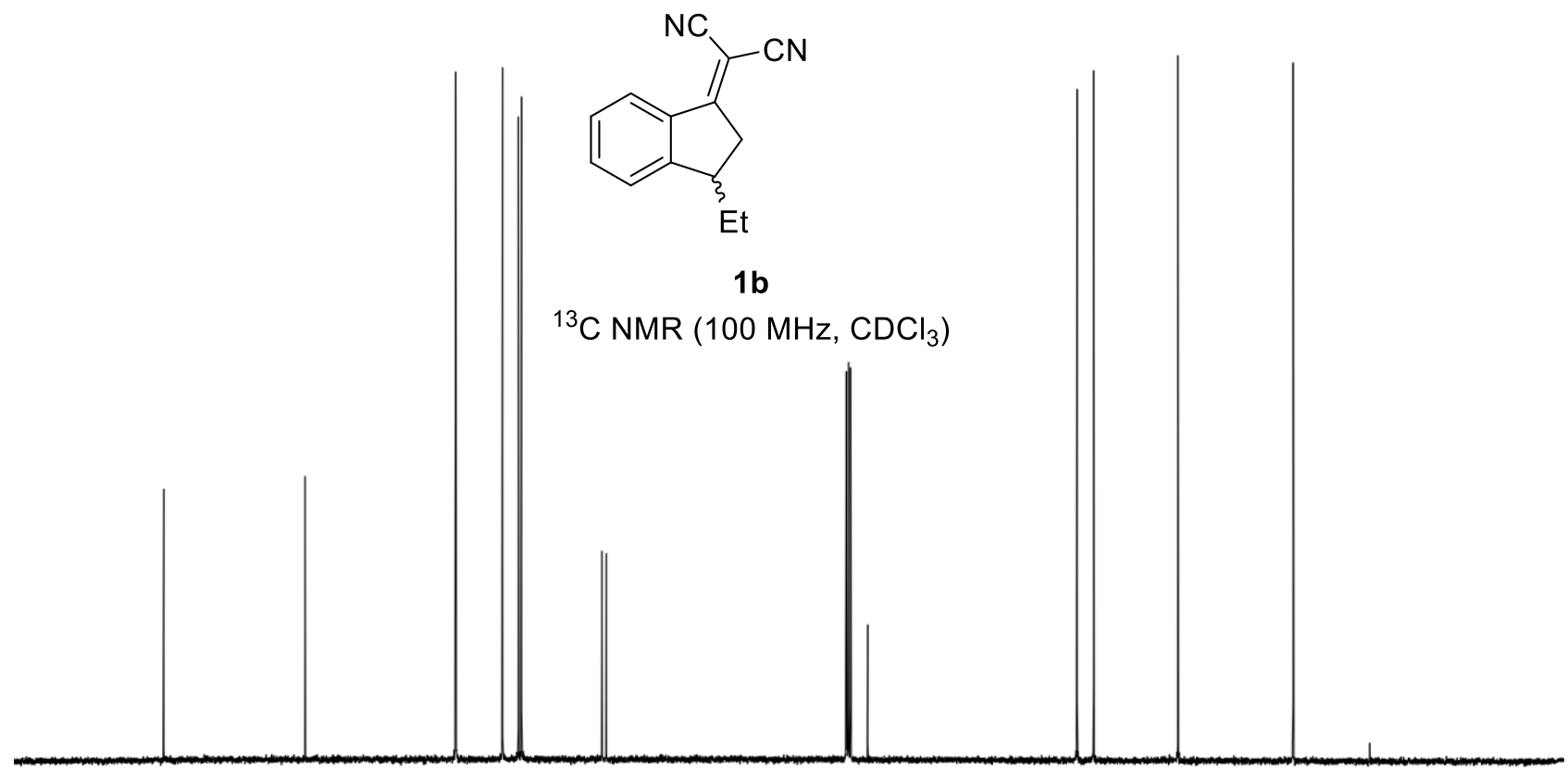

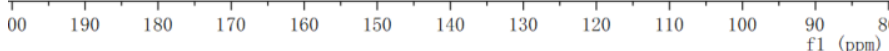



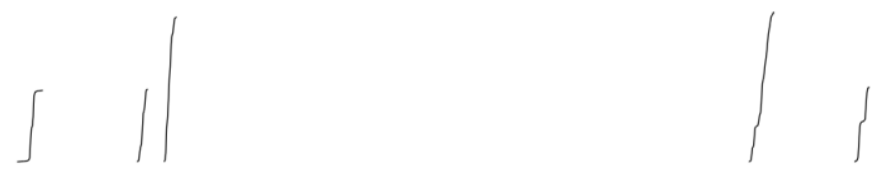<smiles>C[C@H]1CC(=C(C#N)C#N)c2ccccc21</smiles>

$1 \mathrm{c}$

${ }^{1} \mathrm{H}$ NMR $\left(400 \mathrm{MHz}, \mathrm{CDCl}_{3}\right)$

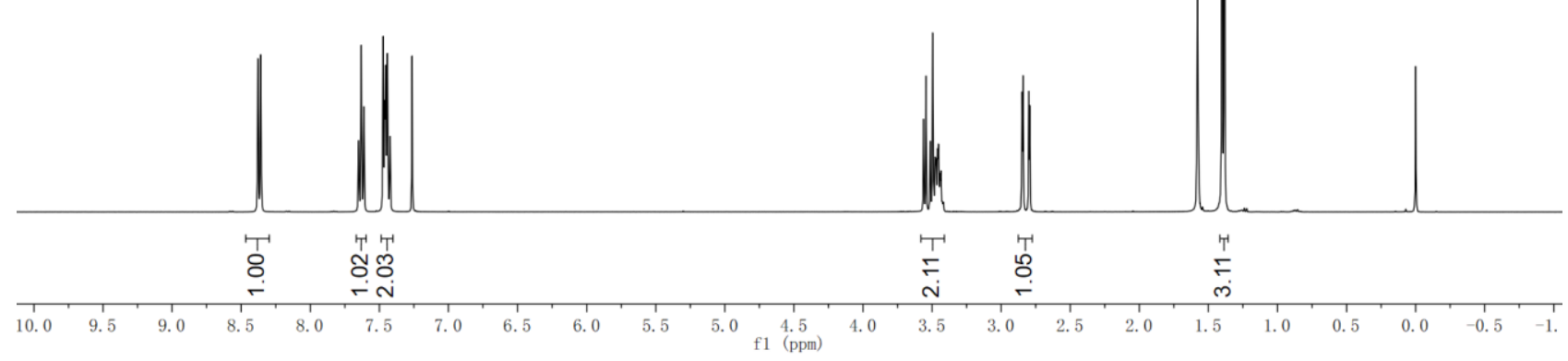<smiles>C[C@H]1CC(=C(C#N)C#N)c2ccccc21</smiles>

$1 \mathrm{c}$

${ }^{13} \mathrm{C}$ NMR $\left(100 \mathrm{MHz}, \mathrm{CDCl}_{3}\right)$ 

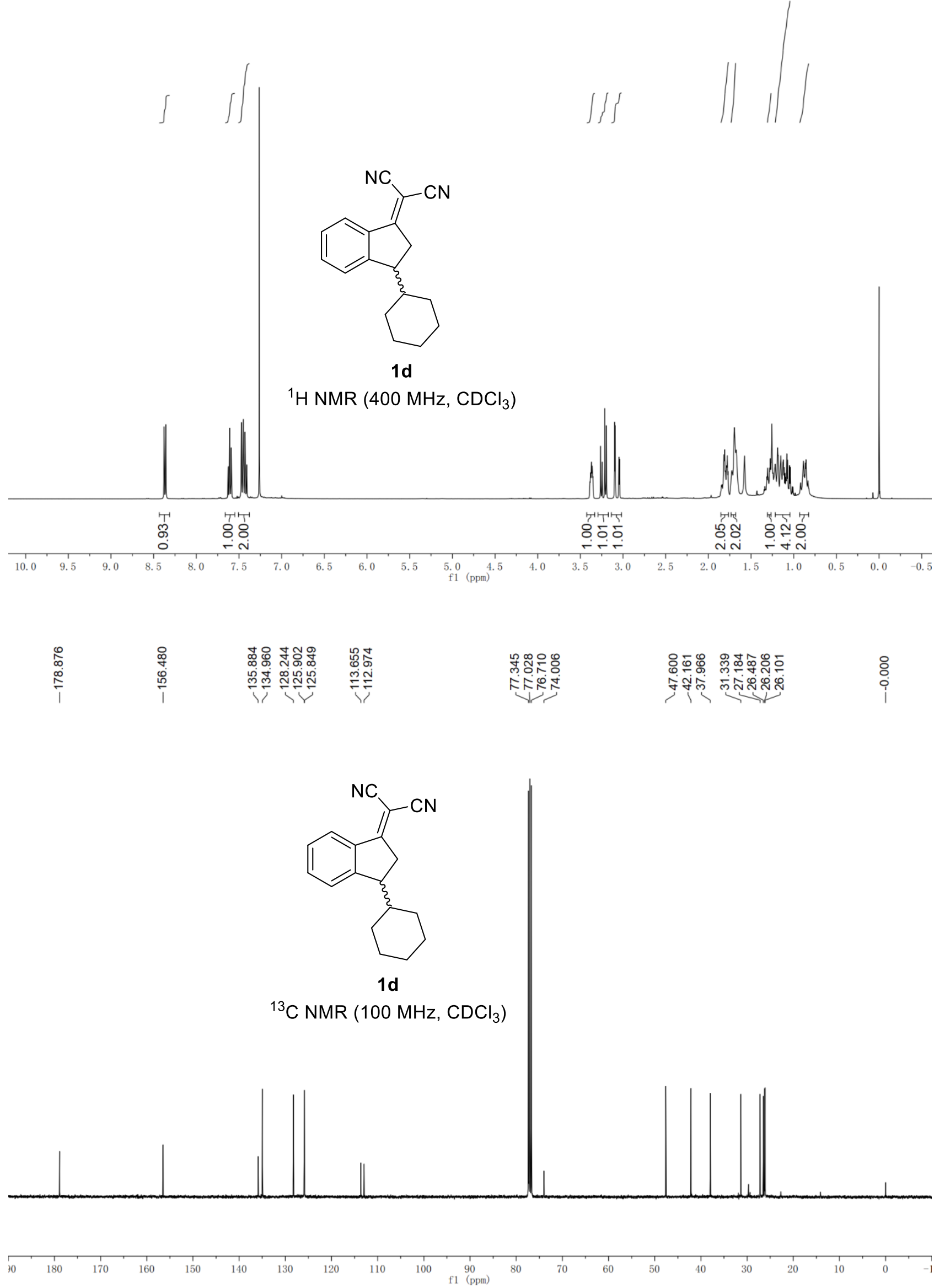


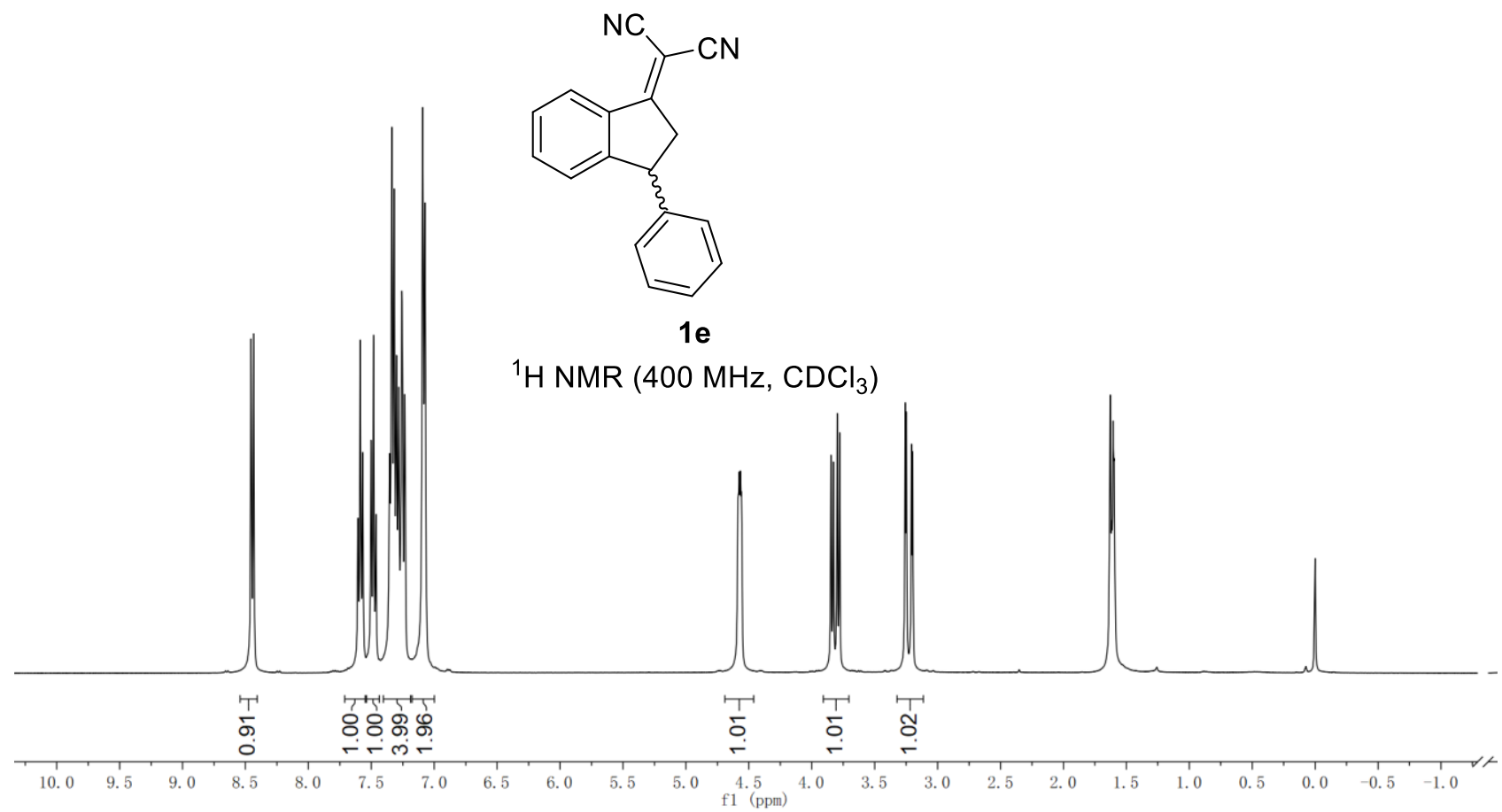

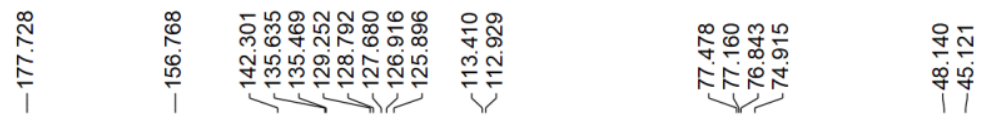

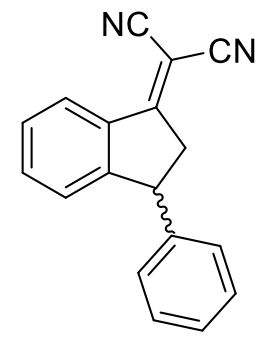

$1 e$

${ }^{13} \mathrm{C}$ NMR $\left(100 \mathrm{MHz}, \mathrm{CDCl}_{3}\right)$

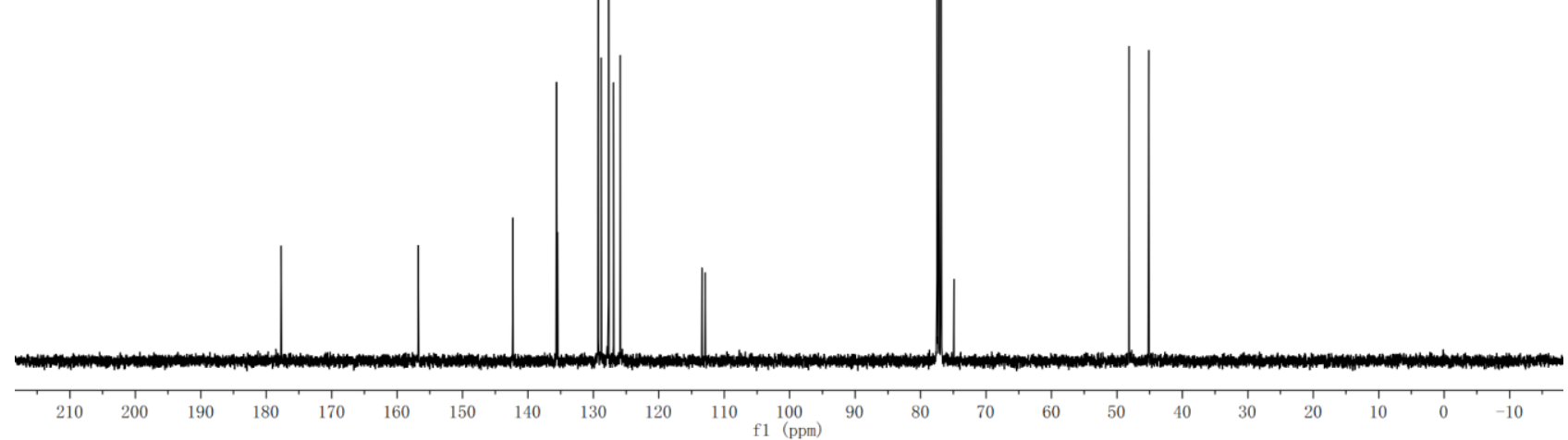


$\int \quad \int$<smiles>CC(C)C1CC(=C(C#N)C#N)c2cc(F)ccc21</smiles>

$\left.\iint\right\}$

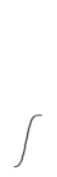

${ }^{1} \mathrm{H}$ NMR $\left(400 \mathrm{MHz}, \mathrm{CDCl}_{3}\right)$

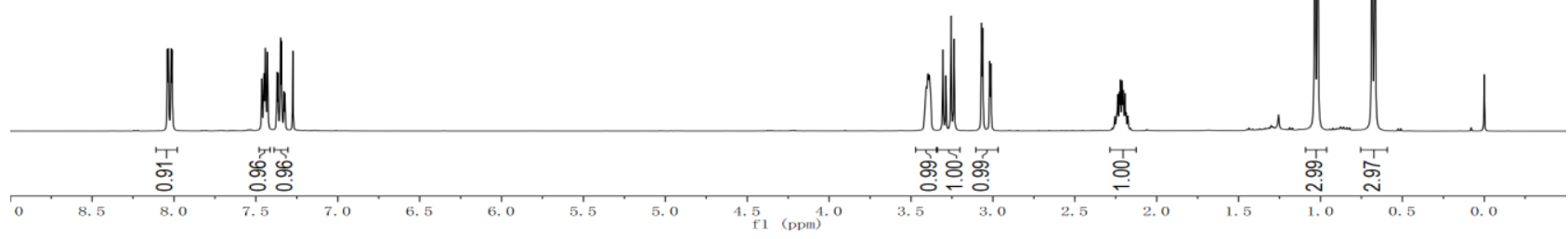

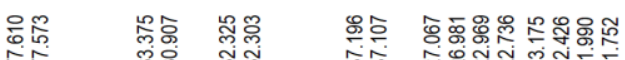

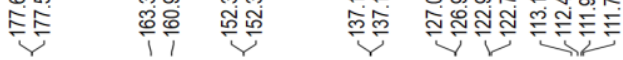

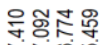

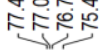

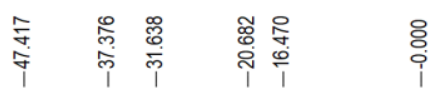<smiles>CC(C)C1CC(=C(C#N)C#N)c2cc(F)ccc21</smiles>

${ }^{13} \mathrm{C}$ NMR $\left(100 \mathrm{MHz}, \mathrm{CDCl}_{3}\right)$
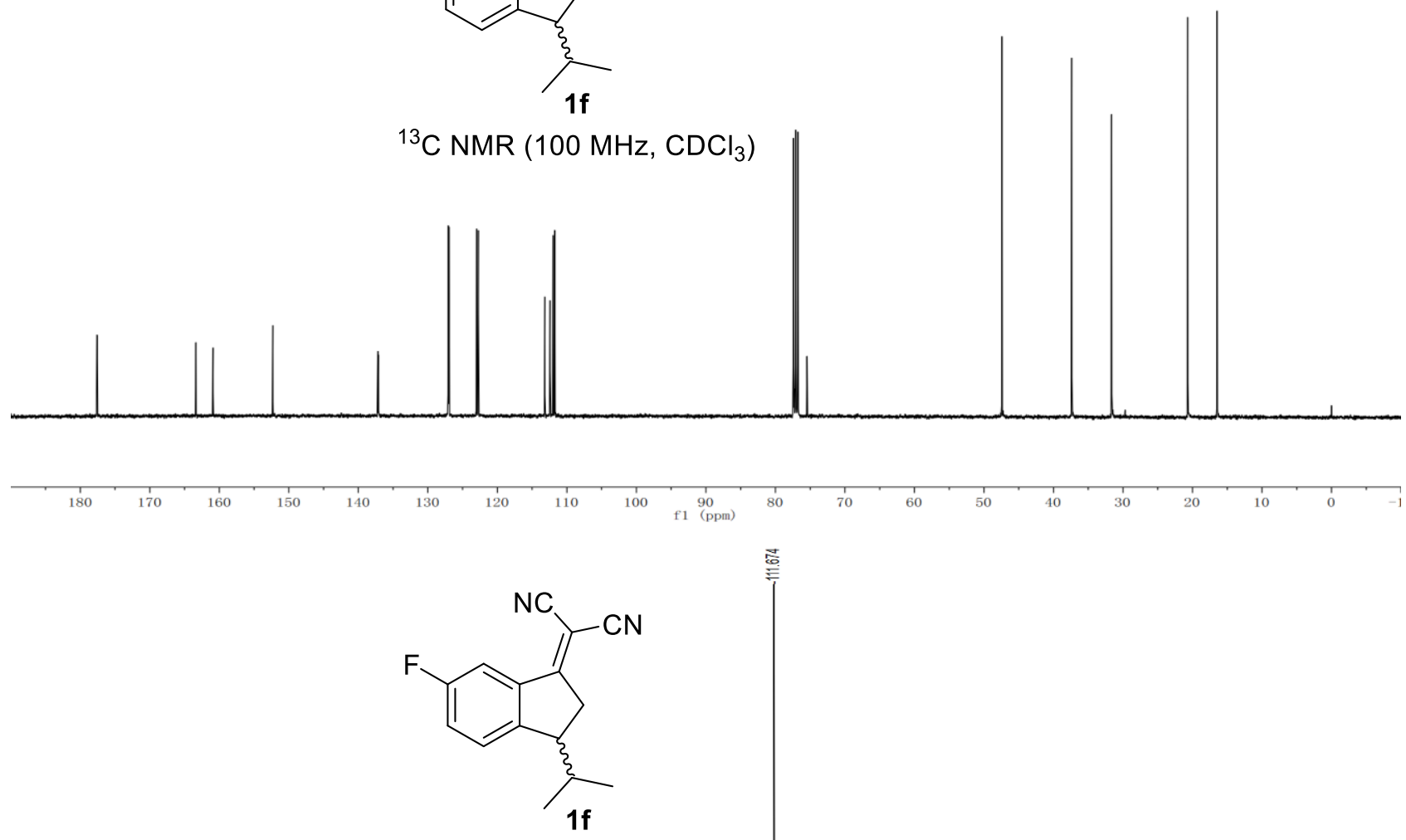

${ }^{19} \mathrm{~F}$ NMR (376 MHz, $\left.\mathrm{CDCl}_{3}\right)$ 


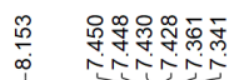

$\iiint$<smiles>Cc1ccc2c(c1)C(=C(C#N)C#N)C[C@H]2C(C)C</smiles>

$1 \mathrm{~g}$

${ }^{1} \mathrm{H}$ NMR $\left(400 \mathrm{MHz}, \mathrm{CDCl}_{3}\right)$

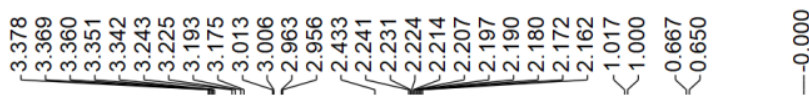

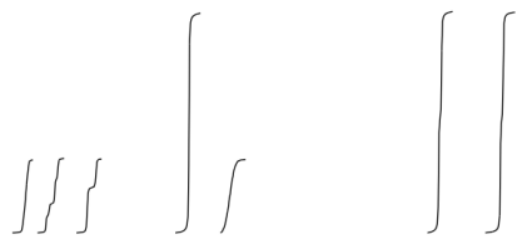

\section{is}

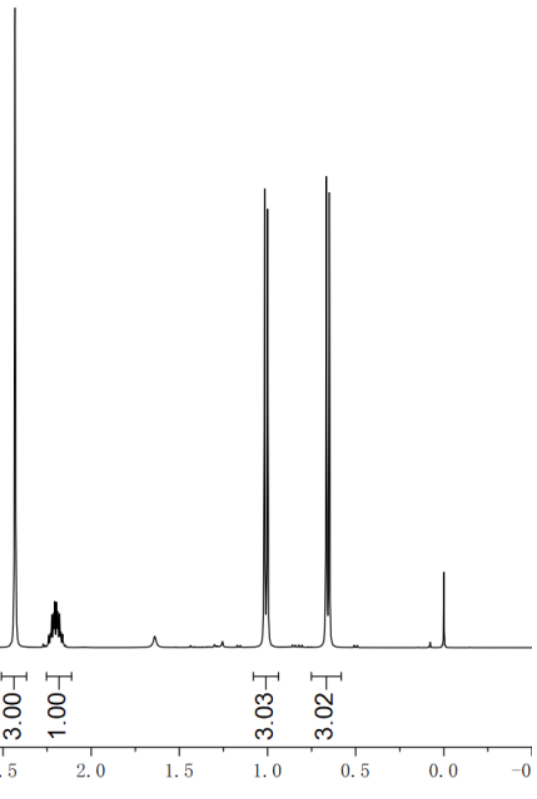

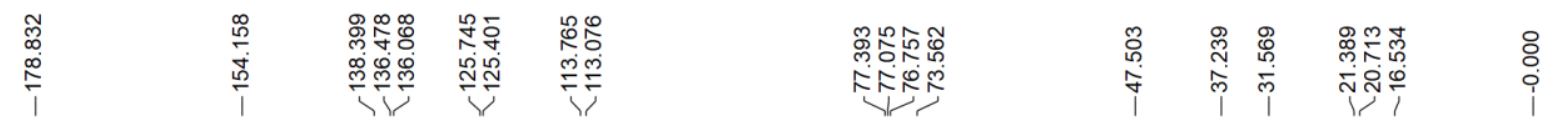<smiles>Cc1ccc2c(c1)C(=C(C#N)C#N)C[C@H]2C(C)C</smiles>

$1 \mathrm{~g}$

${ }^{13} \mathrm{C}$ NMR $\left(100 \mathrm{MHz}, \mathrm{CDCl}_{3}\right)$

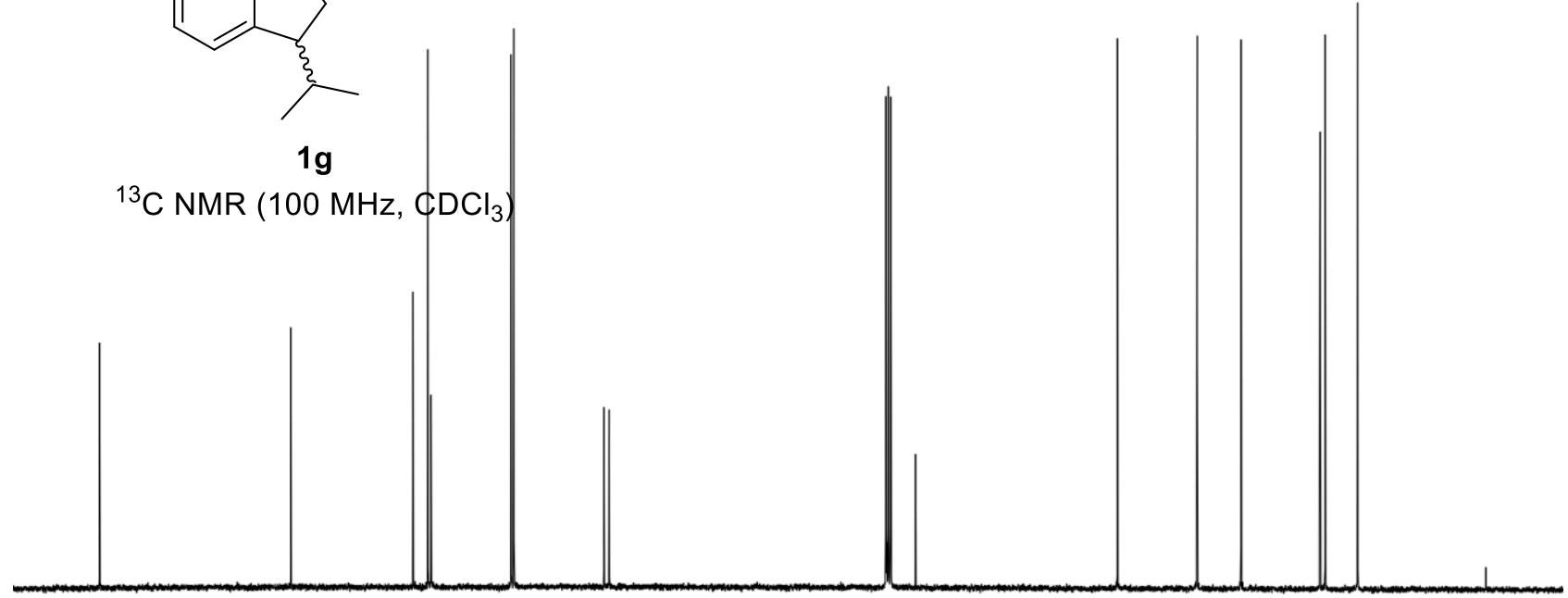

180

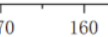




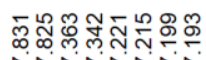

vivivin

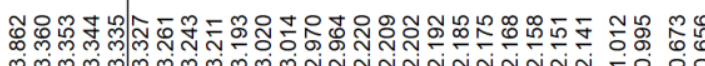

$\underset{\substack{0 \\ i \\ i}}{0}$

$\iint$<smiles>COc1ccc2c(c1)C(=C(C#N)C#N)C[C@H]2C(C)(C)c1ccccc1</smiles>

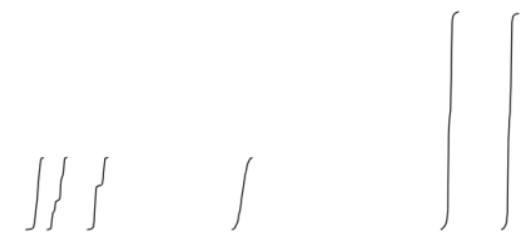

${ }^{1} \mathrm{H}$ NMR $\left(400 \mathrm{MHz}, \mathrm{CDCl}_{3}\right)$

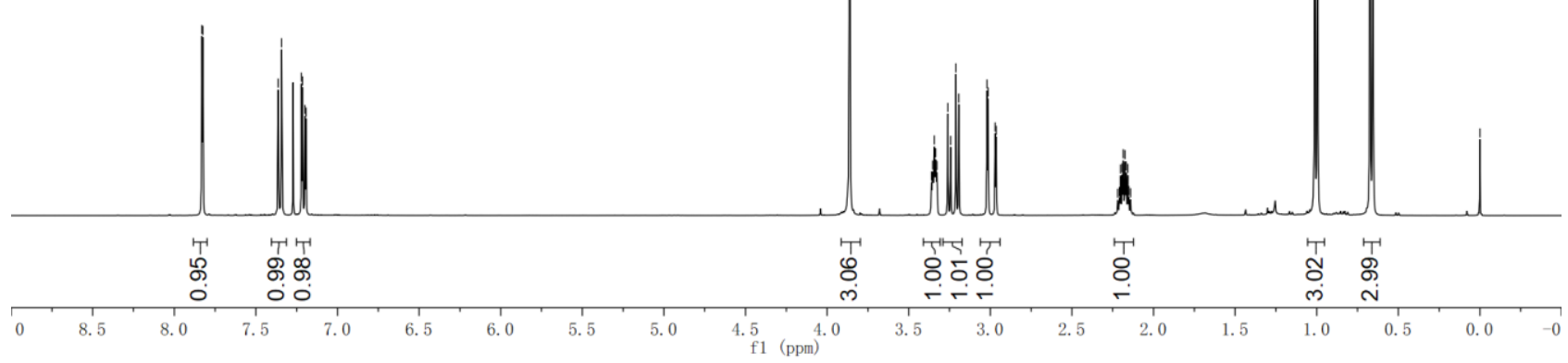

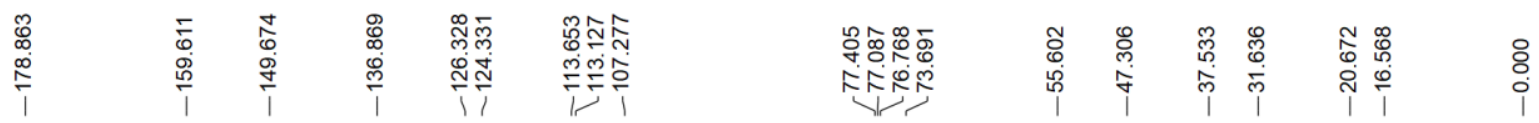<smiles>COc1ccc2c(c1)C(=C(C#N)C#N)CC2C(C)C(C)c1ccccc1</smiles>

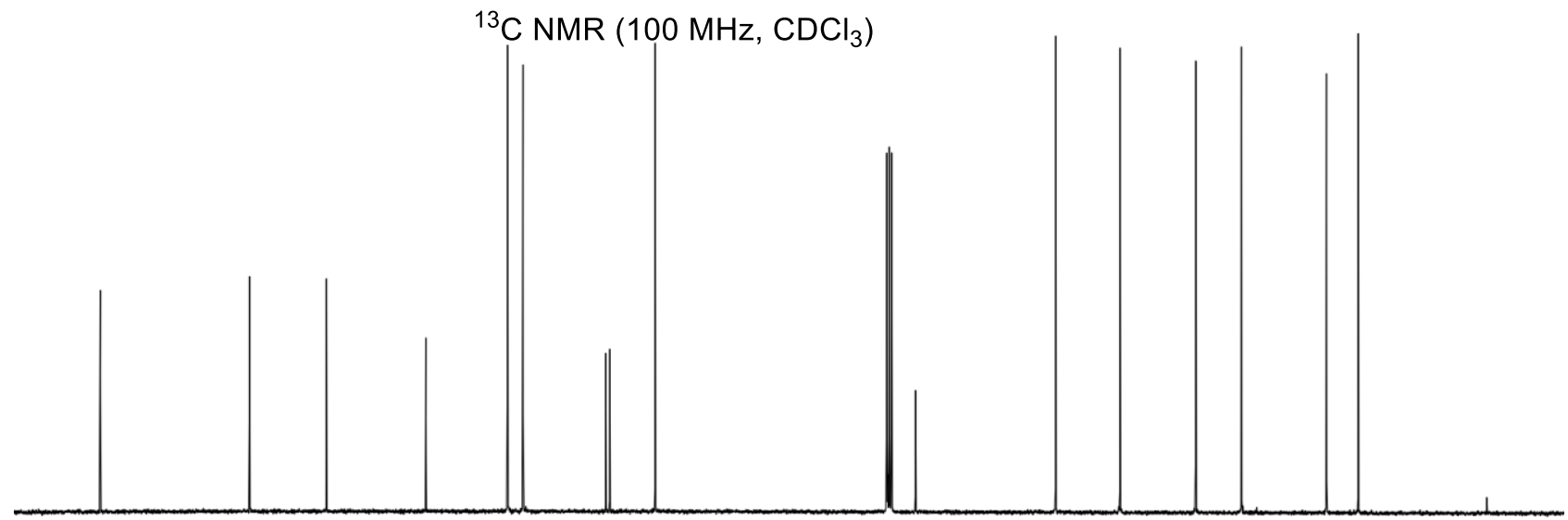

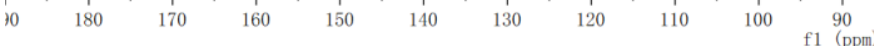




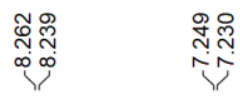
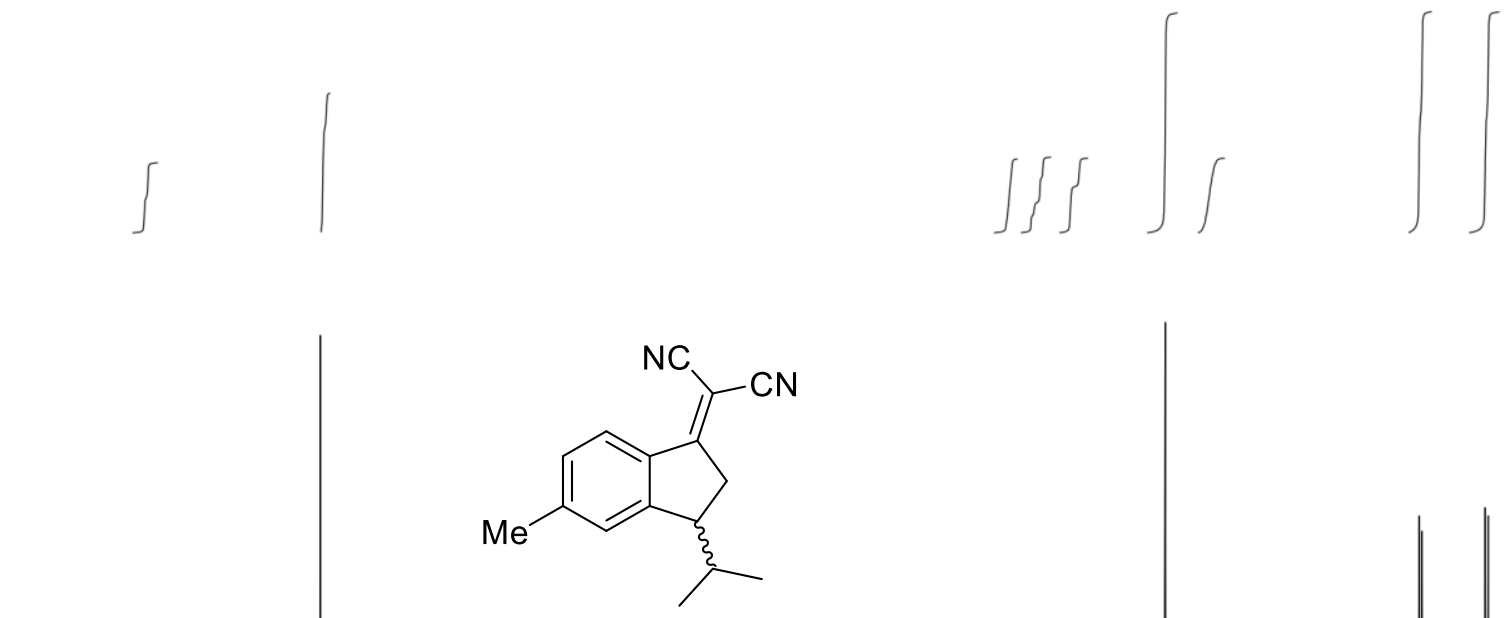

$1 \mathrm{i}$

${ }^{1} \mathrm{H}$ NMR $\left(400 \mathrm{MHz}, \mathrm{CDCl}_{3}\right)$

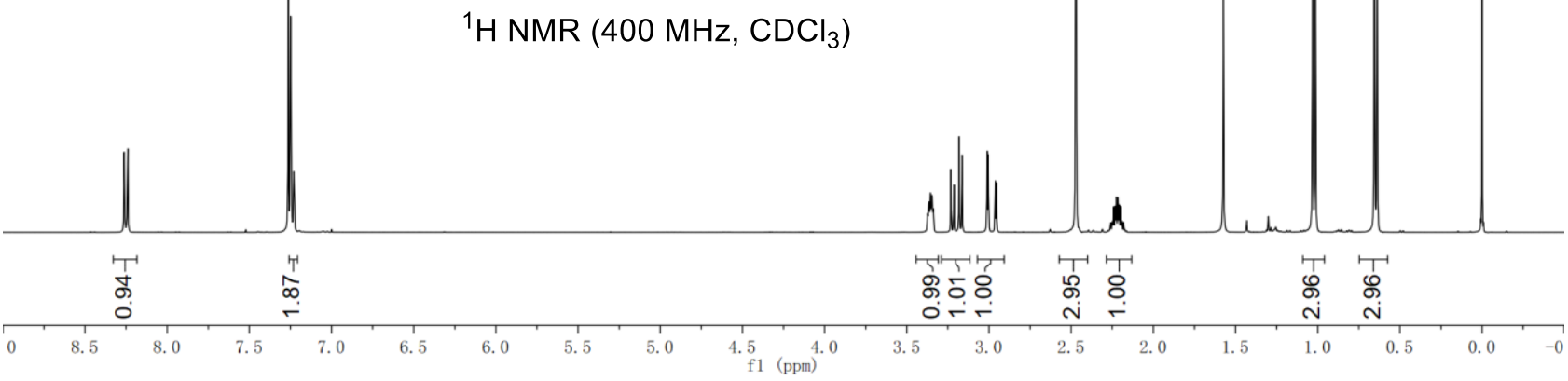

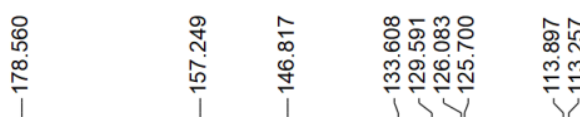

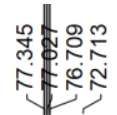

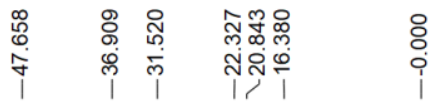<smiles>Cc1ccc2c(c1)C(C(C)C)CC2=C(C#N)C#N</smiles>

$1 \mathrm{i}$

${ }^{13} \mathrm{C} \mathrm{NMR}\left(100 \mathrm{MHz}, \mathrm{CDCl}_{3}\right)$

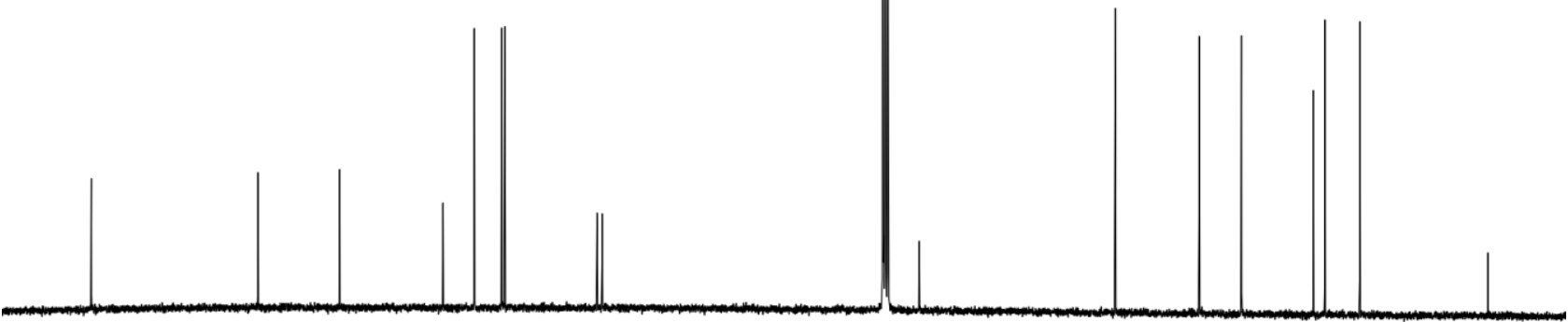

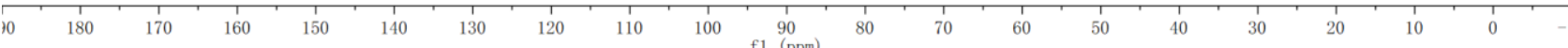



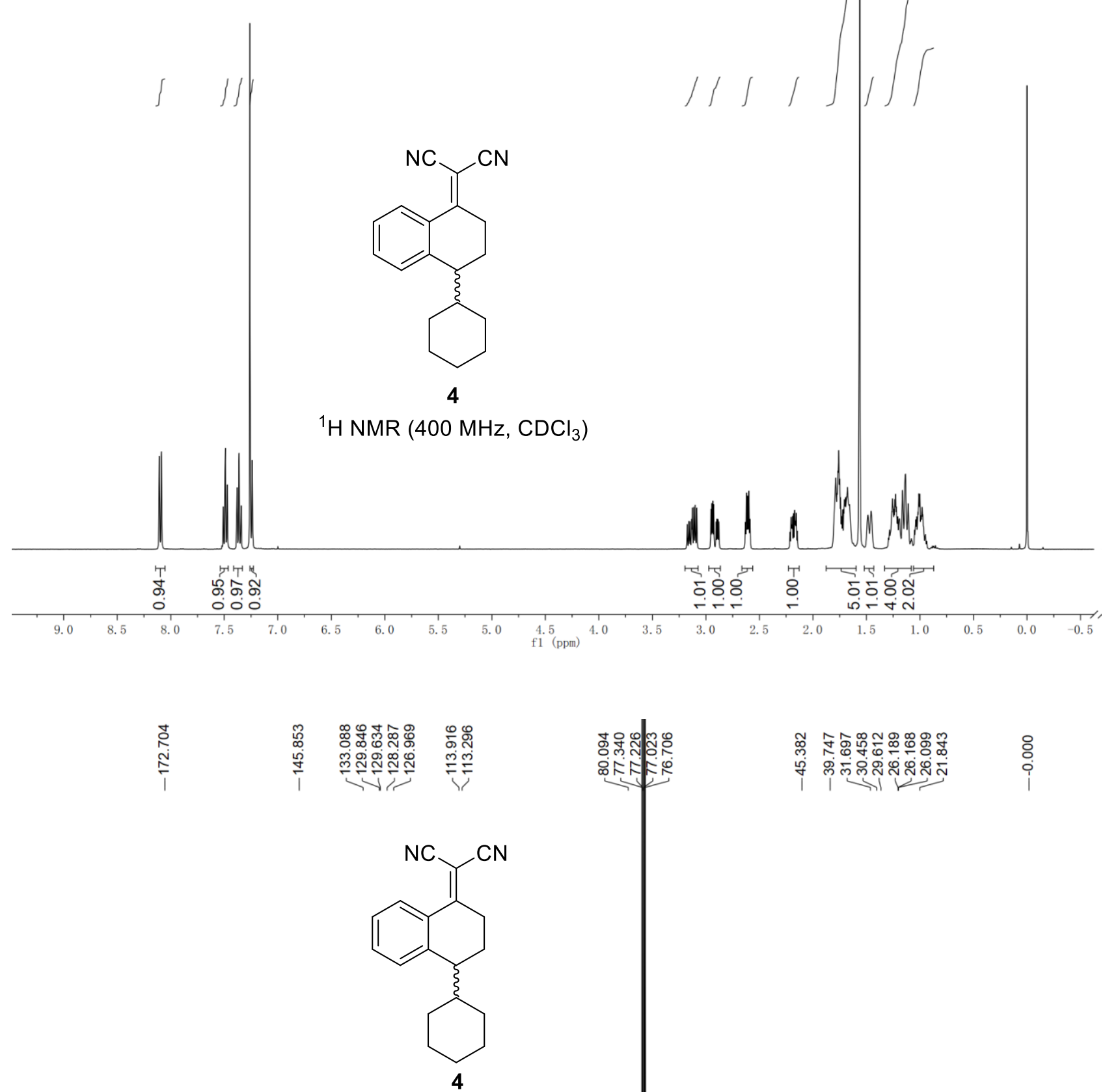

${ }^{13} \mathrm{C}$ NMR $\left(100 \mathrm{MHz}, \mathrm{CDCl}_{3}\right)$
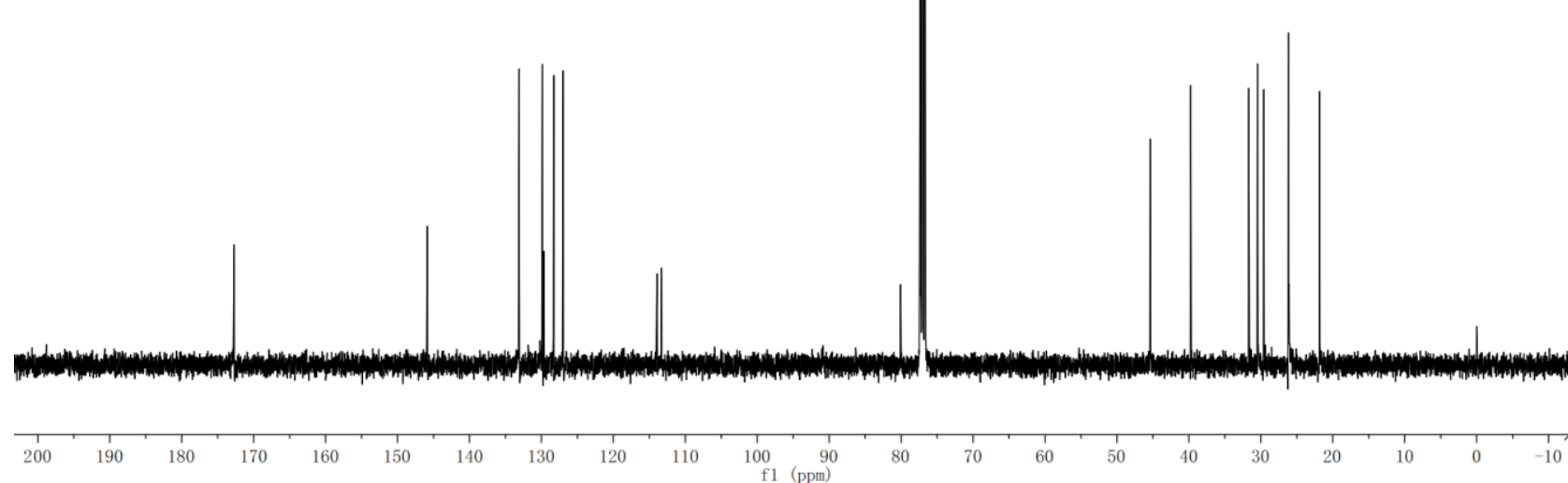

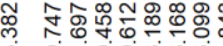

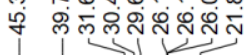

웅 

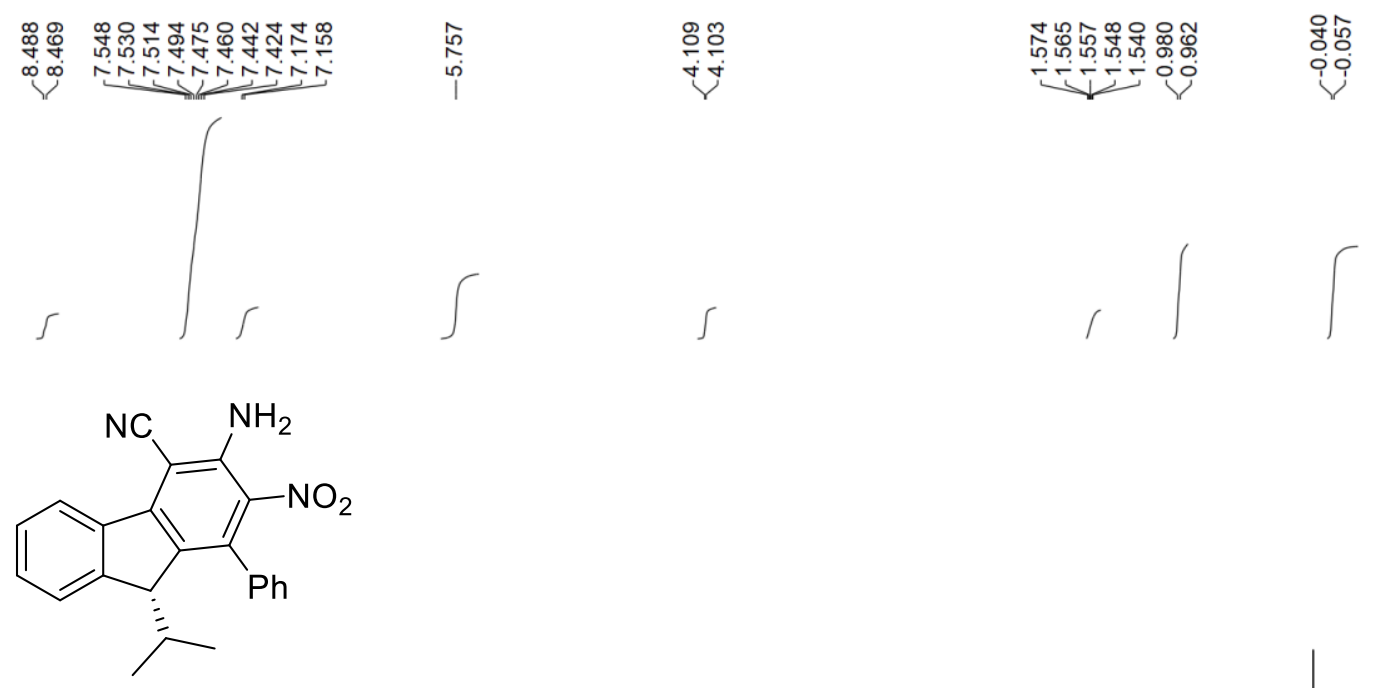

$3 a$

${ }^{1} \mathrm{H}$ NMR (400 MHz, $\mathrm{CDCl}_{3}$ )

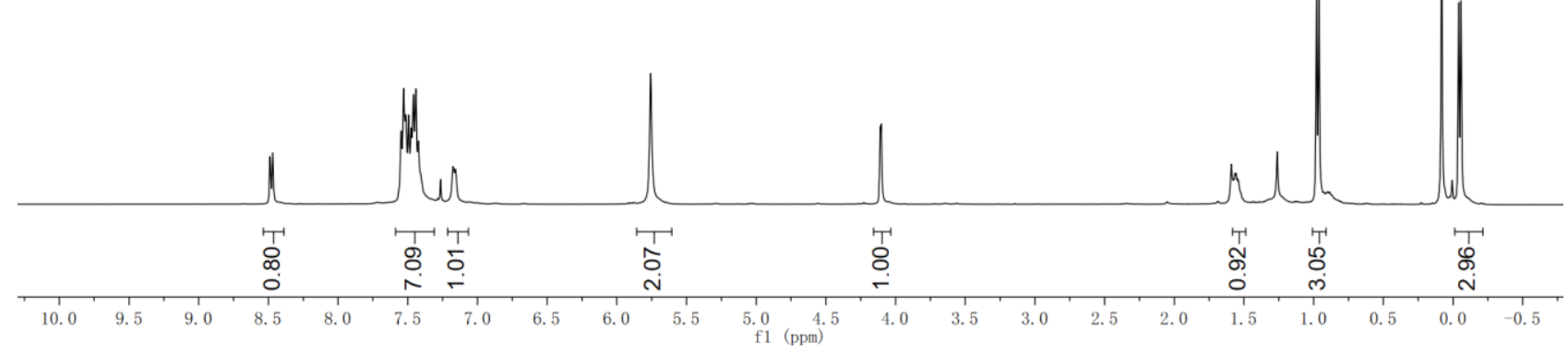

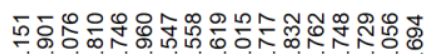

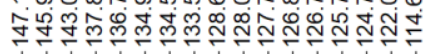

\begin{tabular}{|c|c|c|c|c|}
\hline 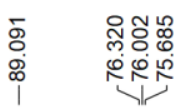 & 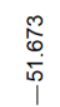 & 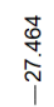 & ల్ల & $\begin{array}{l}\stackrel{\rho}{\stackrel{\rho}{\rho}} \\
\stackrel{\rho}{\rho}\end{array}$ \\
\hline
\end{tabular}

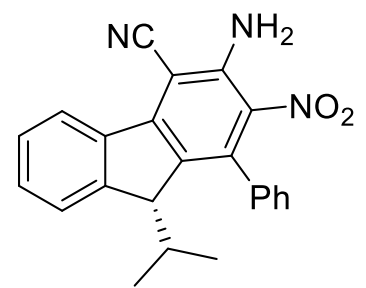

$3 a$

${ }^{13} \mathrm{C}$ NMR $\left(100 \mathrm{MHz}, \mathrm{CDCl}_{3}\right)$
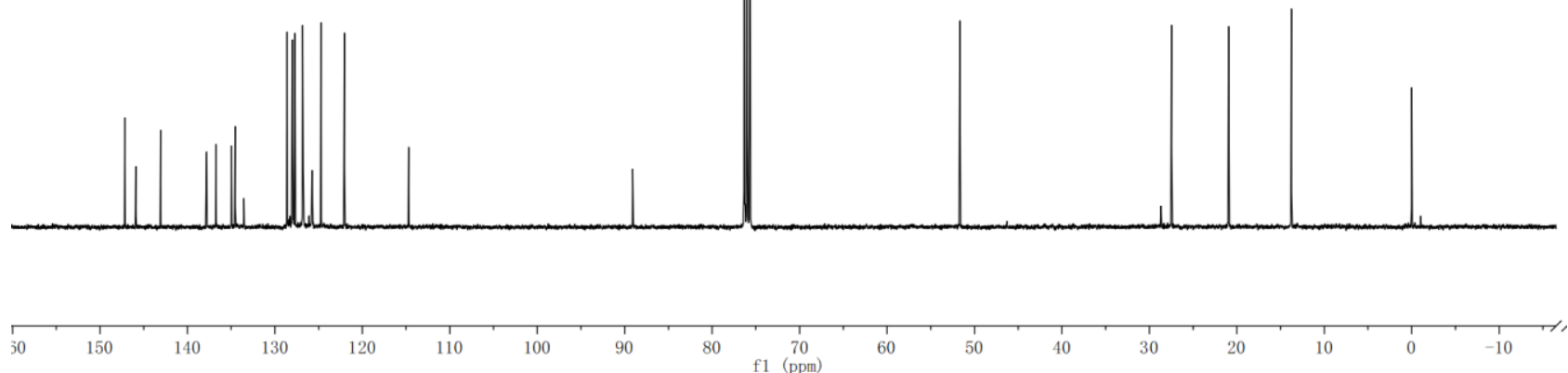


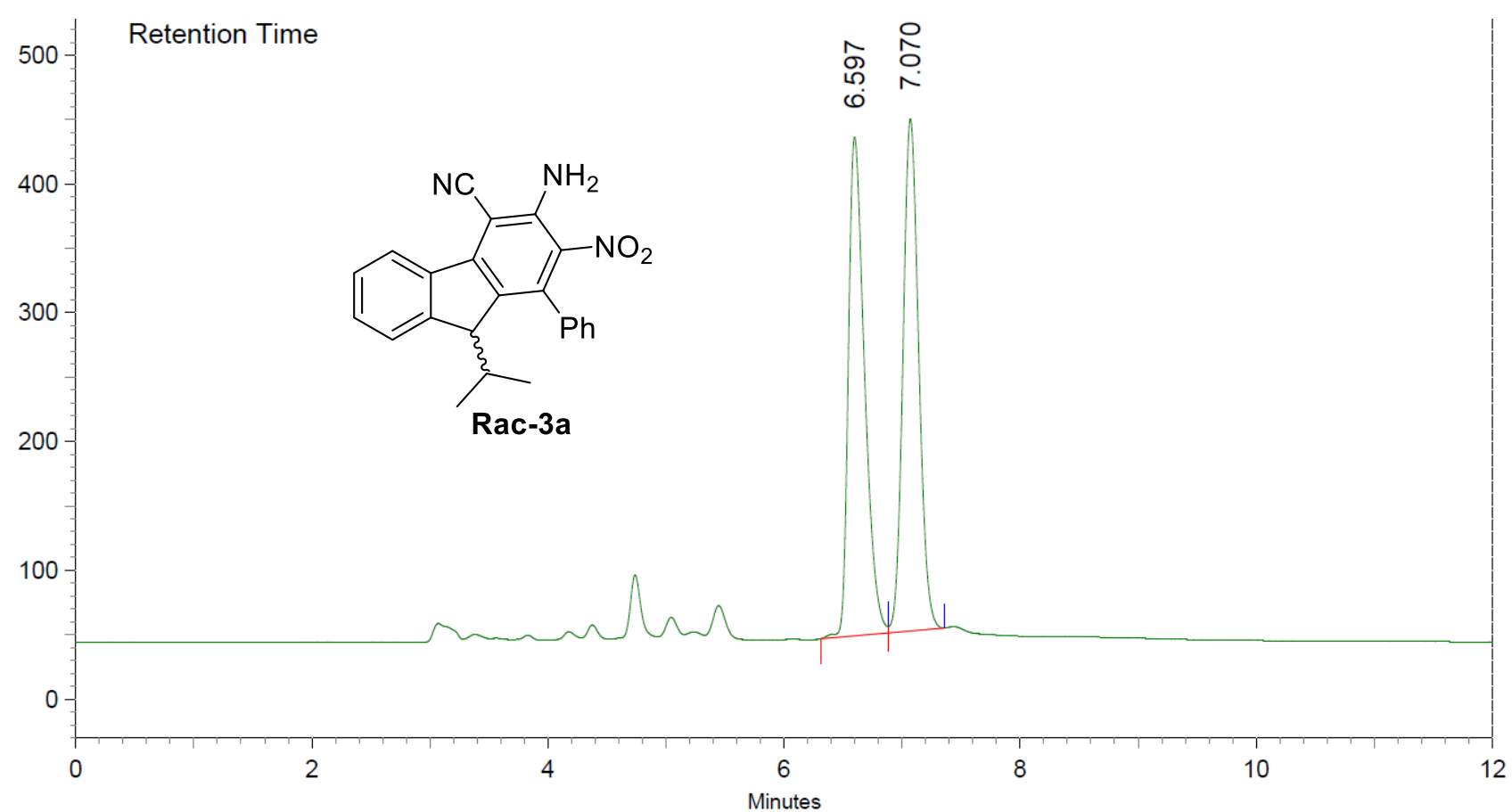

\begin{tabular}{llllll} 
Peak No. & Ret Time & Width & Height & Area & Area [\%] \\
\hline 1 & 6.597 & 0.570 & 6498969 & 61793675 & 50.0340 \\
2 & 7.070 & 0.473 & 6676205 & 61709631 & 49.9660 \\
\hline \multicolumn{2}{l}{} & & 13175174 & 123503306 & 100.0000 \\
\hline
\end{tabular}

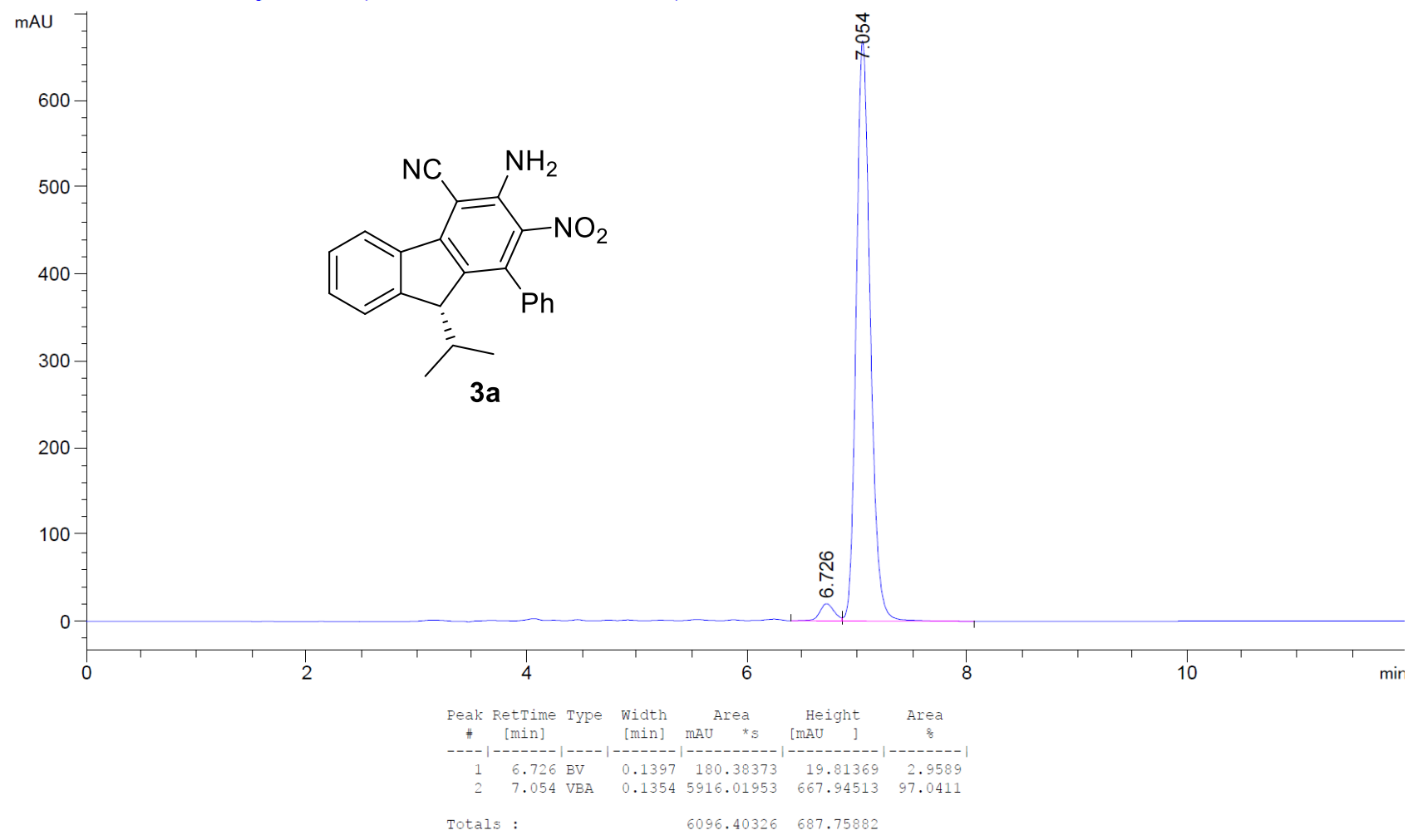




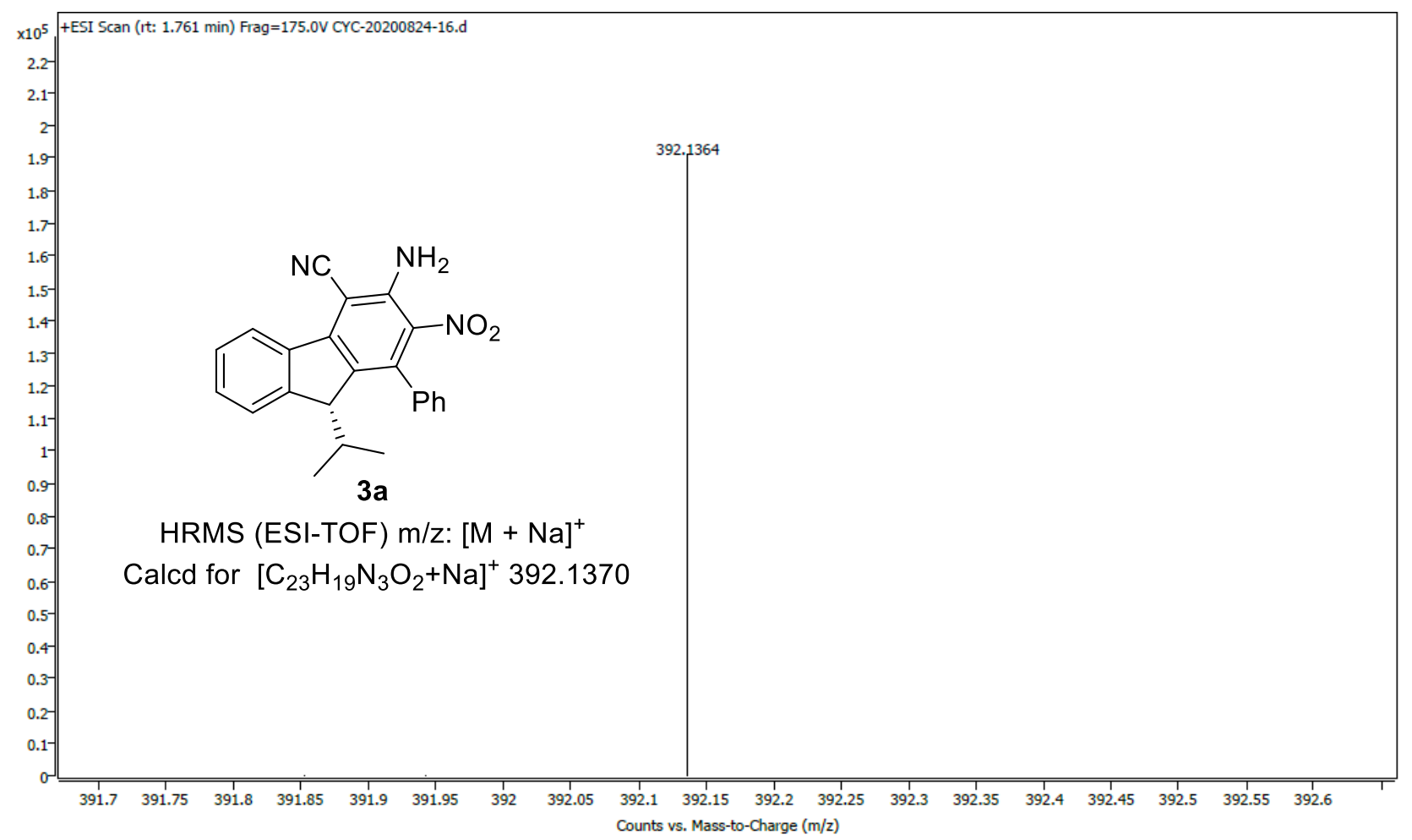



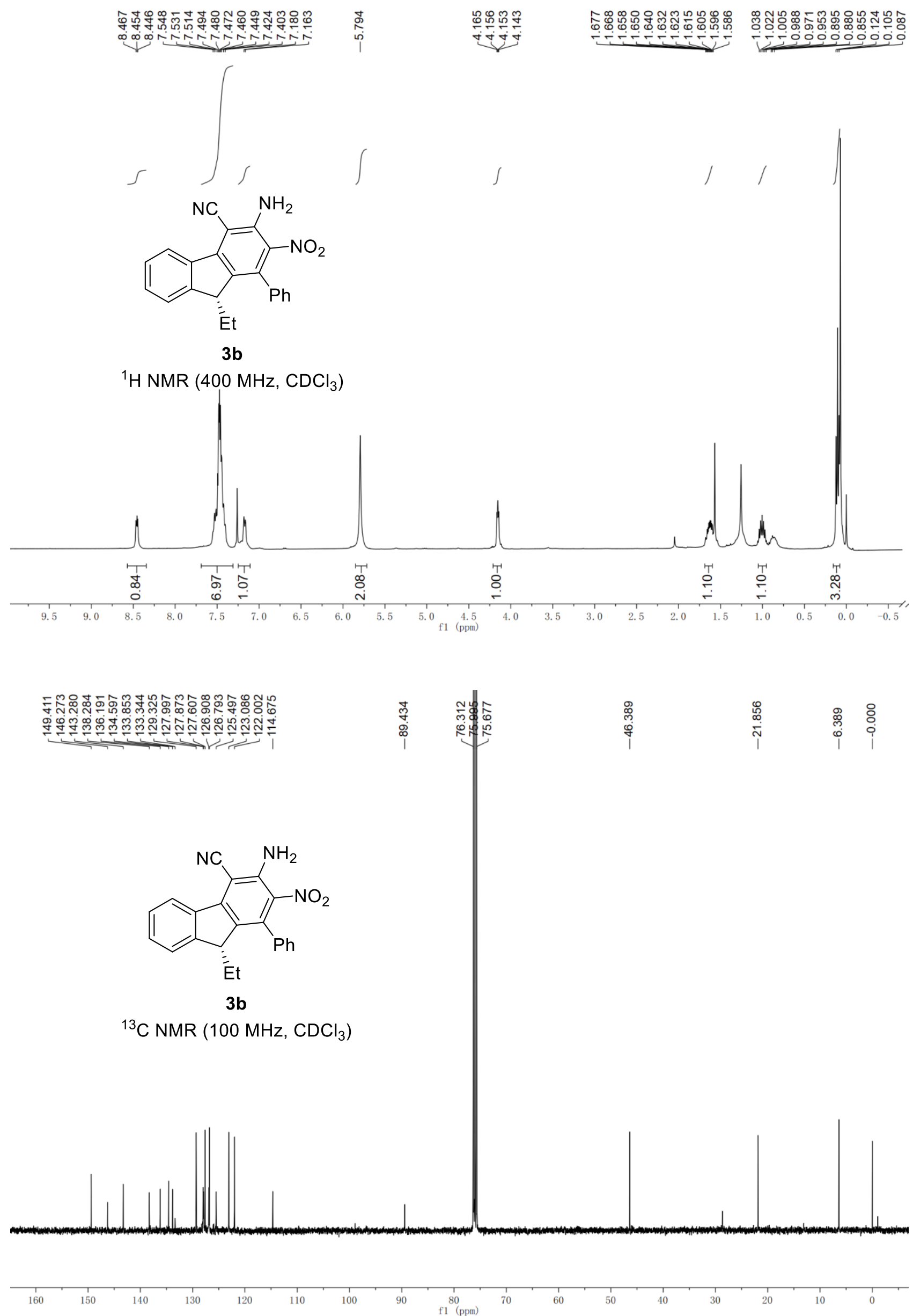

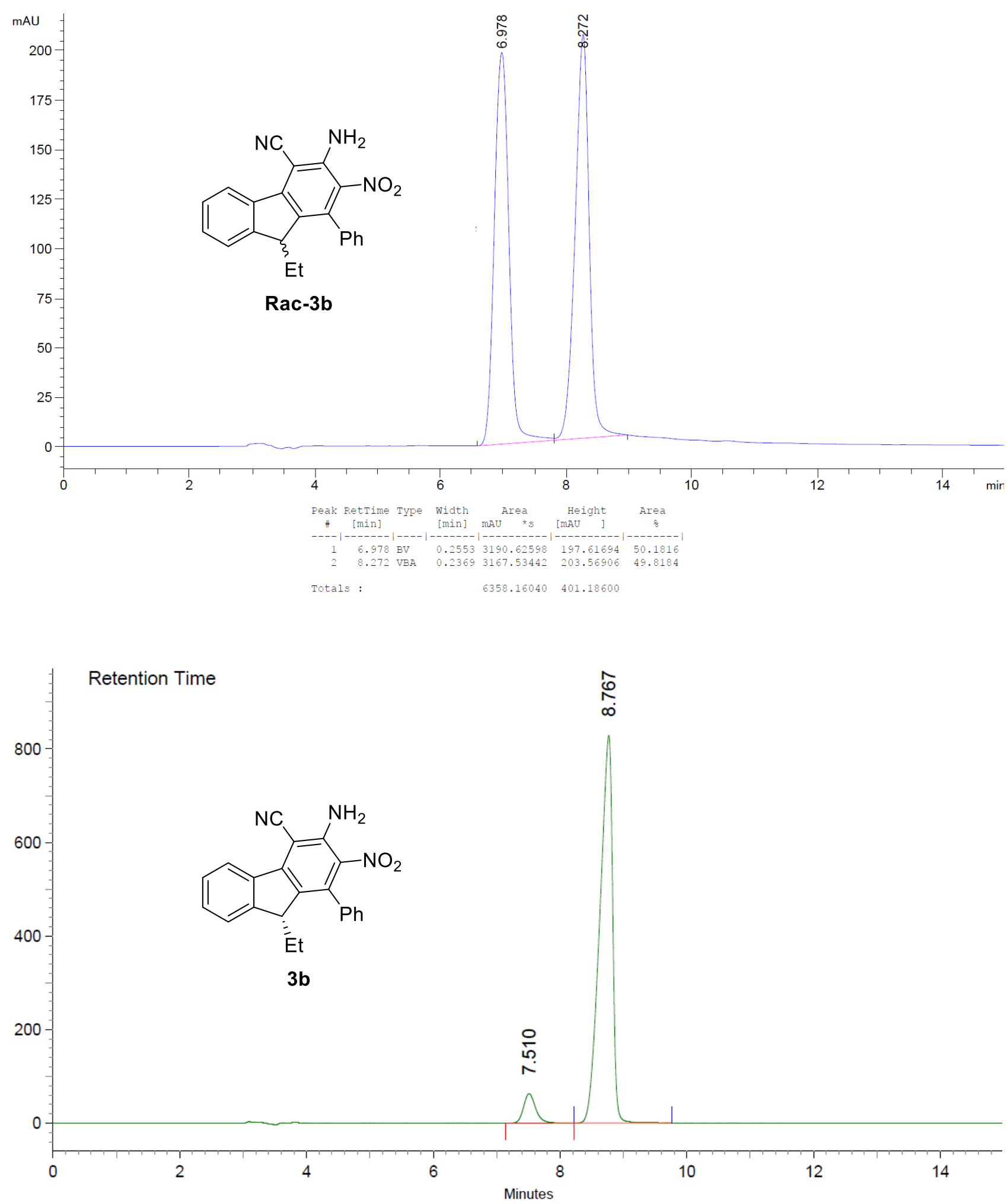

\begin{tabular}{llllll} 
Peak No. & Ret Time & Width & Height & Area & Area [\%] \\
\hline 1 & 7.510 & 1.073 & 1057682 & 14056712 & 6.7351 \\
2 & 8.767 & 1.540 & 13897216 & 194651823 & 93.2649 \\
\hline \multicolumn{2}{l}{ Totals } & & 14954898 & 208708535 & 100.0000 \\
\hline
\end{tabular}




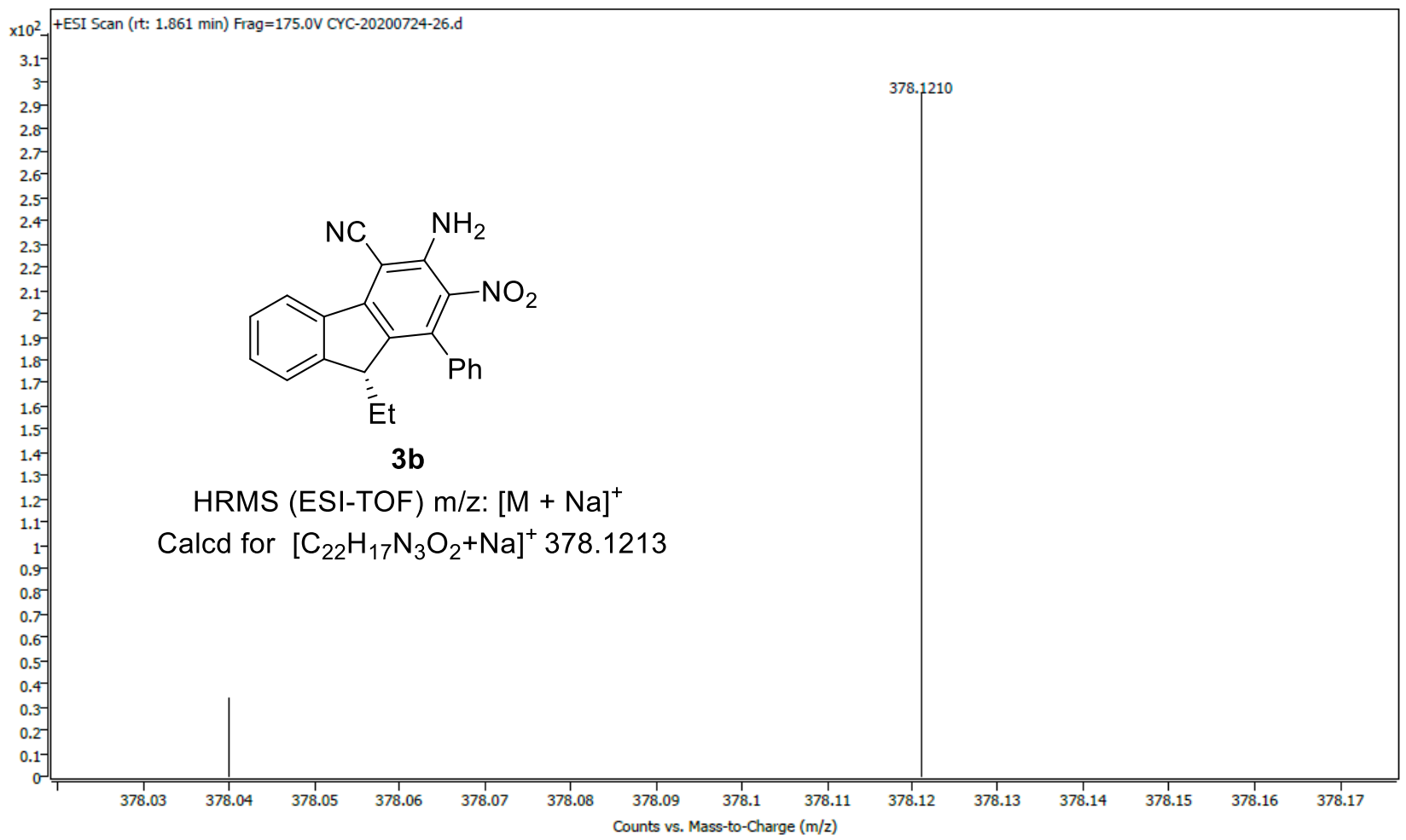




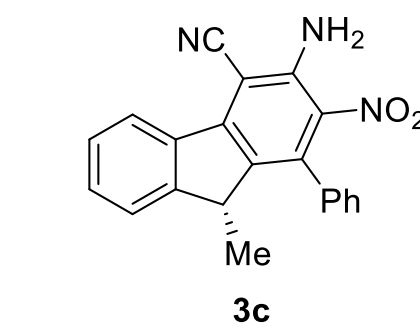

${ }^{1} \mathrm{H}$ NMR $\left(400 \mathrm{MHz}, \mathrm{CDC} \|_{3}\right)$

\section{5}




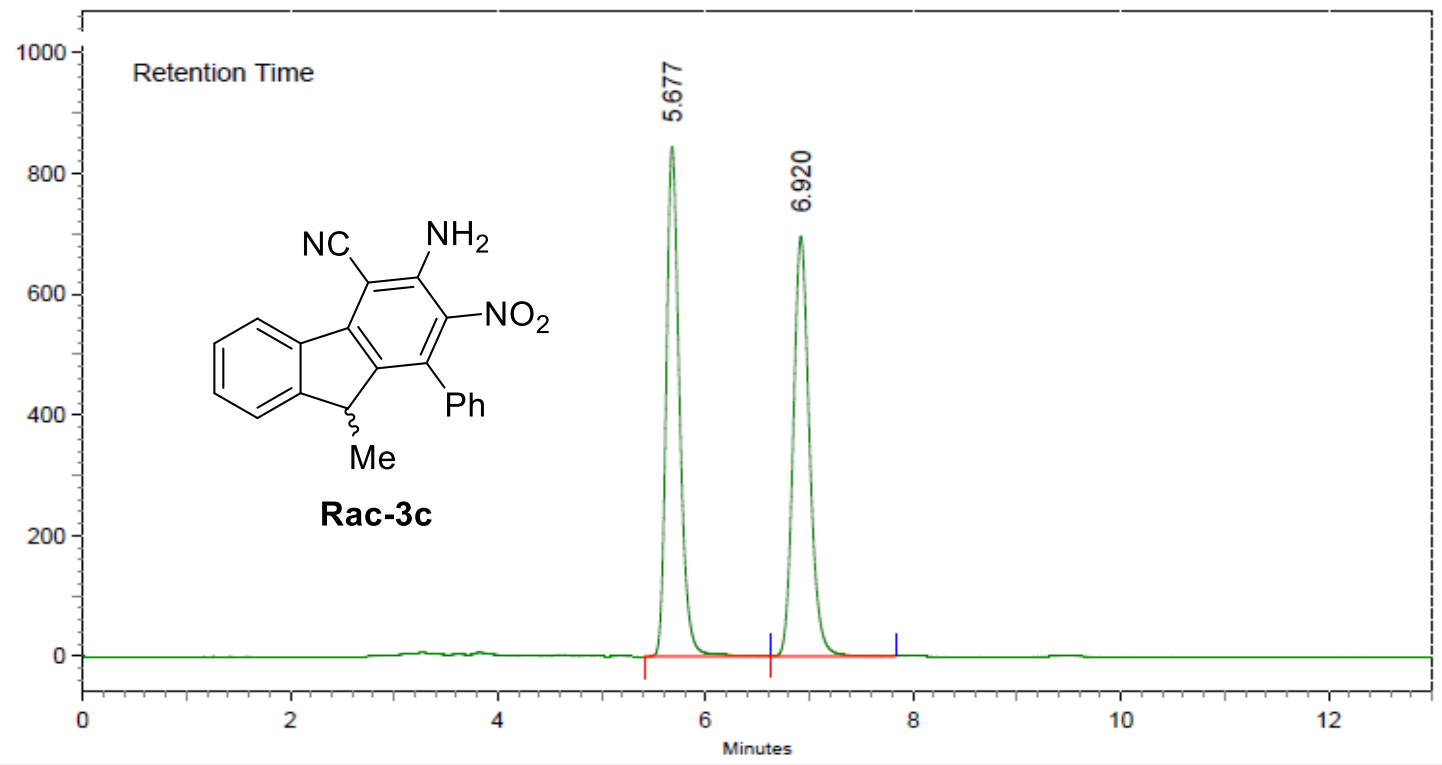

AREA PERCENT REPORT

\begin{tabular}{lllll|l} 
Peak No. & Ret Time & Width & Height & Area & Area [\%] \\
\hline 1 & 5.677 & 1.223 & 14167992 & 126488582 & 50.0136 \\
2 & 6.920 & 1.200 & 11674279 & 126419852 & 49.9864 \\
\hline Totals & & & & \\
\hline
\end{tabular}

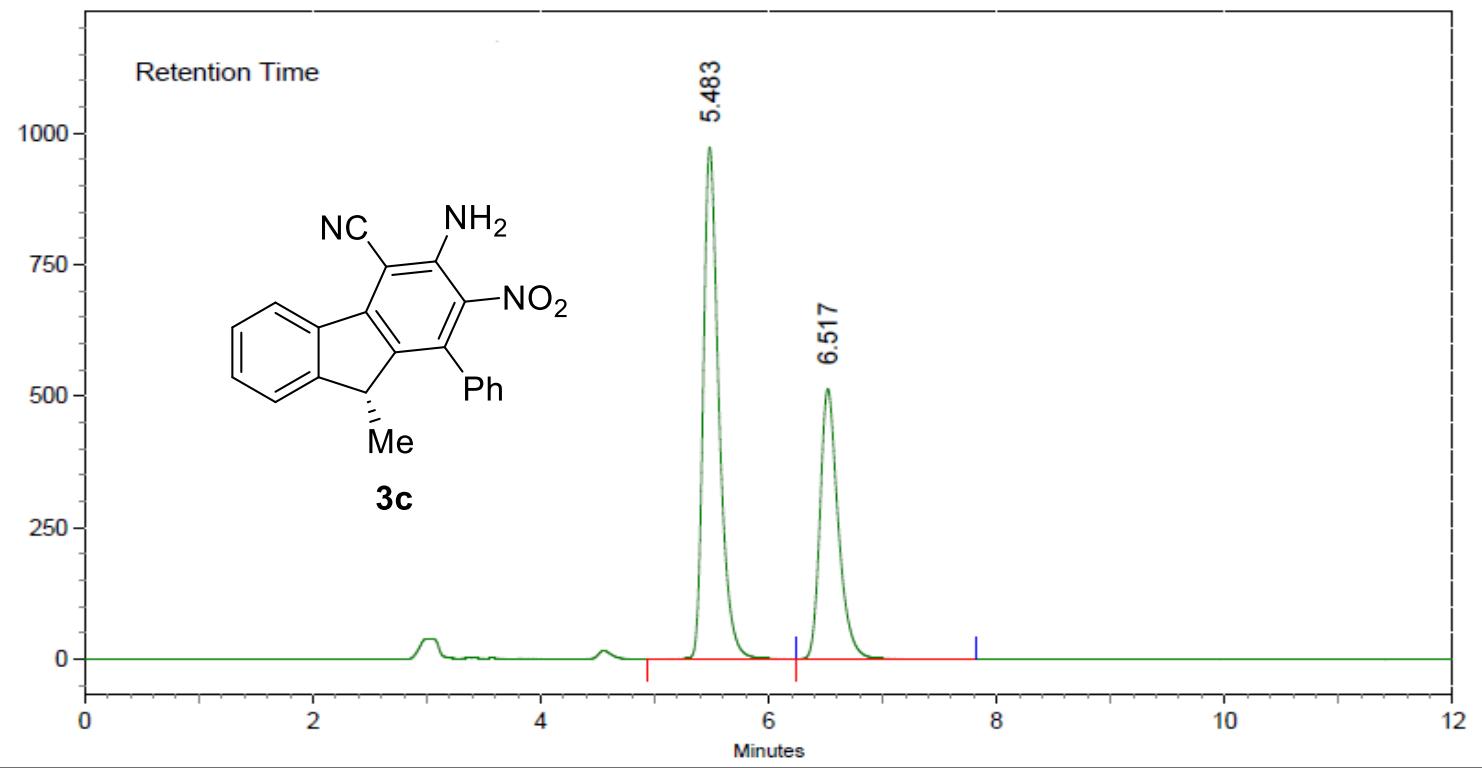

AREA PERCENT REPORT

\begin{tabular}{llllll|} 
Peak No. & Ret Time & Width & Height & Area & Area [\%] \\
\hline 1 & 5.483 & 1.307 & 16316248 & 155508736 & 61.9480 \\
2 & 6.517 & 1.573 & 8615182 & 95522298 & 38.0520 \\
\hline Totals & & 24931430 & 251031034 & 100.0000 \\
\hline
\end{tabular}




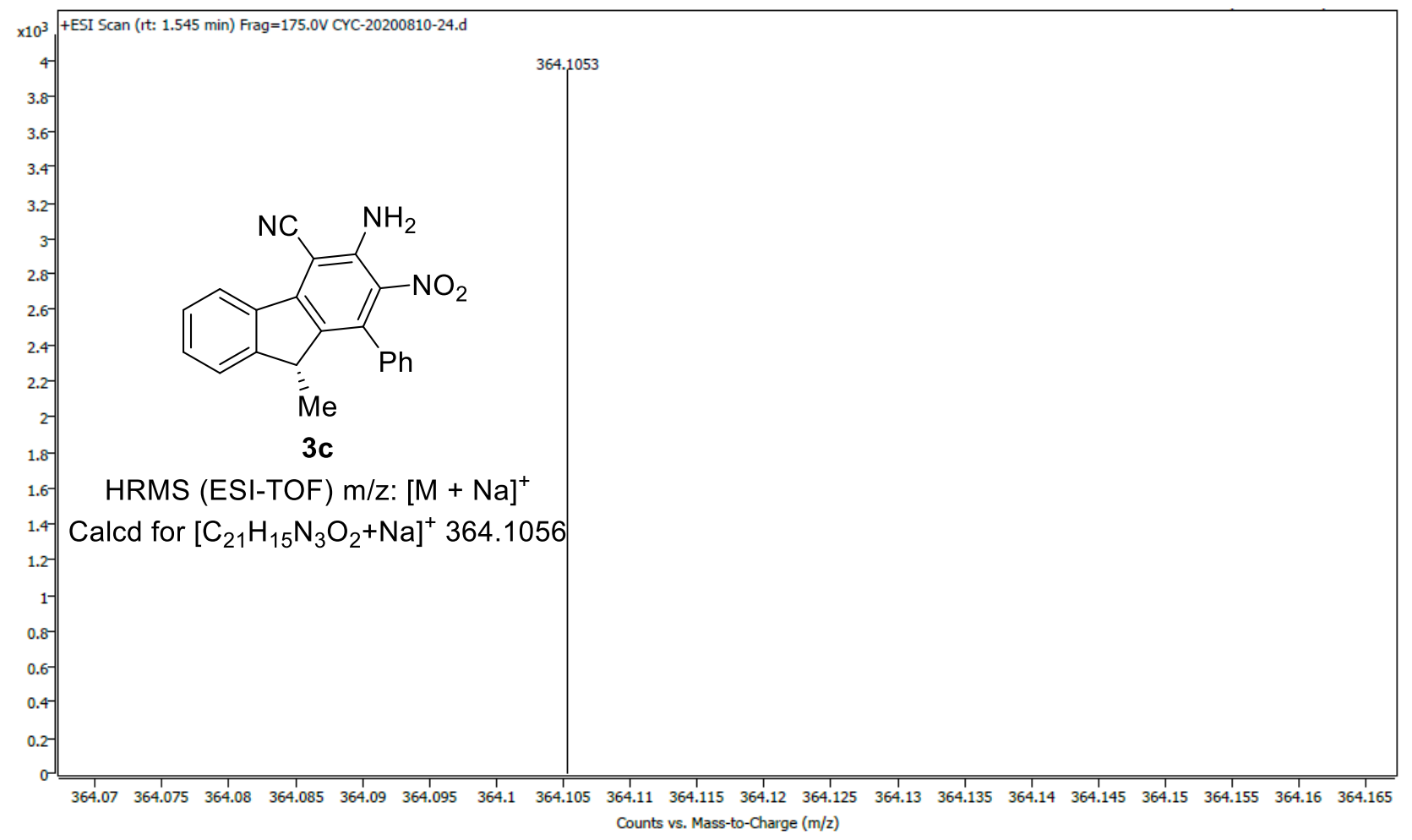




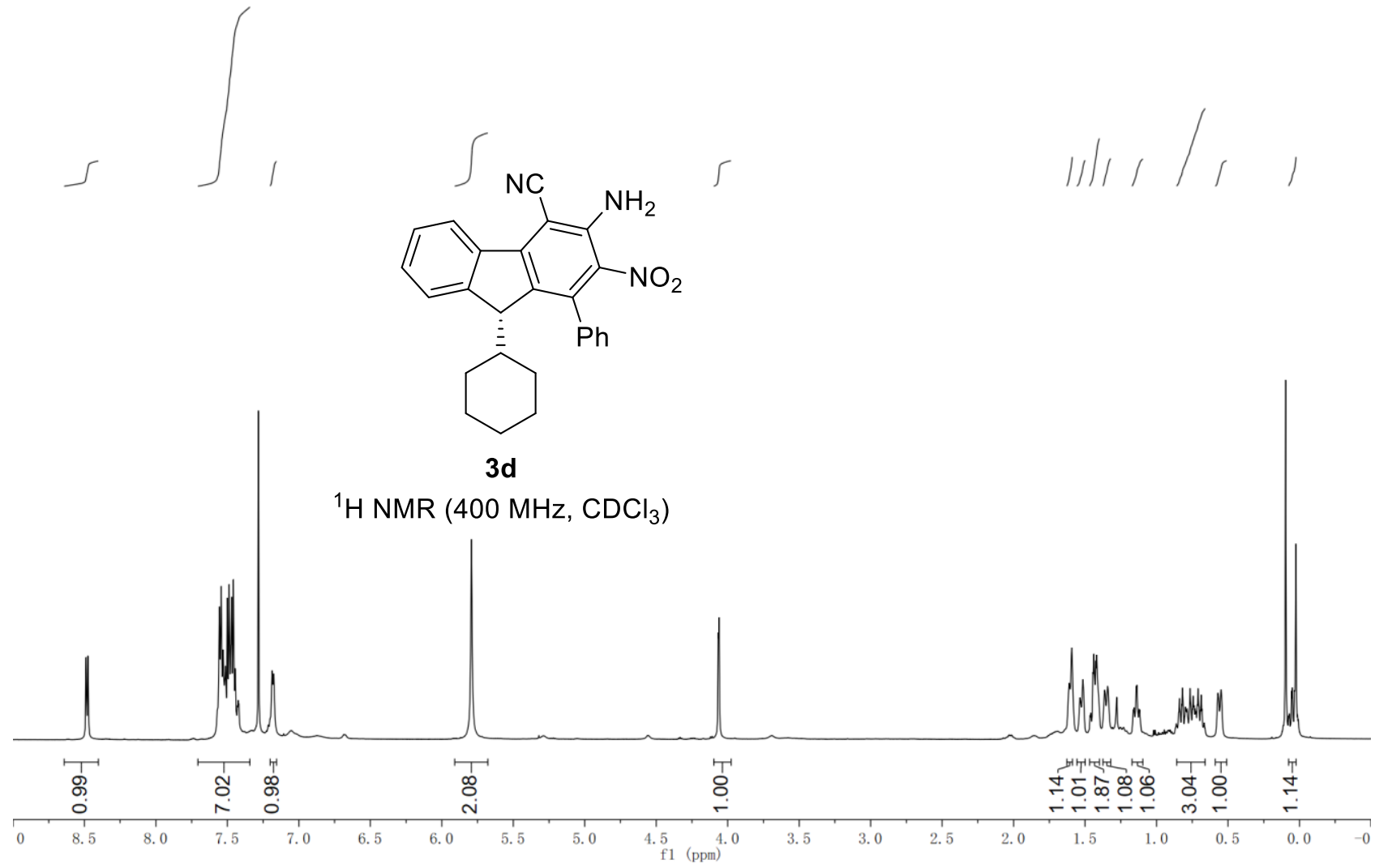<smiles>N#Cc1c(N)c([N+](=O)[O-])c(-c2ccccc2)c2c1-c1ccccc1C2C1CCCCC1</smiles>

$3 d$

${ }^{13} \mathrm{C}$ NMR $\left(100 \mathrm{MHz}, \mathrm{CDCl}_{3}\right)$
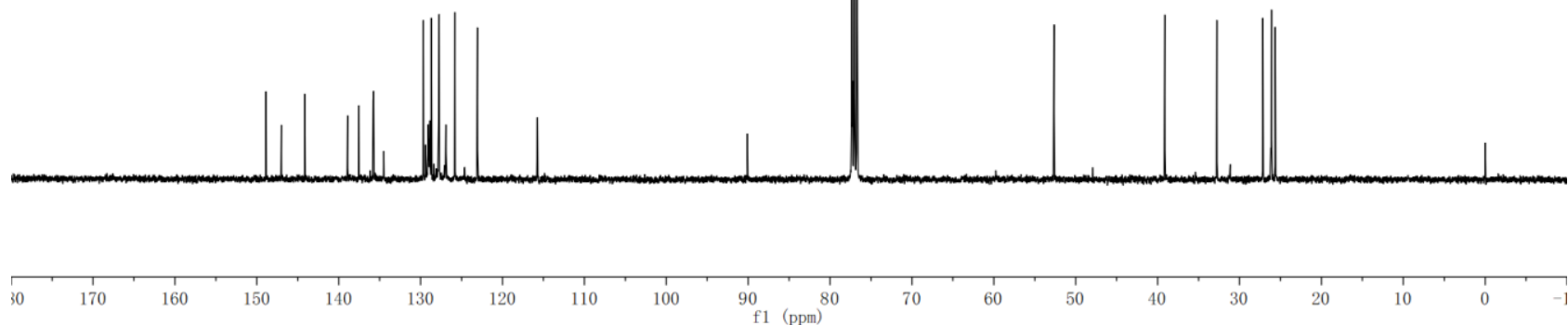


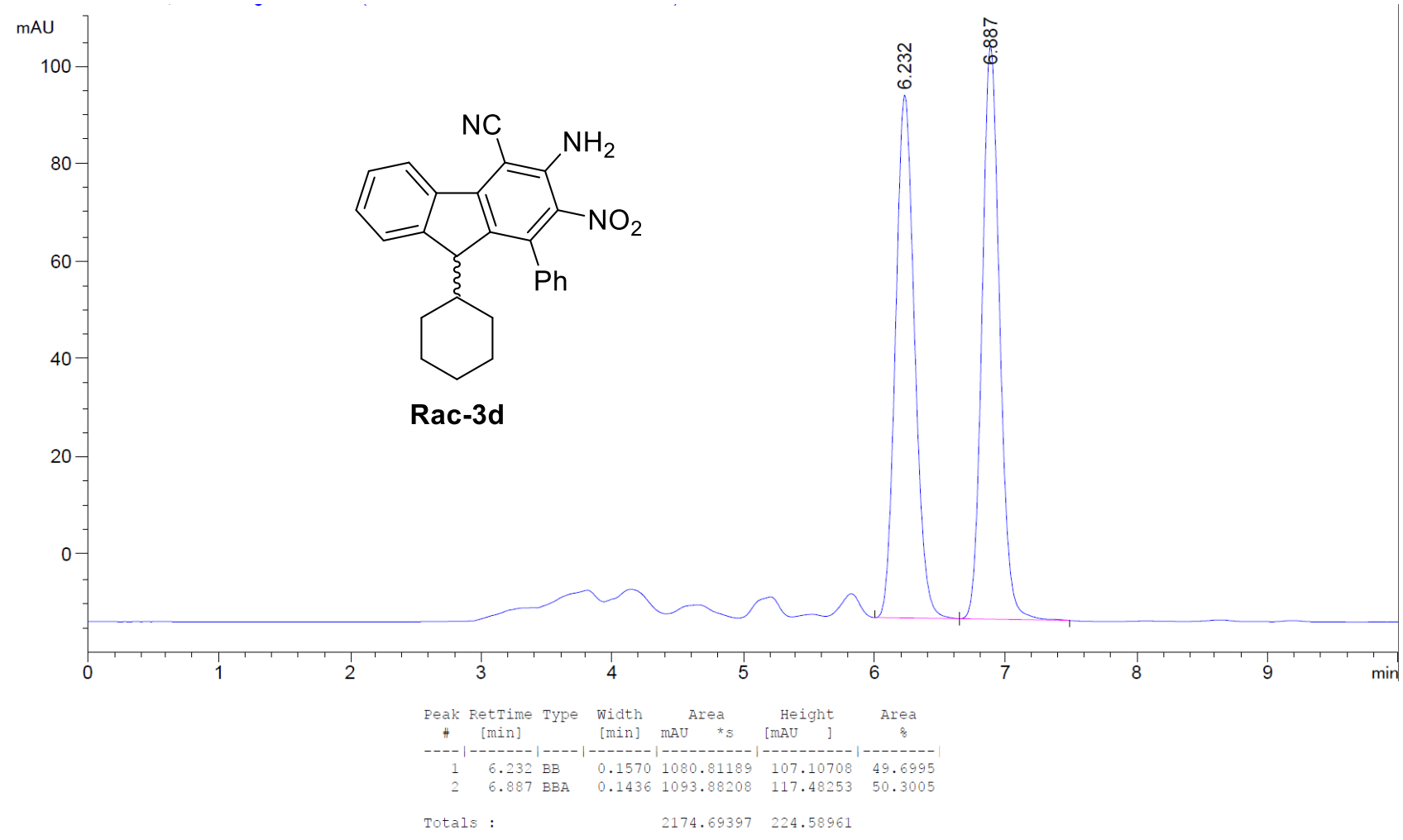

Retention Time

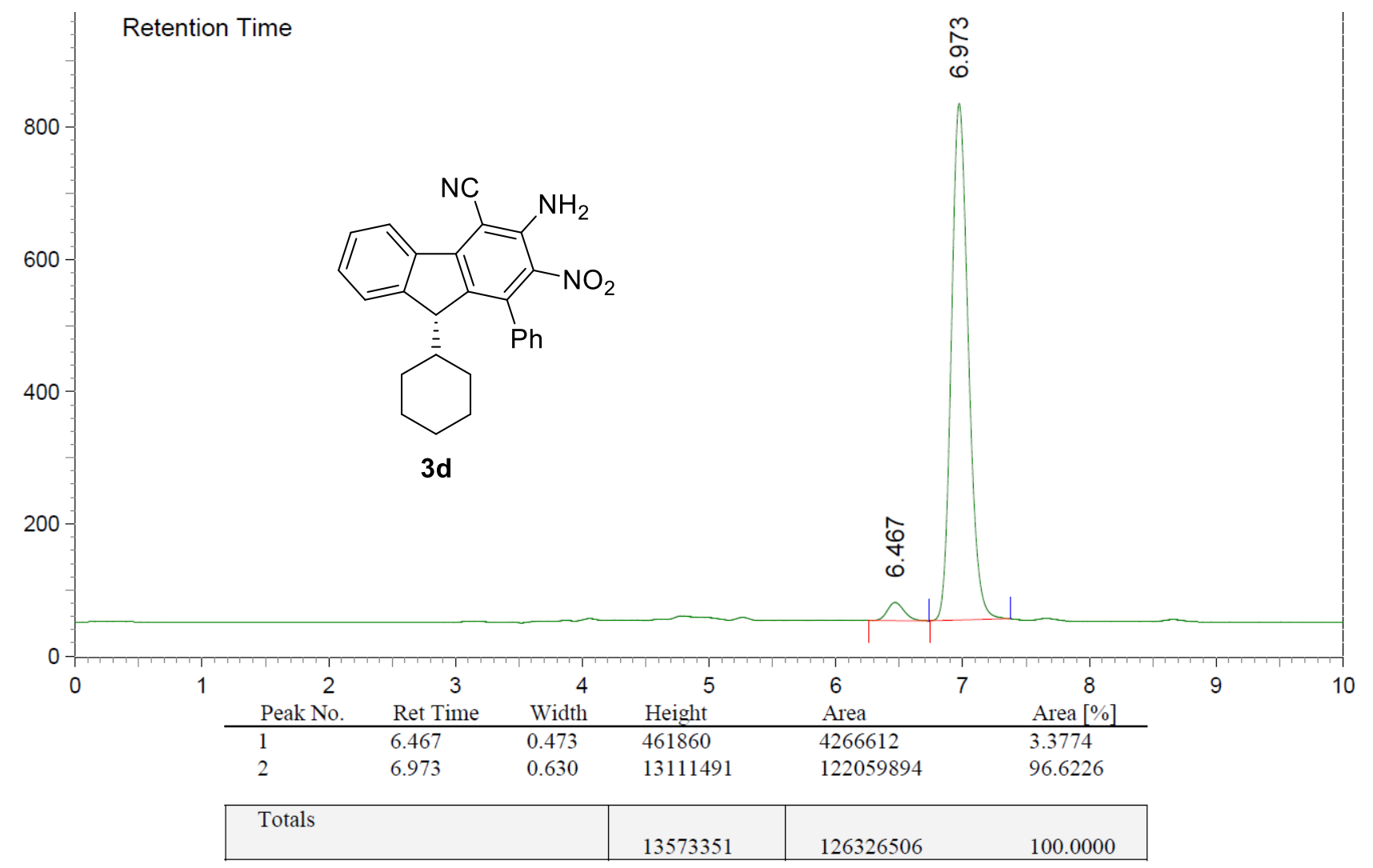




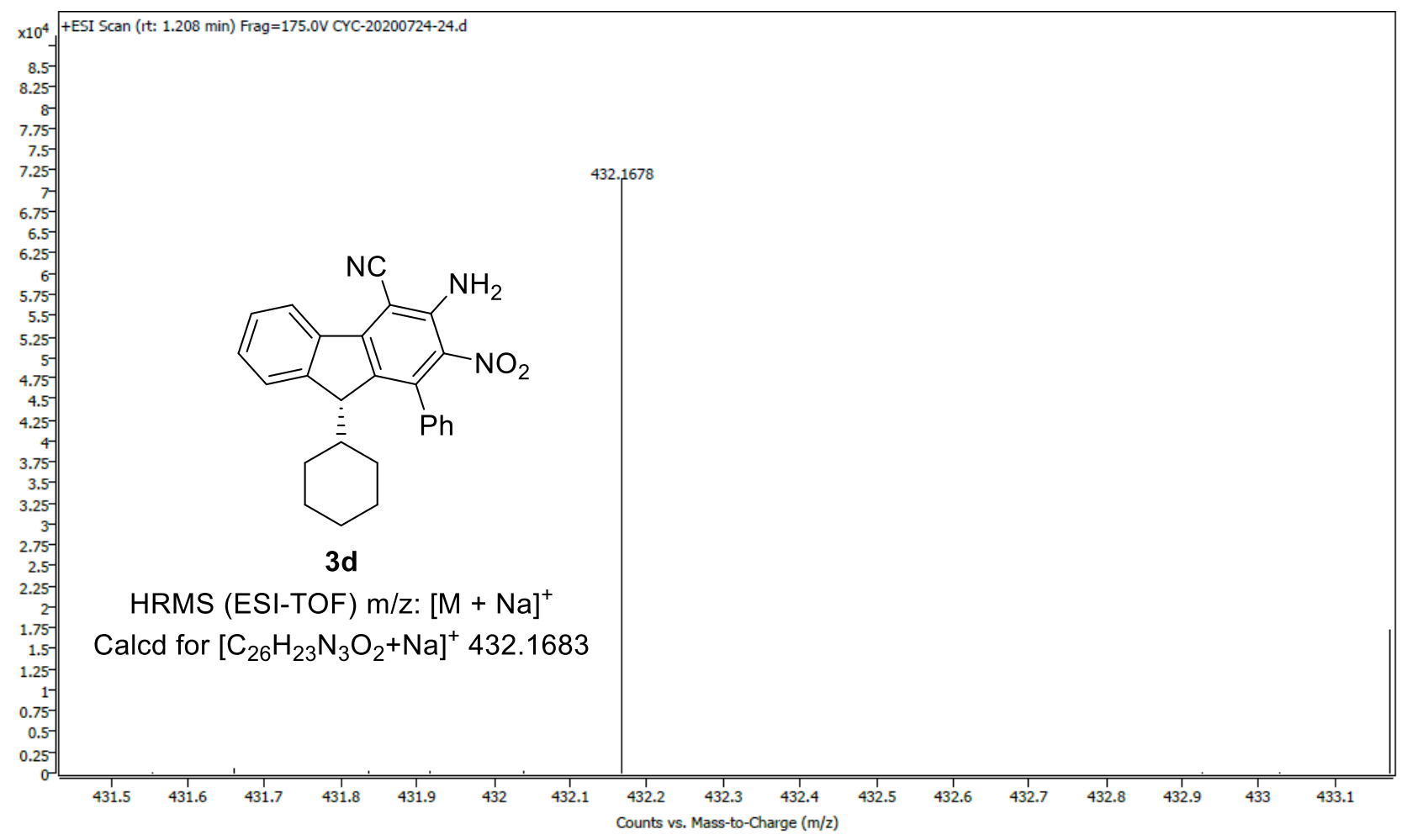



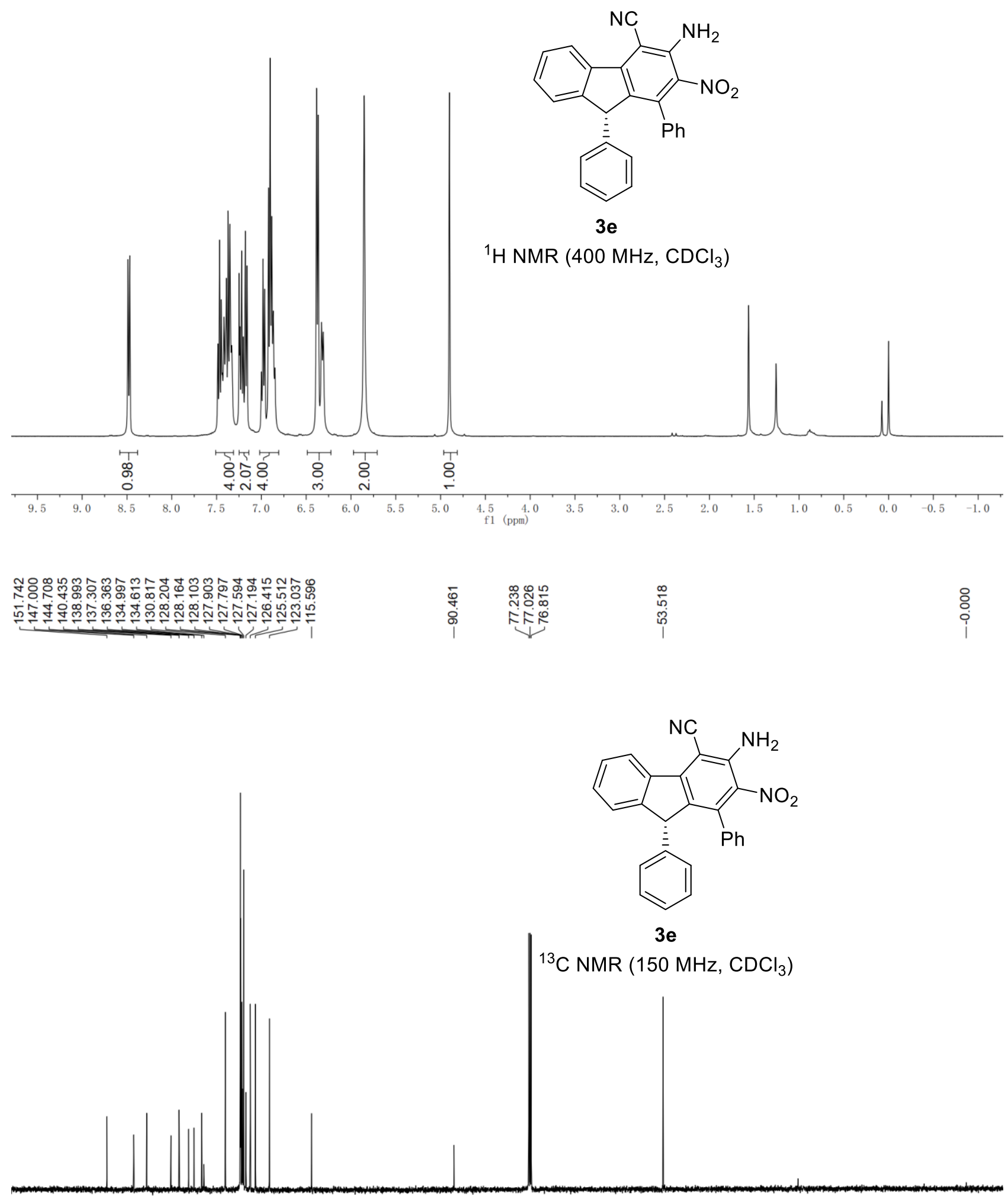


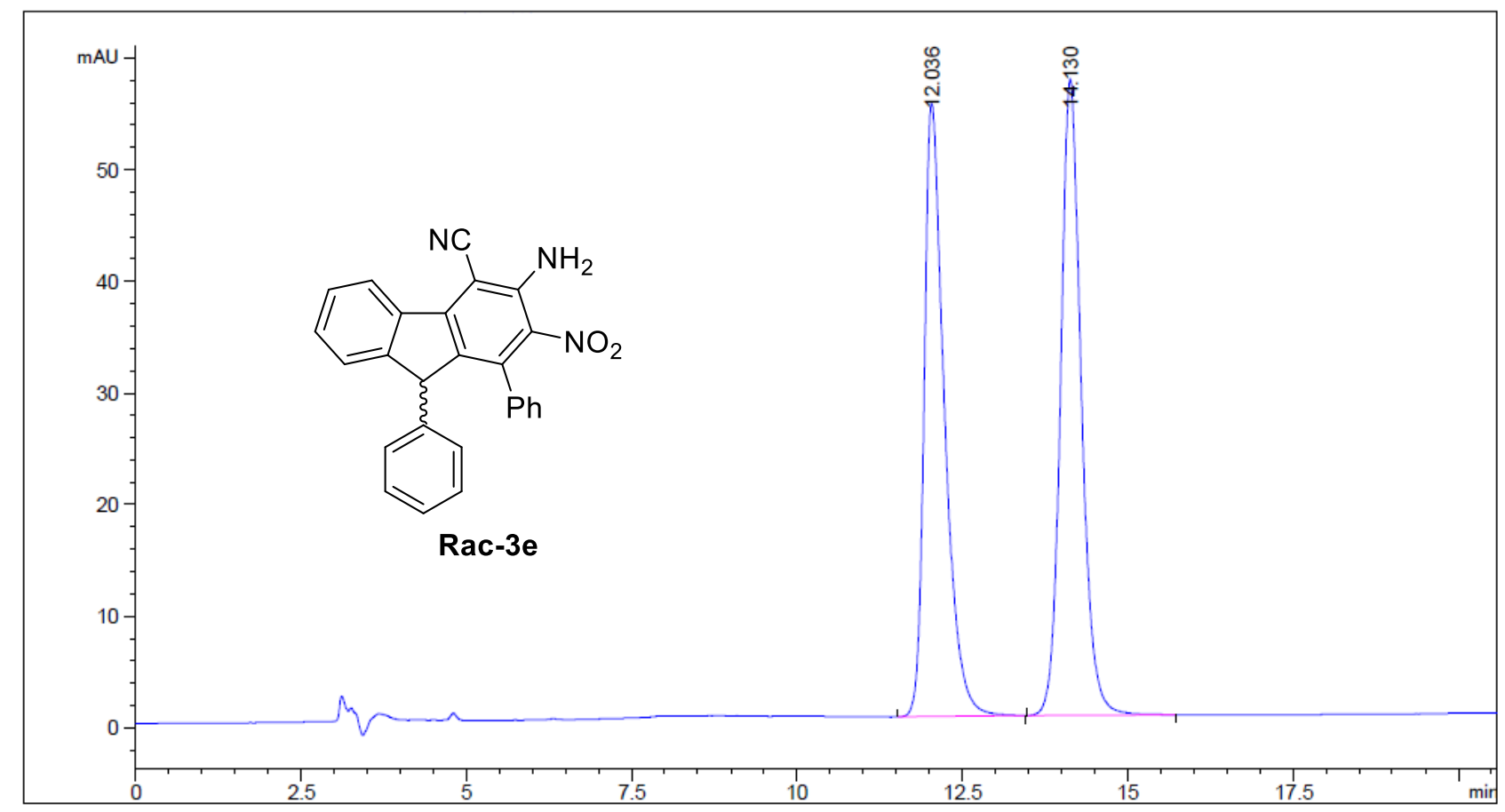

\begin{tabular}{rrrrrr} 
Peak No. & Ret Time & Width & Height & Area & Area [\%] \\
\hline 1 & 12.036 & 0.3172 & 1171.05334 & 54.92426 & 48.5982 \\
2 & 14.130 & 0.3293 & 1238.60828 & 56.97477 & 51.4018
\end{tabular}

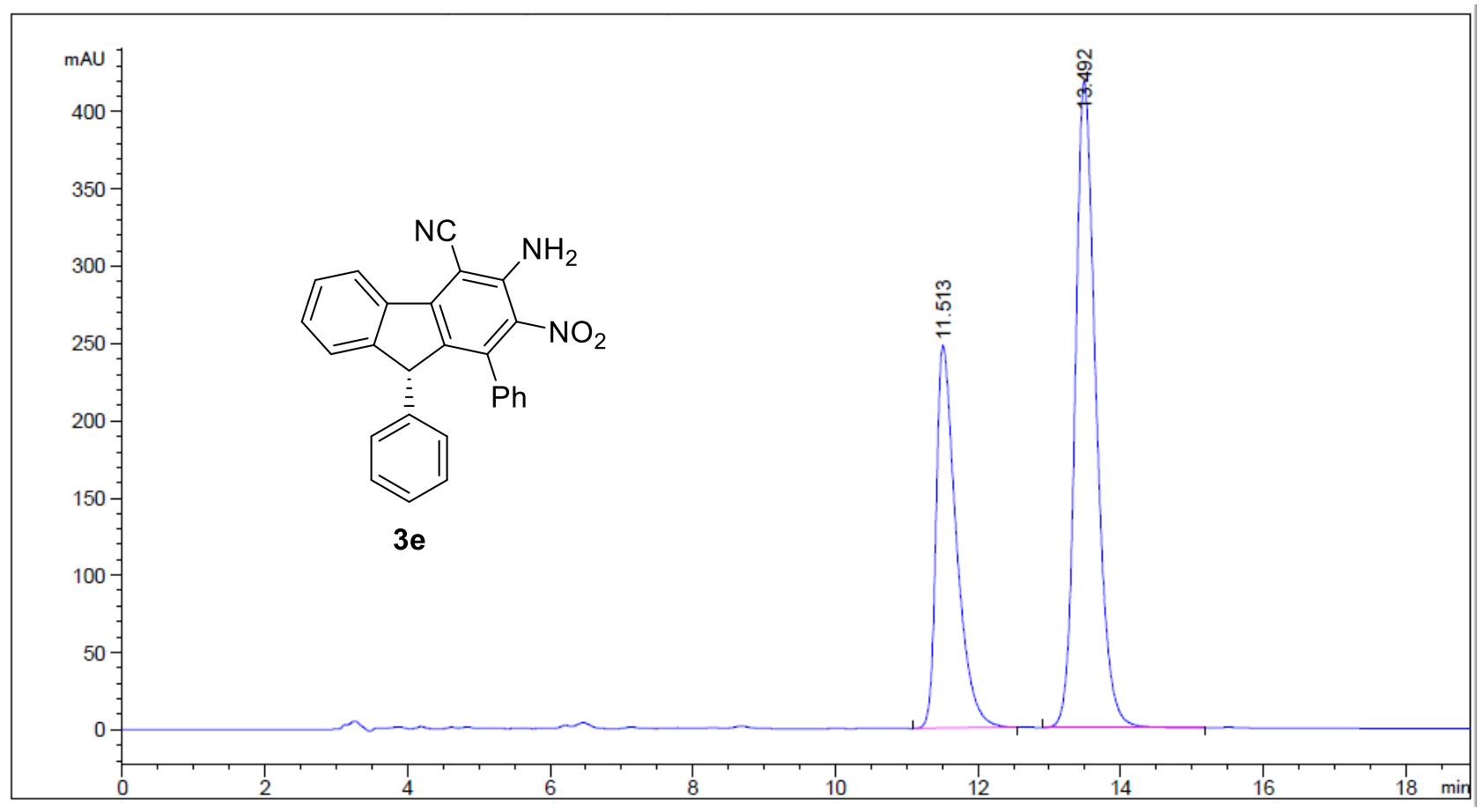

\begin{tabular}{cccccc} 
Peak No. & Ret Time & Width & Height & Area & Area [\%] \\
\hline 1 & 11.513 & 0.2936 & 4870.72607 & 247.55792 & 36.4726 \\
2 & 13.492 & 0.3078 & 8483.76660 & 418.56955 & 63.5274
\end{tabular}




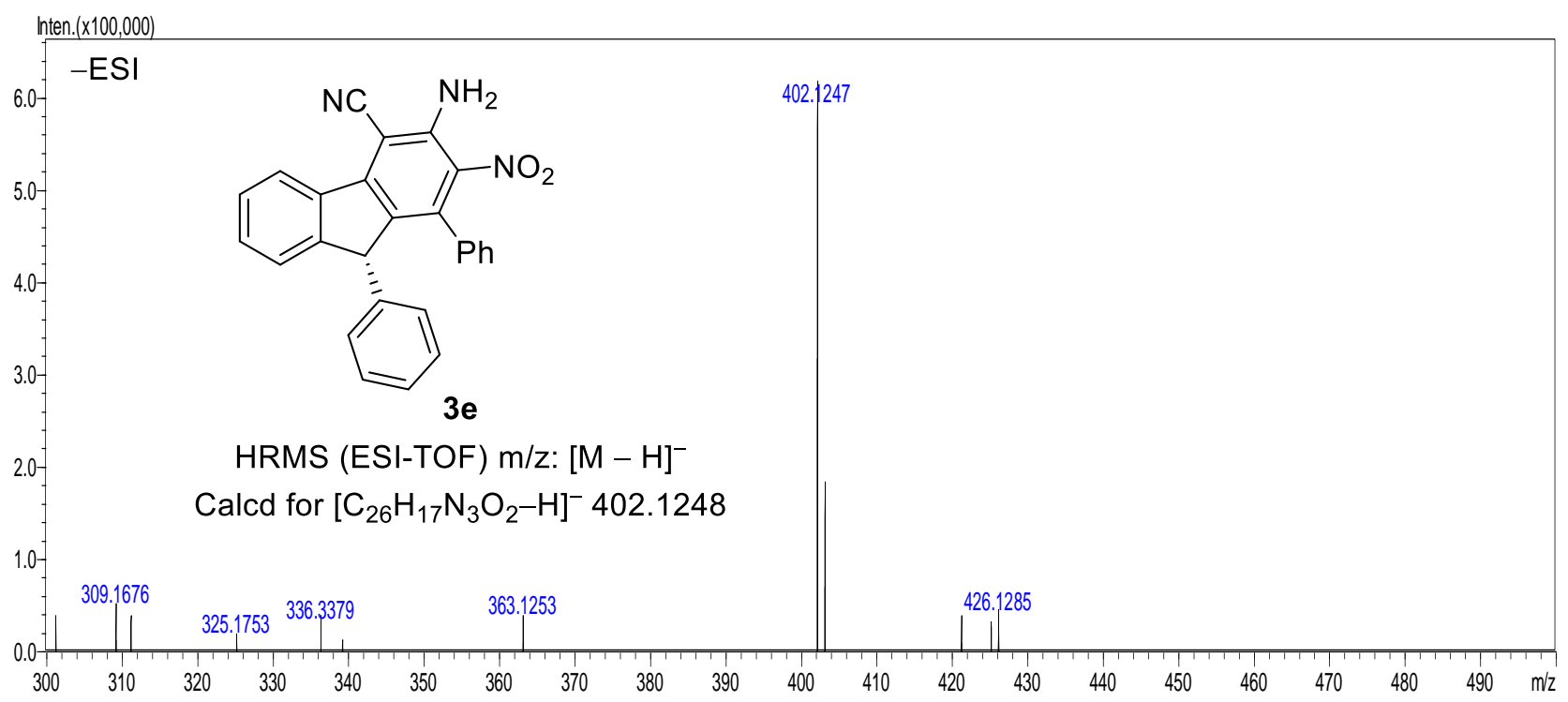




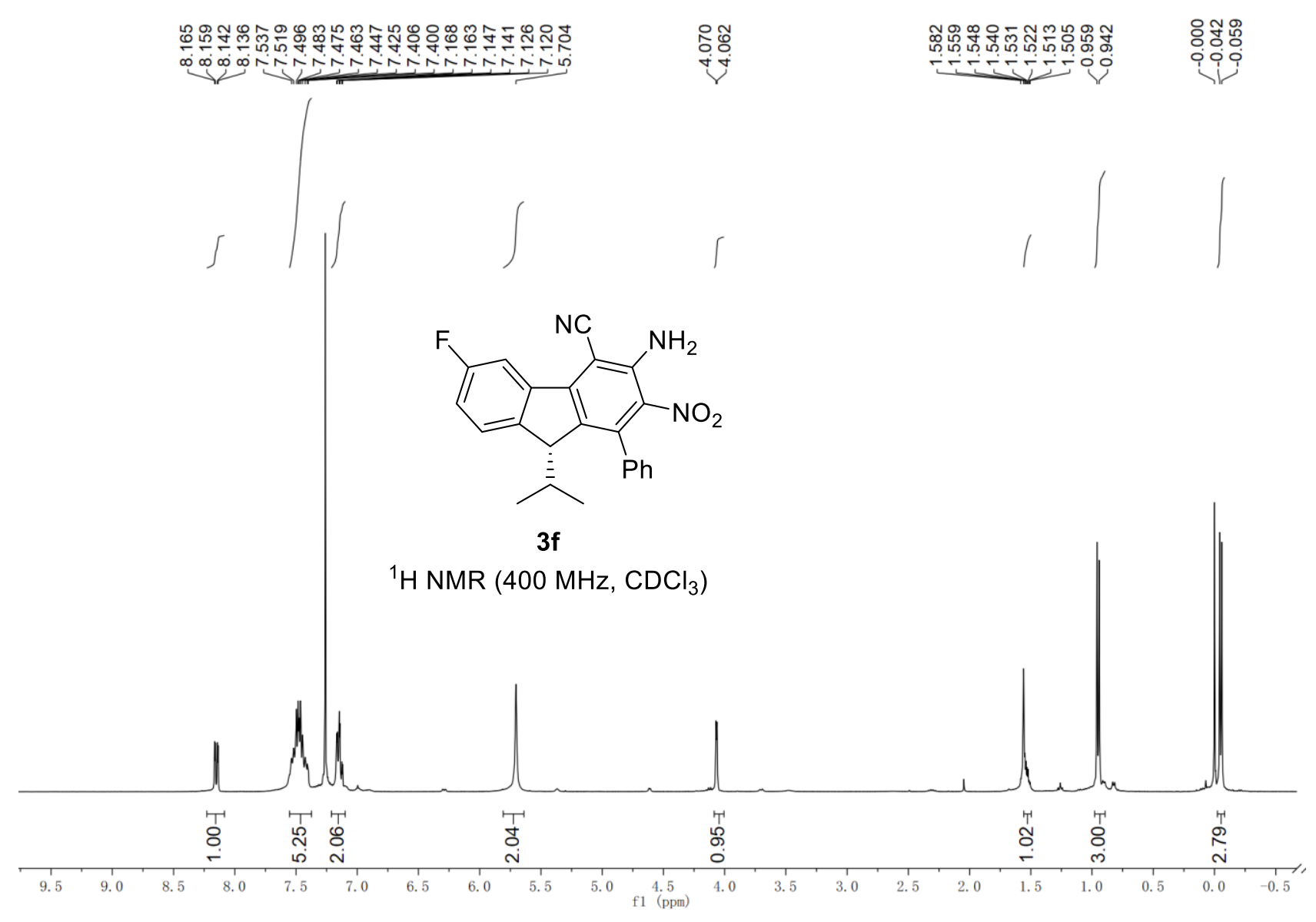

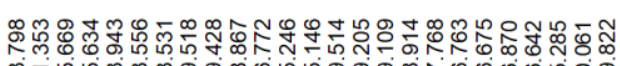

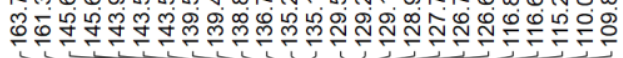

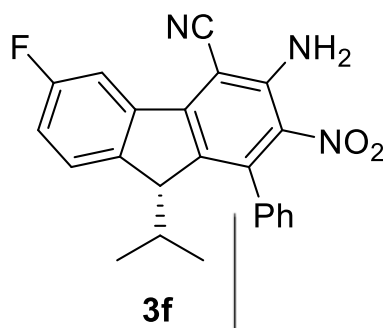

${ }^{13} \mathrm{C}$ NMR $\left(100 \mathrm{MHz}, \mathrm{CDCl}_{3}\right)$

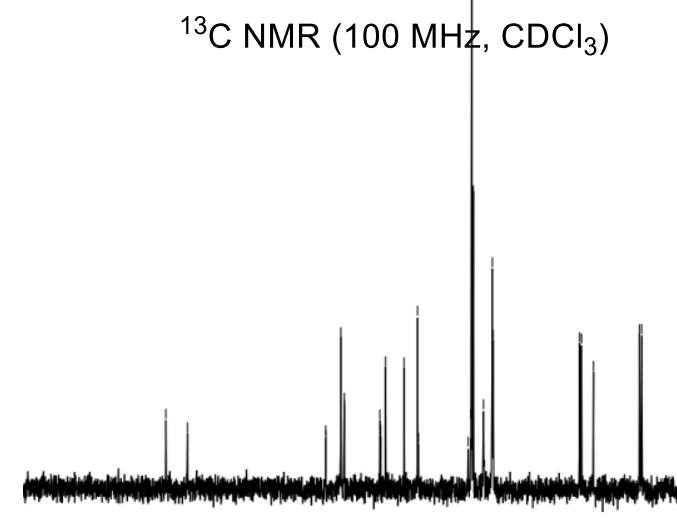

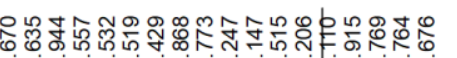

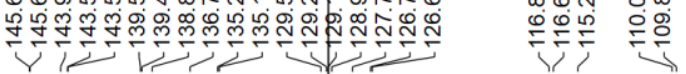
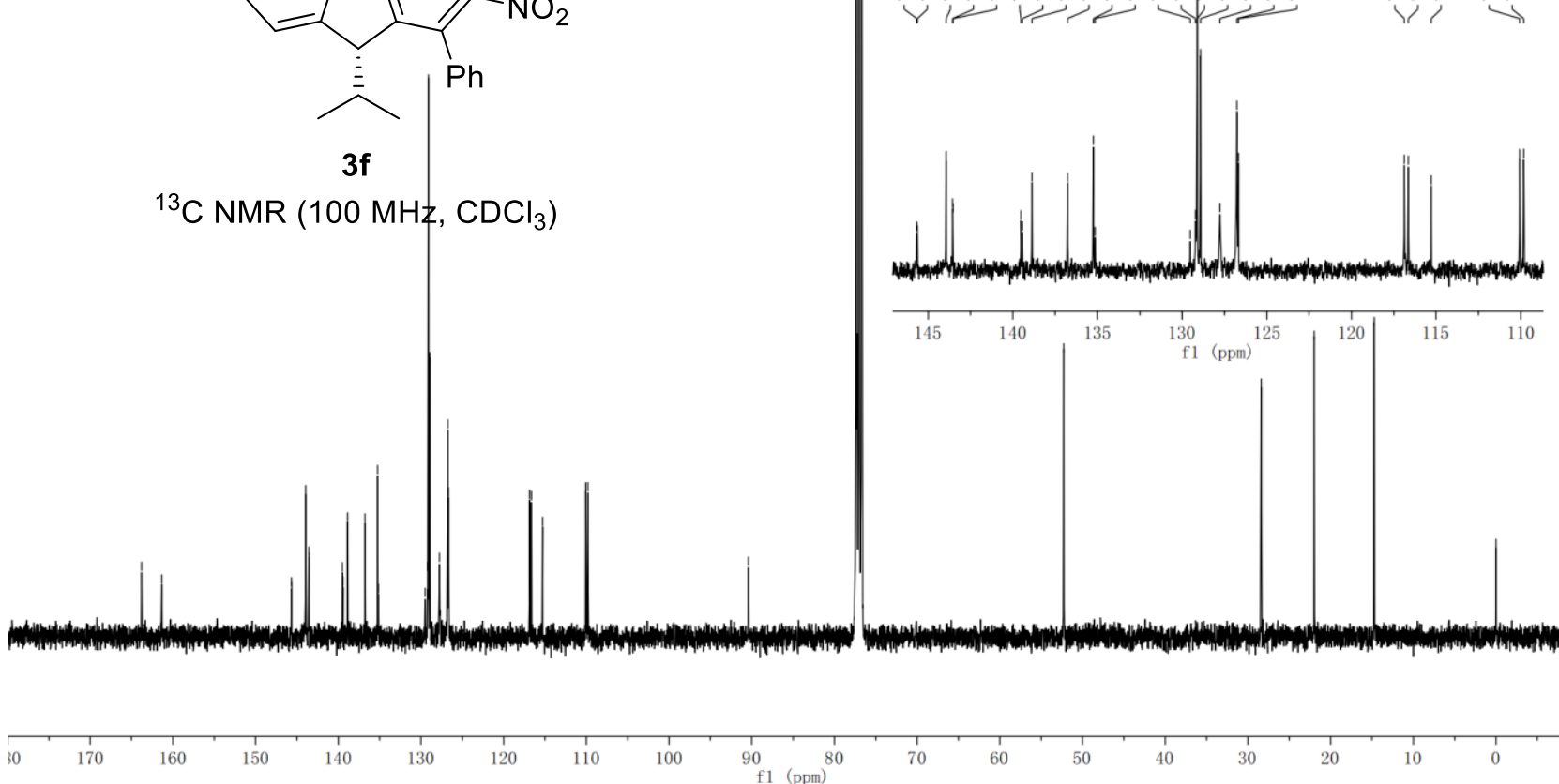


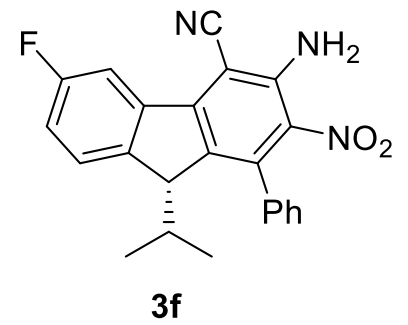

${ }^{19} \mathrm{~F} \mathrm{NMR}\left(376 \mathrm{MHz}, \mathrm{CDCl}_{3}\right)$

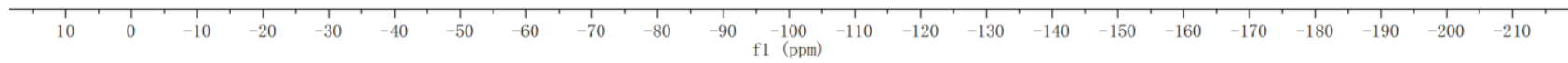

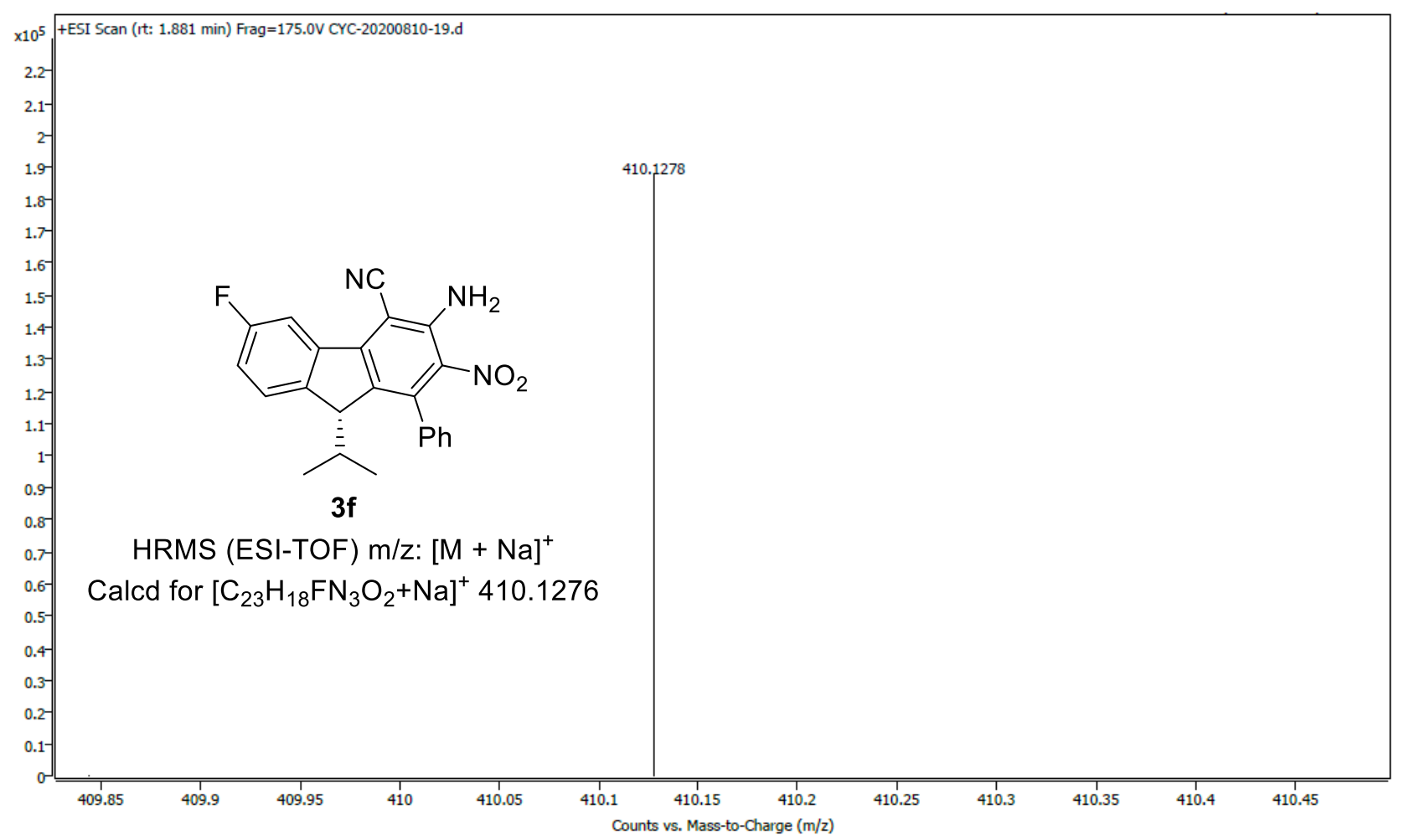



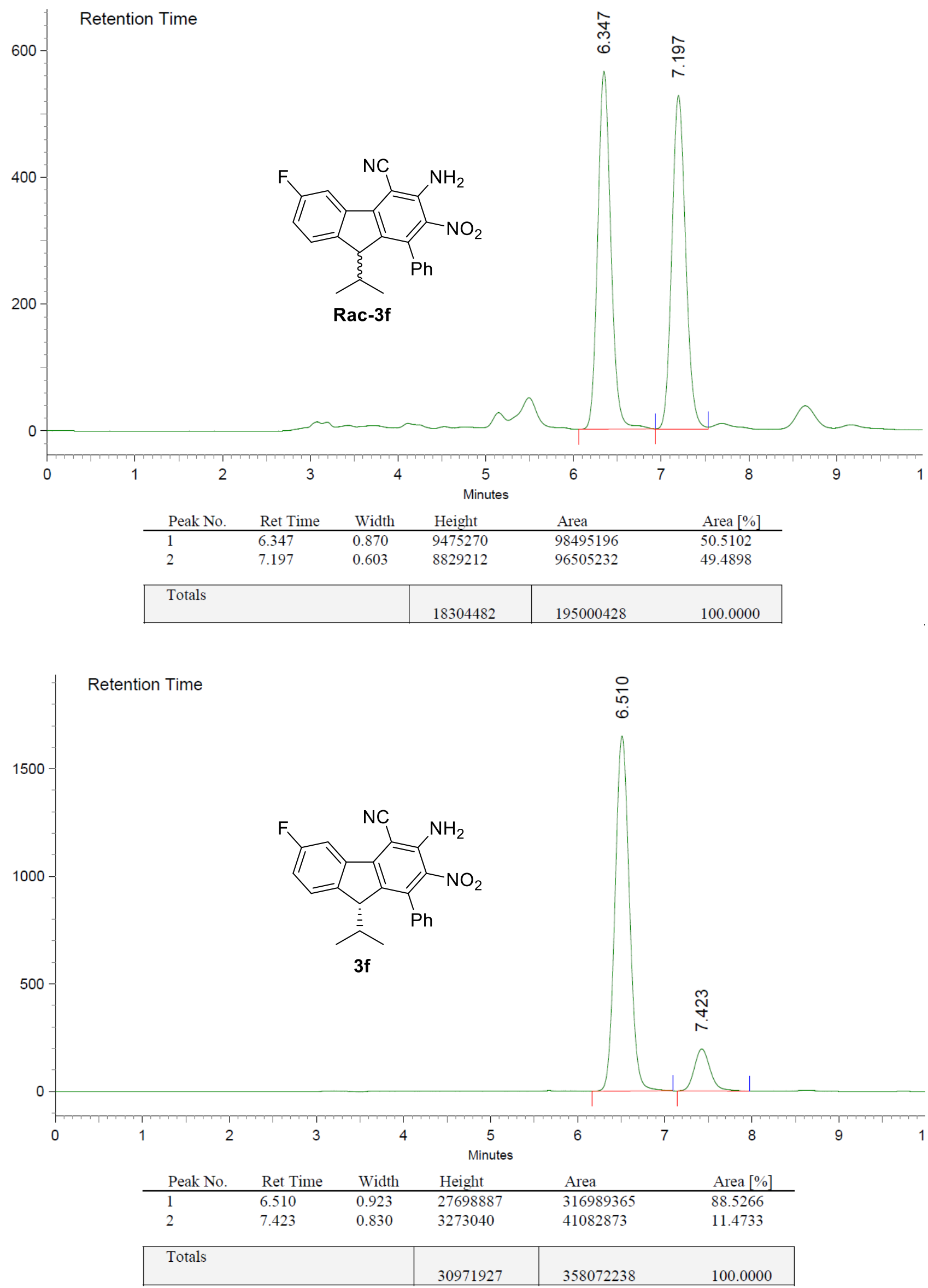

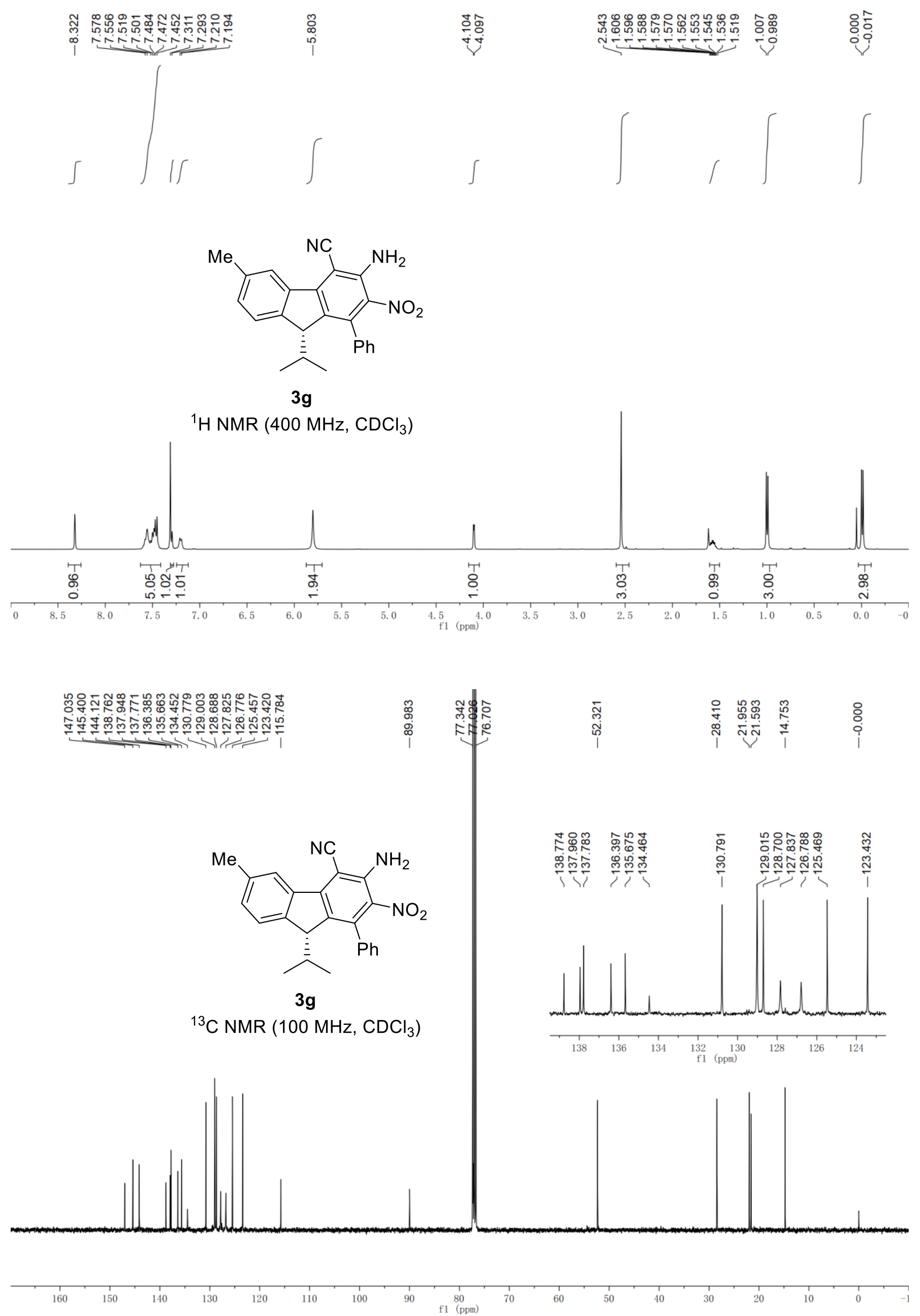

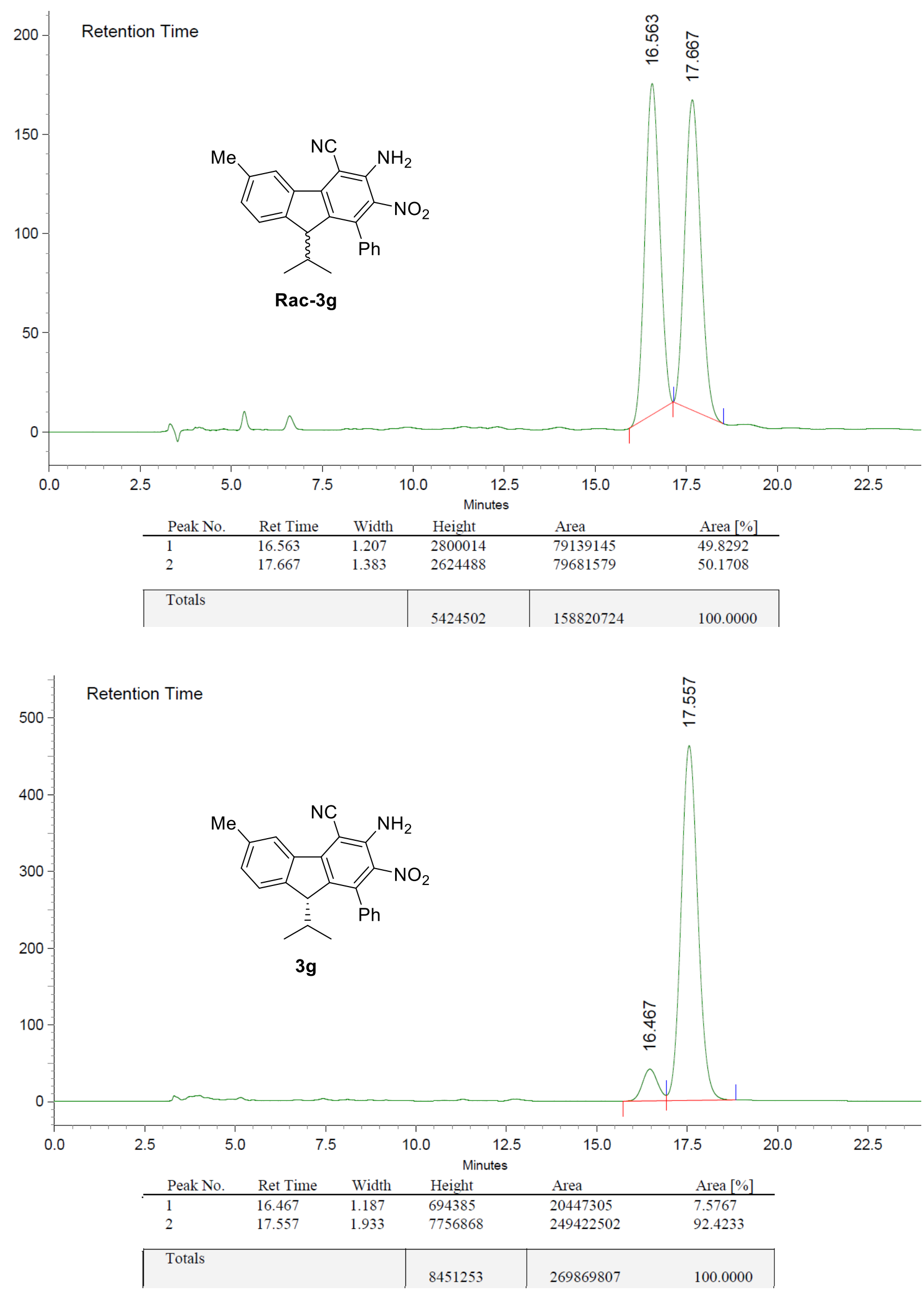


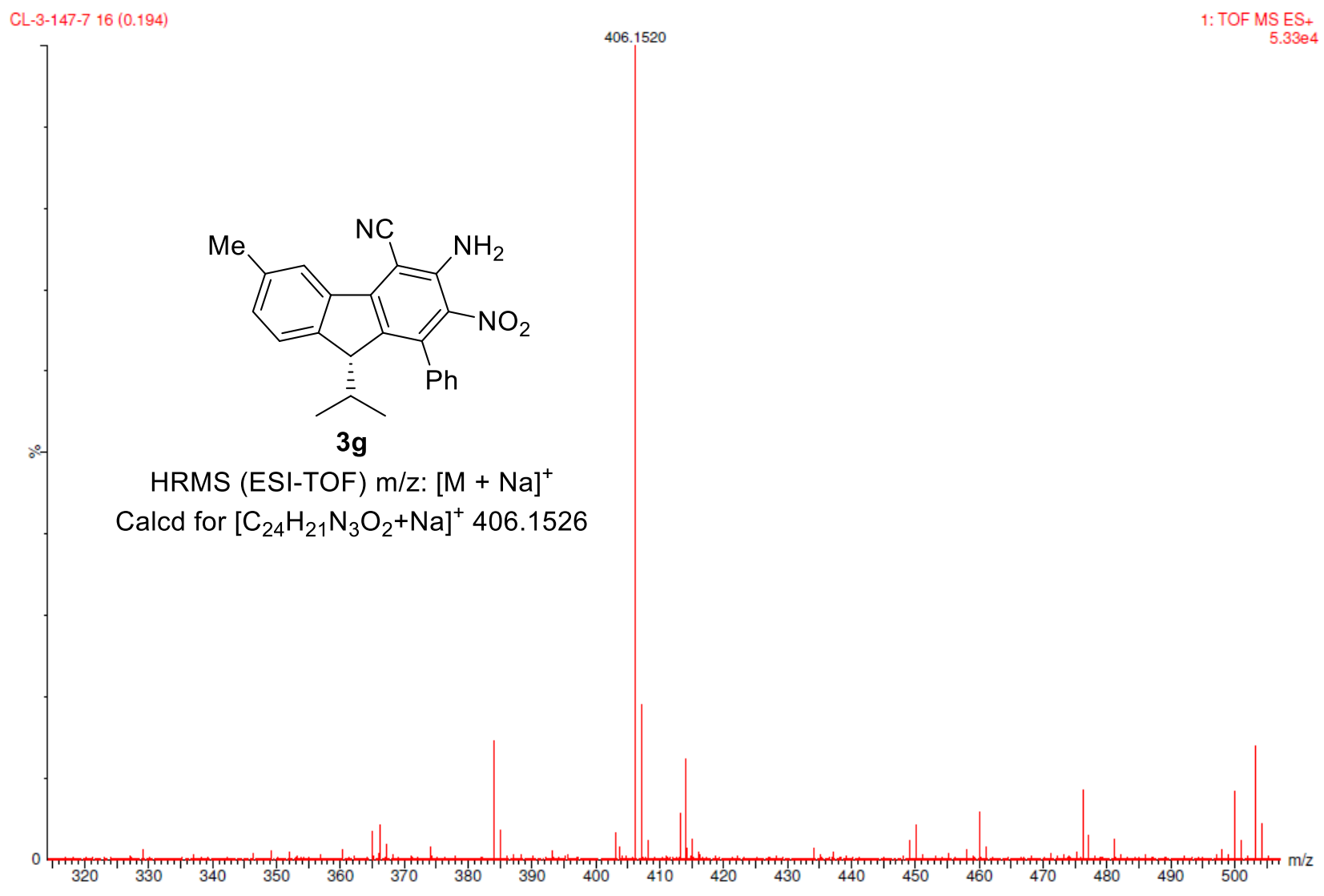




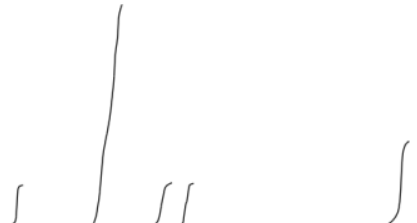<smiles>COc1ccc2c(c1)-c1c(C#N)c(N)c([N+](=O)[O-])c(-c3ccccc3)c1[C@H]2C(C)C</smiles>

$3 \mathbf{h}$

${ }^{1} \mathrm{H}$ NMR $\left(400 \mathrm{MHz}, \mathrm{CDCl}_{3}\right)$

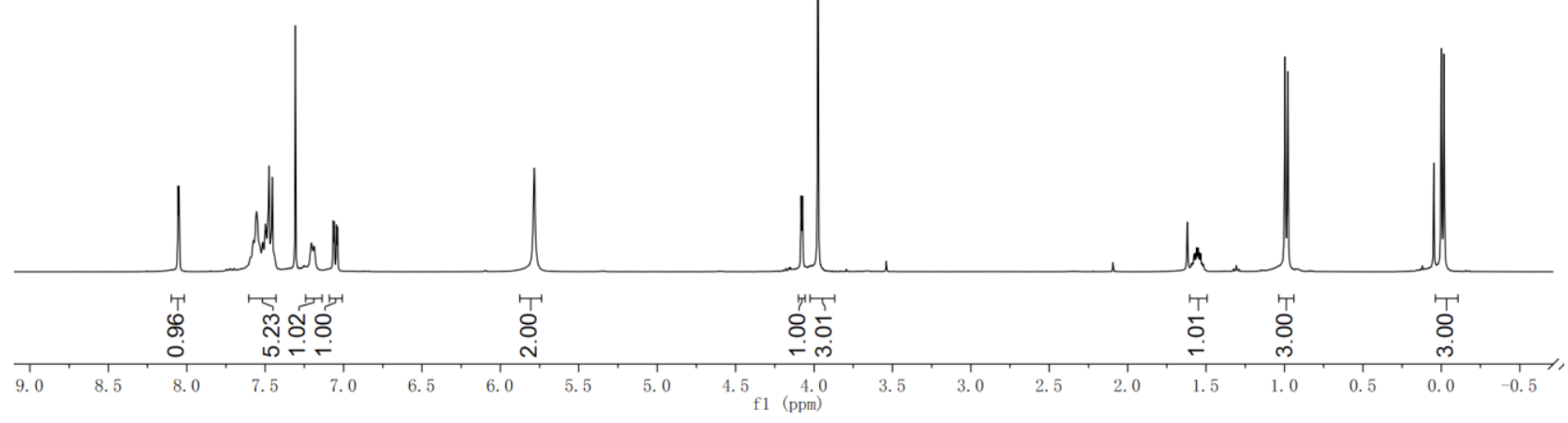

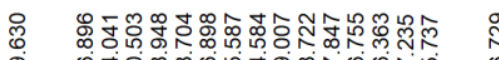

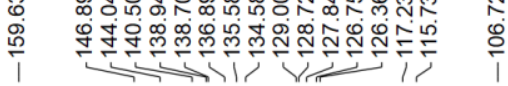<smiles>COc1ccc2c(c1)-c1c(C#N)c(N)c([N+](=O)[O-])c(-c3ccccc3)c1[C@H]2C(C)C</smiles>

$3 \mathrm{~h}$

${ }^{13} \mathrm{C}$ NMR $\left(100 \mathrm{MHz}, \mathrm{CDCl}_{3}\right)$

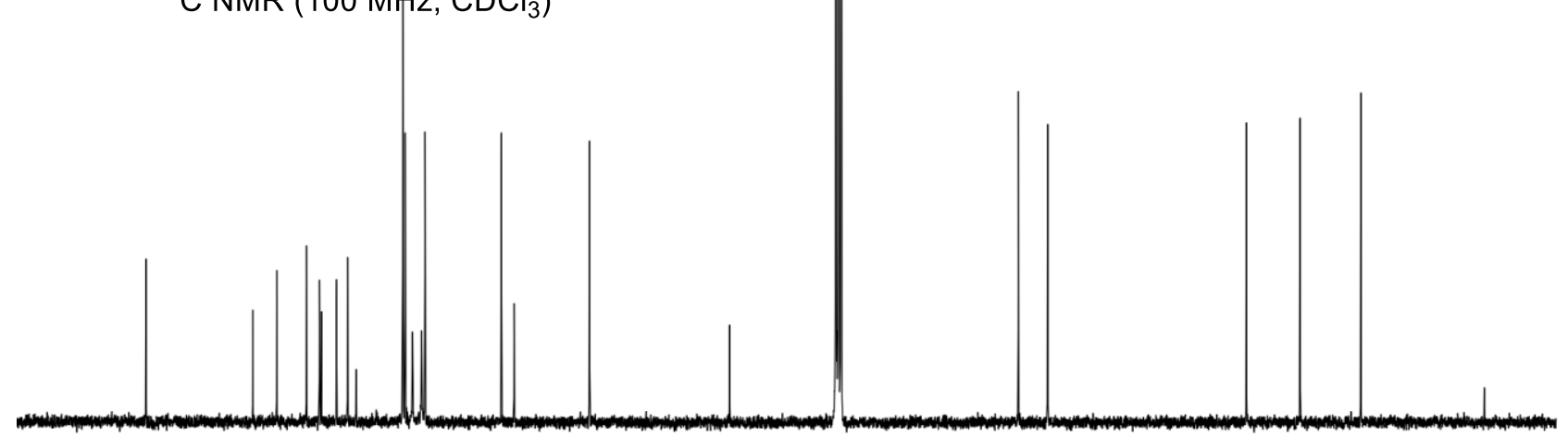

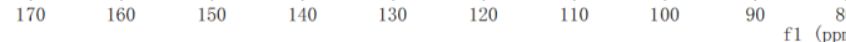



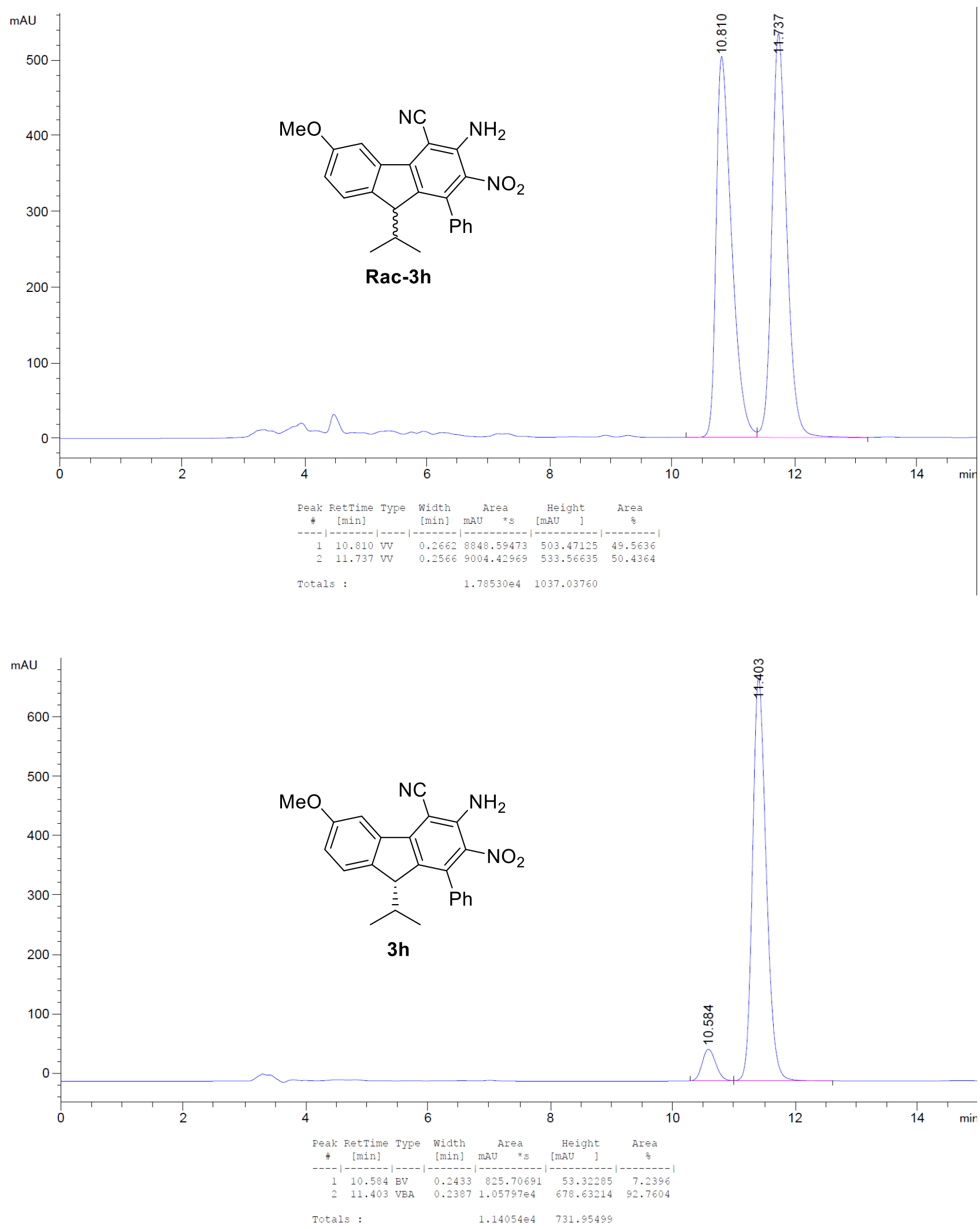


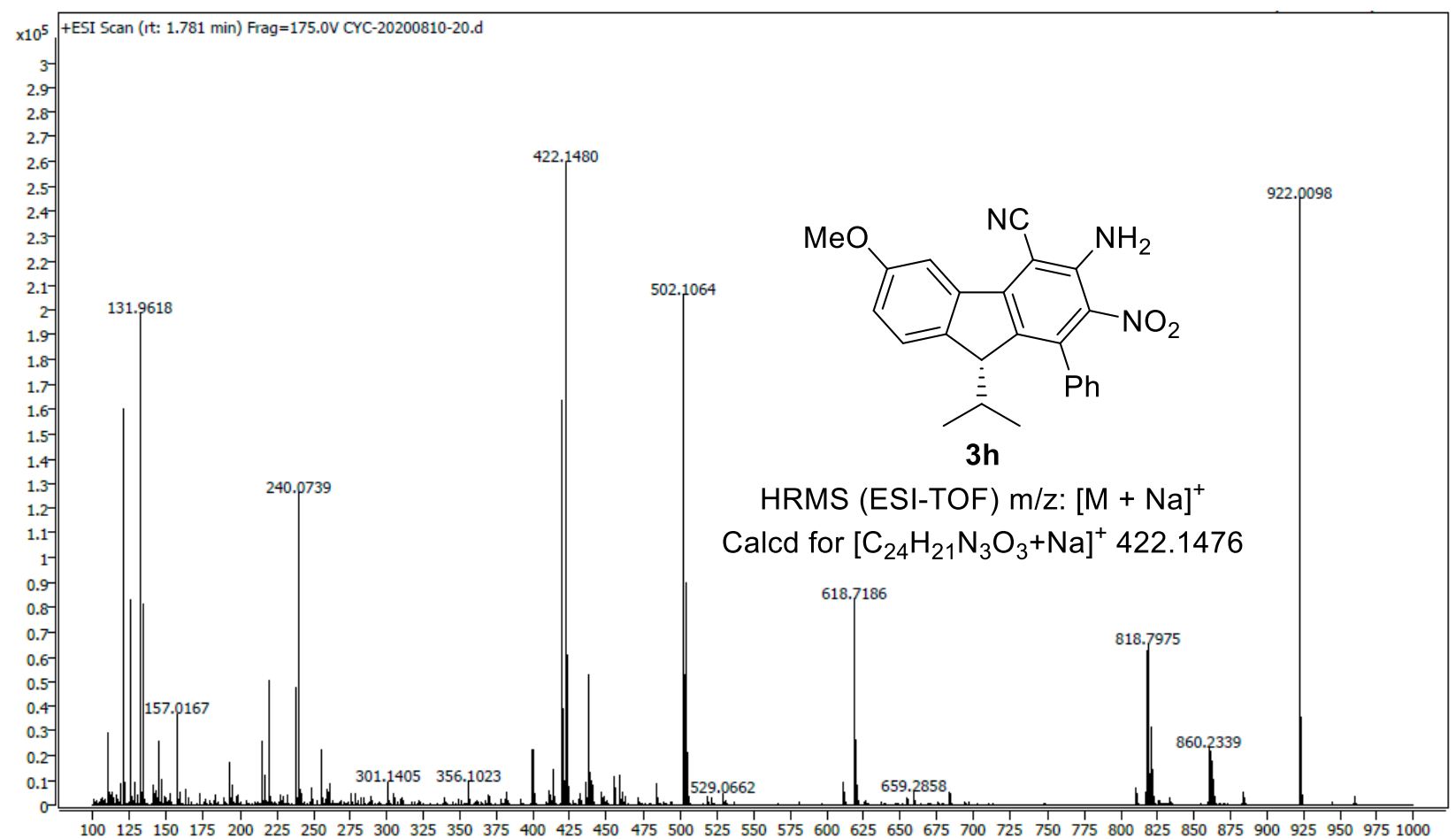



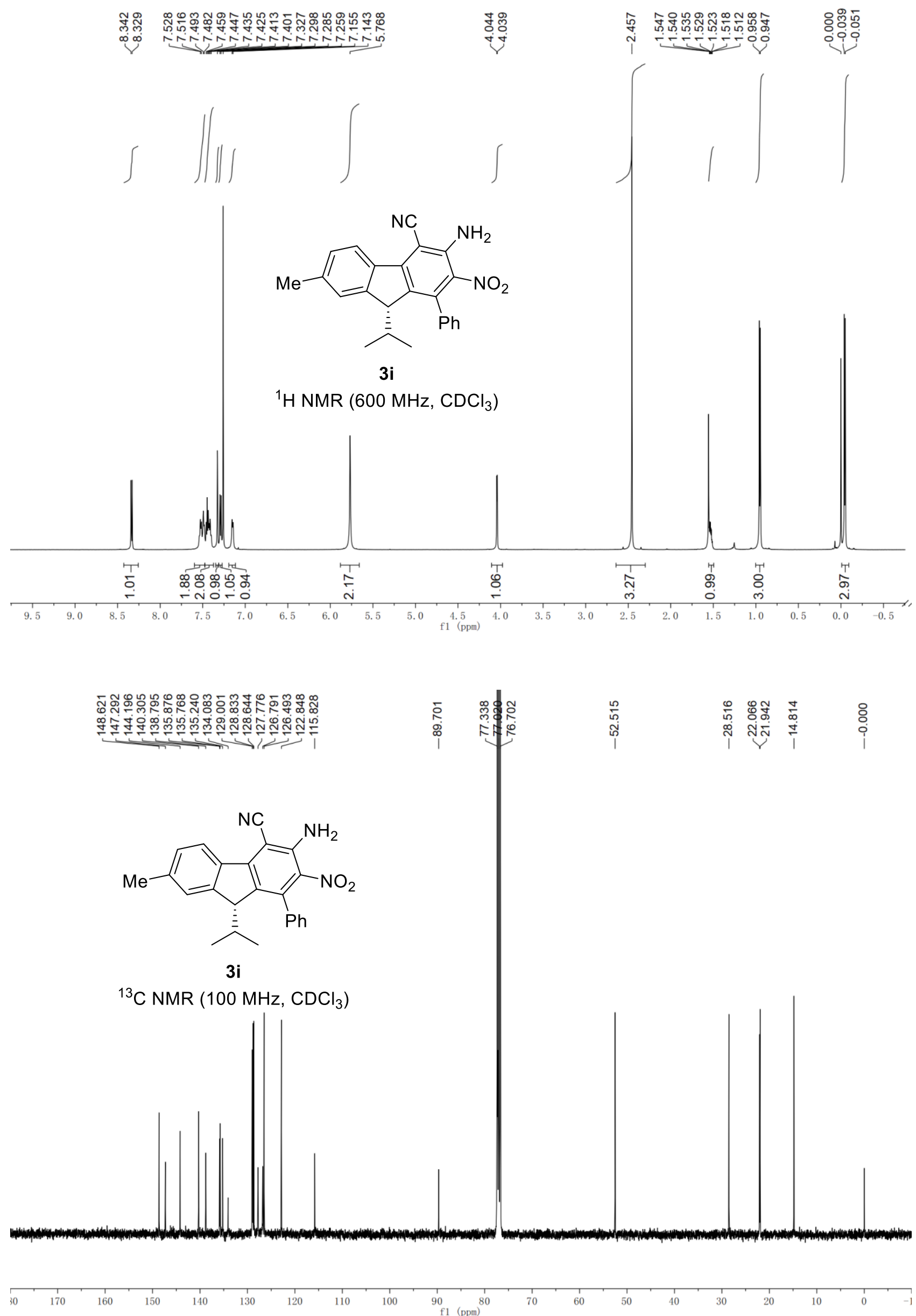

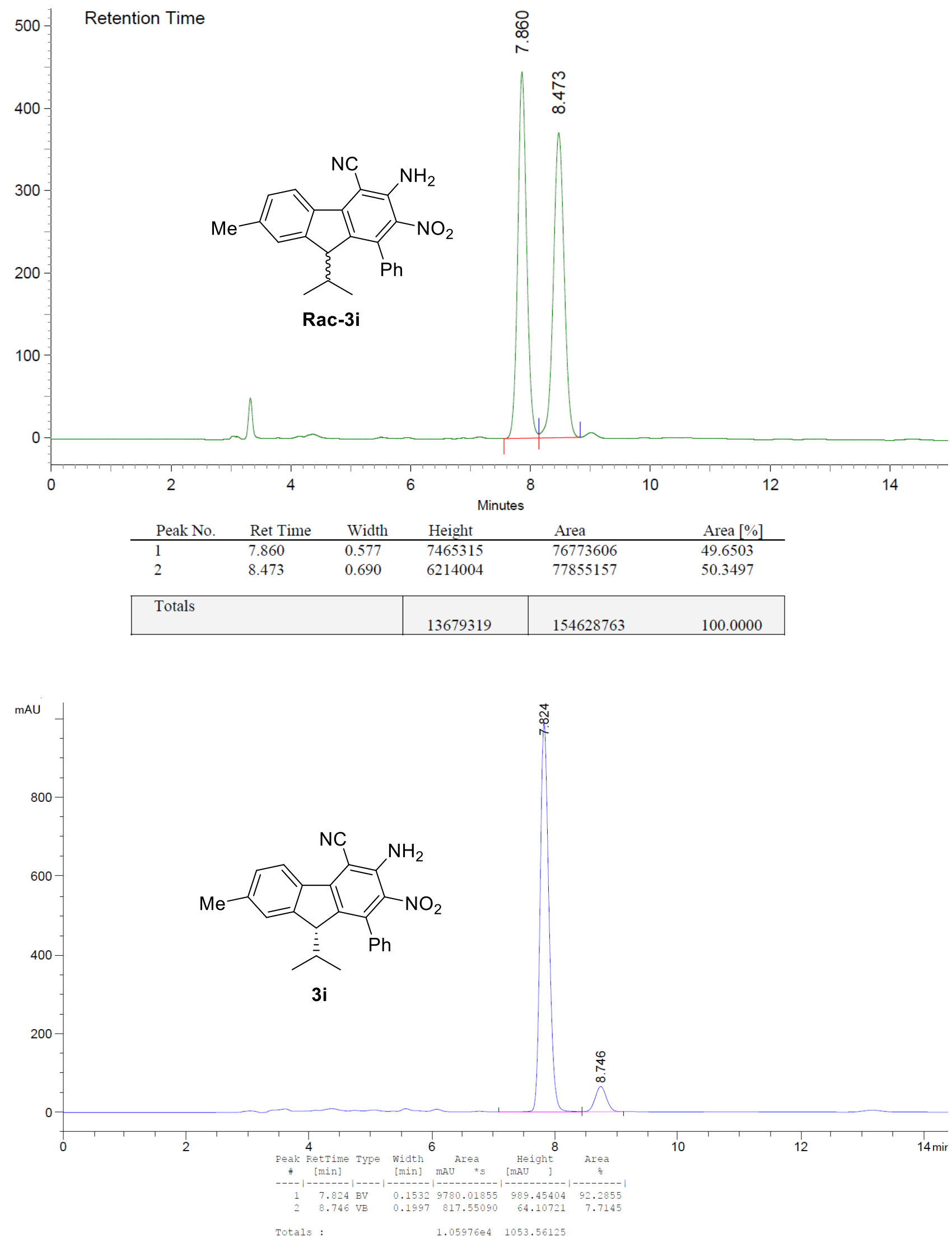


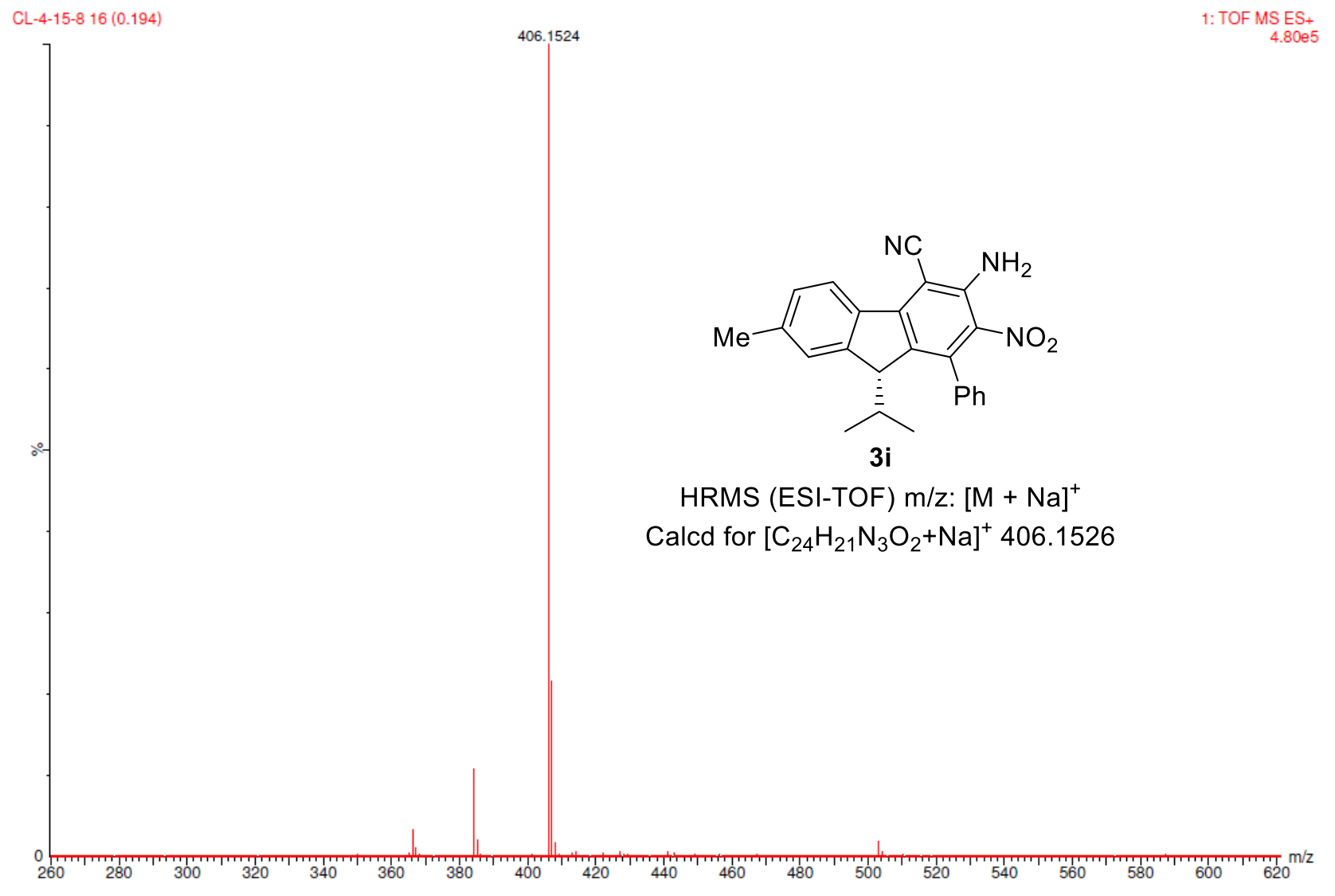




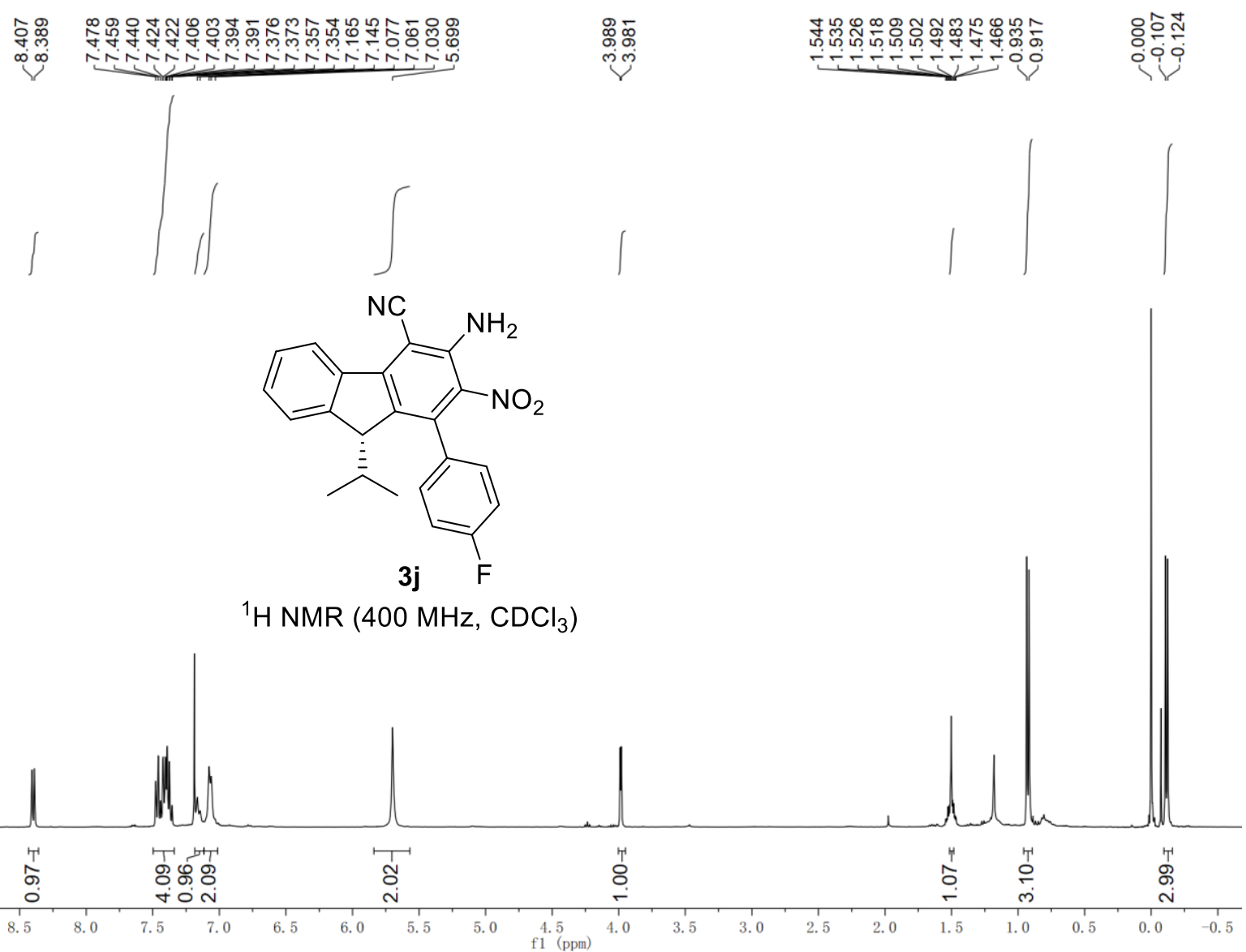

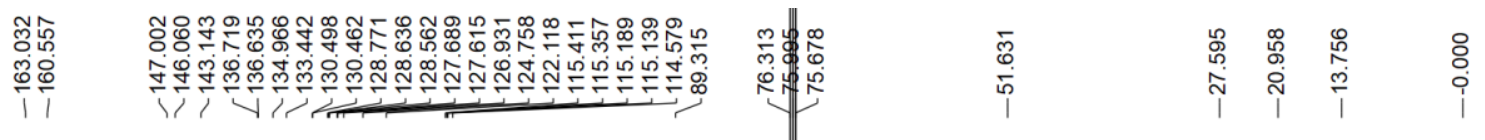<smiles>CC(C)[C@H]1c2ccccc2-c2c(C#N)c(N)c([N+](=O)[O-])c(-c3ccc(F)cc3)c21</smiles>

3j

${ }^{13} \mathrm{C} \mathrm{NMR}\left(100 \mathrm{MHz}, \mathrm{CDCl}_{3}\right)$

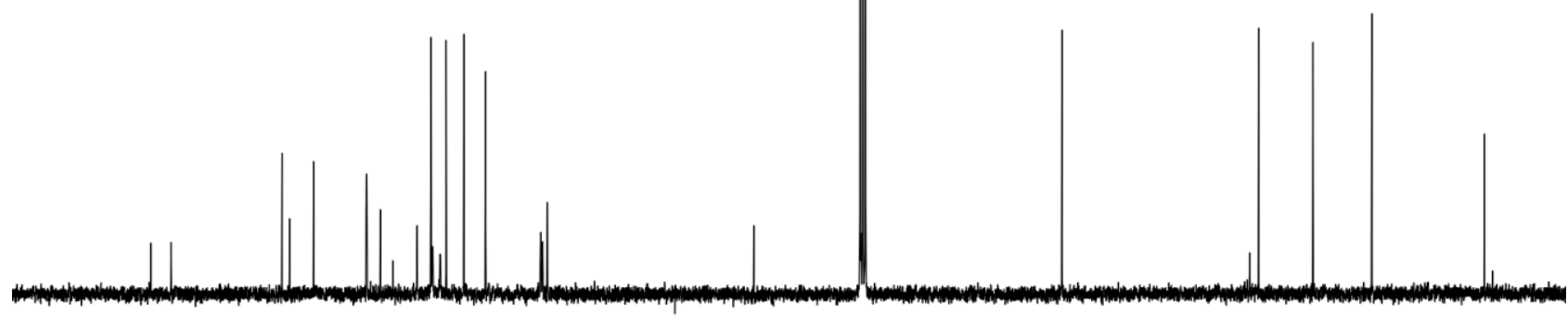

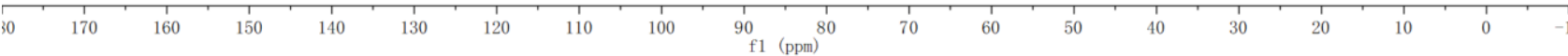




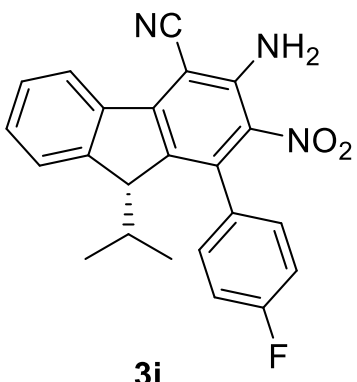

3j

${ }^{19} \mathrm{~F}$ NMR (376 MHz, $\mathrm{CDCl}_{3}$ )
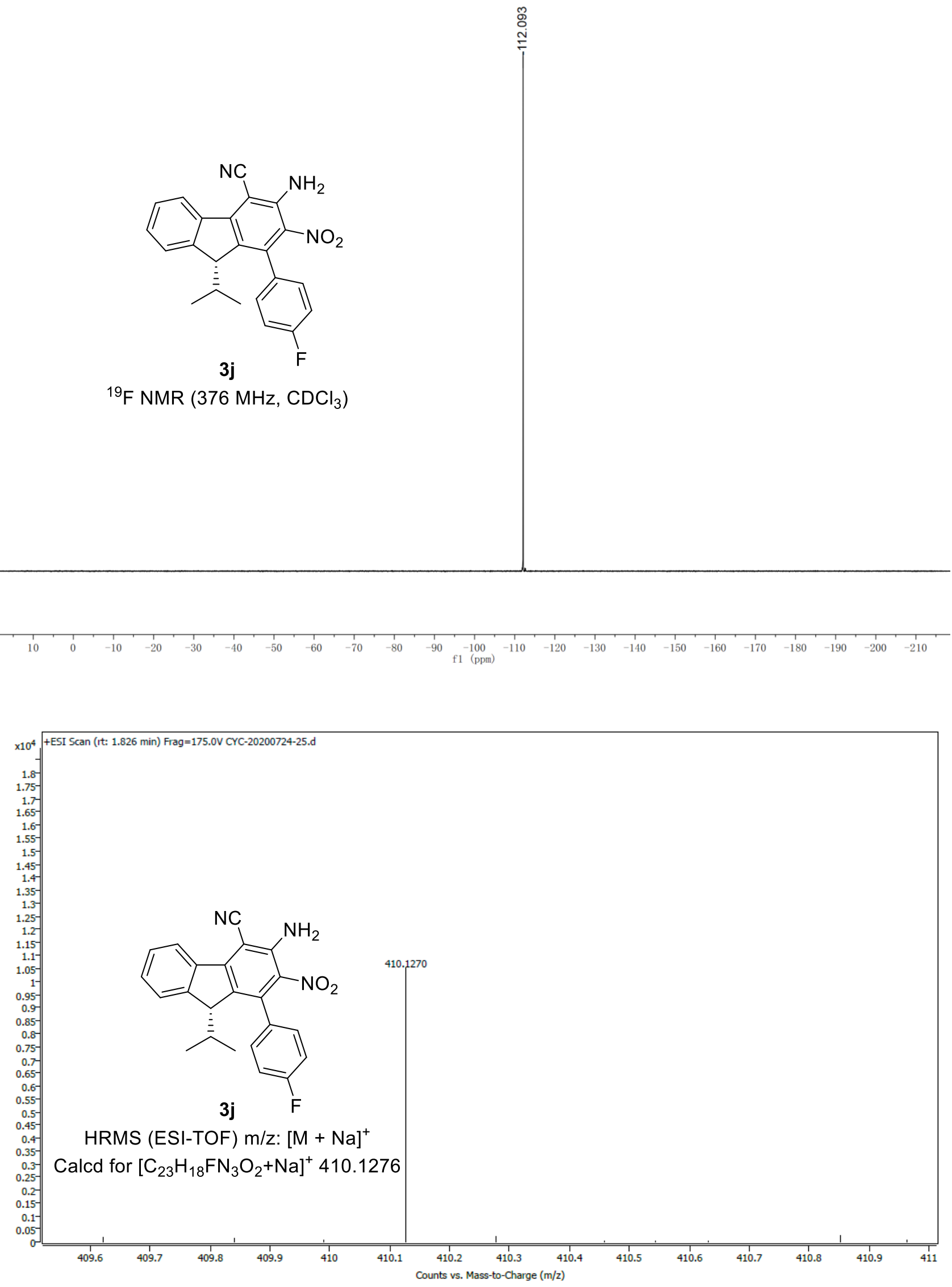

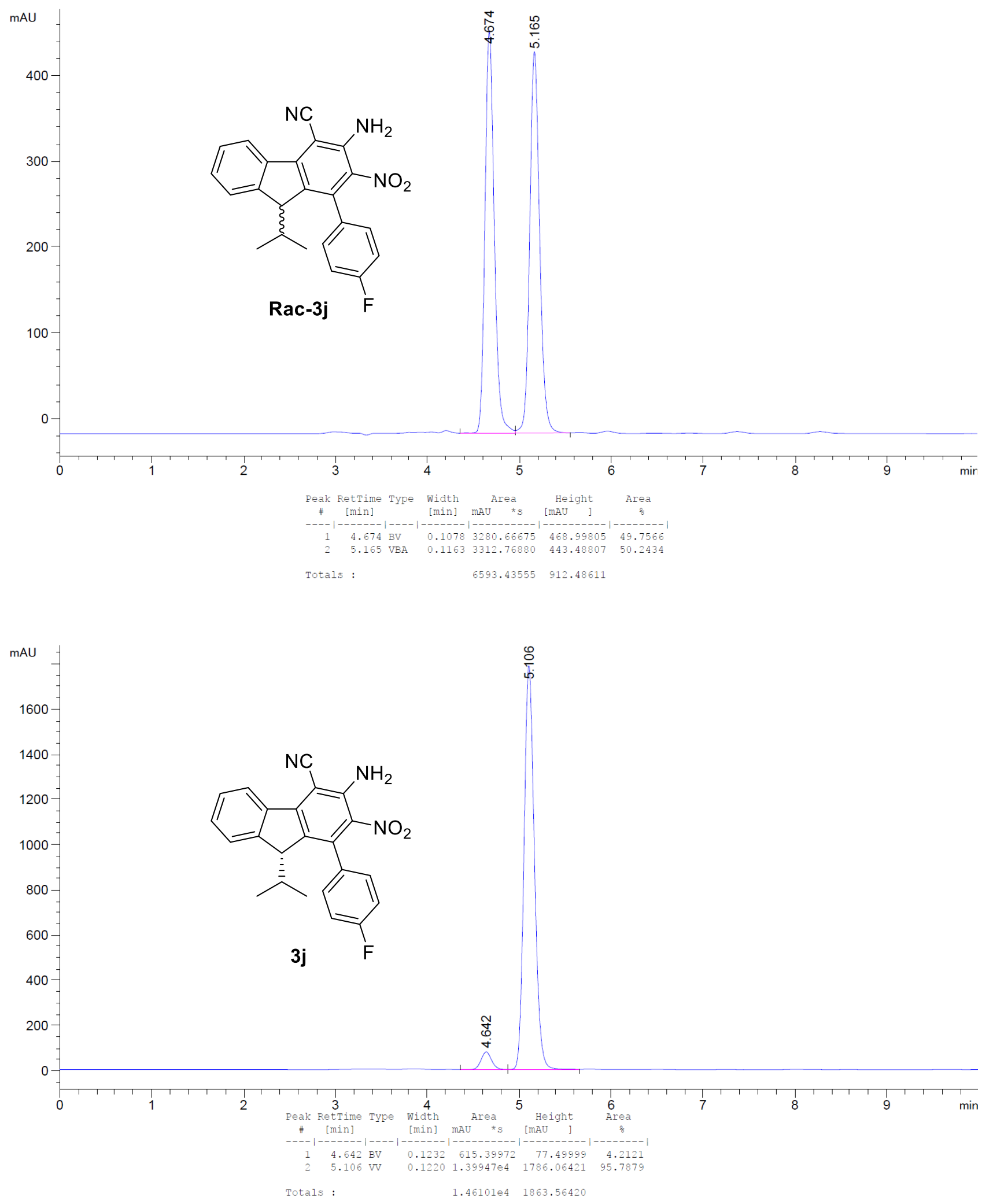


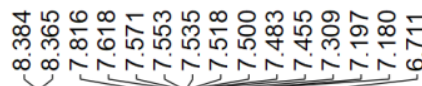

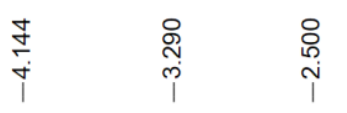

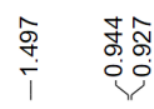

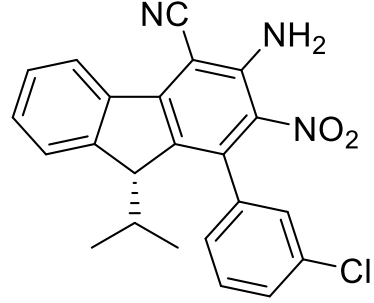

3k

${ }^{1} \mathrm{H}$ NMR (400 MHz, DMSO- $\left.d_{6}\right)$
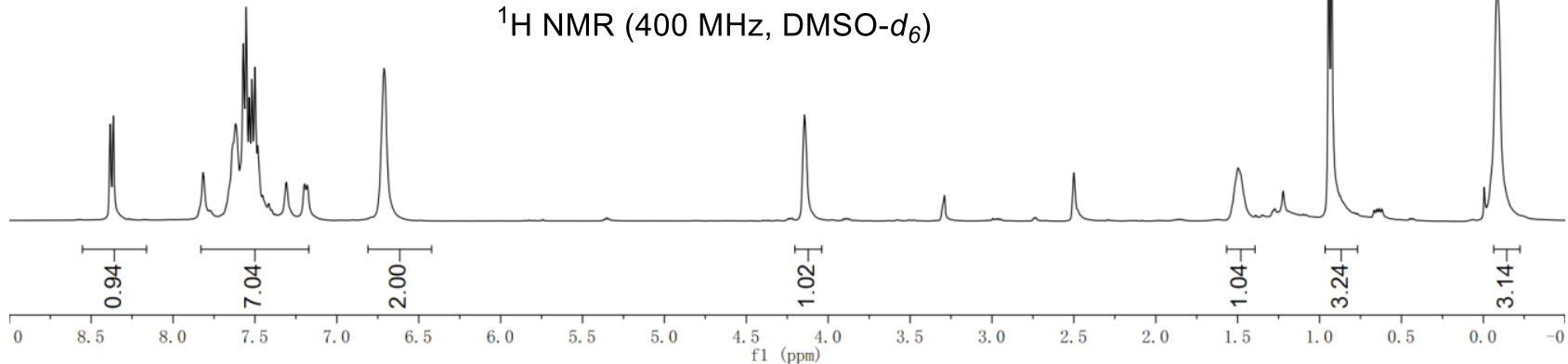

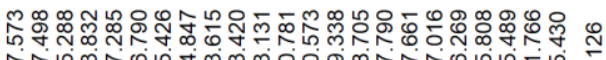

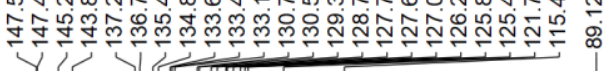

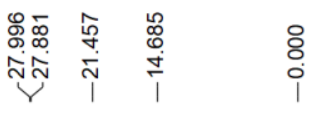<smiles>CC(C)[C@H]1c2ccccc2-c2c(C#N)c(N)c([N+](=O)[O-])c(-c3cccc(Cl)c3)c21</smiles>

3k

${ }^{13} \mathrm{C}$ NMR $\left(100 \mathrm{MHz}\right.$, DMSO- $\left.d_{6}\right)$

160

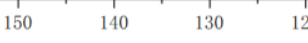

$110 \quad 100$

90

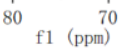



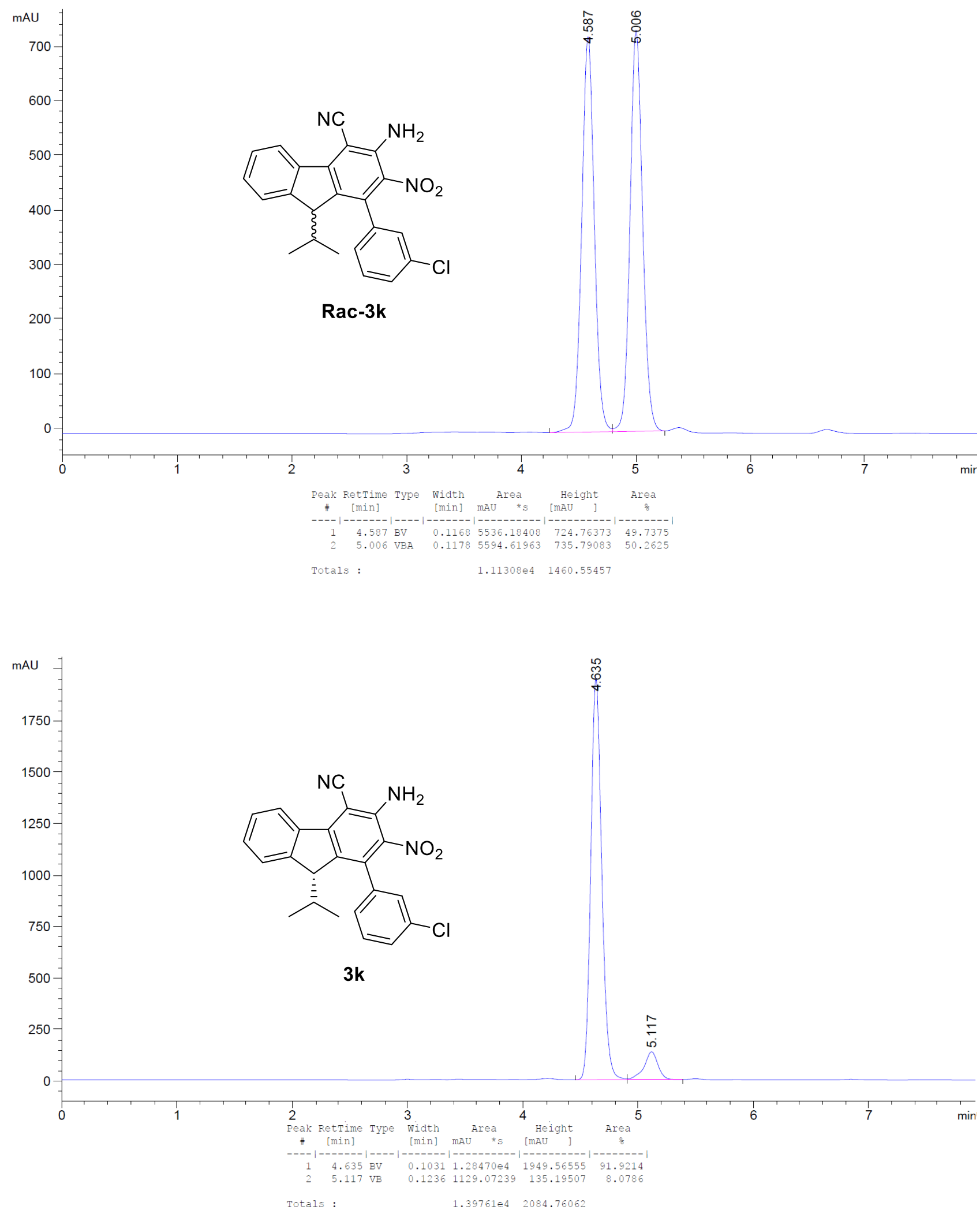


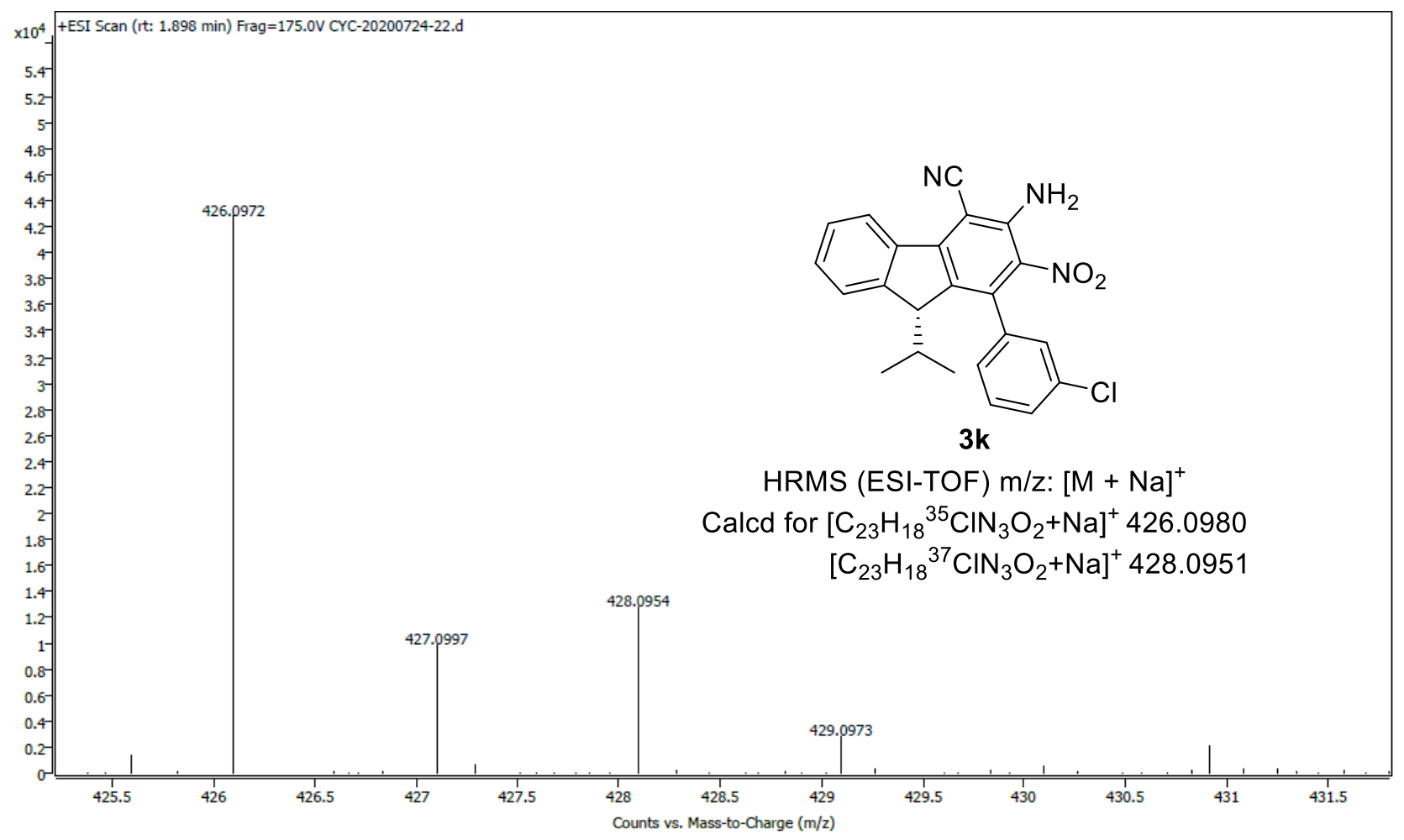



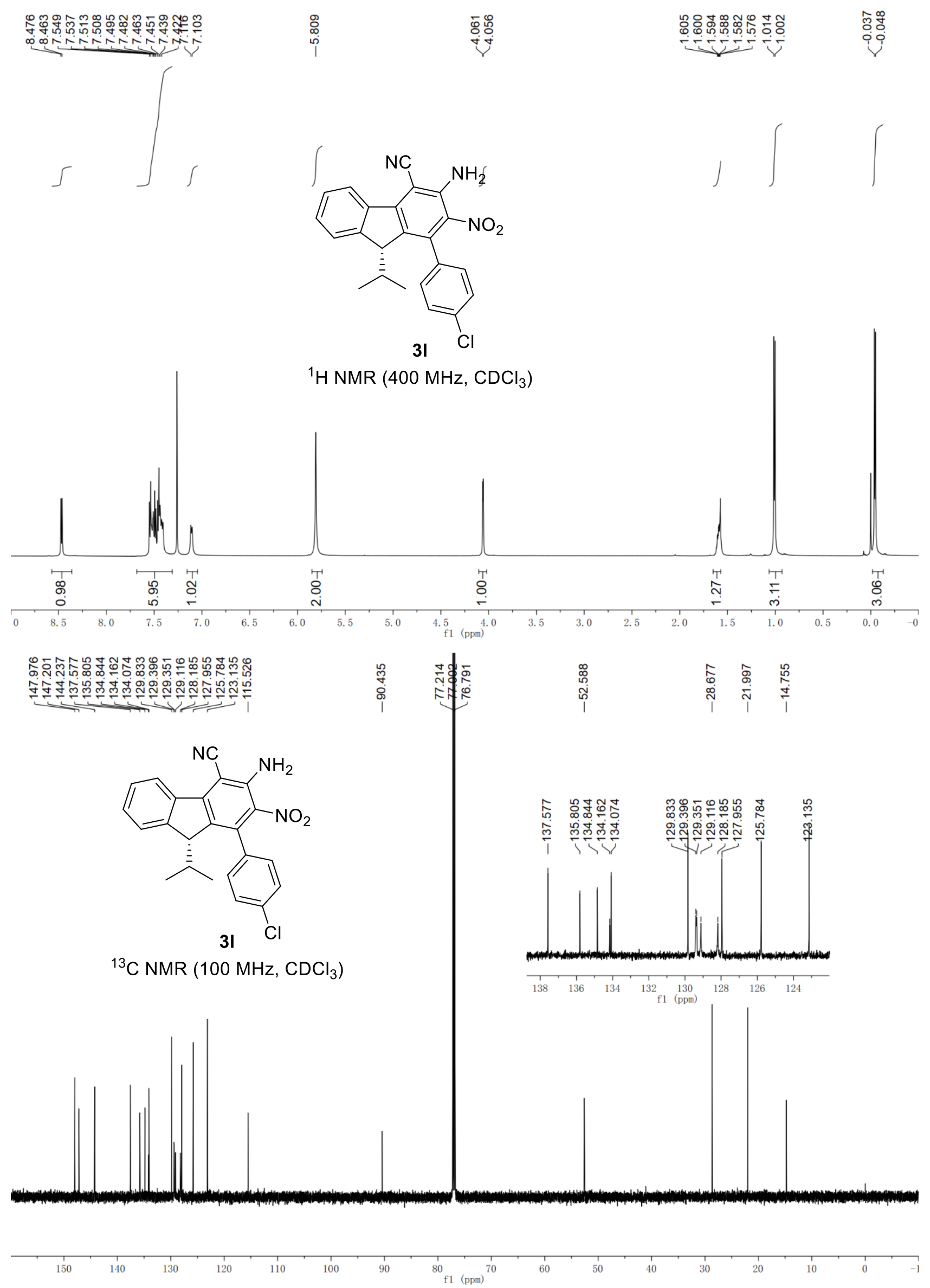

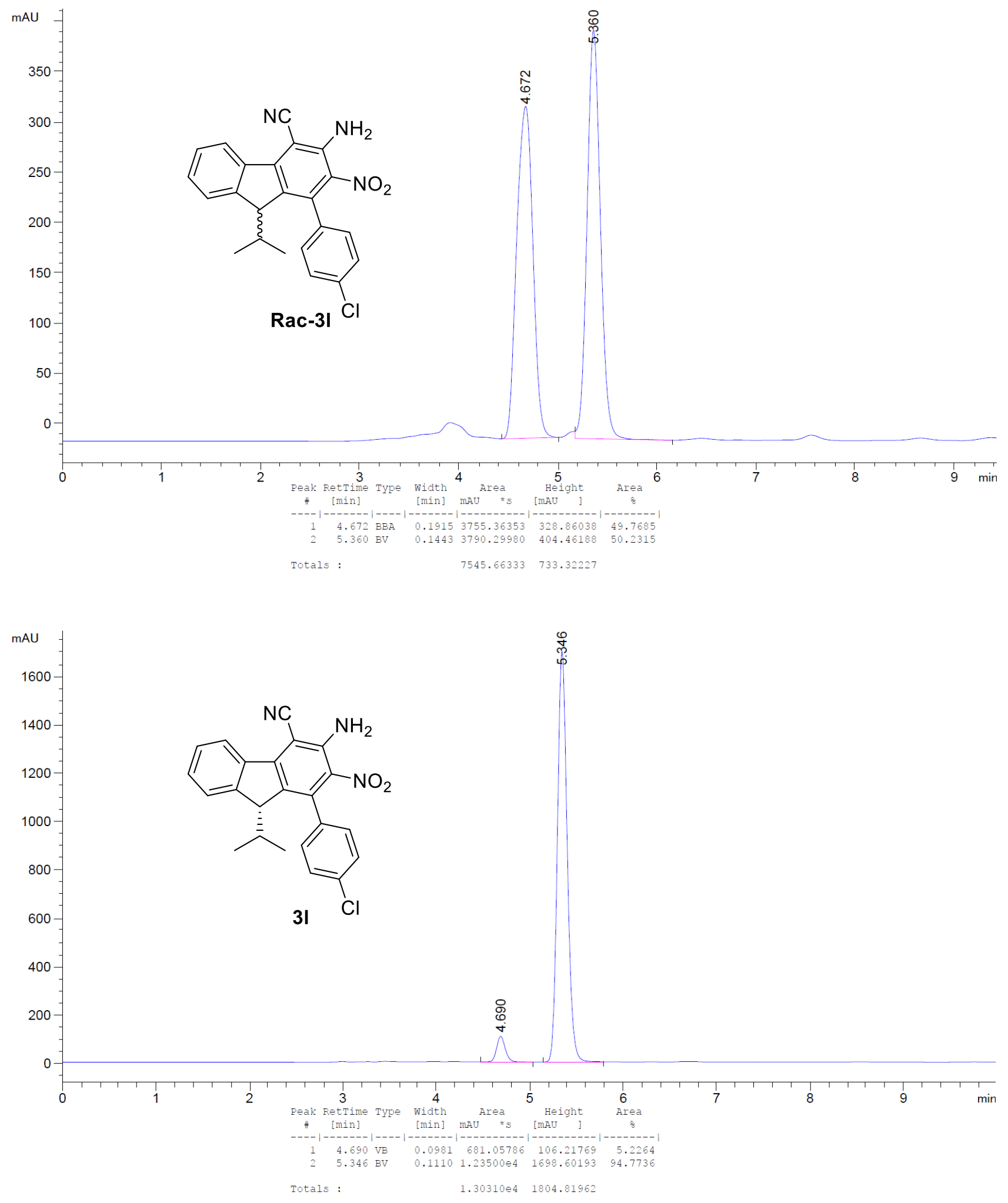


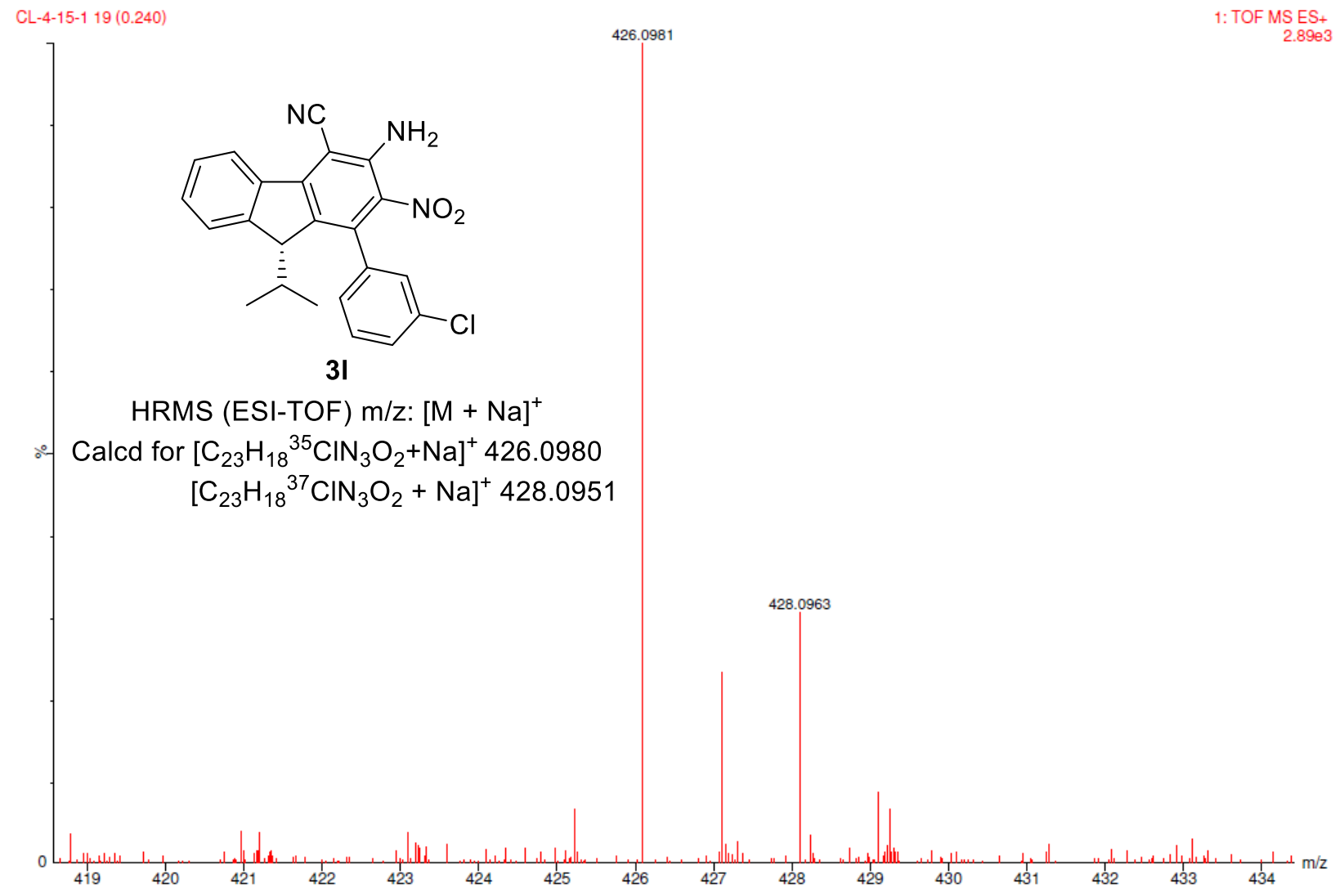




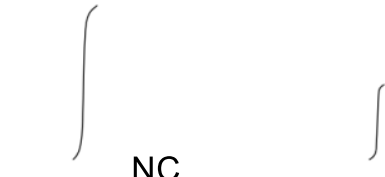
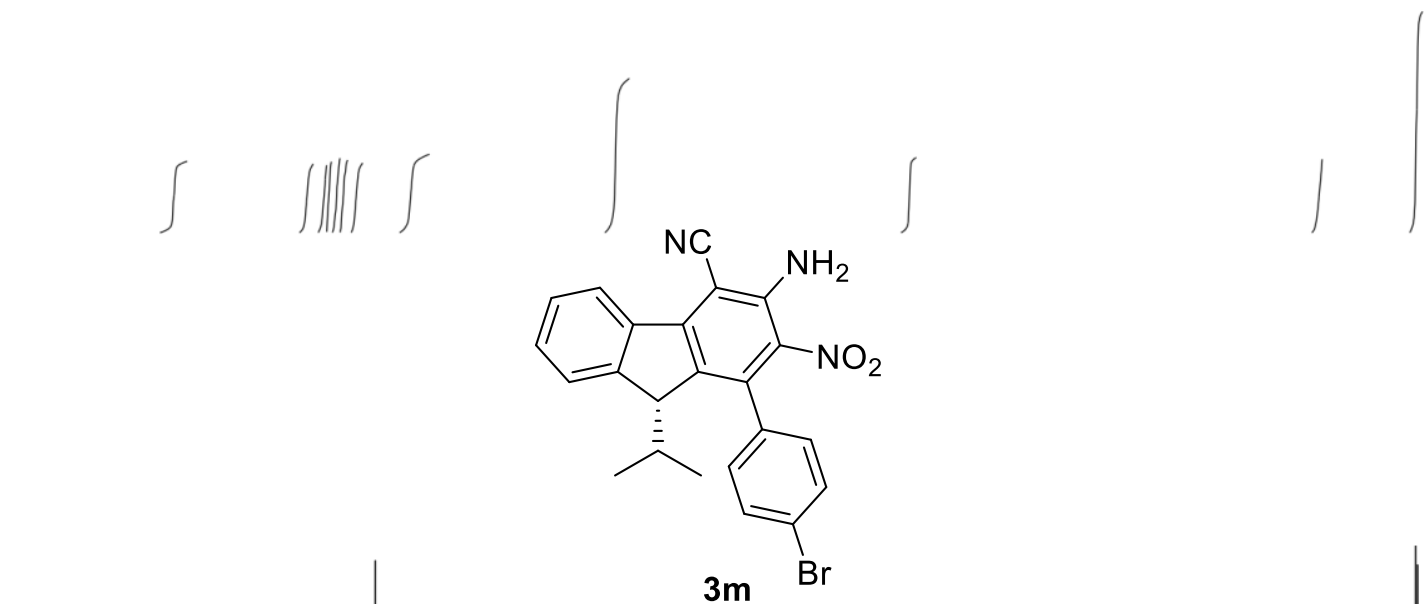

${ }^{1} \mathrm{H}$ NMR $\left(600 \mathrm{MHz}, \mathrm{CDCl}_{3}\right)$

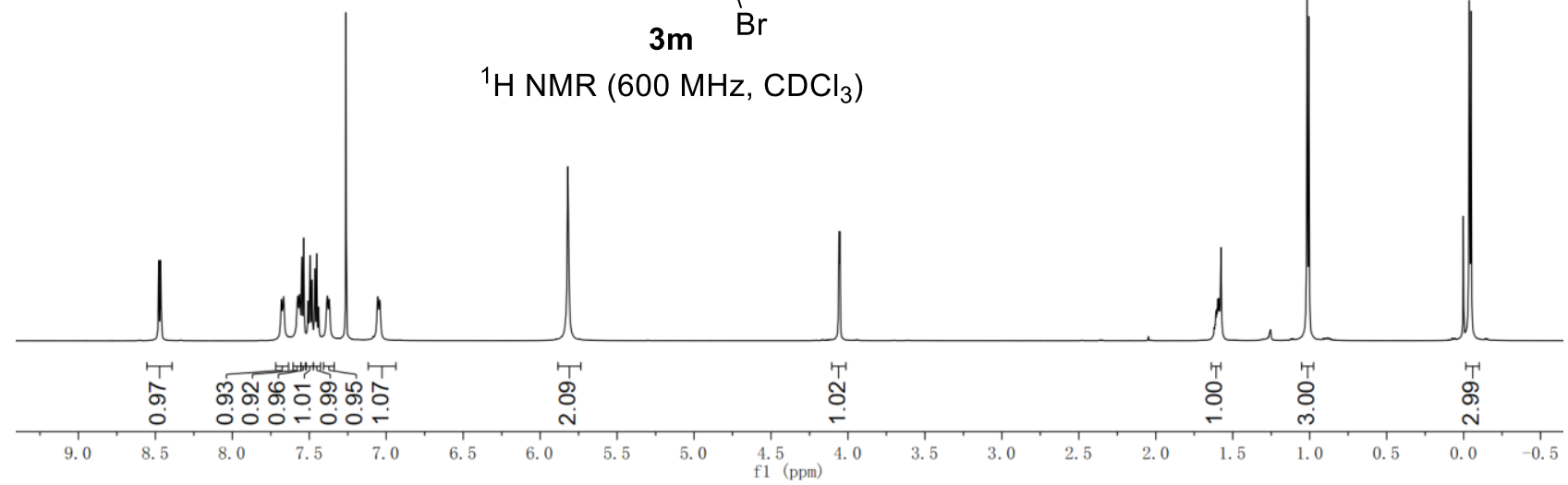

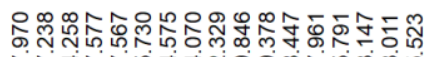

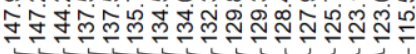<smiles>CC(C)[C@H]1c2ccccc2-c2c(C#N)c(N)c([N+](=O)[O-])c(-c3ccc(Br)cc3)c21</smiles>

${ }^{13} \mathrm{C}$ NMR $\left(150 \mathrm{MHz}, \mathrm{CDCl}_{3}\right)$

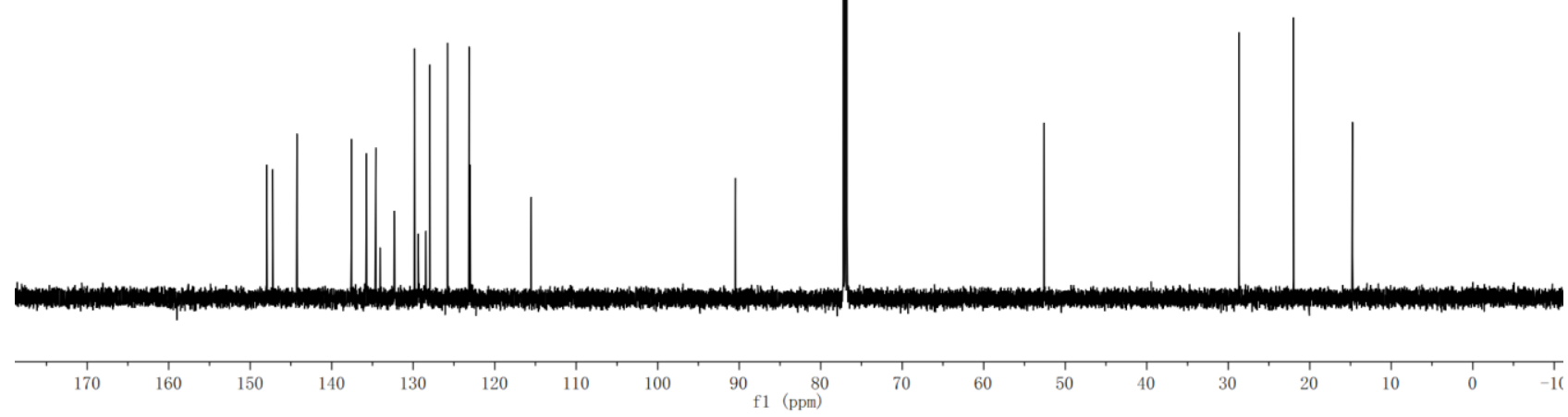



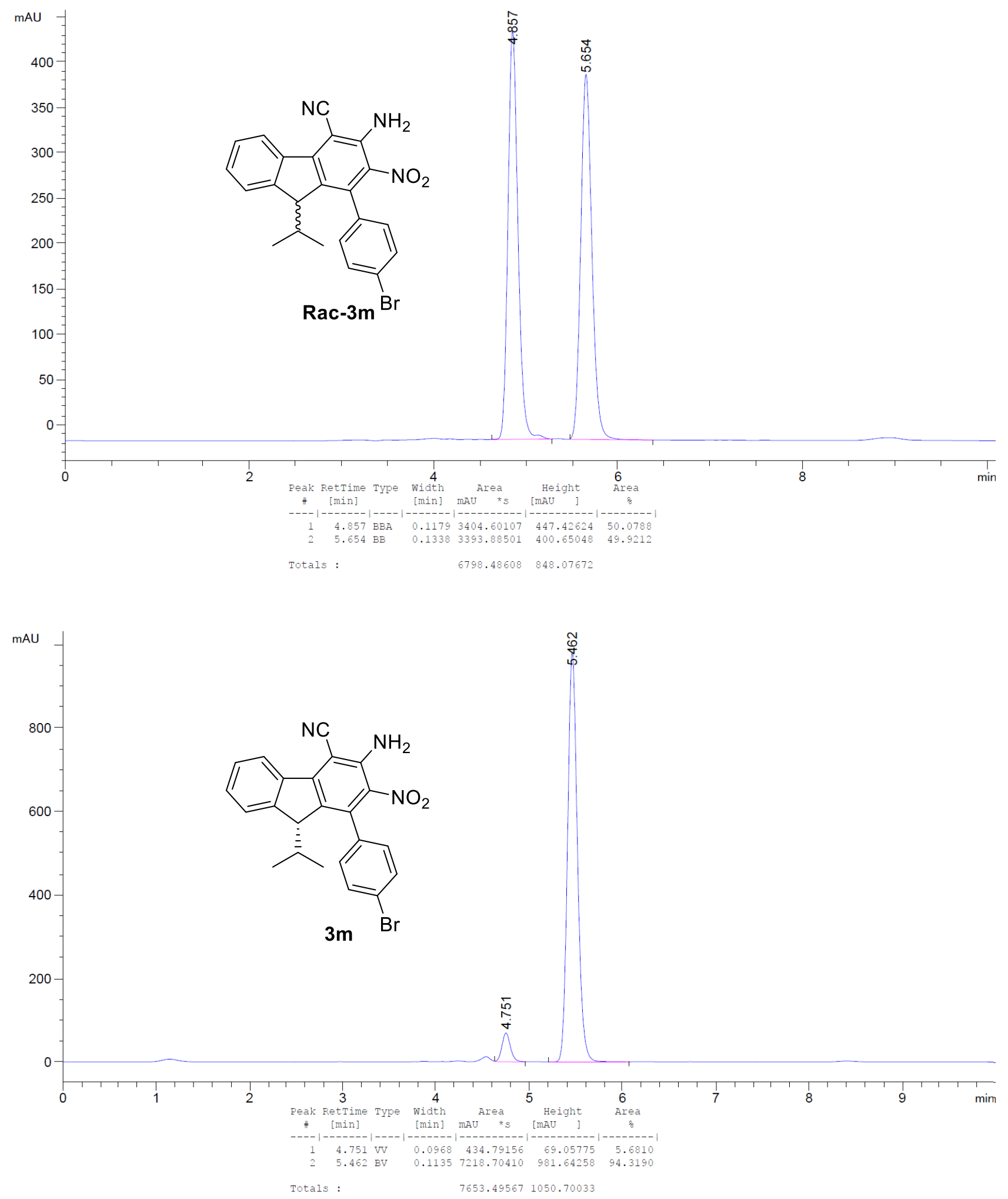


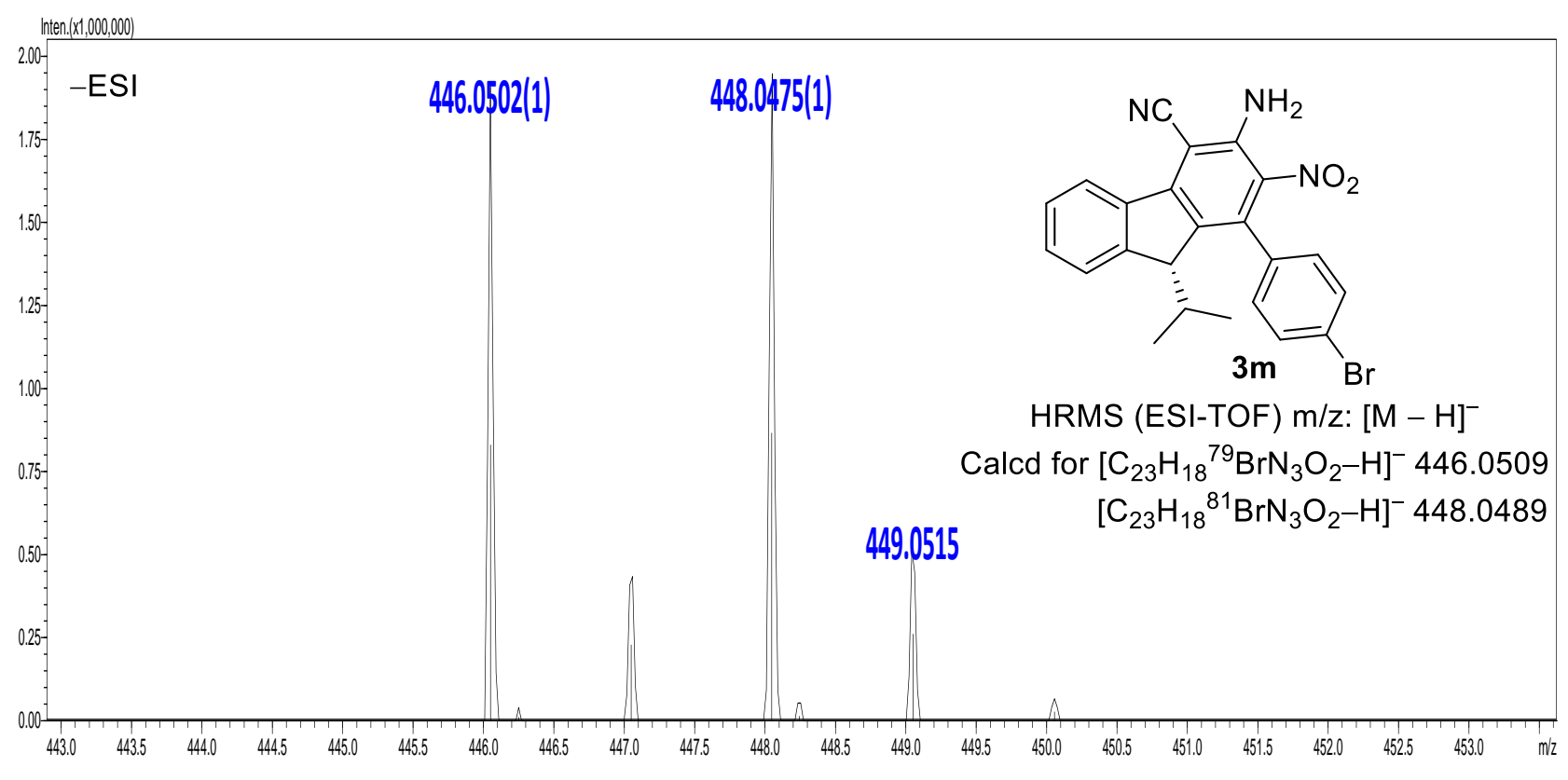



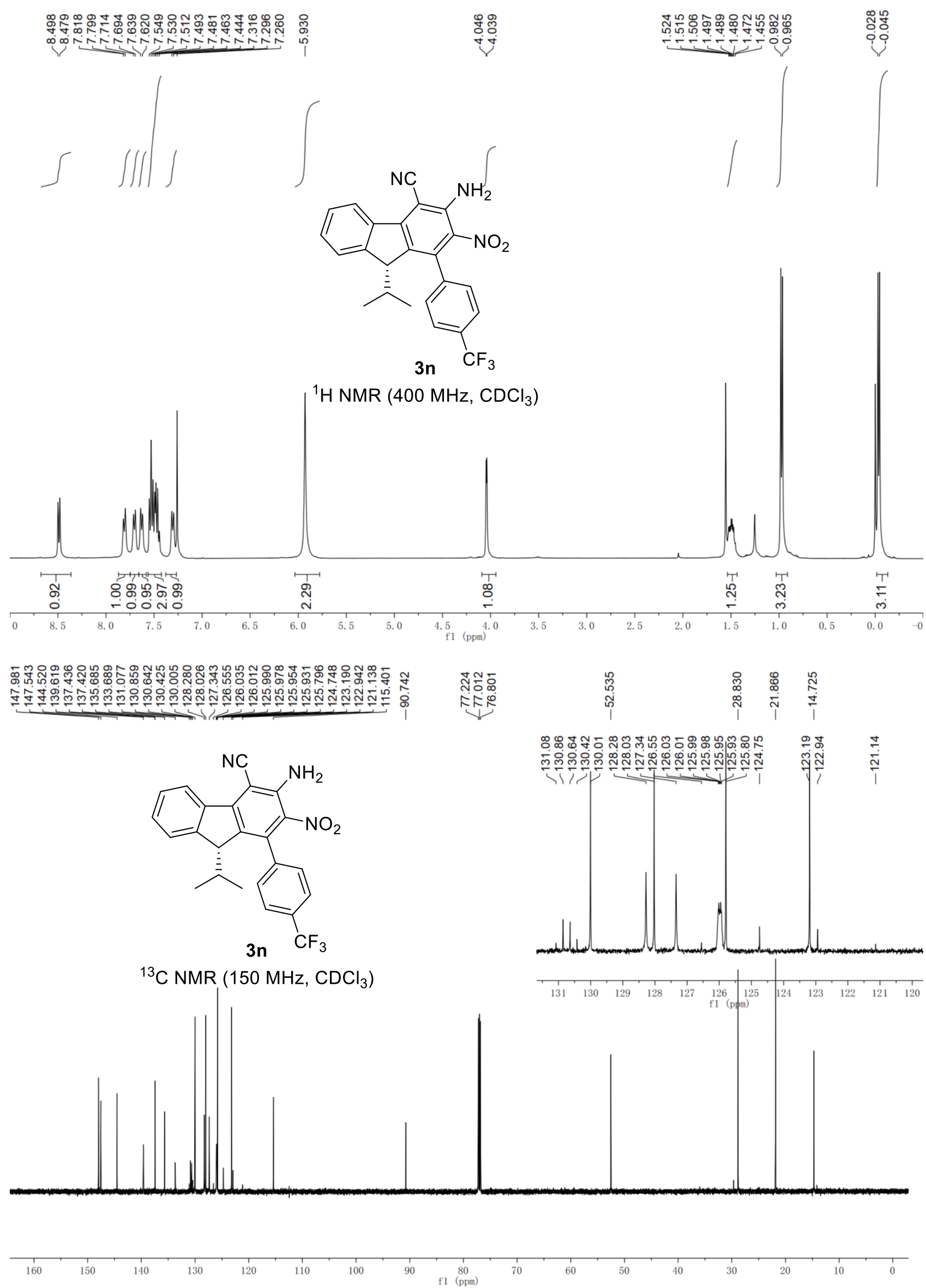


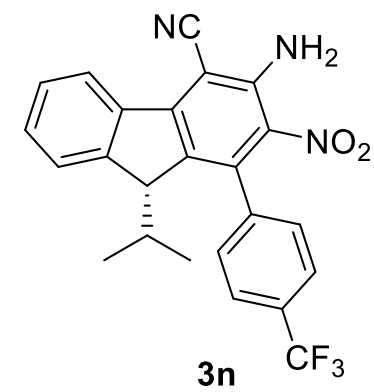

${ }^{19} \mathrm{~F} \mathrm{NMR} \mathrm{(376} \mathrm{MHz,} \mathrm{CDCl}_{3}$ )
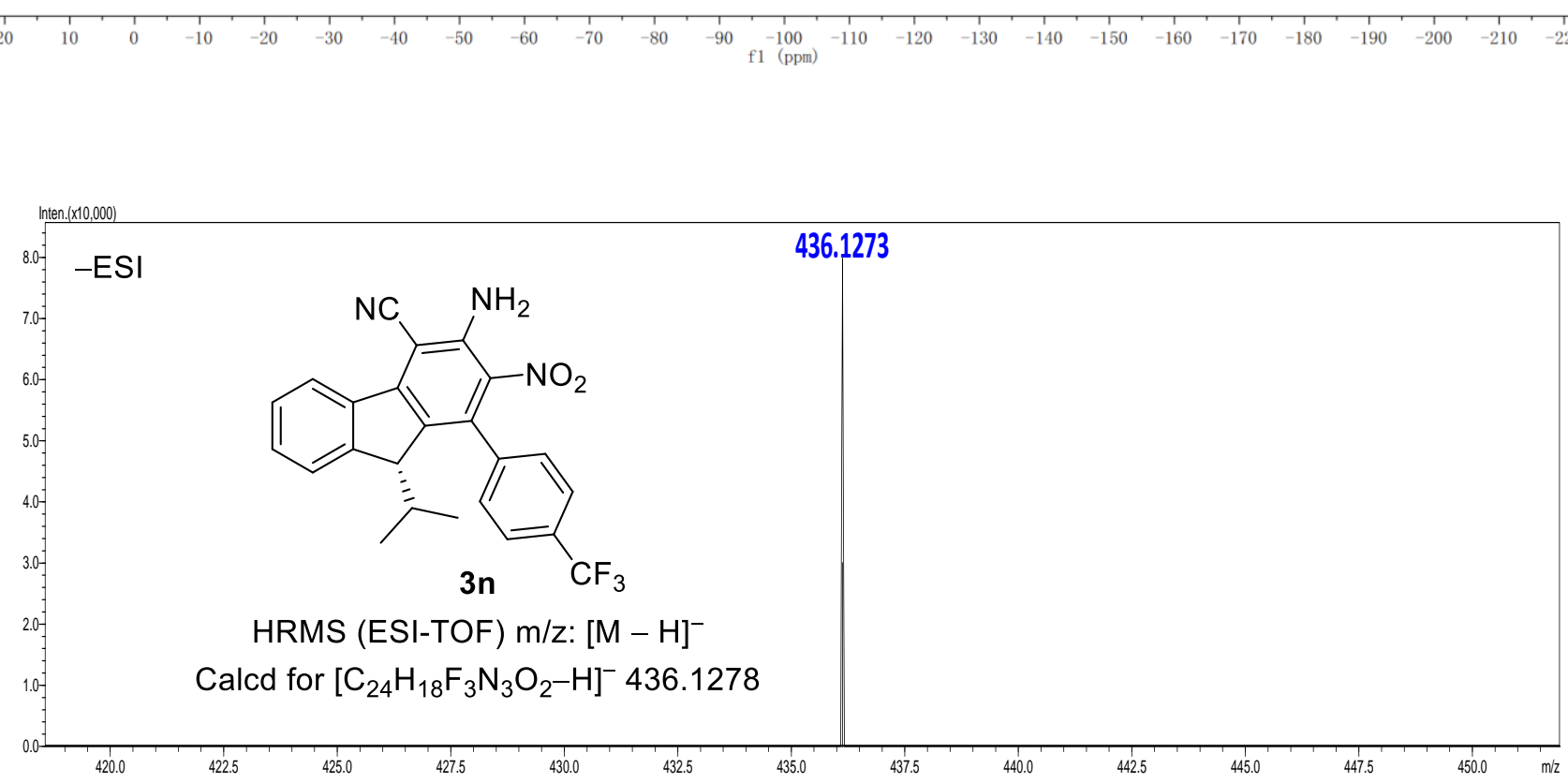

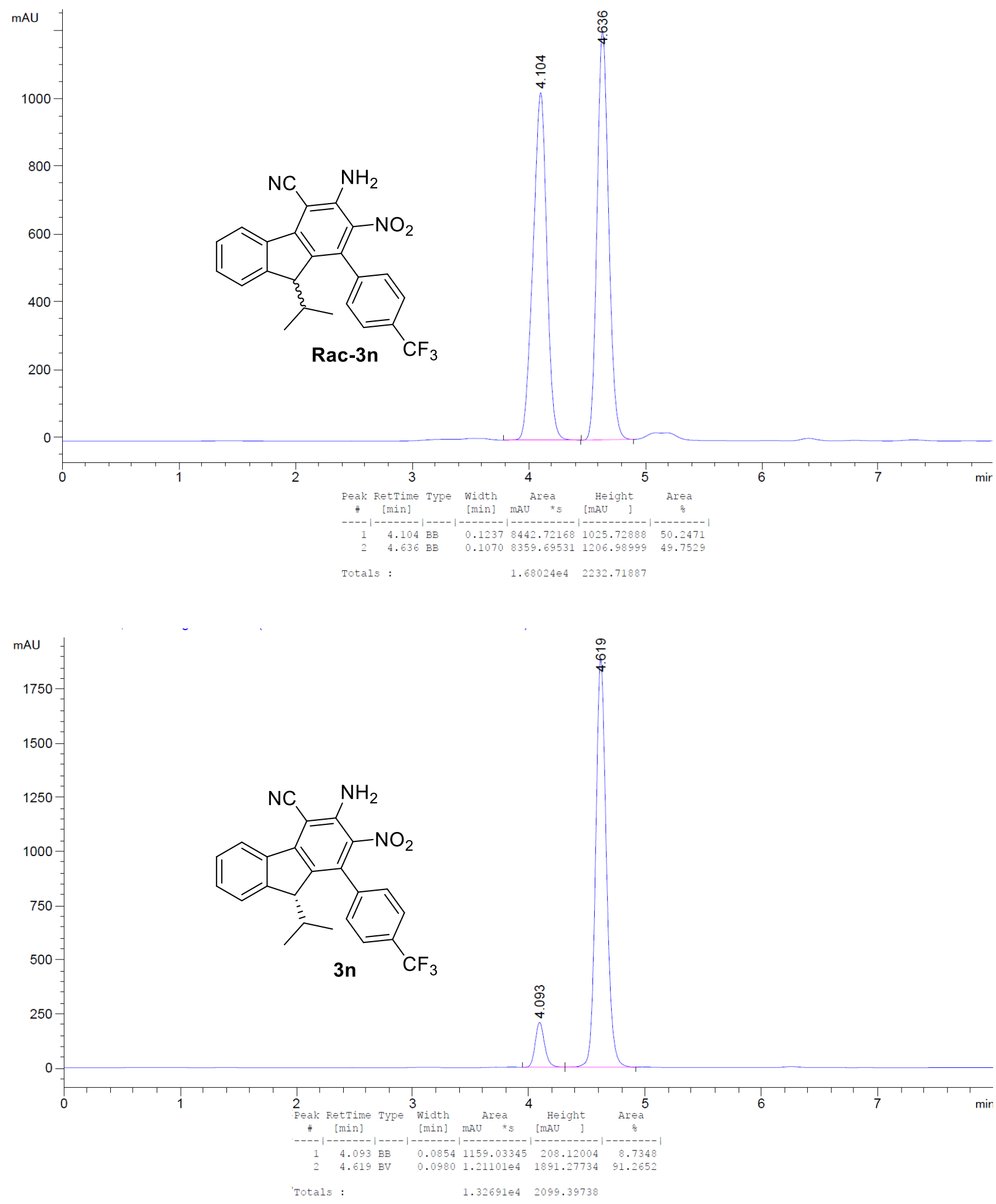

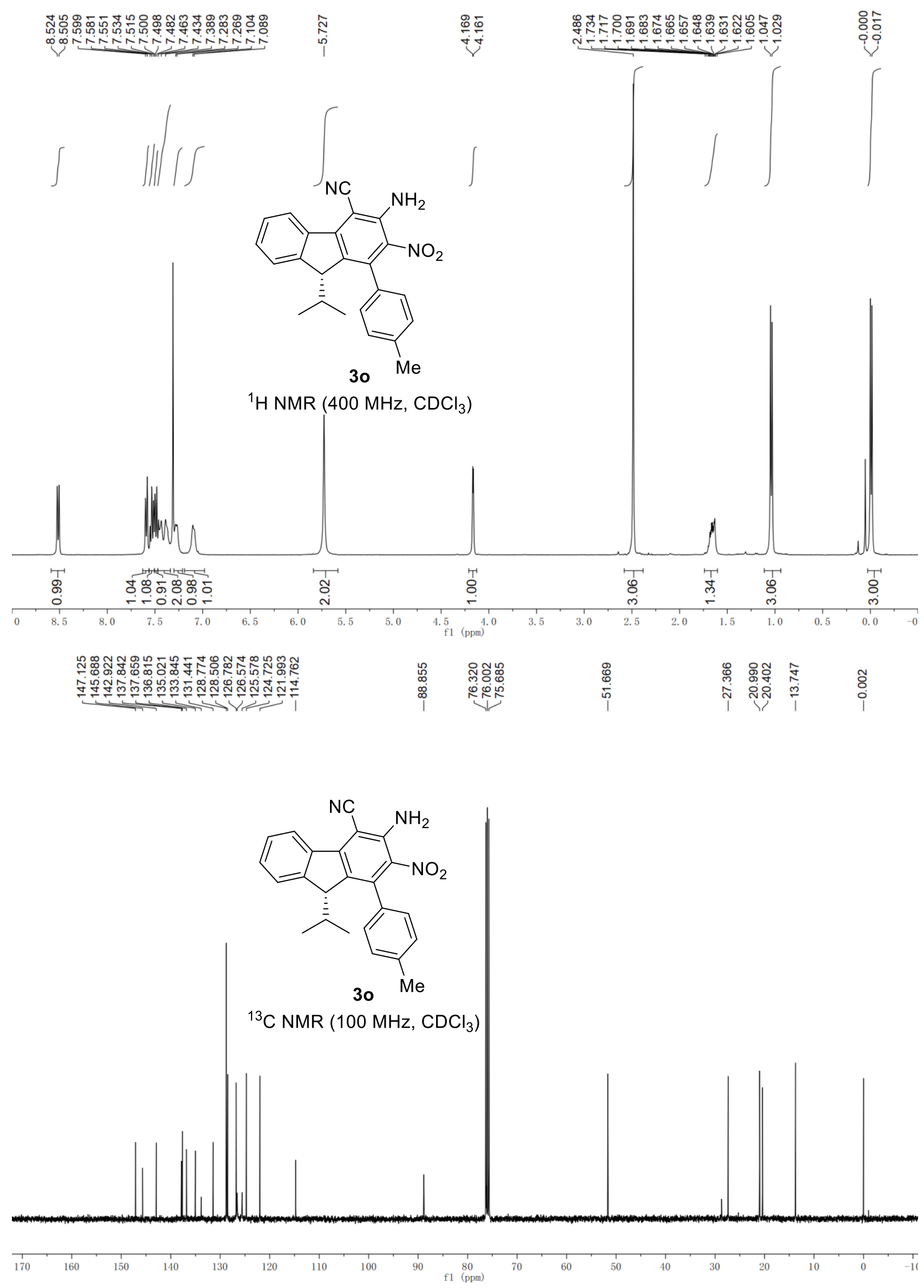

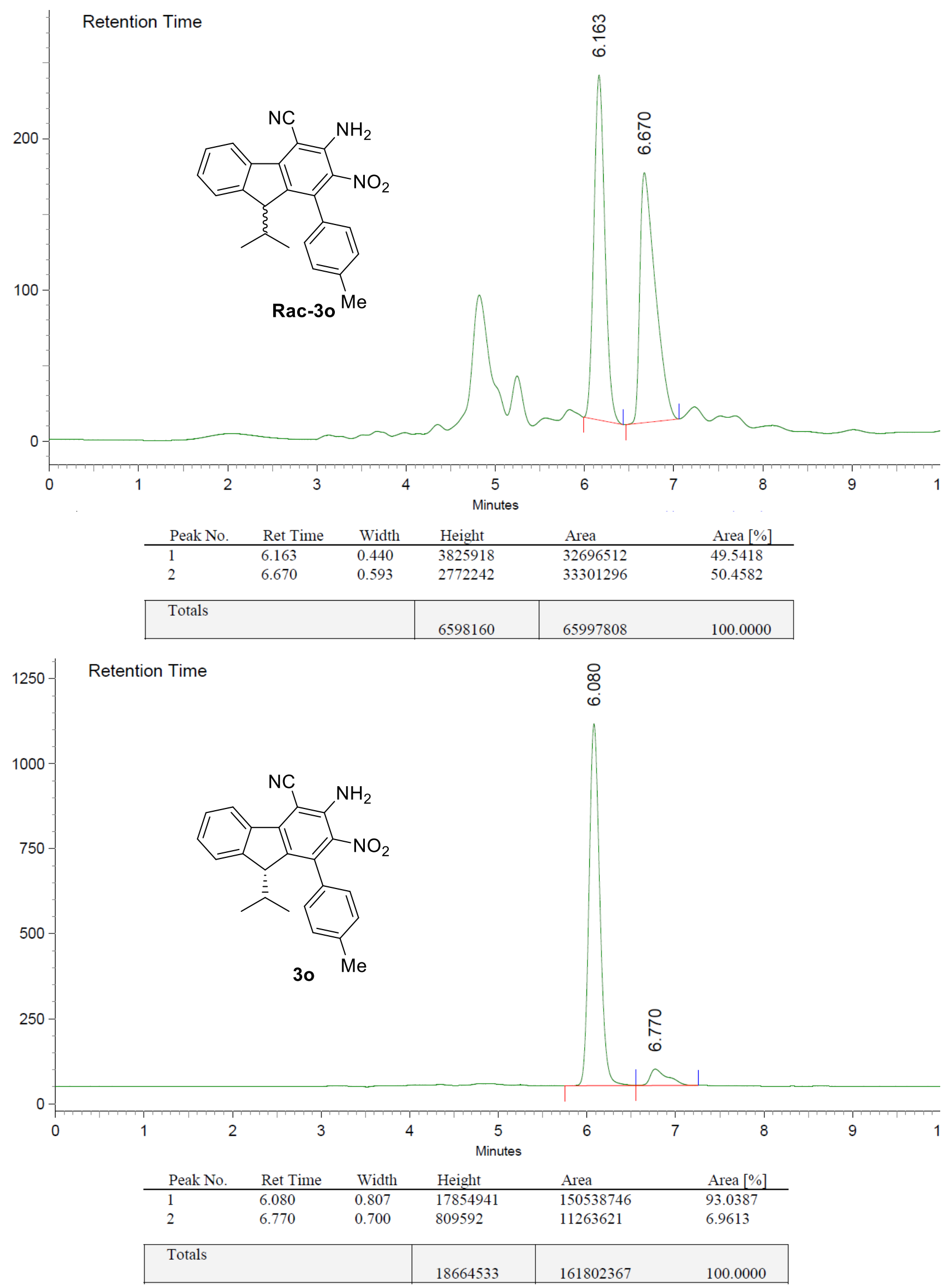


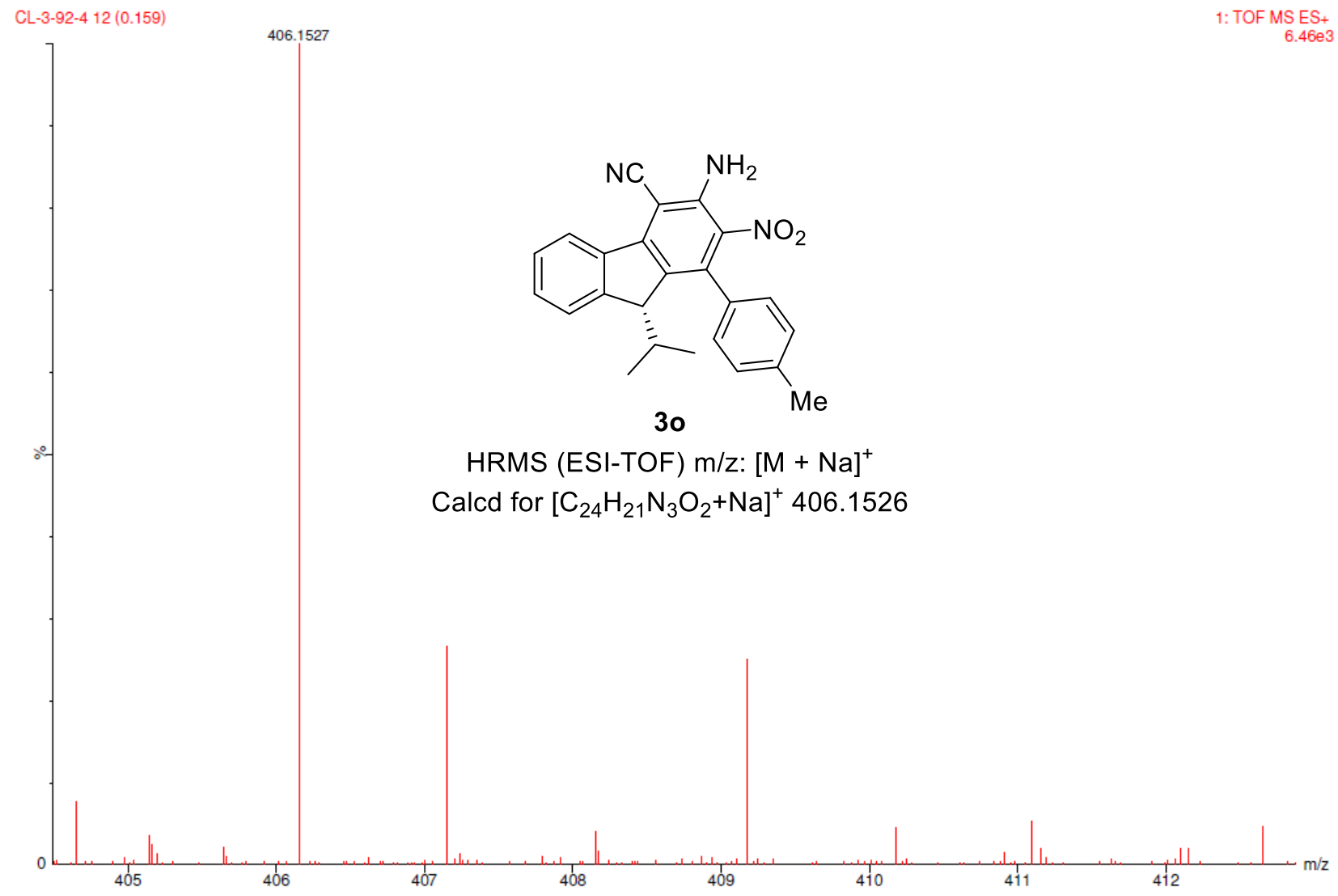




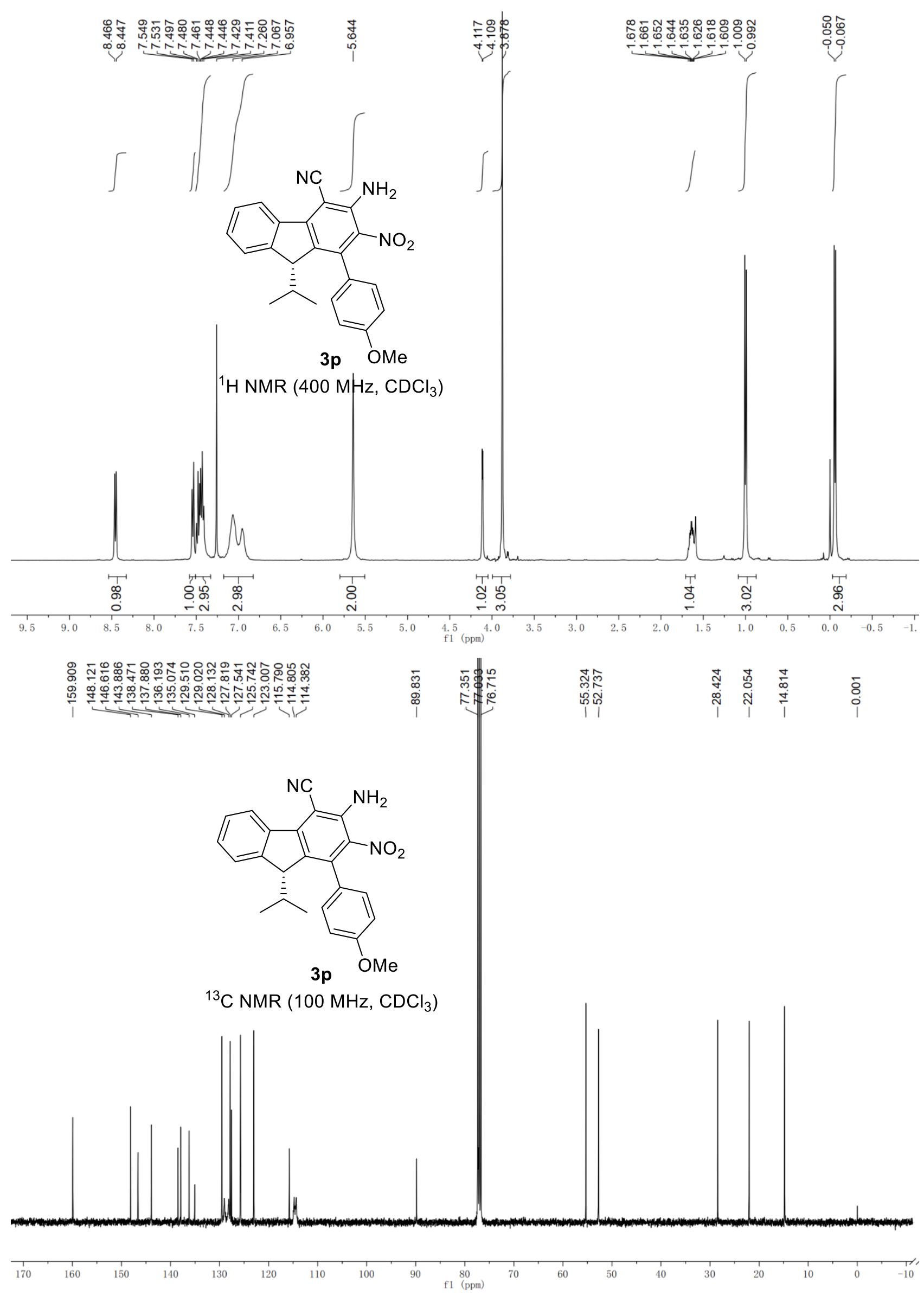



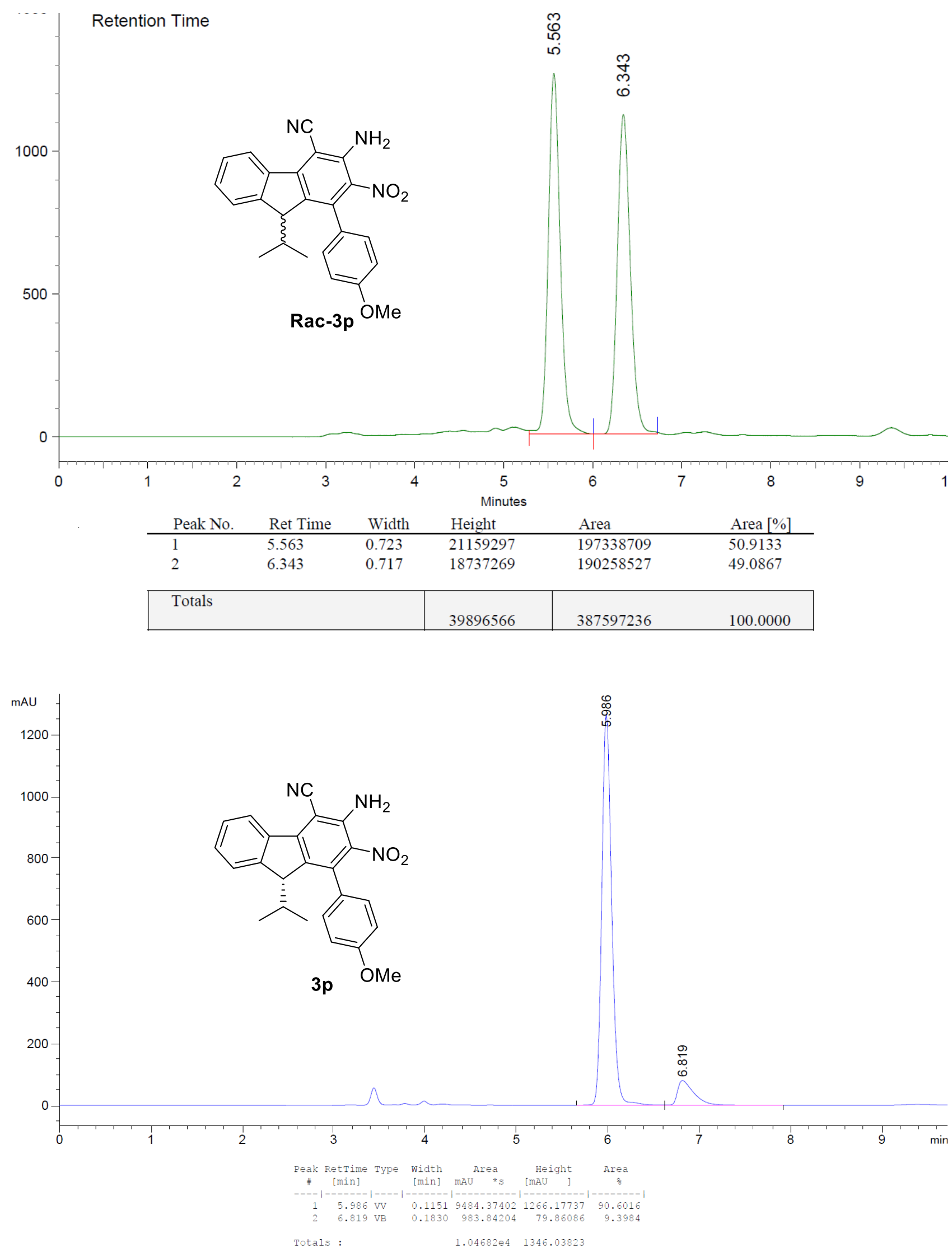


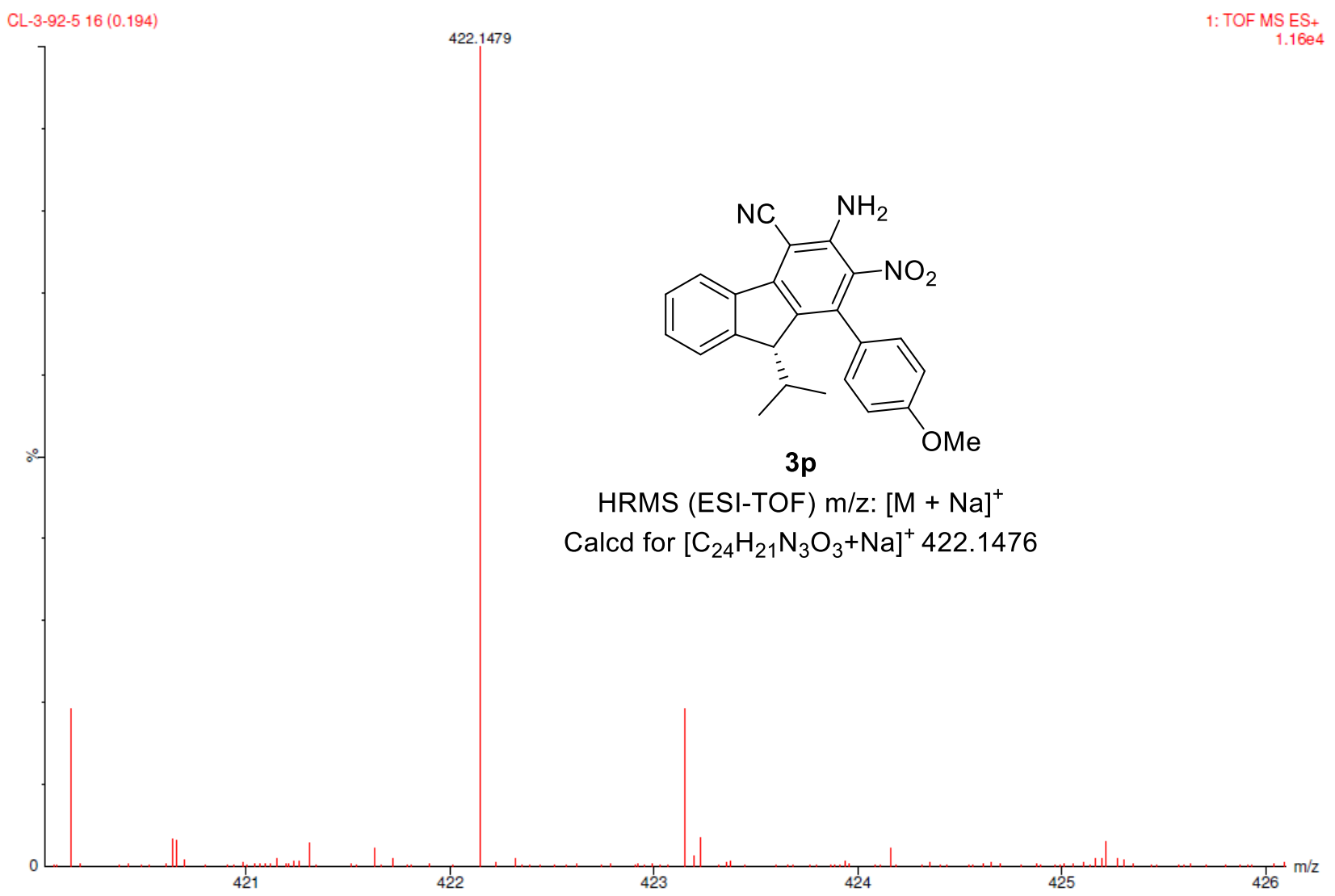



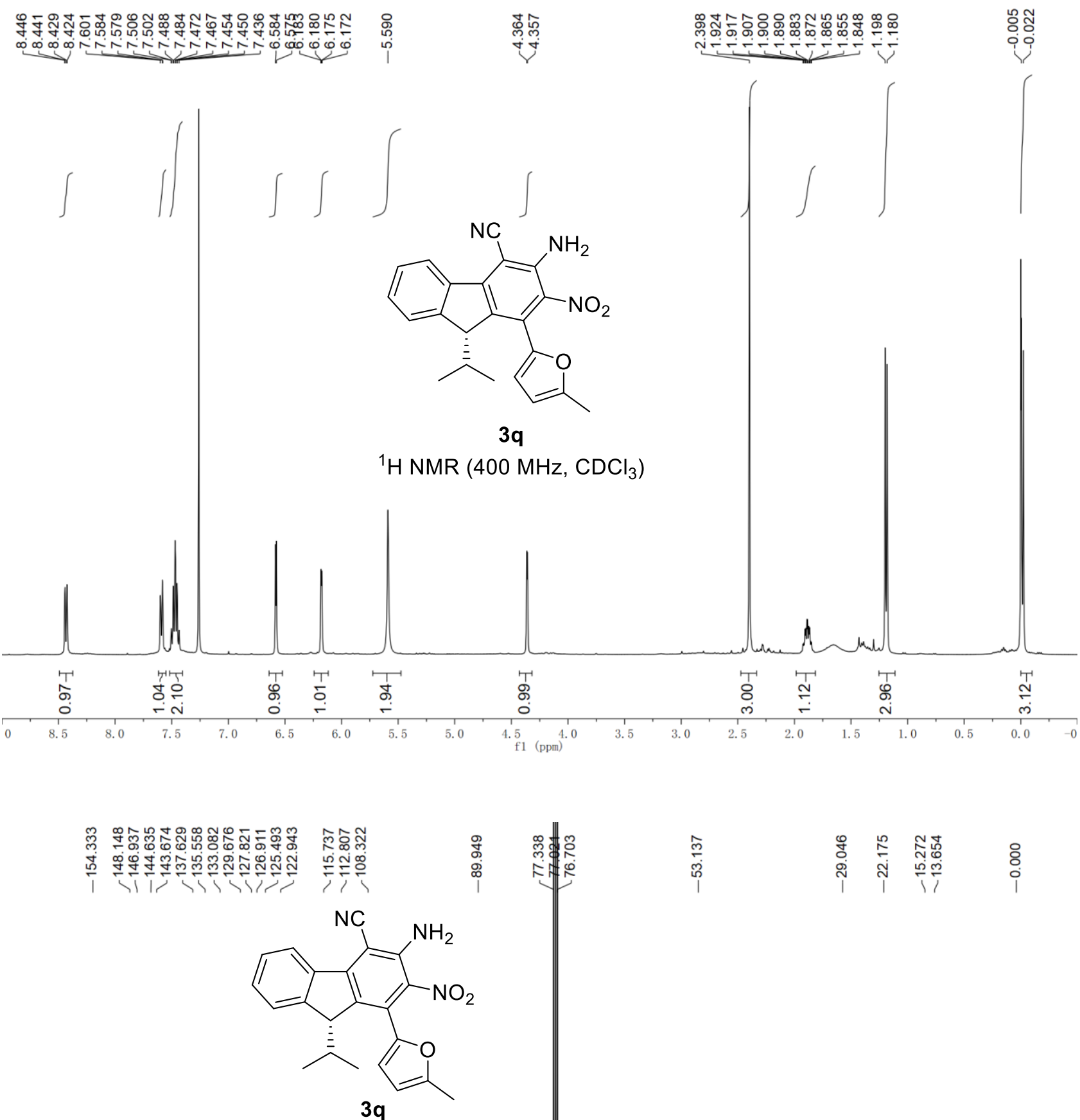

${ }^{13} \mathrm{C}$ NMR $\left(100 \mathrm{MHz}, \mathrm{CDCl}_{3}\right)$

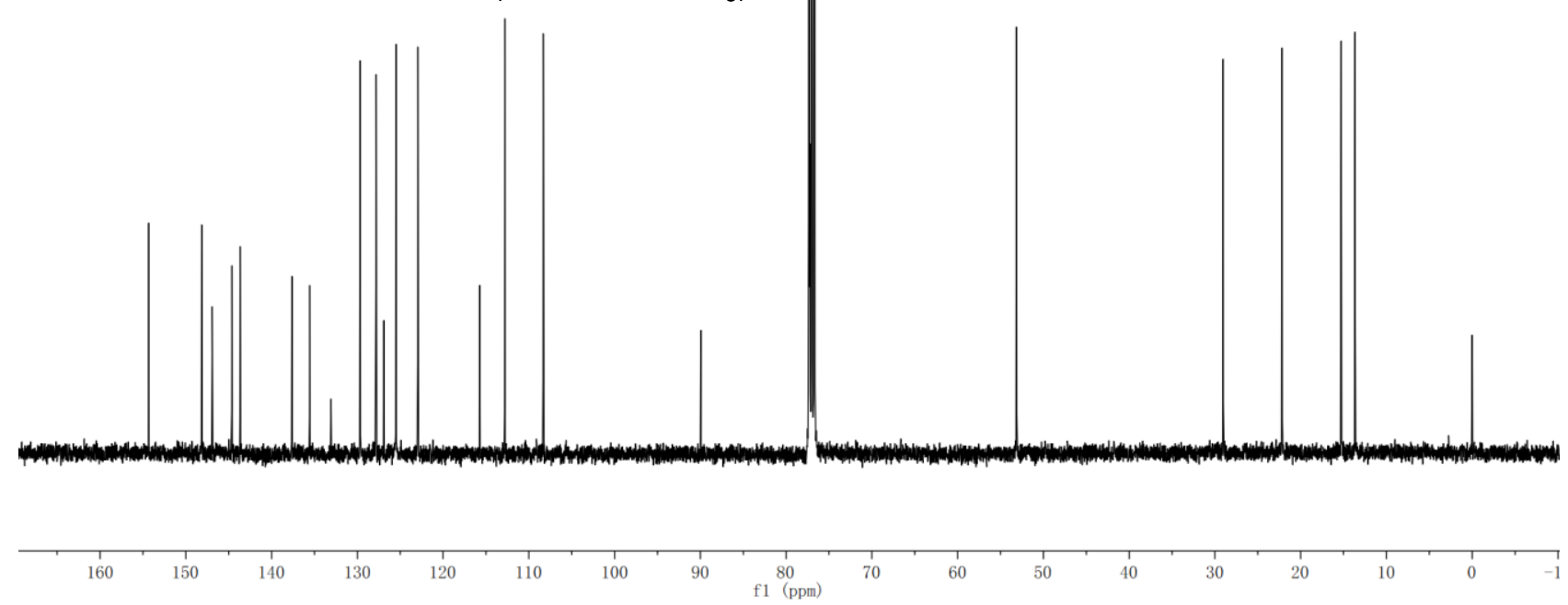



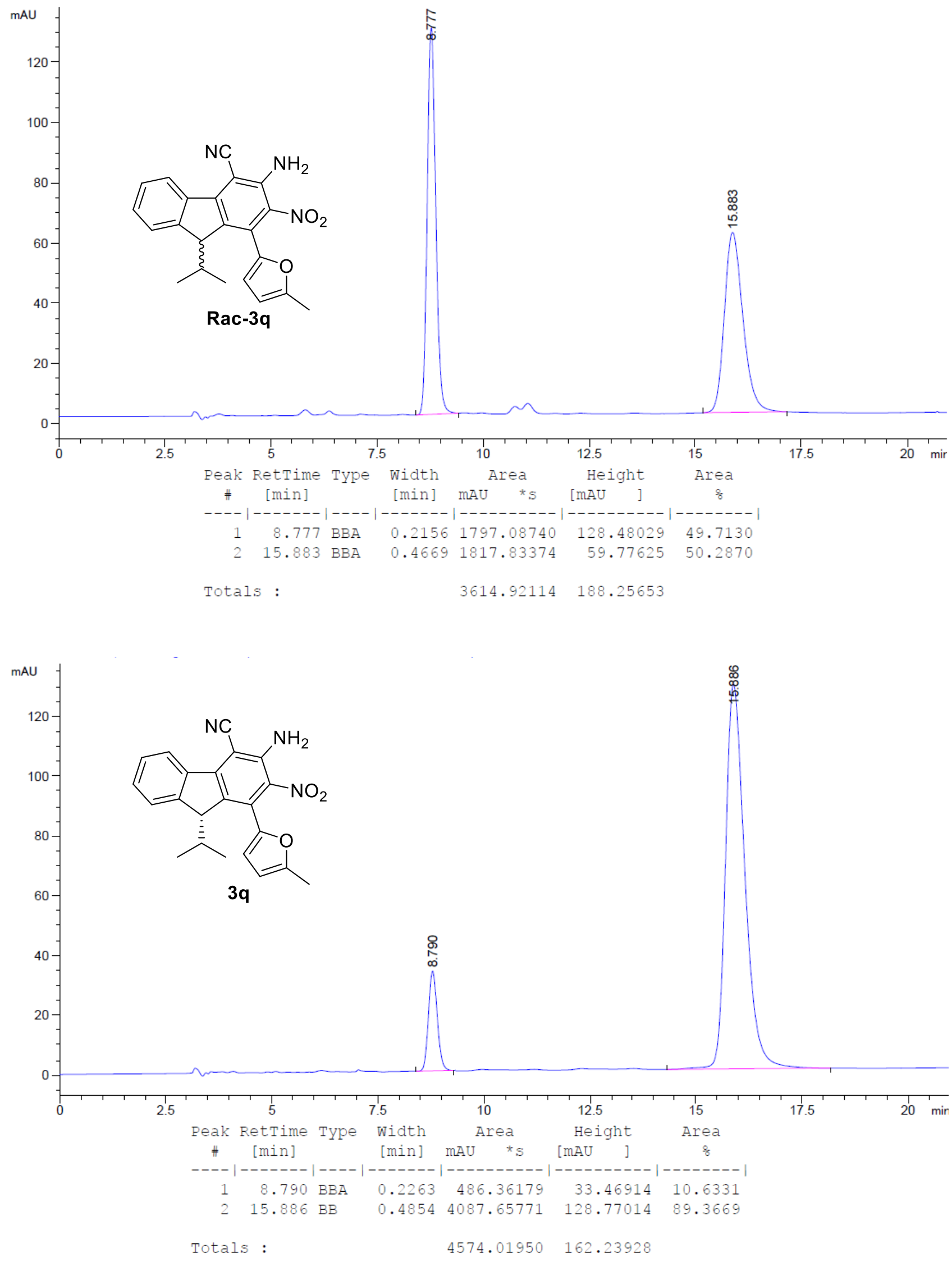


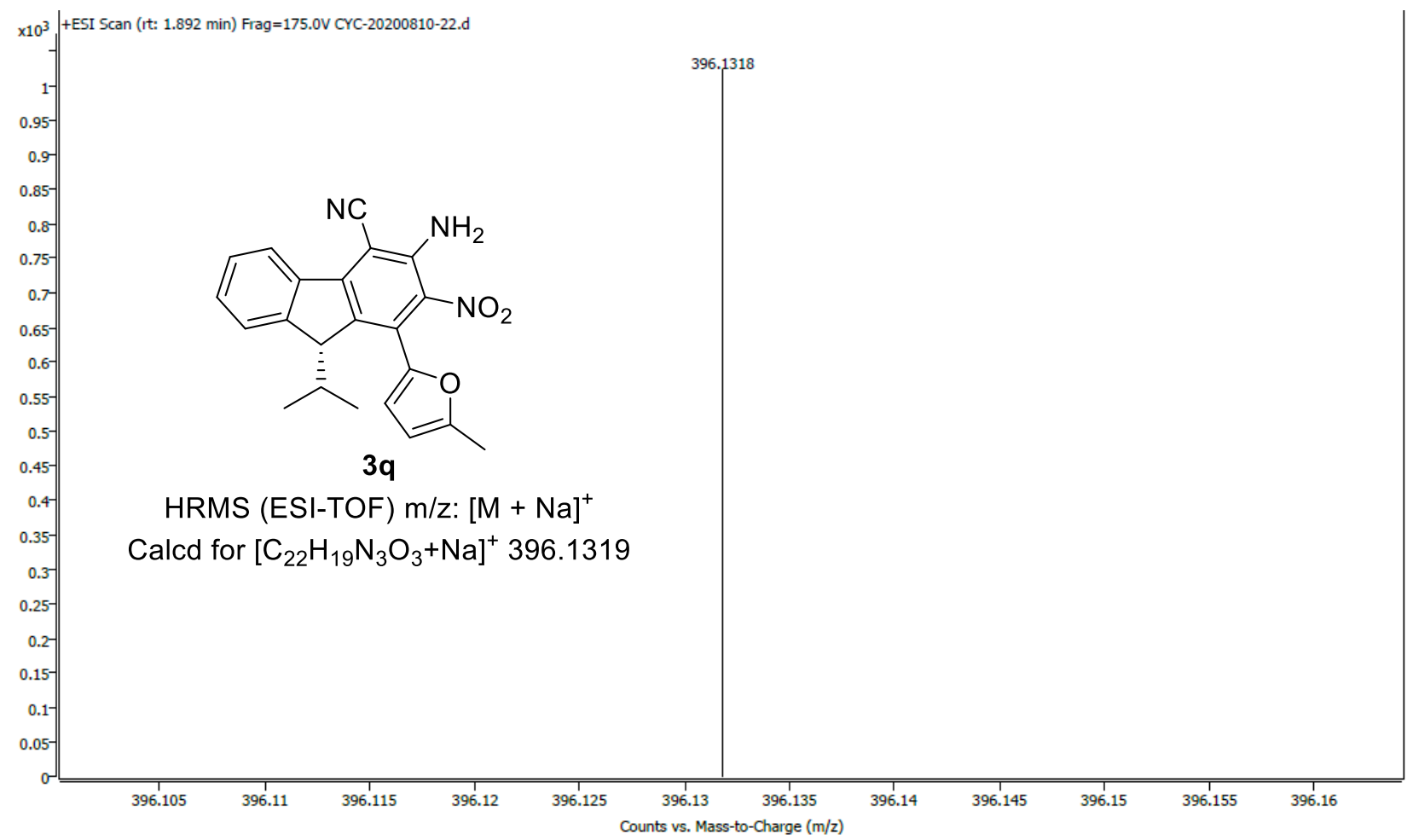




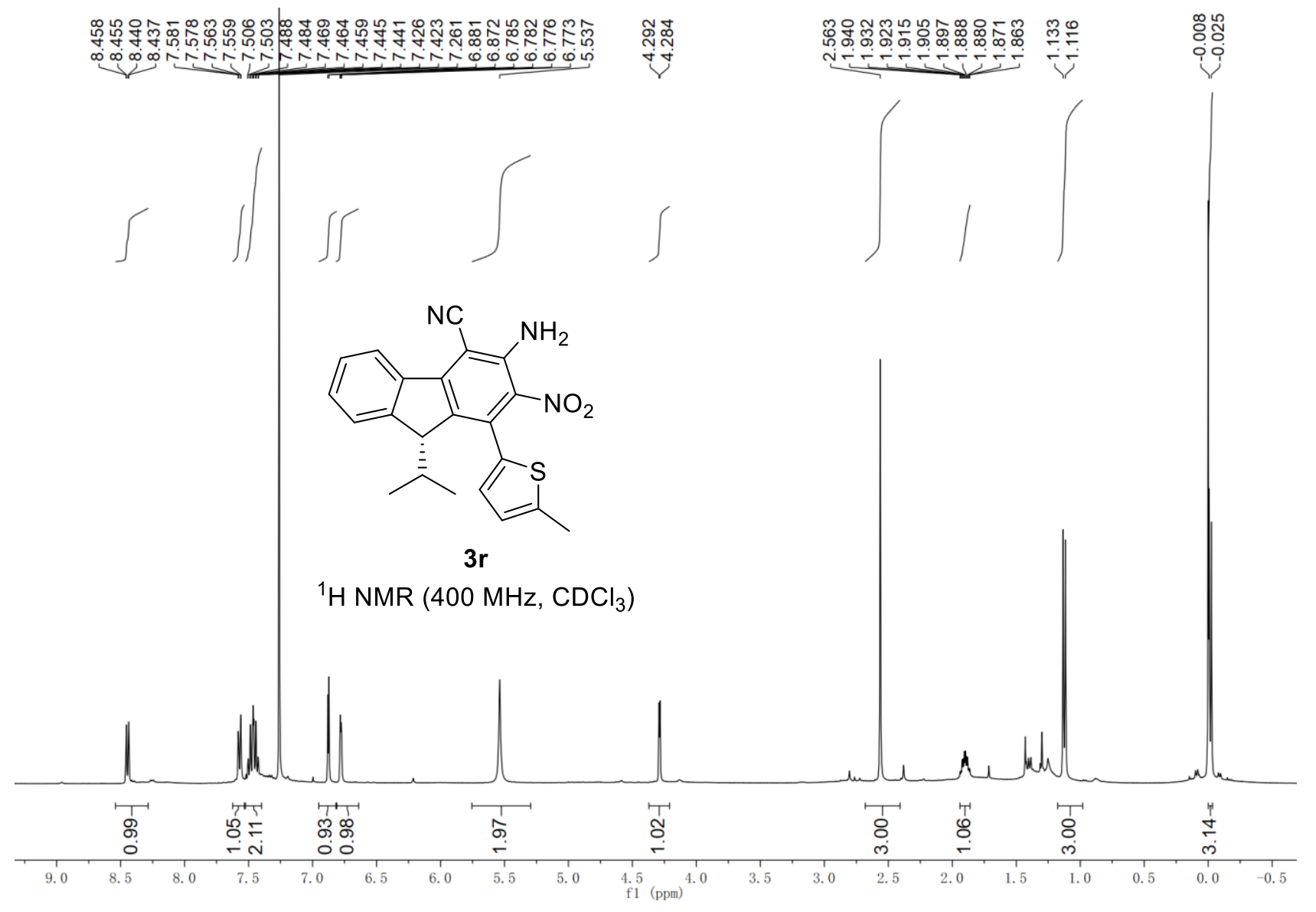

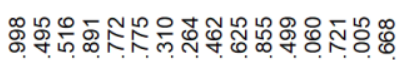

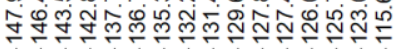

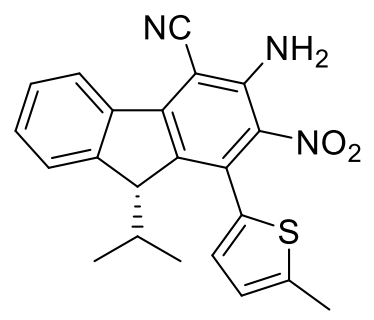

$3 r$

${ }^{13} \mathrm{C}$ NMR $\left(100 \mathrm{MHz}, \mathrm{CDCl}_{3}\right)$

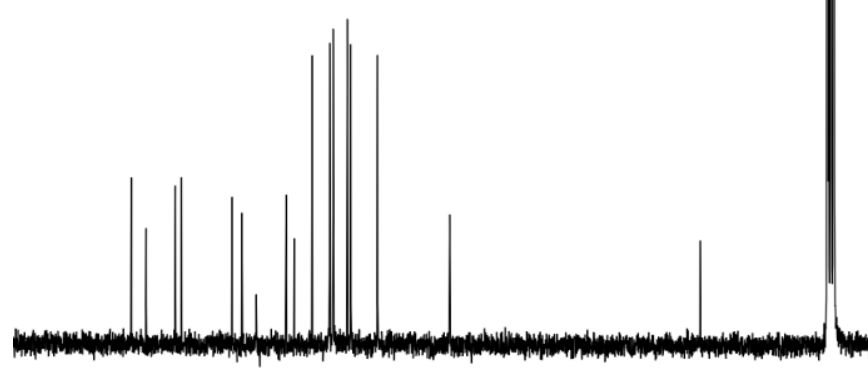

ָூ i

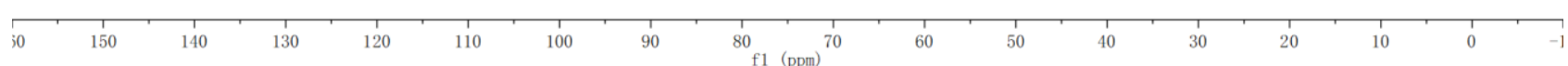



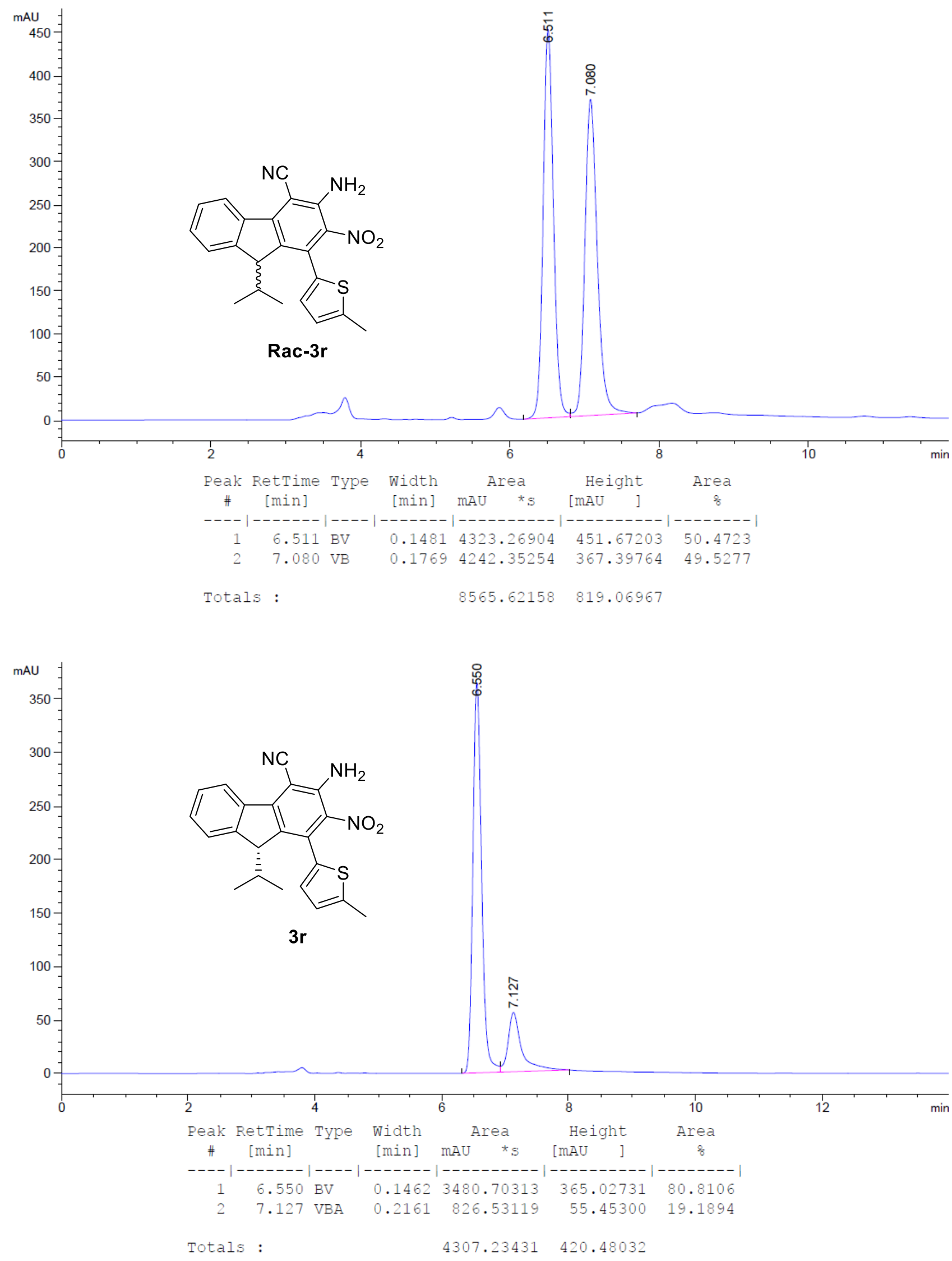


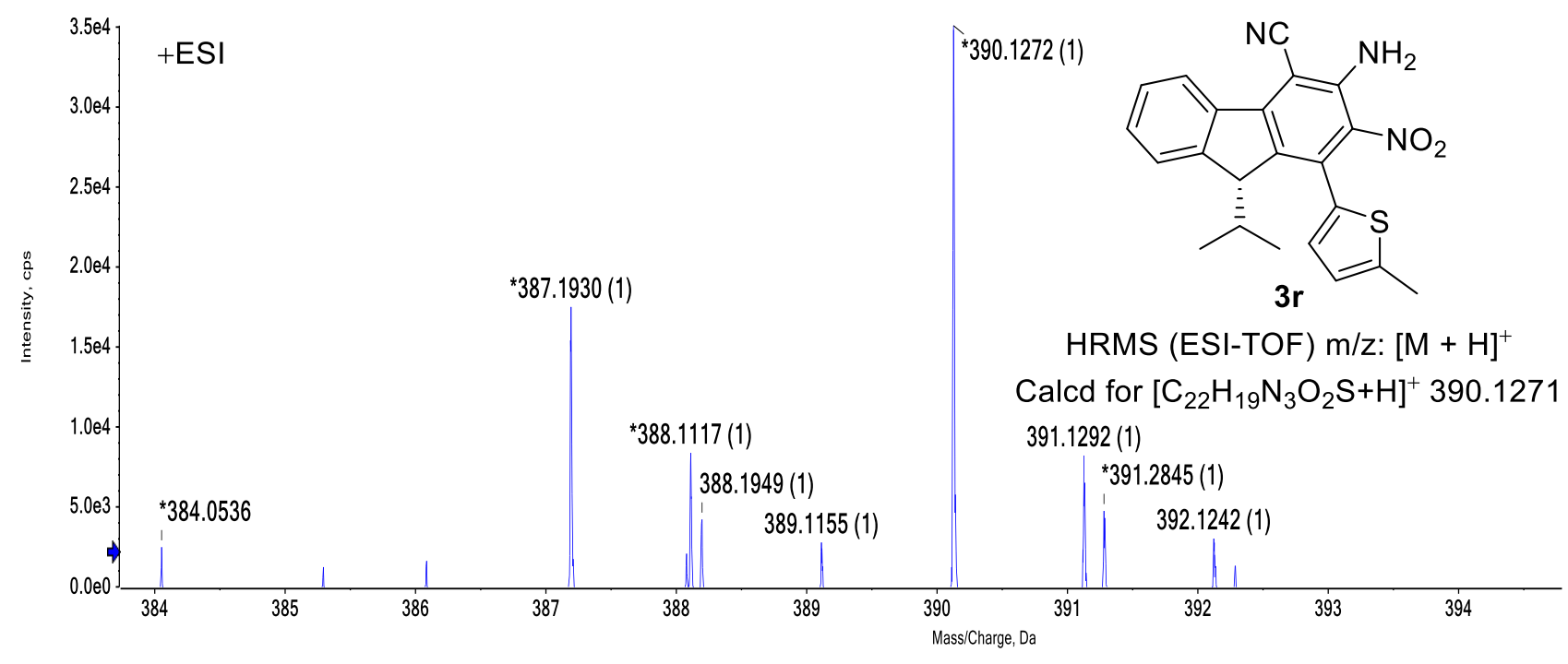



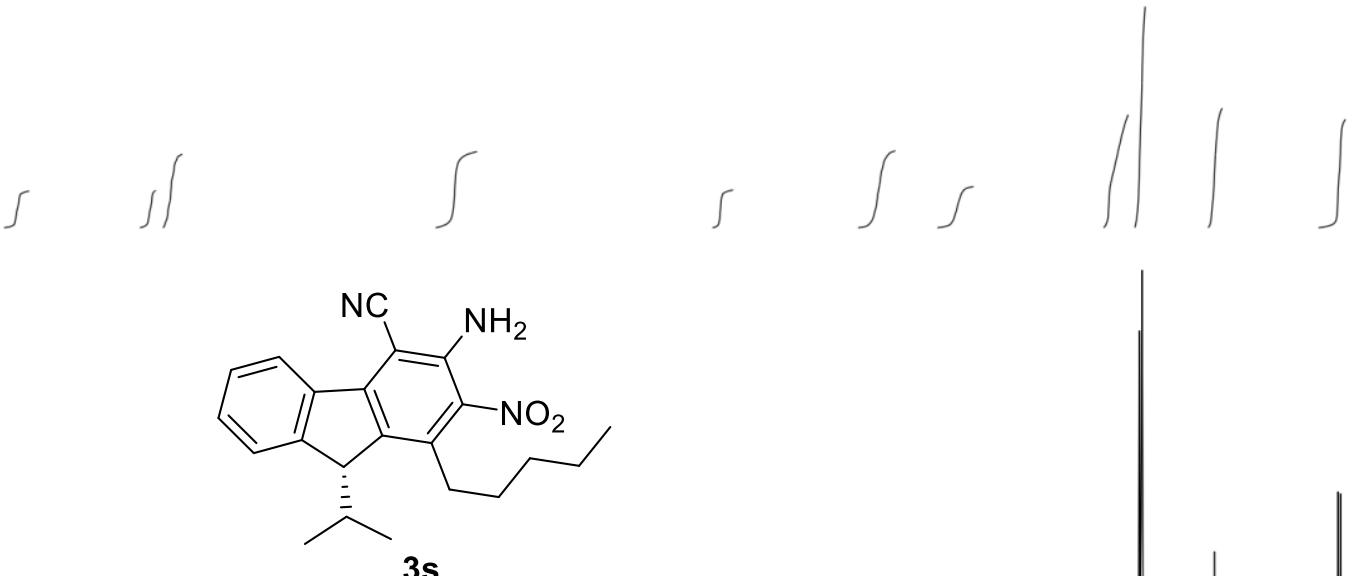

${ }^{1} \mathrm{H}$ NMR $\left(400 \mathrm{MHz}, \mathrm{CDCl}_{3}\right)$

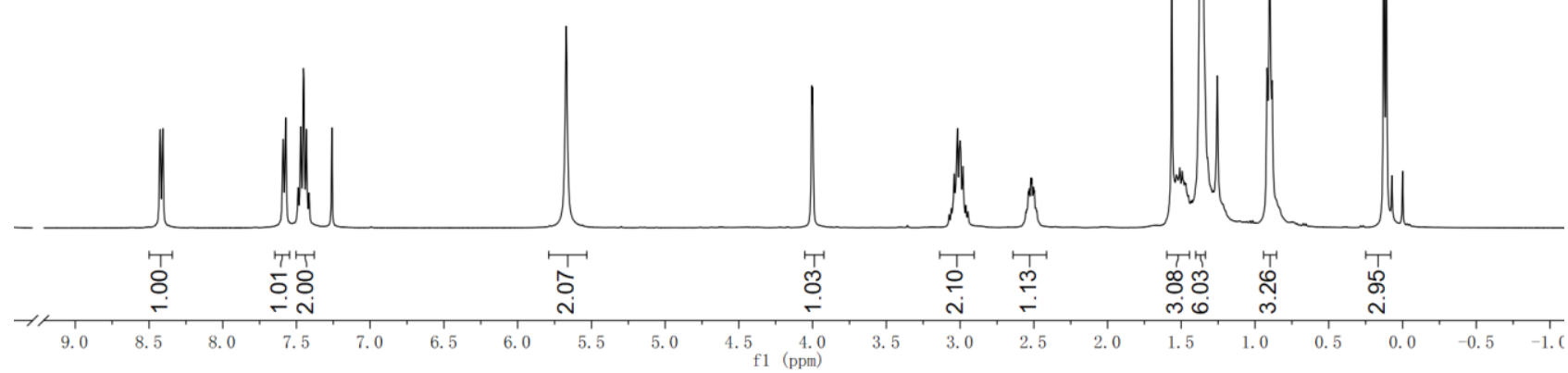

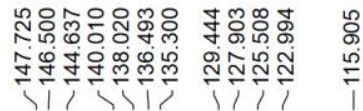

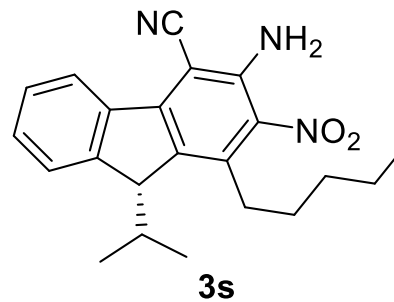

${ }^{13} \mathrm{C} \mathrm{NMR}\left(100 \mathrm{MHz}, \mathrm{CDCl}_{3}\right)$

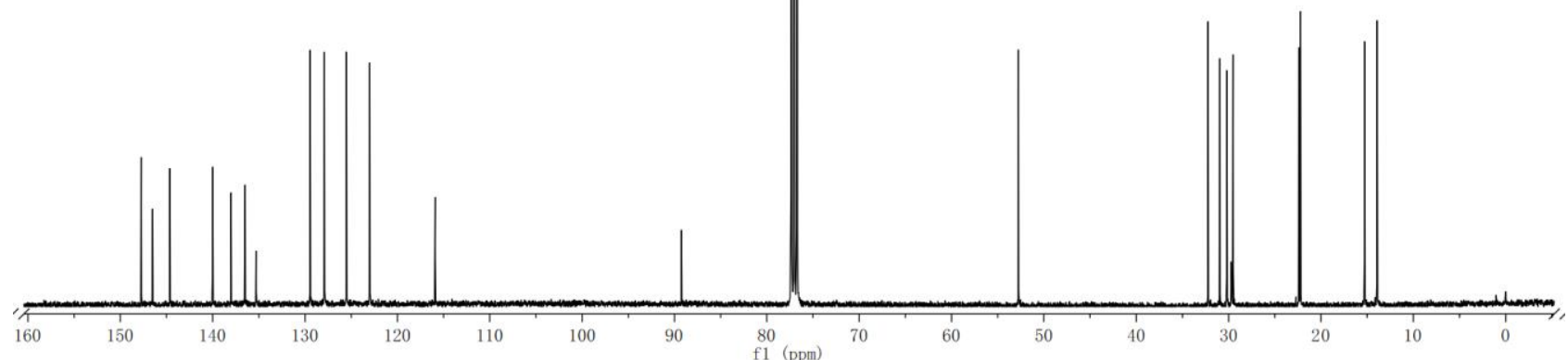




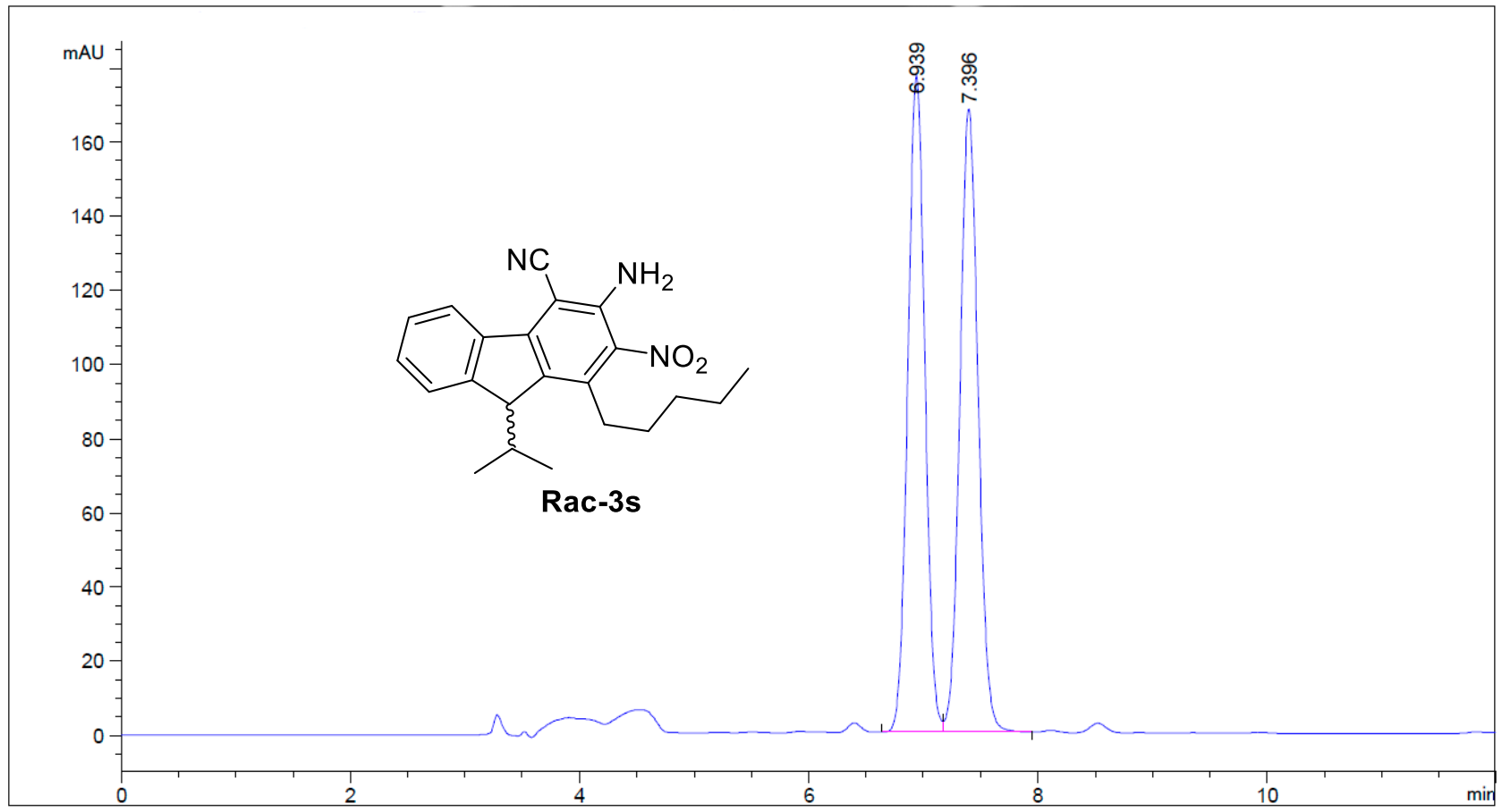

\begin{tabular}{cccccc} 
Peak No. & Ret Time & Width & Height & Area & Area [\%] \\
\hline 1 & 6.939 & 0.1617 & 1838.98669 & 177.37163 & 49.7062 \\
2 & 7.396 & 0.1714 & 1860.72888 & 168.07829 & 50.2938
\end{tabular}

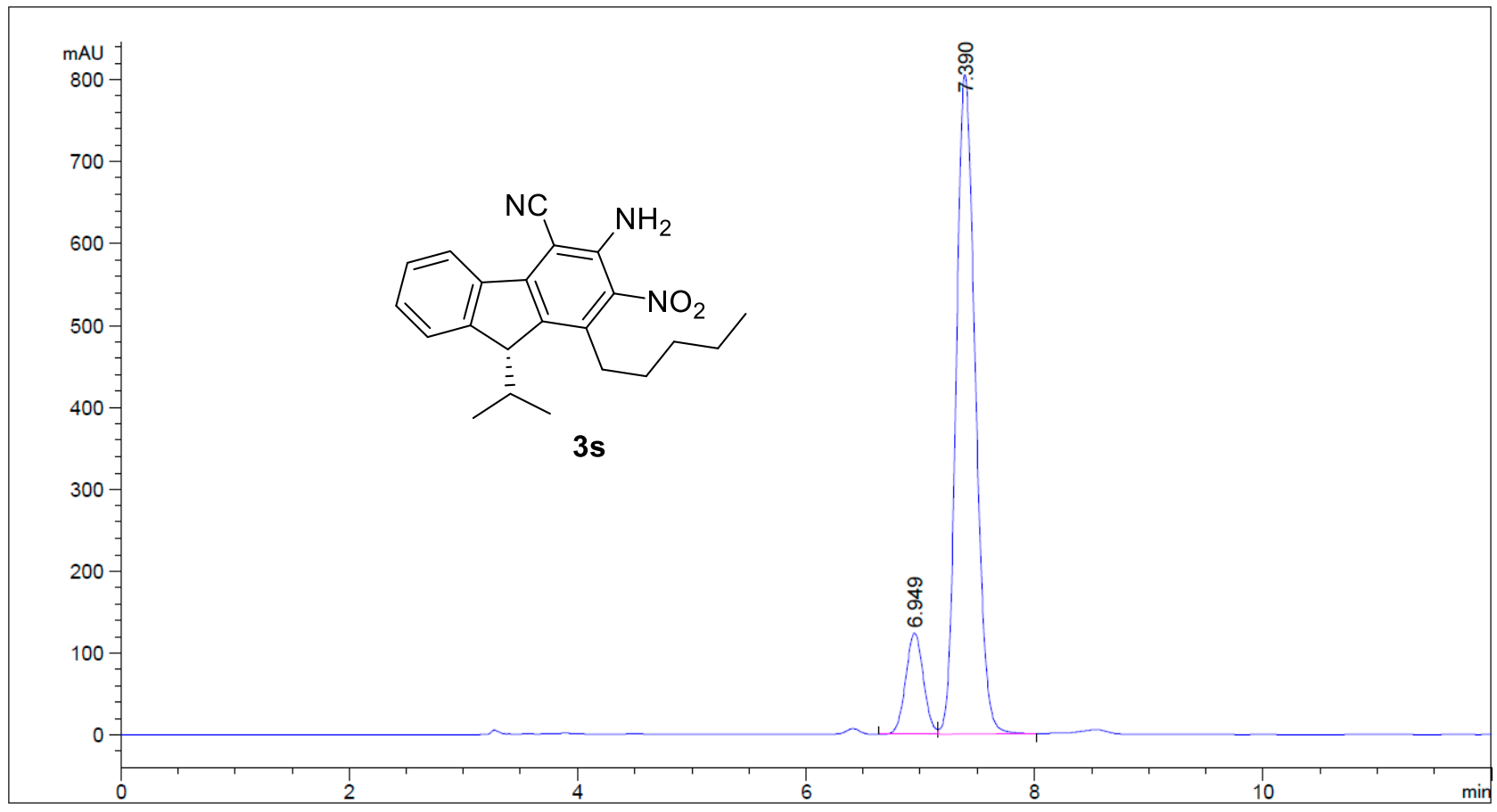

\begin{tabular}{clcccc} 
Peak No. & Ret Time & Width & Height & Area & Area [\%] \\
\hline 1 & 6.949 & 0.1680 & 1331.17114 & 123.52210 & 12.4725 \\
2 & 7.390 & 0.1820 & 9341.66016 & 804.98499 & 87.5275
\end{tabular}




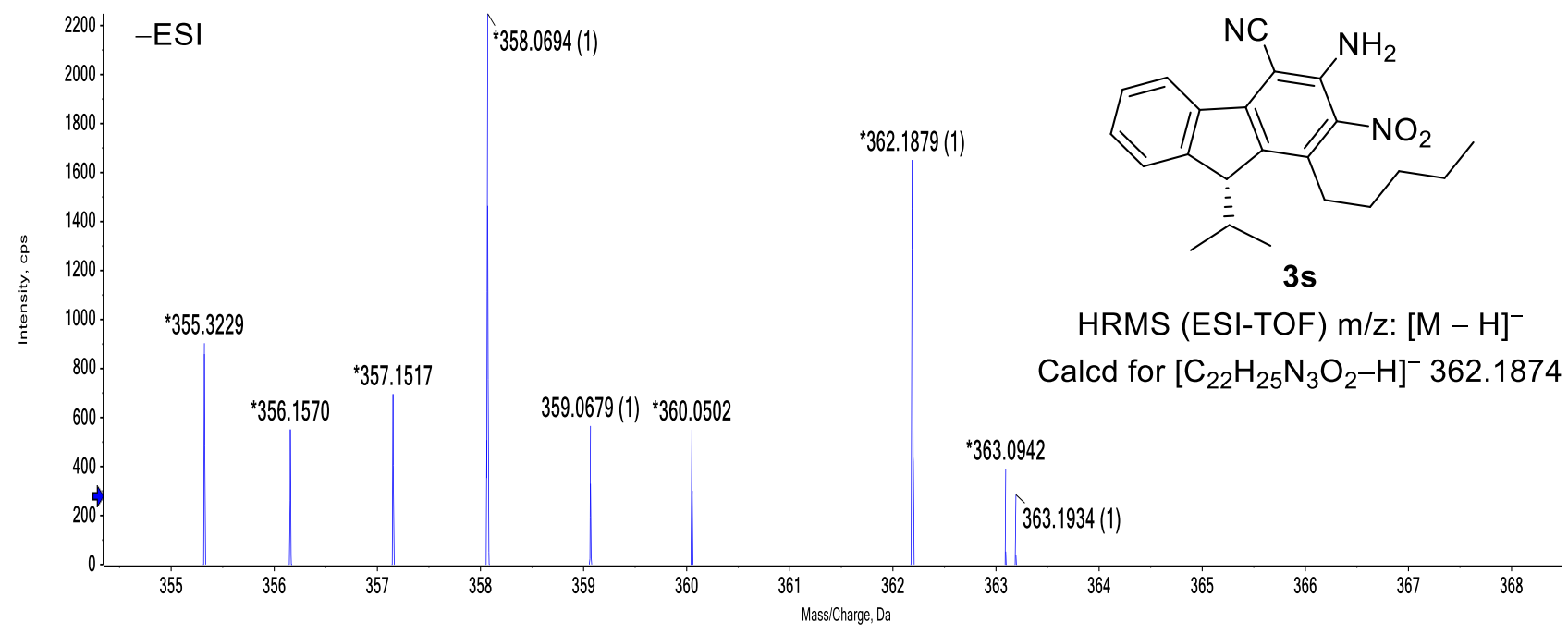



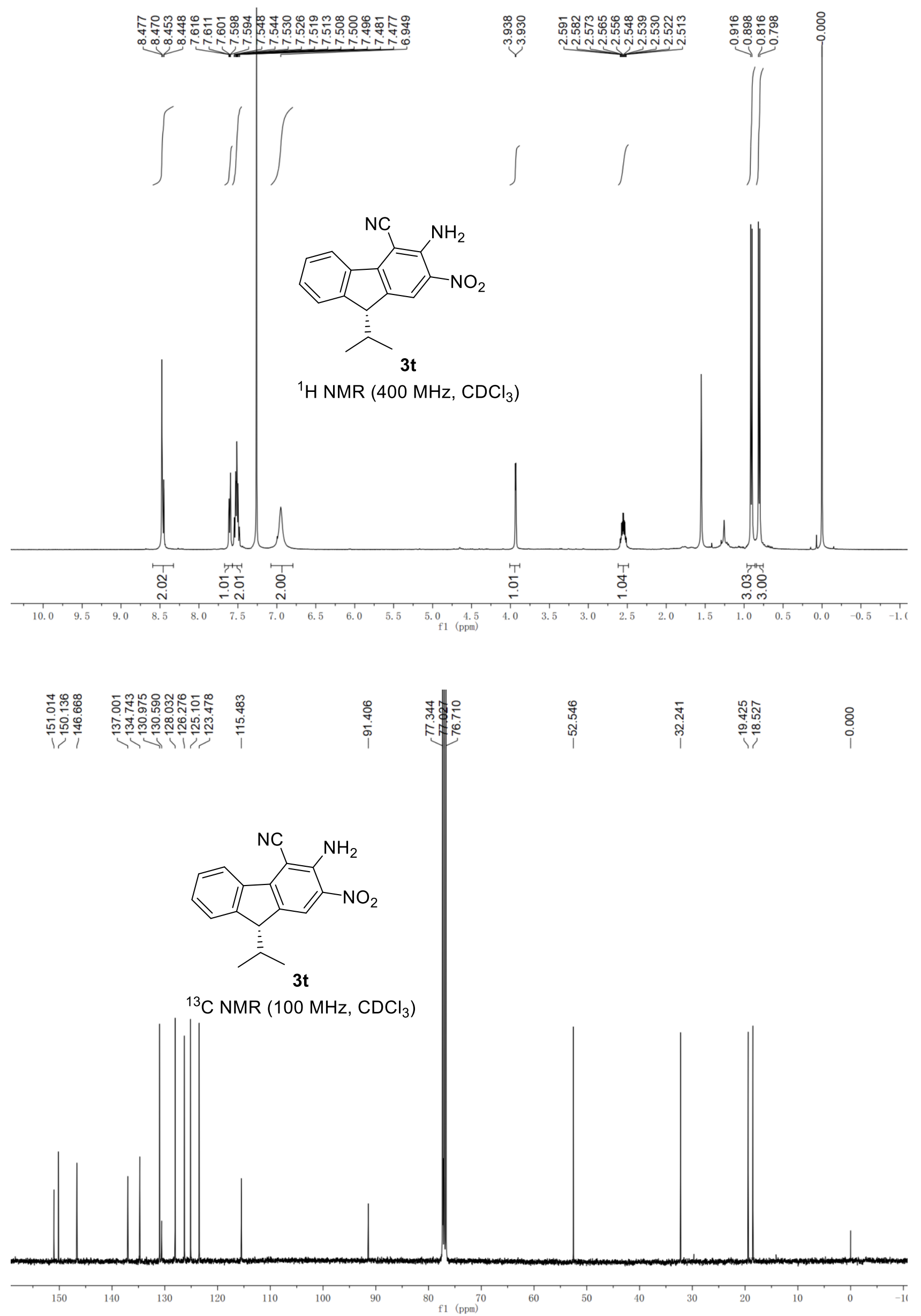


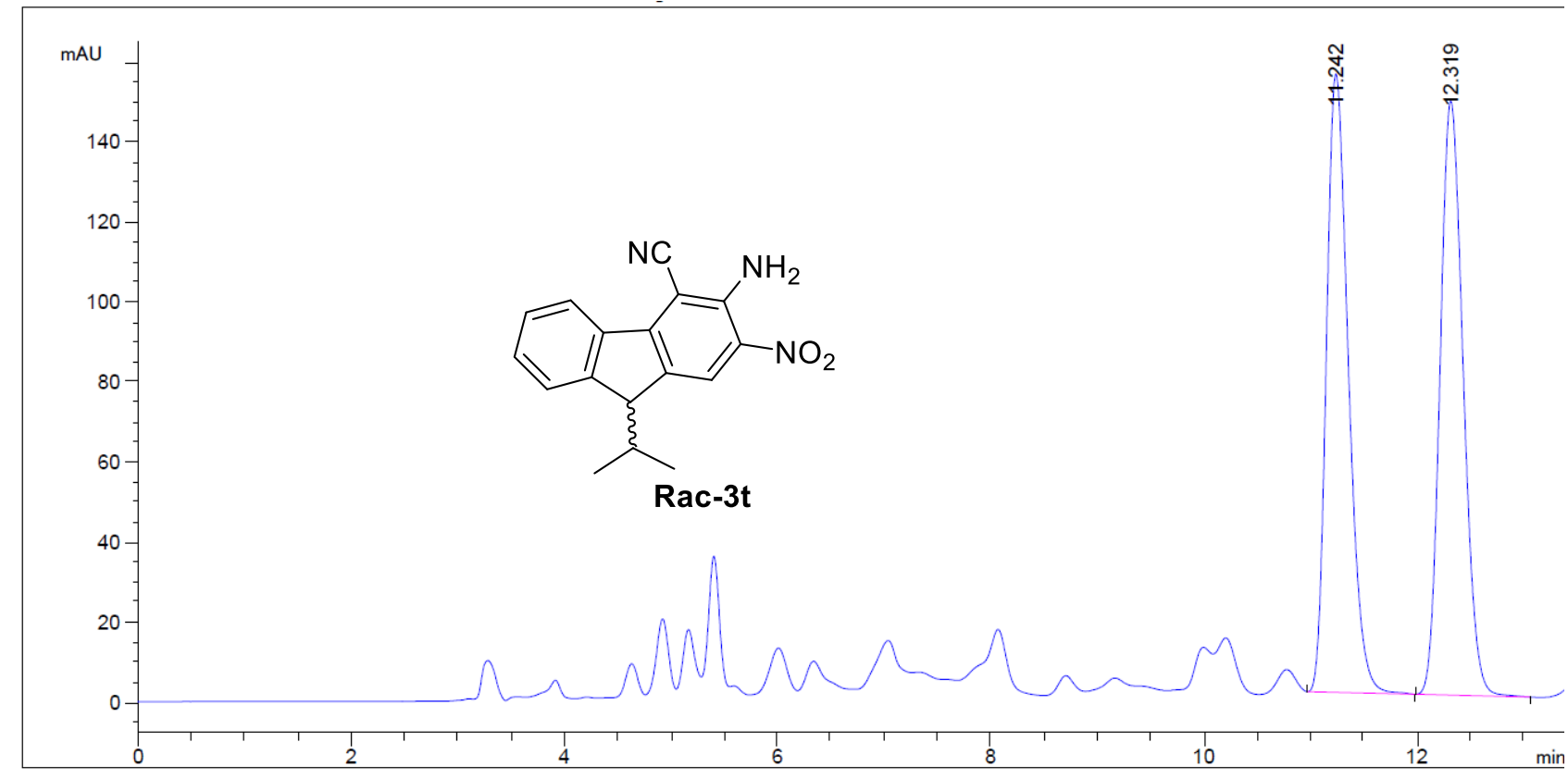

\begin{tabular}{|c|c|c|c|c|c|c|}
\hline Peak & RetTime & Type & Width & Area & Height & Area \\
\hline \# & [min] & & [min] & $\mathrm{mAU} \quad * \mathrm{~s}$ & {$[\mathrm{mAU} \quad]$} & $8_{8}$ \\
\hline & & & & 984 & & \\
\hline , & 2.31 & $B A$ & 351 & 2234.05859 & .56342 & 3729 \\
\hline
\end{tabular}

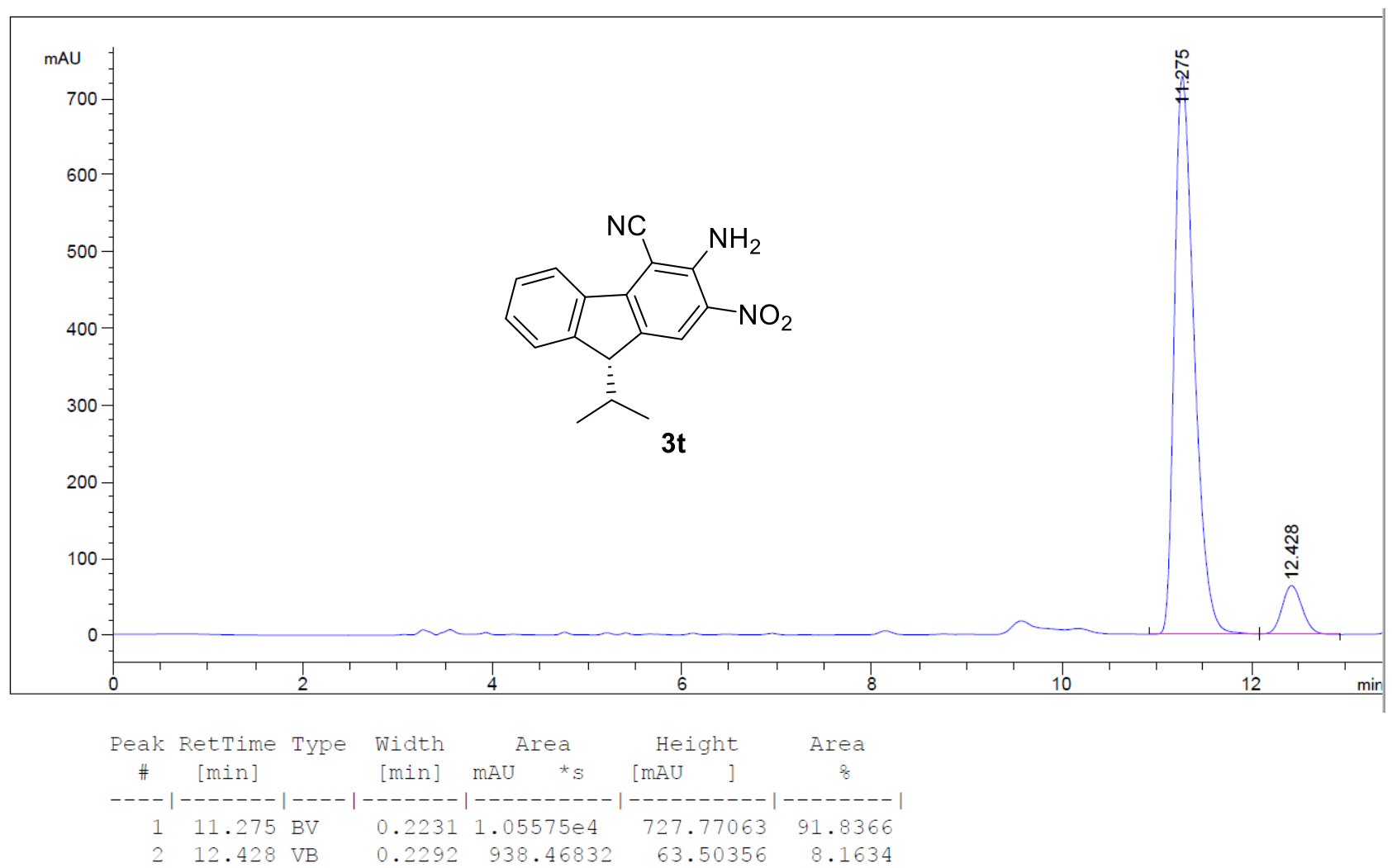




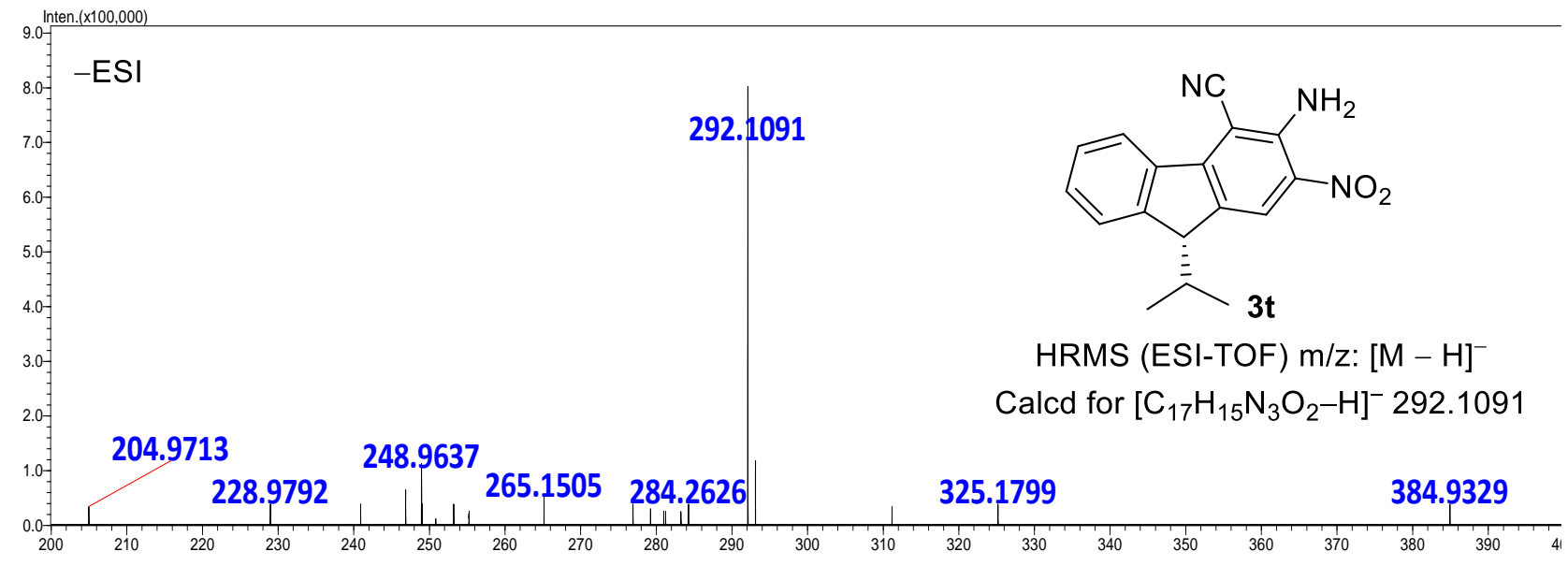


<smiles>CC(C)[C@H]1c2ccccc2-c2c(C#N)c(N)c([N+](=O)[O-])c(C3CCCCC3)c21</smiles>

$3 \mathbf{t}^{\prime}$

${ }^{1} \mathrm{H}$ NMR $\left(600 \mathrm{MHz}, \mathrm{CDCl}_{3}\right)$

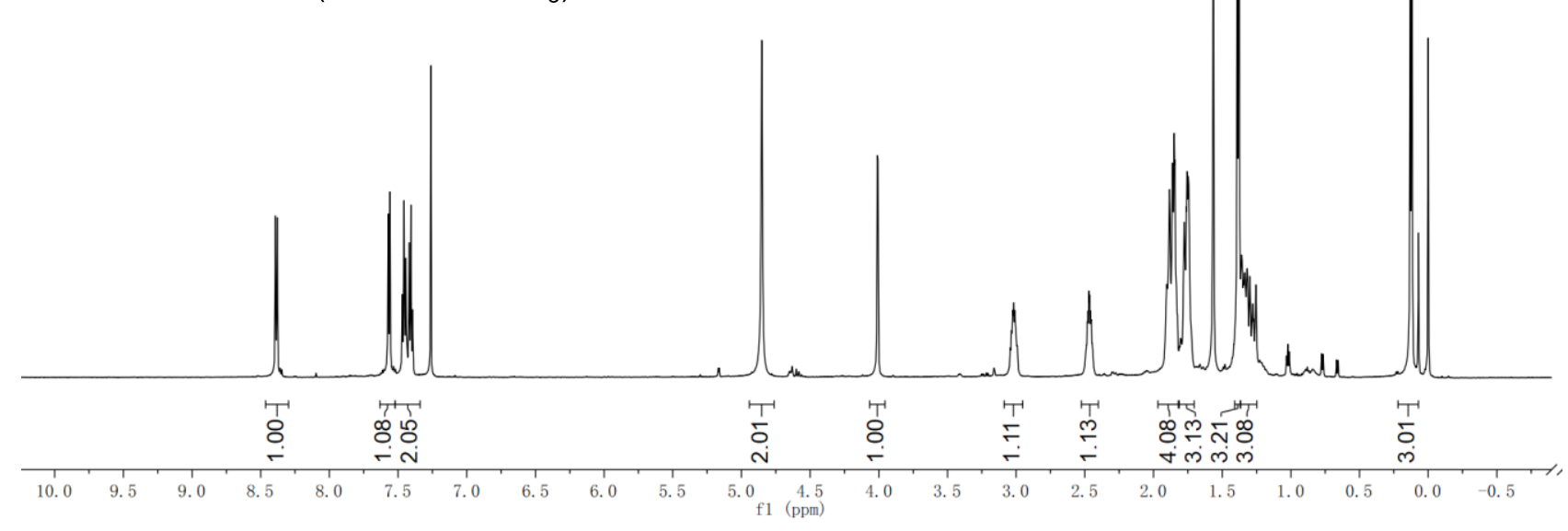<smiles>CC(C)[C@H]1c2ccccc2-c2c(C#N)c(N)c([N+](=O)[O-])c(C3CCCCC3)c21</smiles>

${ }^{13} \mathrm{C}$ NMR $\left(150 \mathrm{MHz}, \mathrm{CDCl}_{3}\right)$

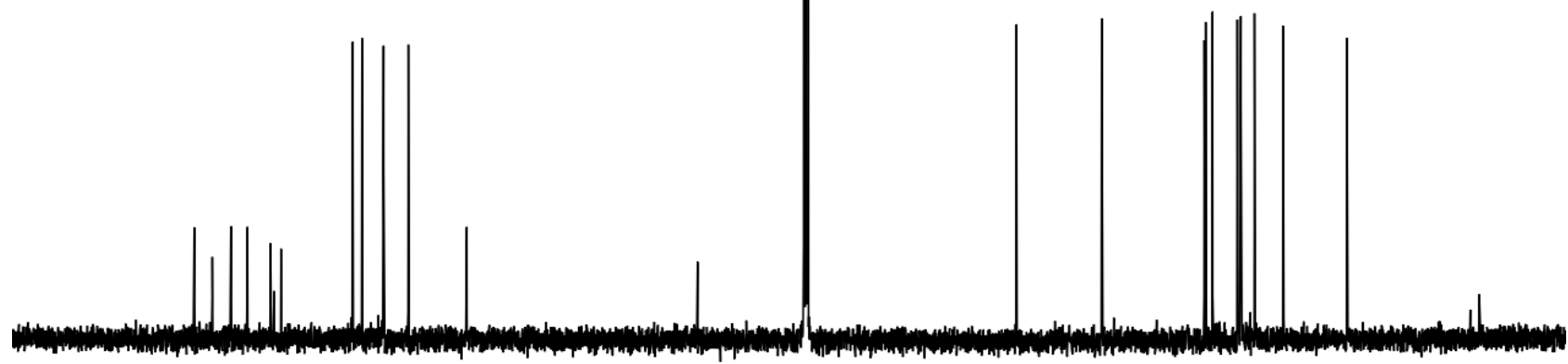




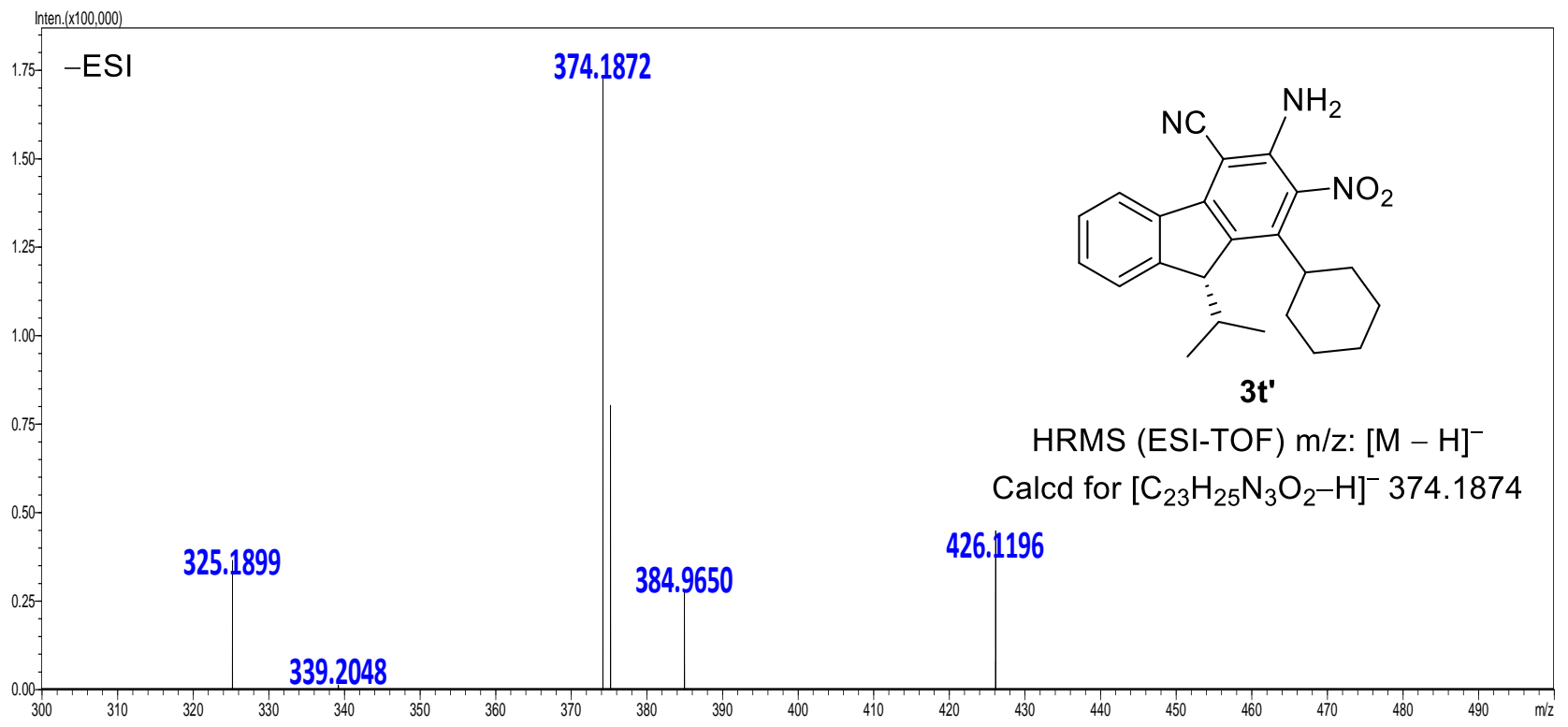



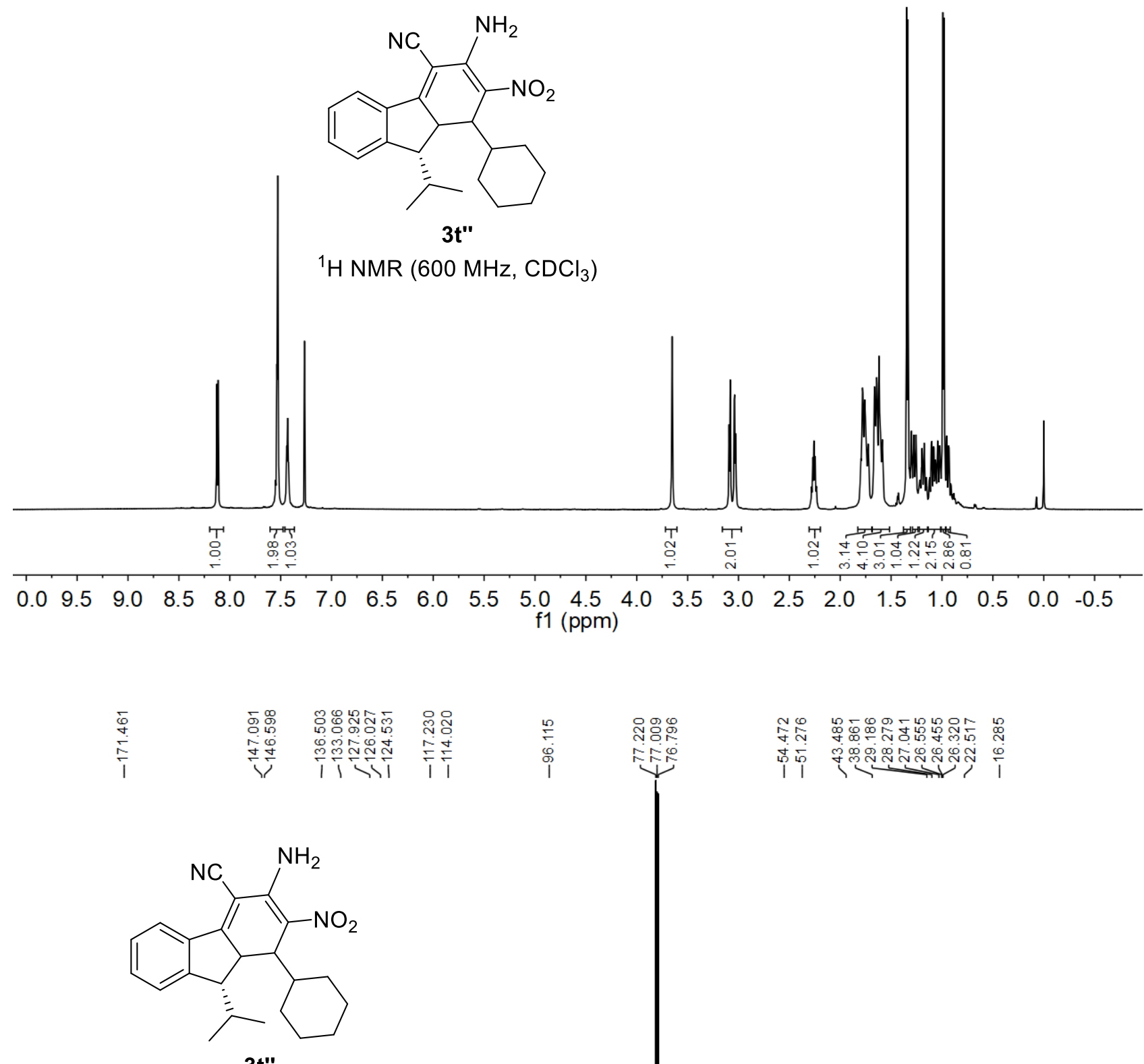

3t"

${ }^{13} \mathrm{C} \mathrm{NMR}\left(150 \mathrm{MHz}, \mathrm{CDCl}_{3}\right)$

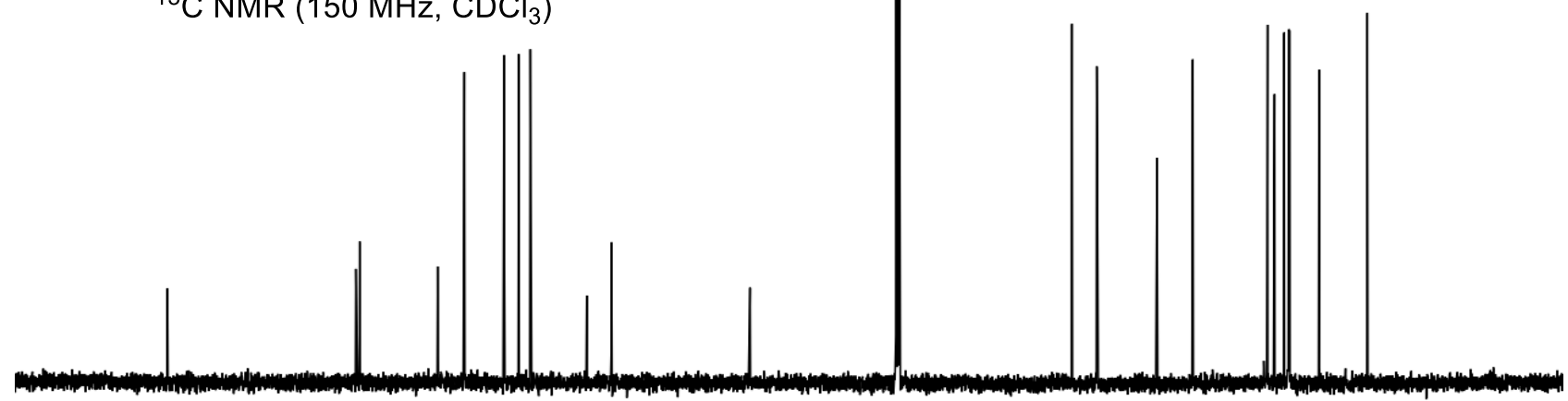

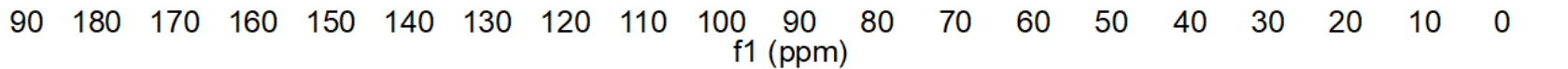




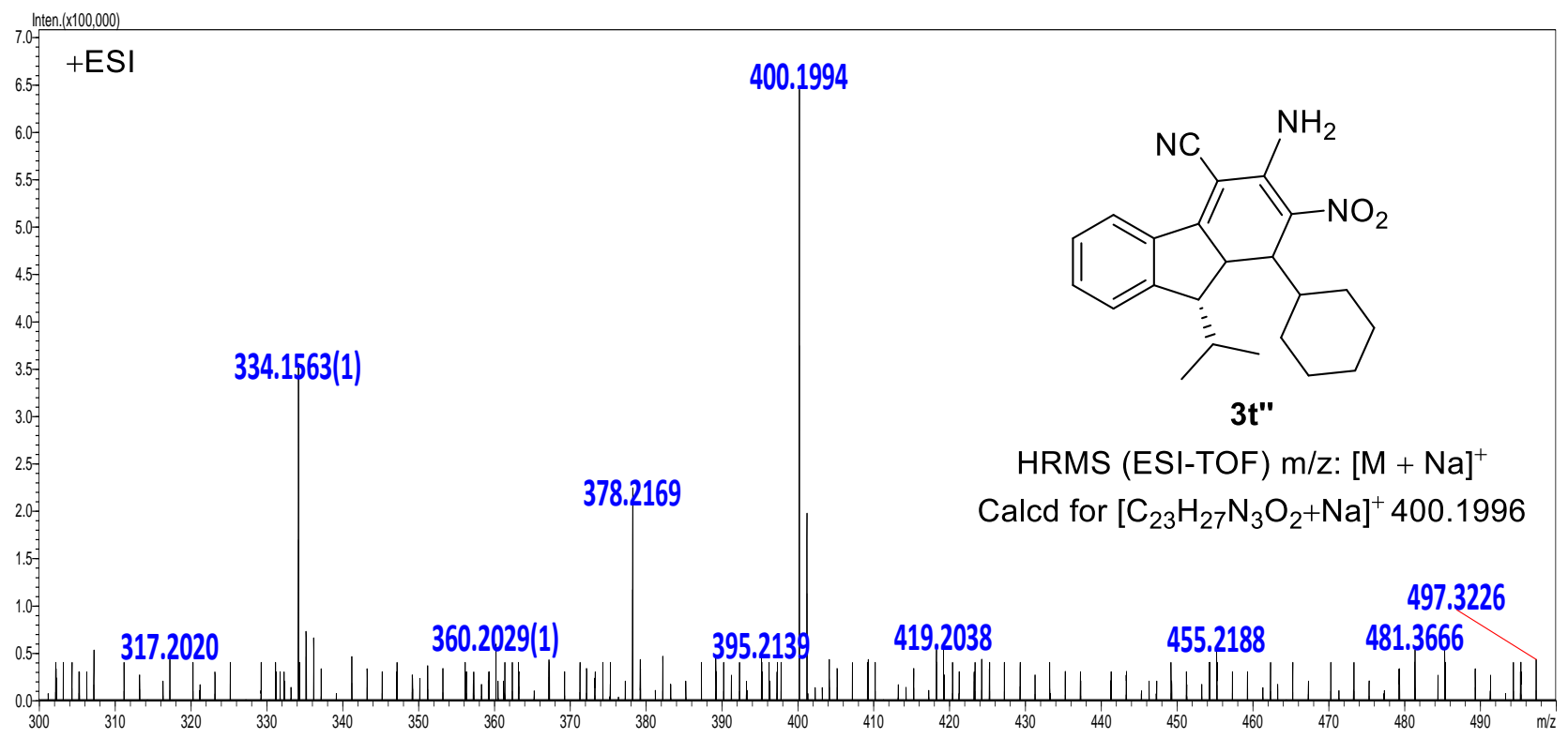




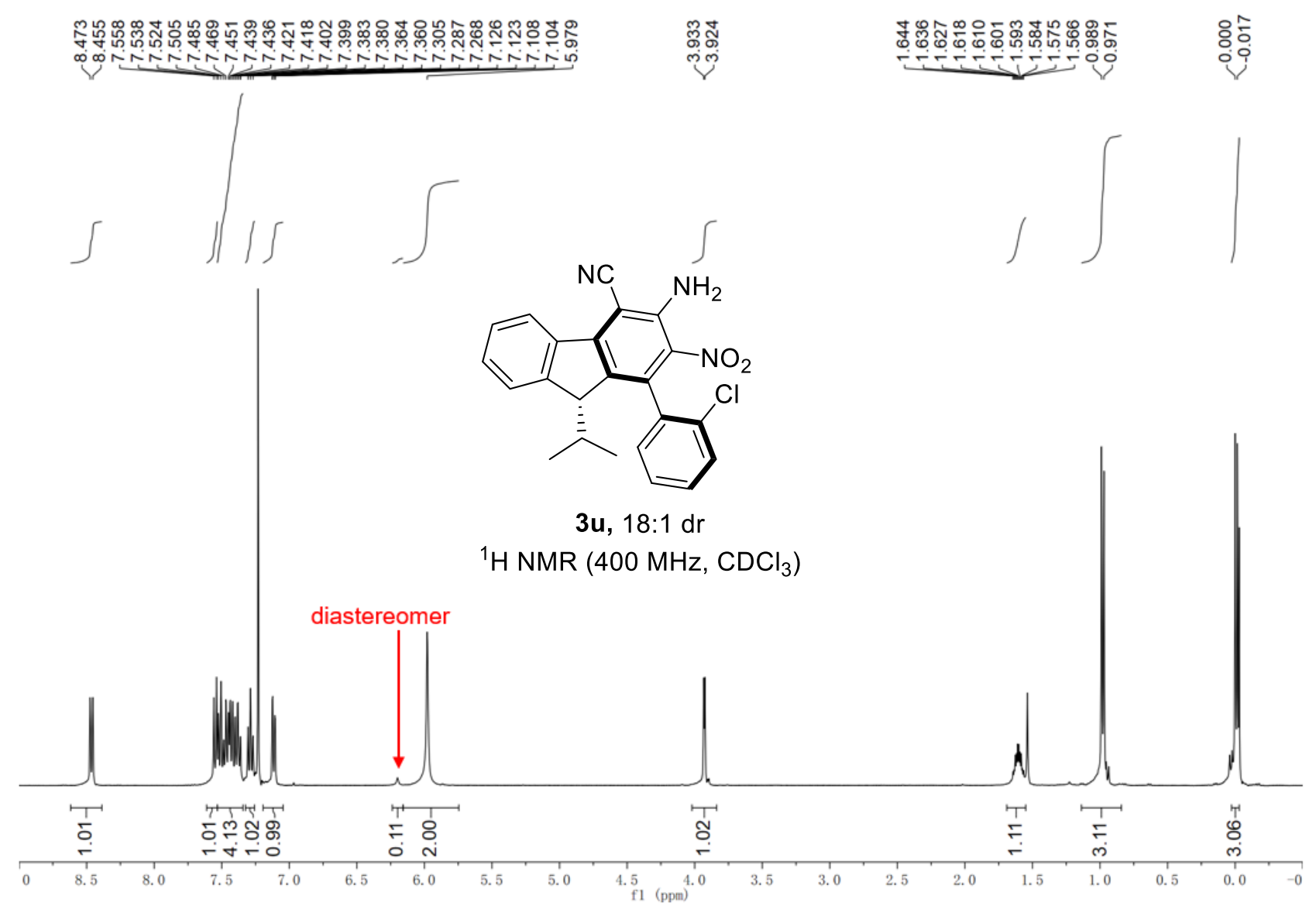

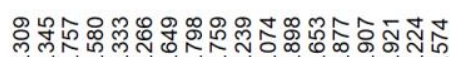

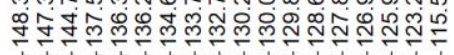

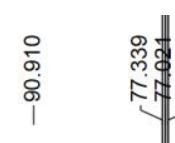

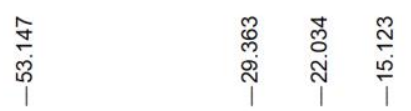

웅

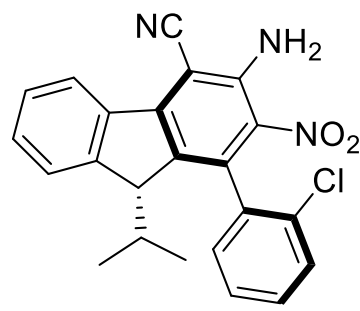

3u, $18: 1 \mathrm{dr}$

${ }^{13} \mathrm{C}$ NMR $\left(100 \mathrm{MHz}, \mathrm{CDCl}_{3}\right)$

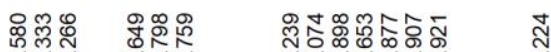

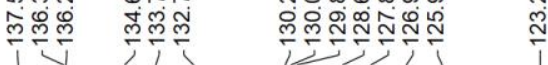
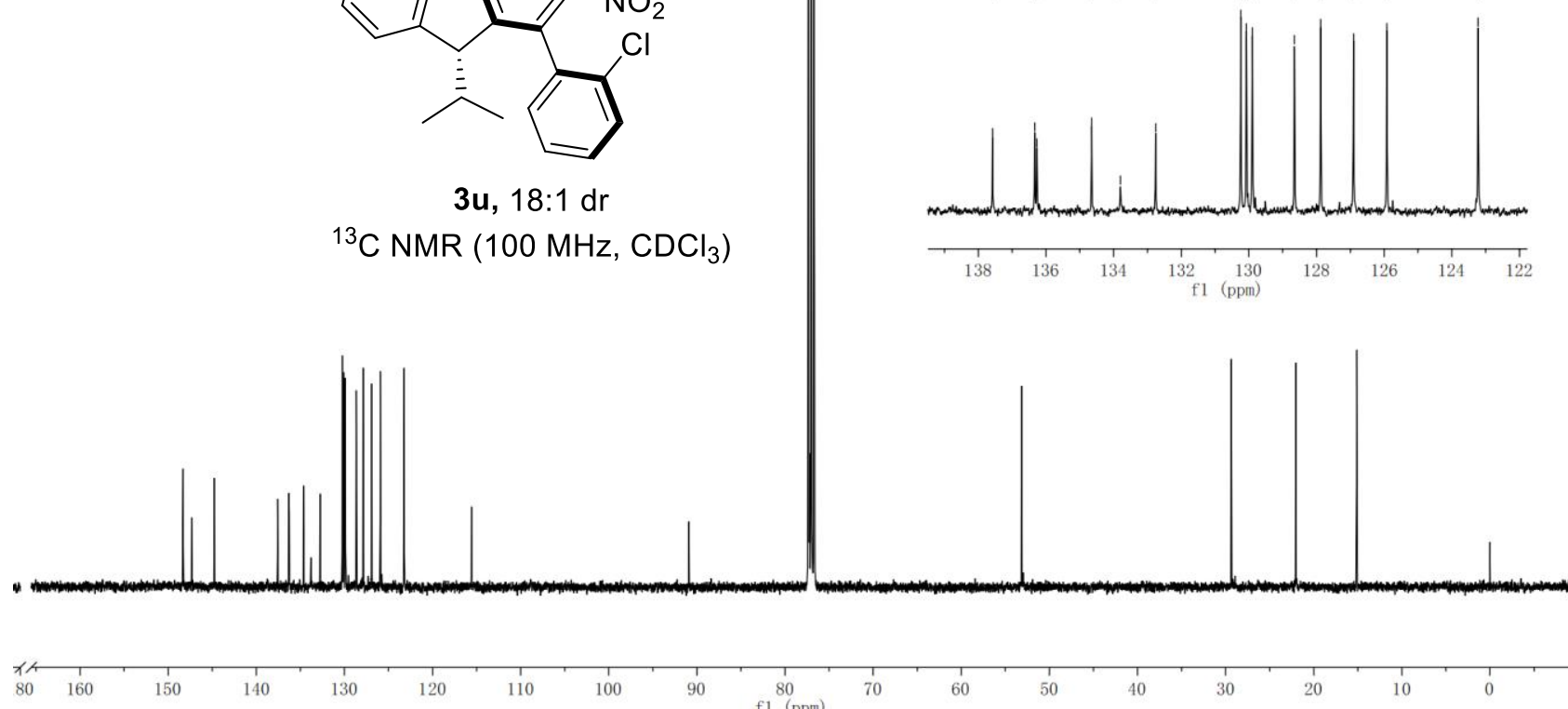

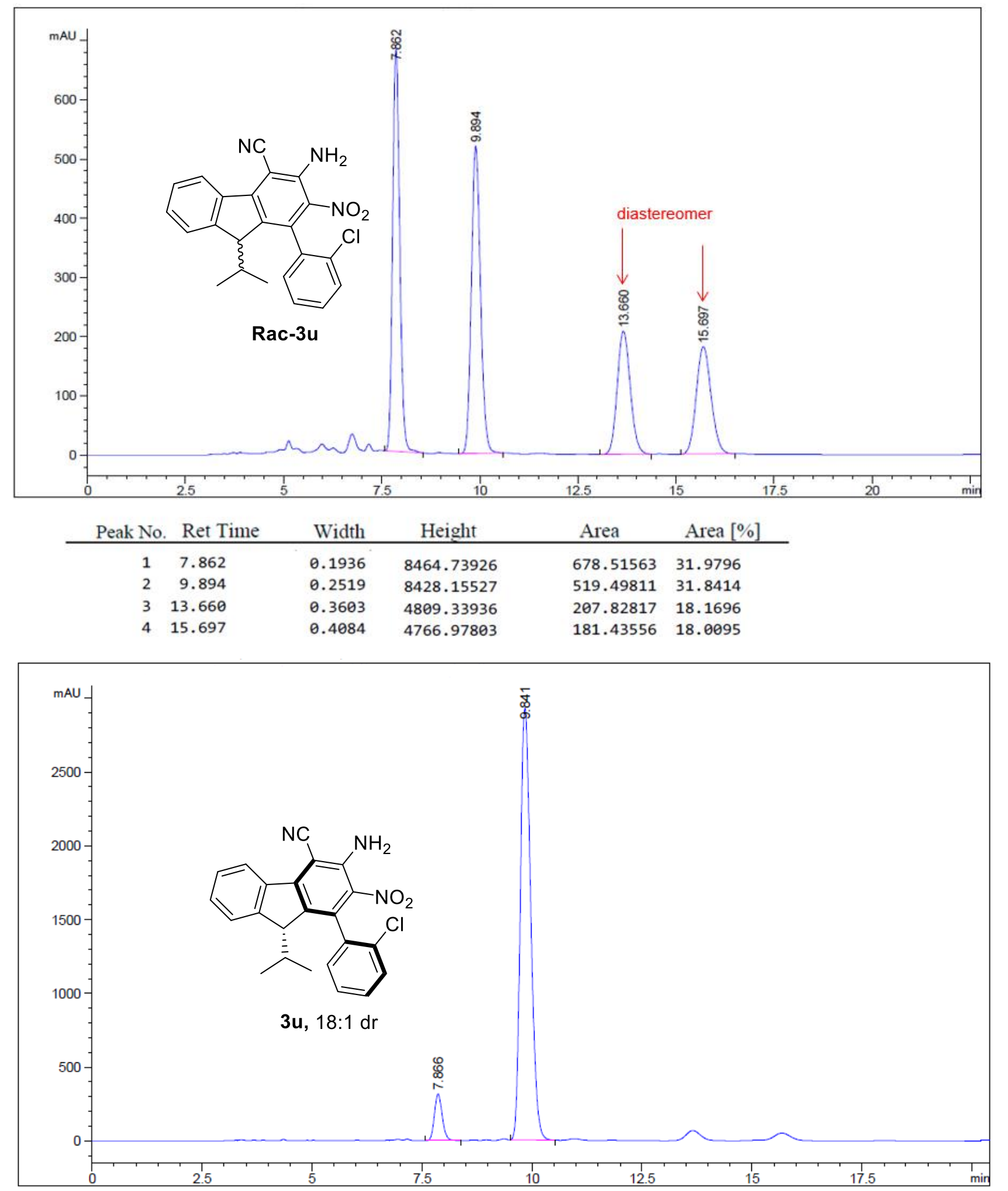

\begin{tabular}{clllrr} 
Peak No. & Ret Time & Width & Height & \multicolumn{1}{c}{ Area } & Area [\%] \\
\hline 1 & 7.866 & 0.1901 & 3900.08813 & 317.16605 & 7.5121 \\
2 & 9.841 & 0.2553 & $4.80177 \mathrm{e} 4$ & 2929.62207 & 92.4879
\end{tabular}




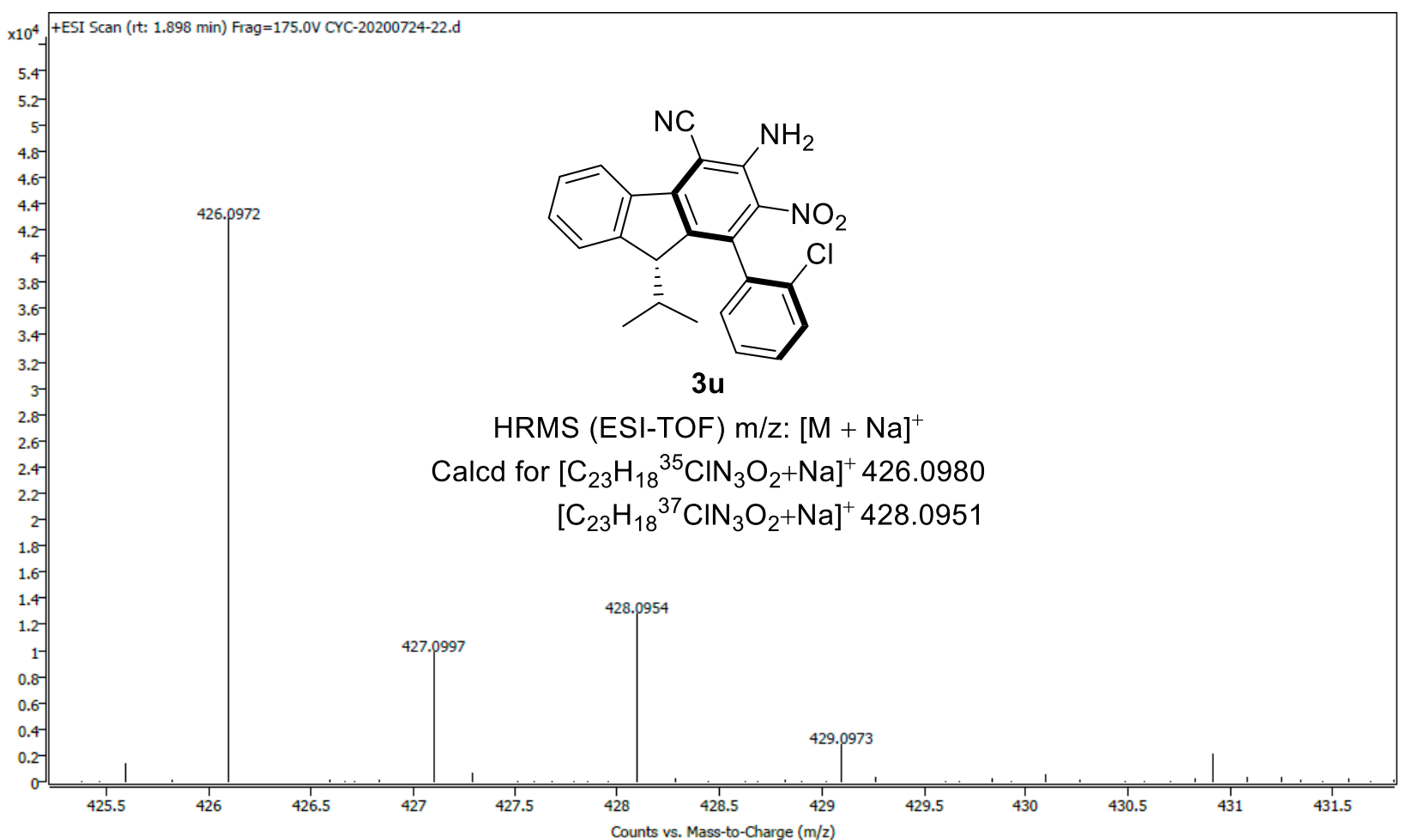



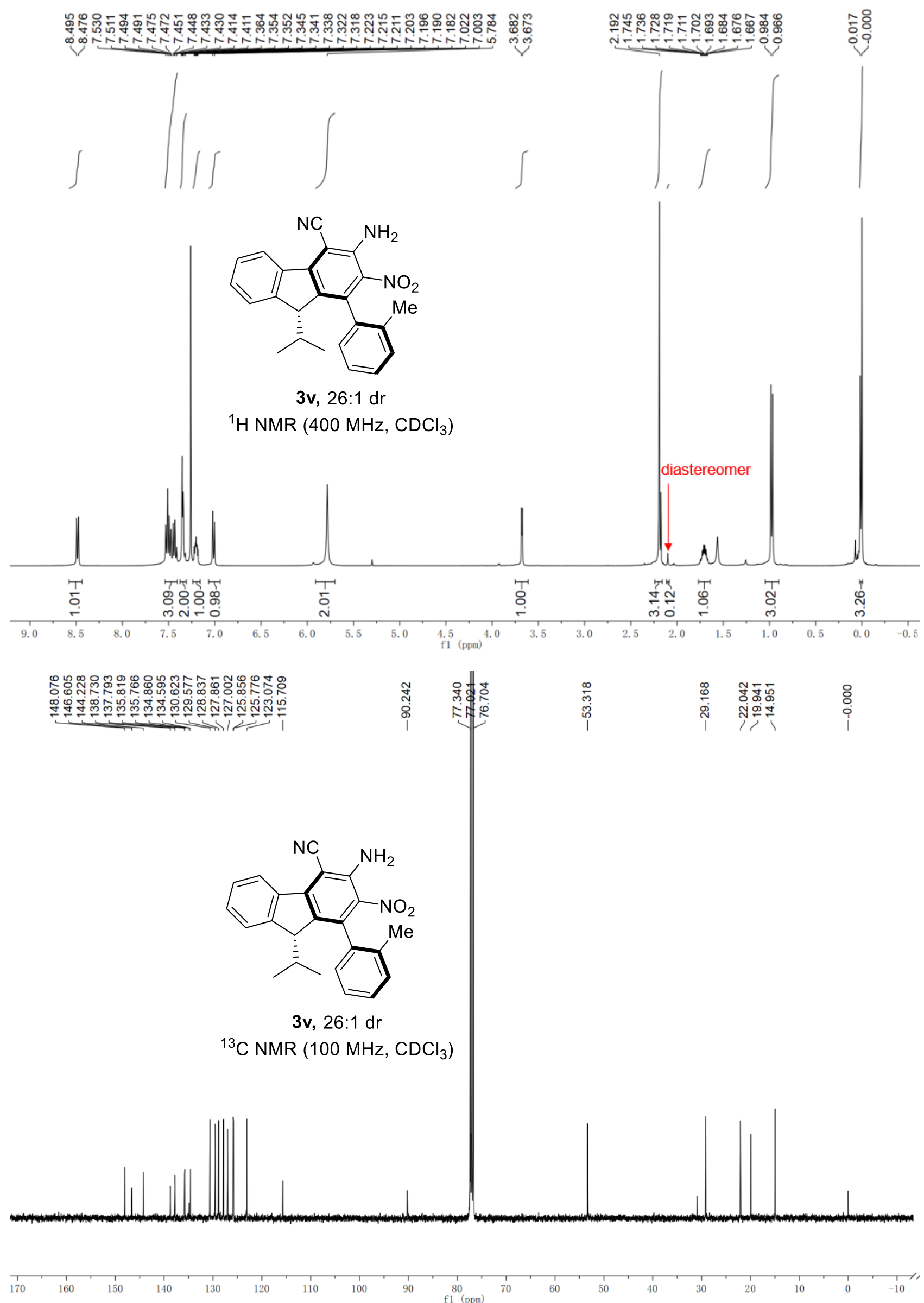

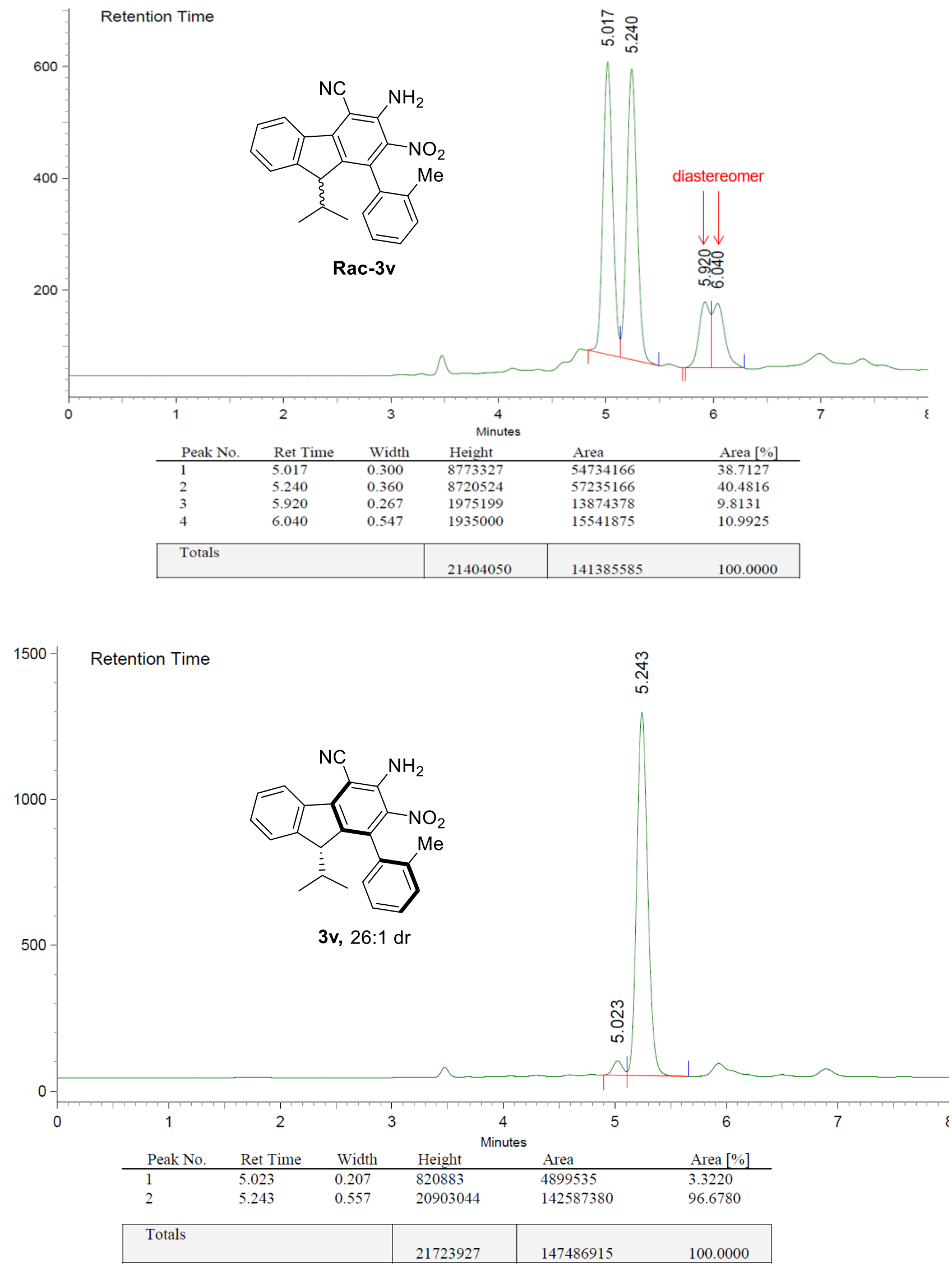


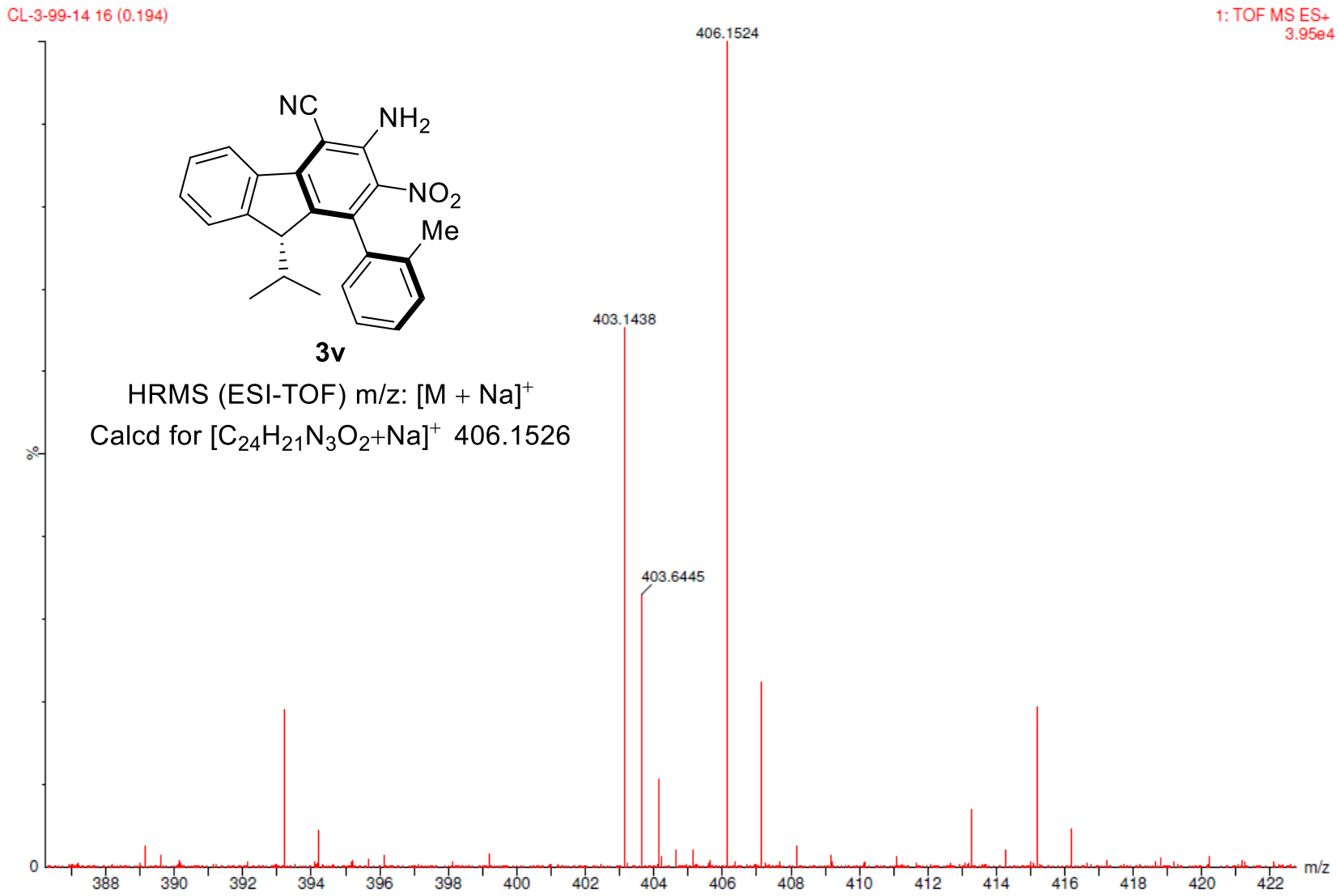



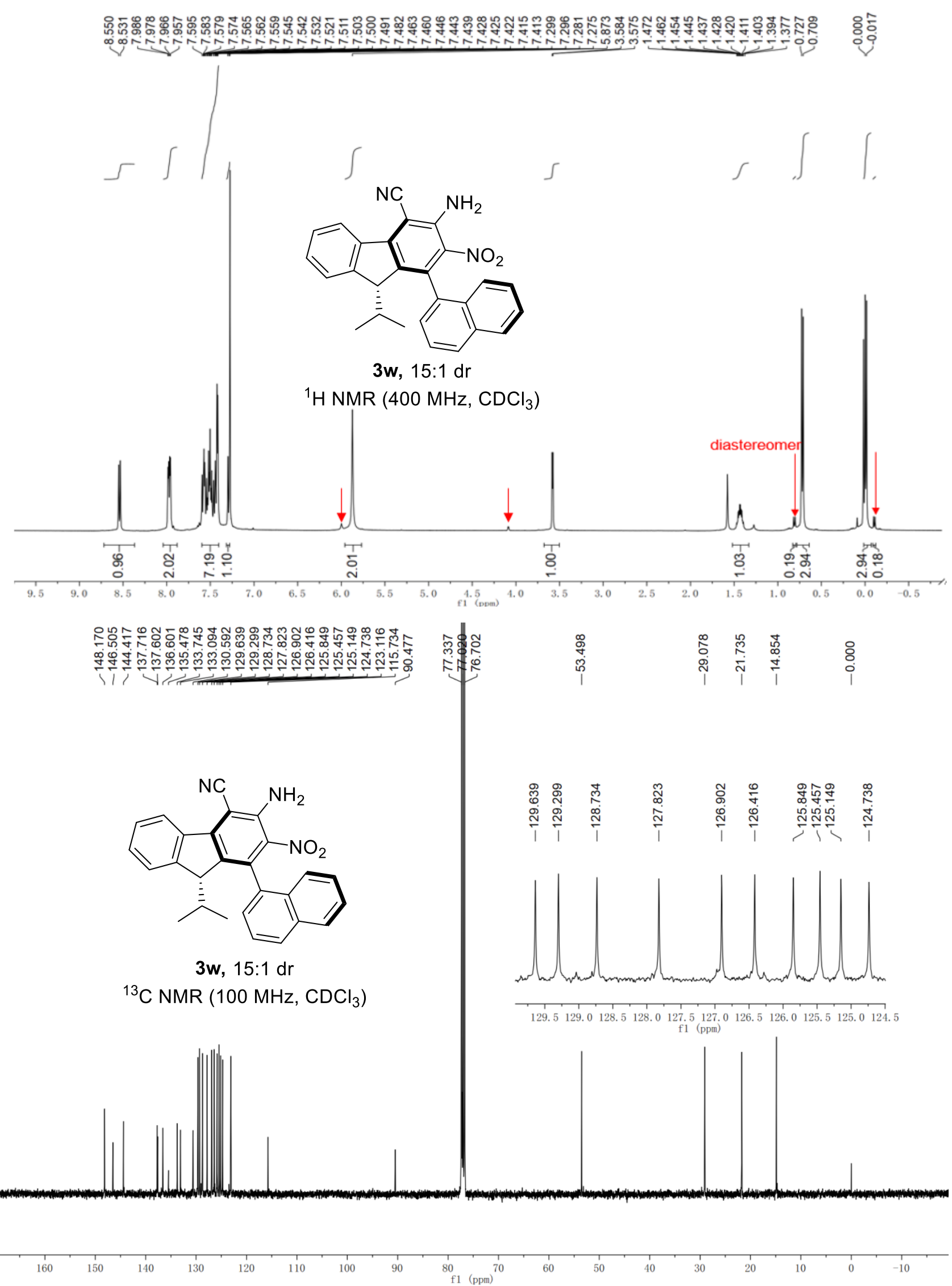

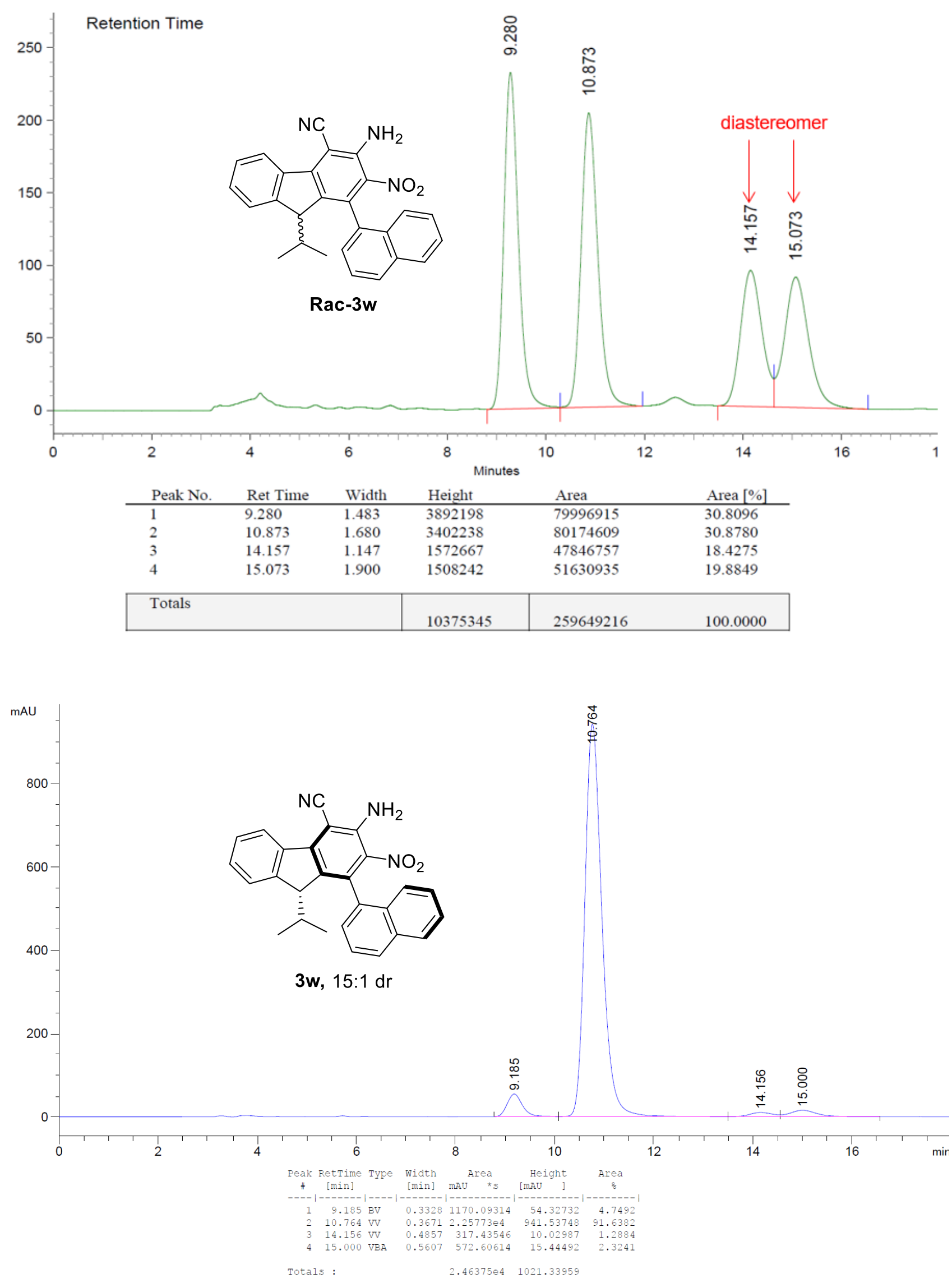


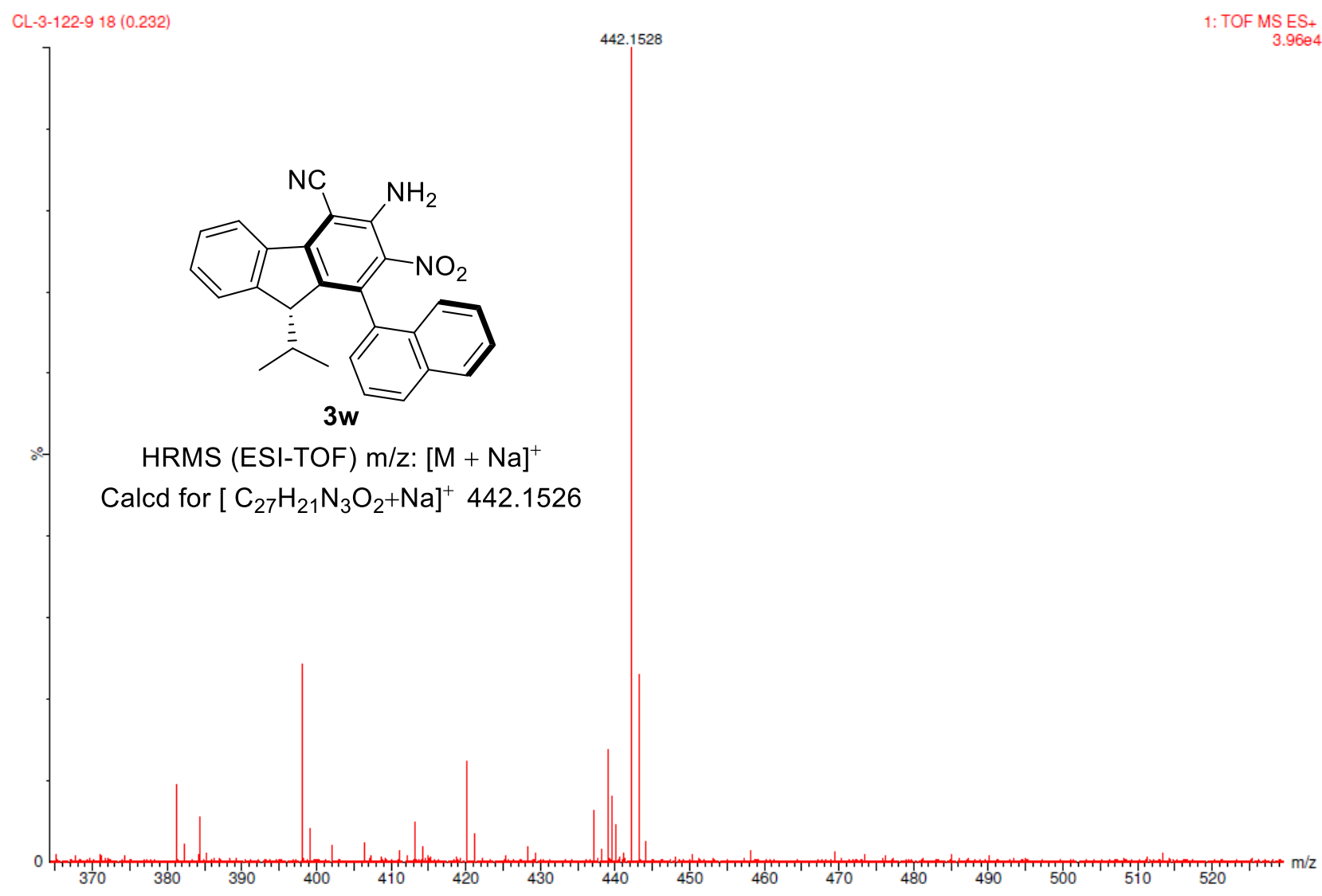



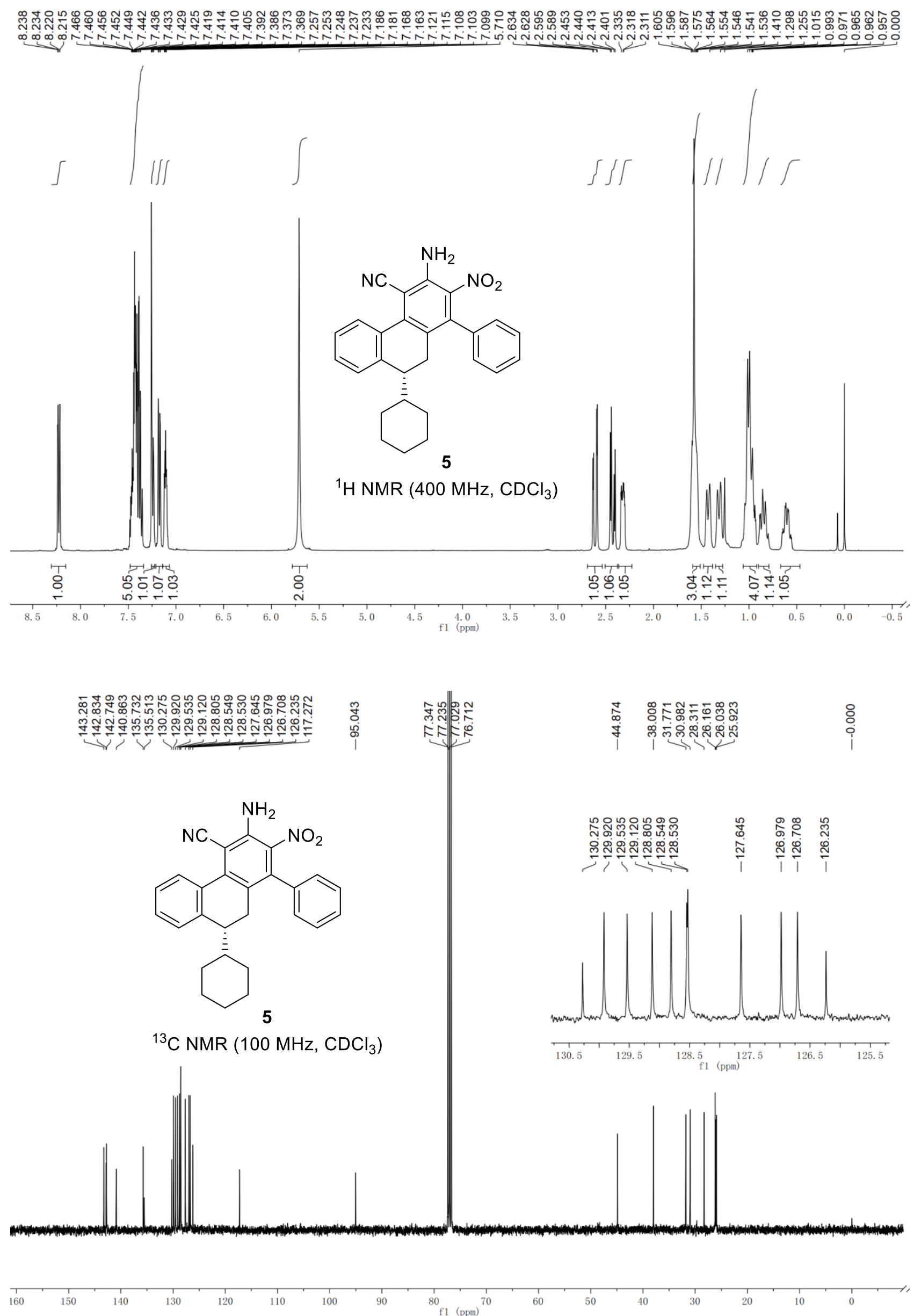


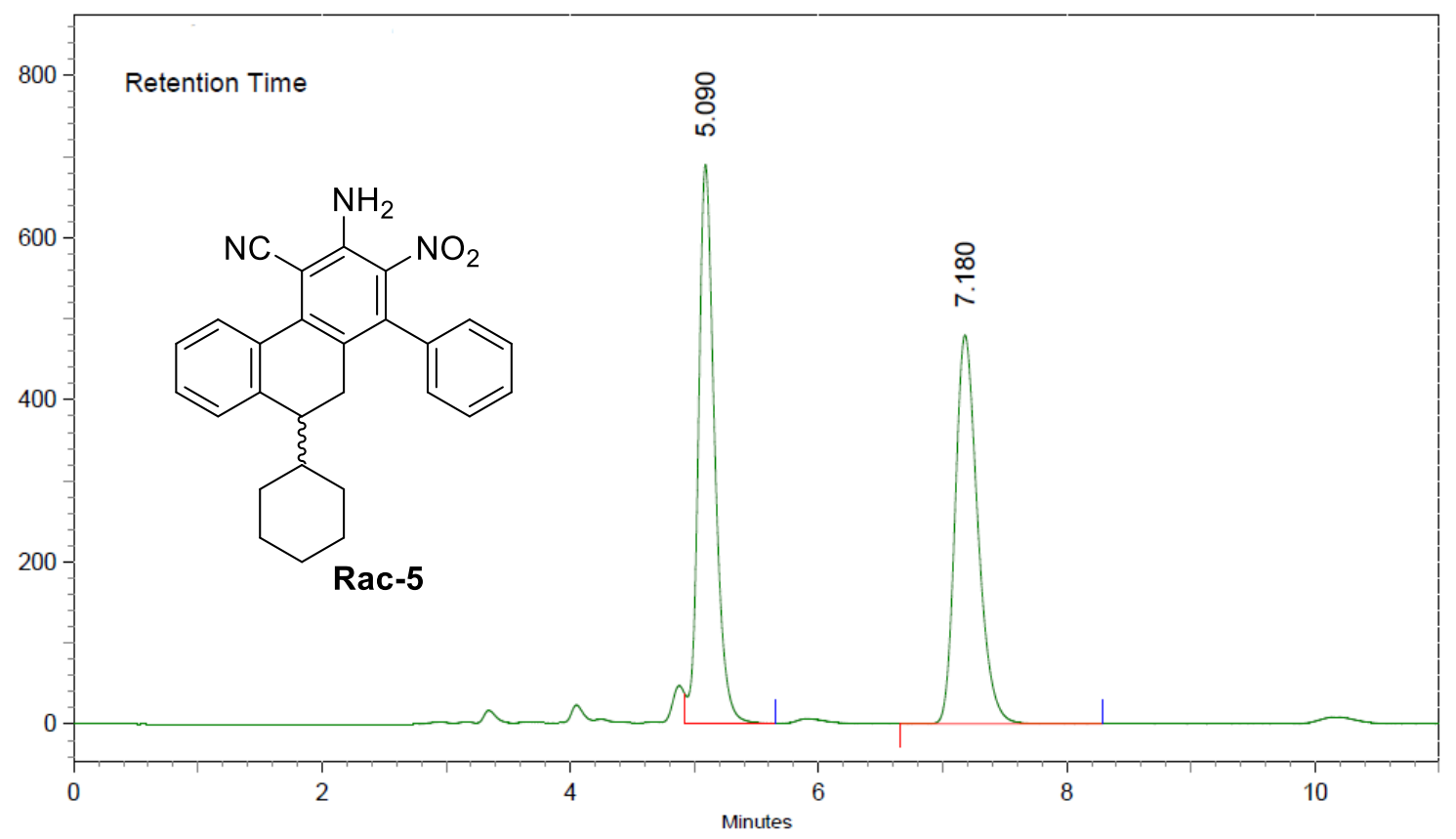

\begin{tabular}{llllll|} 
Peak No. & Ret Time & Width & Height & Area & Area [\%] \\
\hline 1 & 5.090 & 0.736 & 11557120 & 104505883 & 50.6631 \\
2 & 7.180 & 1.627 & 8042169 & 101770050 & 49.3369 \\
\hline Totals & & & & \\
& & & 19599289 & 206275933 & 100.0000 \\
\hline
\end{tabular}

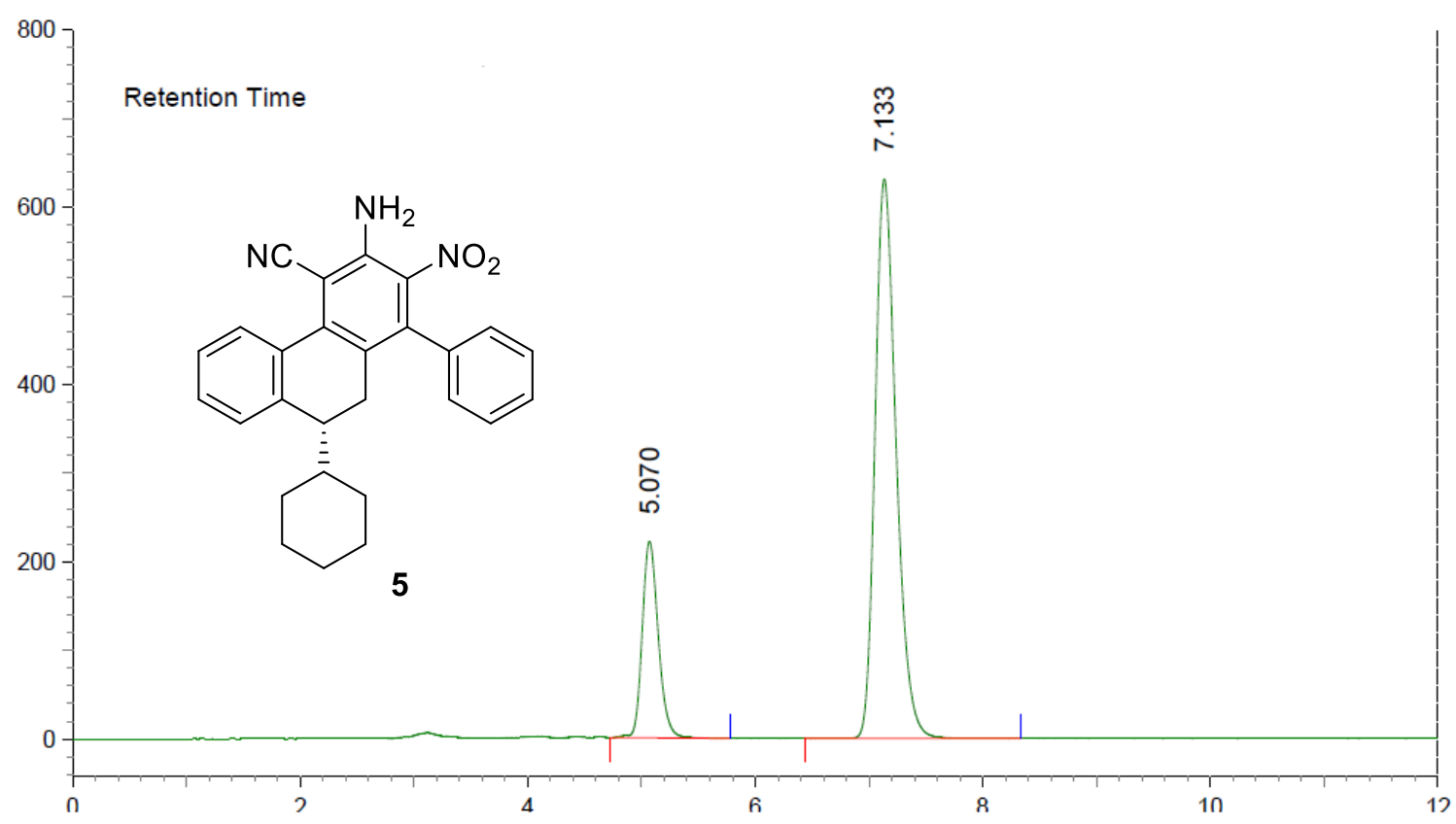

\begin{tabular}{llllll} 
Peak No. & Ret Time & Width & Height & Area & Area [\%] \\
\hline 1 & 5.070 & 1.050 & 3720622 & 36438795 & 21.0414 \\
2 & 7.133 & 1.903 & 10587240 & 136737834 & 78.9586 \\
\hline Totals & & & & \\
& & & 14307862 & 173176629 & 100.0000 \\
\hline
\end{tabular}




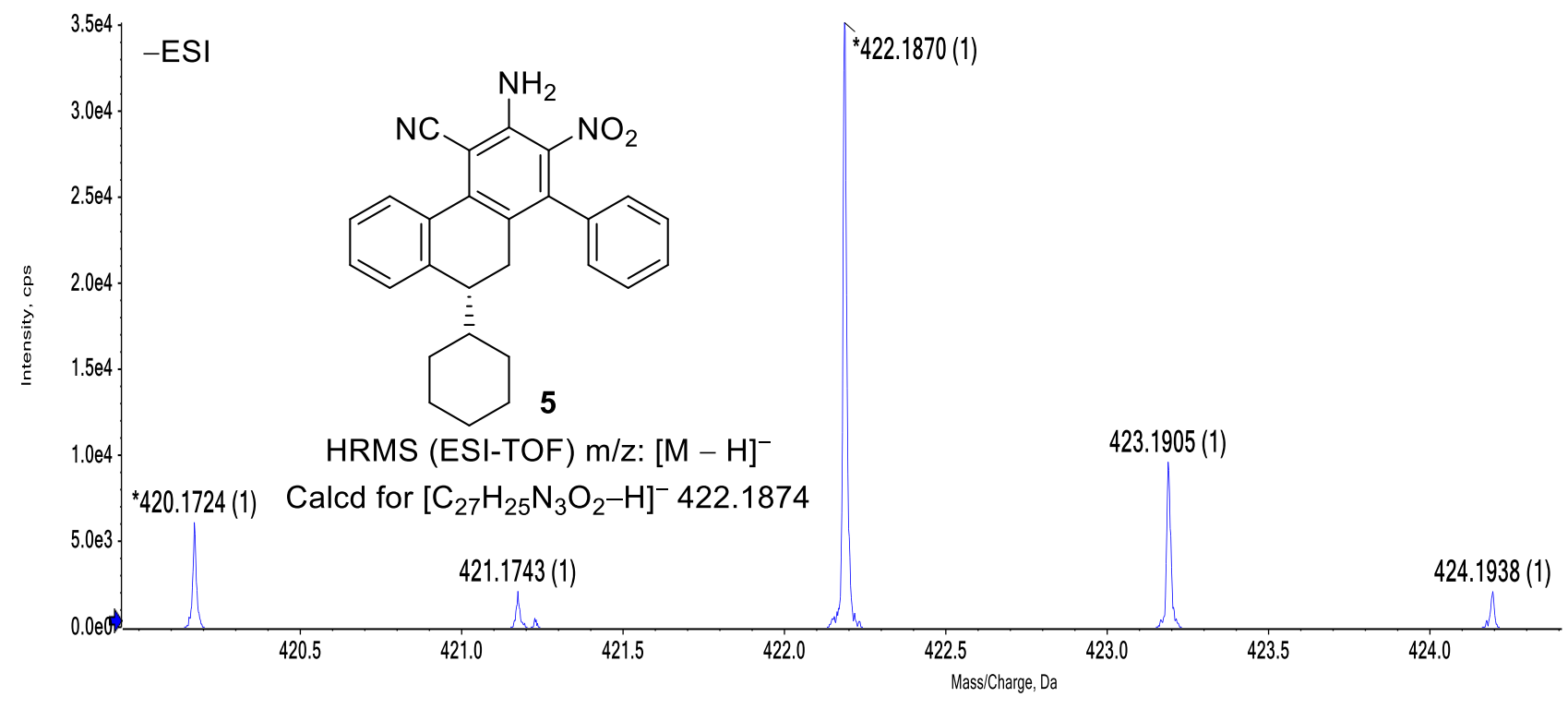




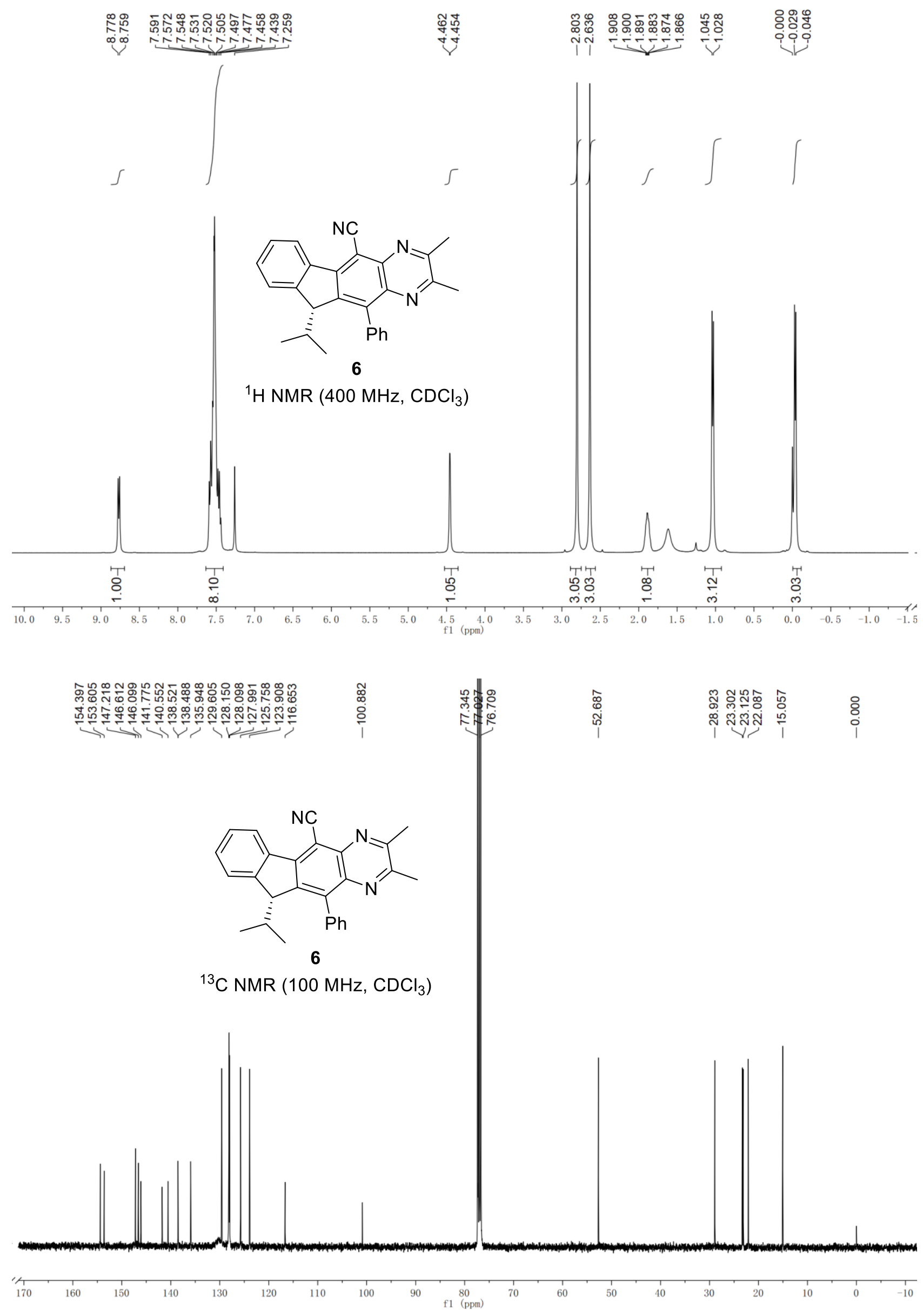




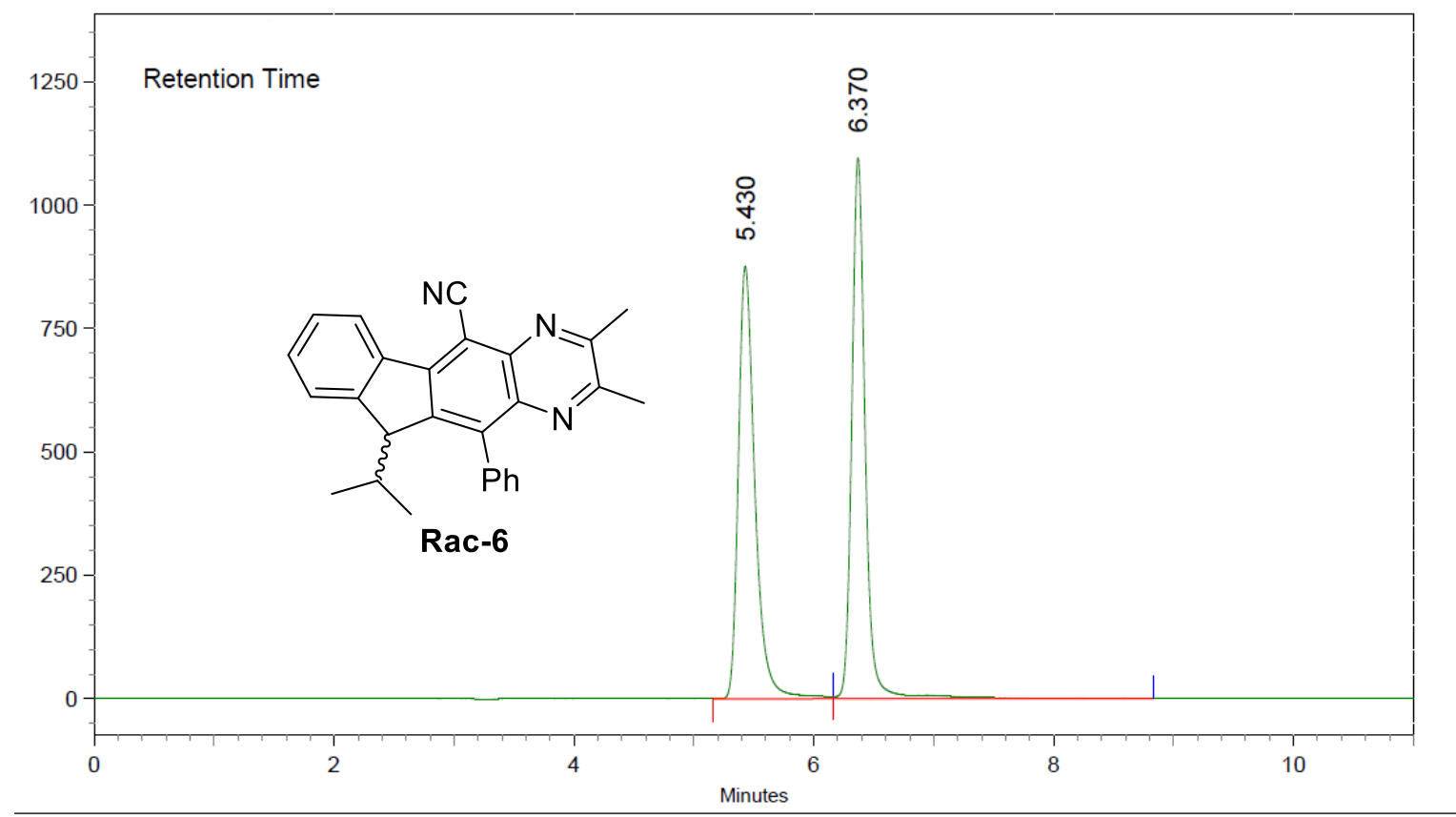

\begin{tabular}{llllll} 
Peak No. & Ret Time & Width & Height & Area & Area [\%] \\
\hline 1 & 5.430 & 1.003 & 14695341 & 141433524 & 49.4711 \\
2 & 6.370 & 2.670 & 18365869 & 144457764 & 50.5289 \\
\hline Totals & & & & \\
& & & 33061210 & 285891288 & 100.0000 \\
\hline
\end{tabular}

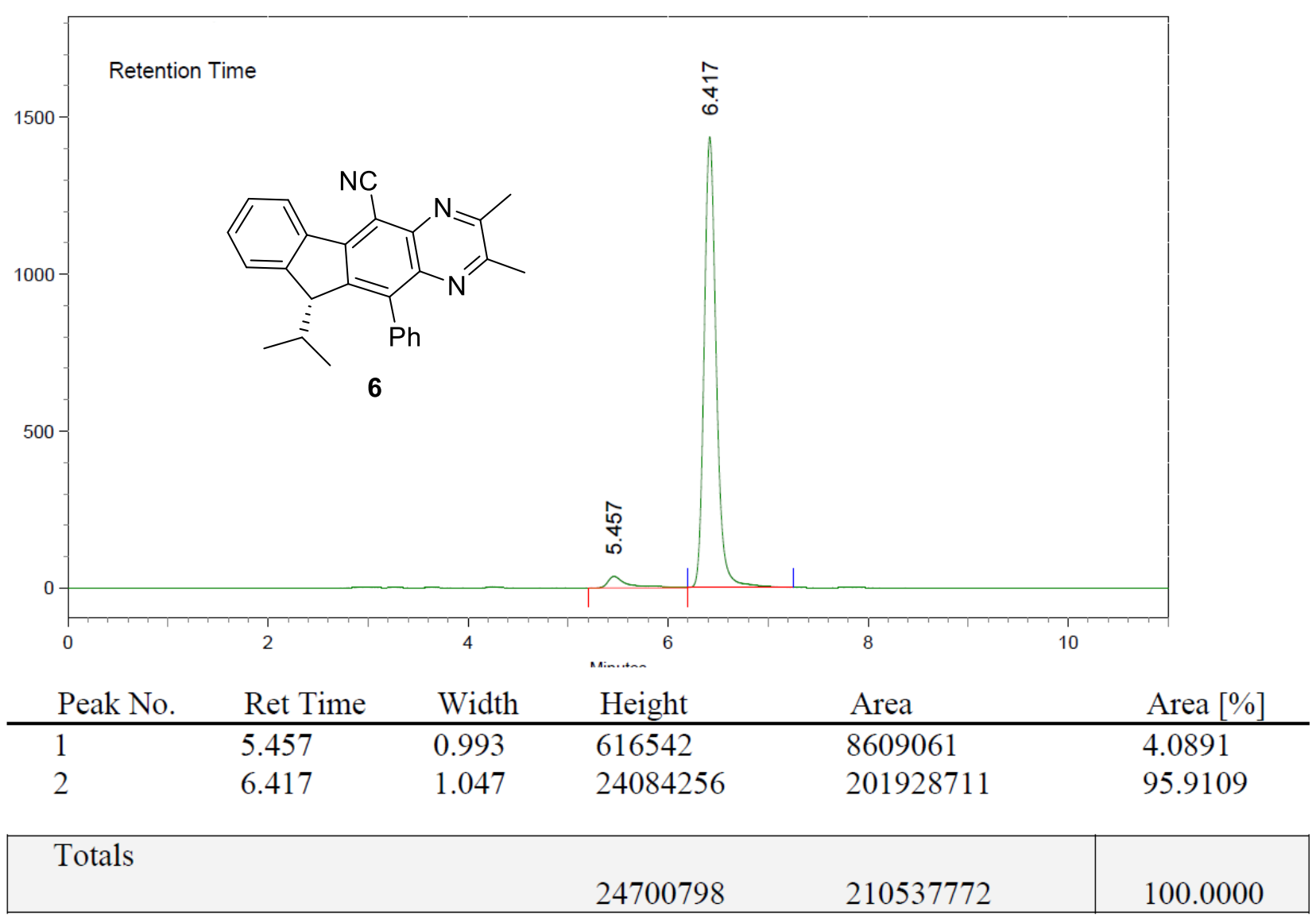




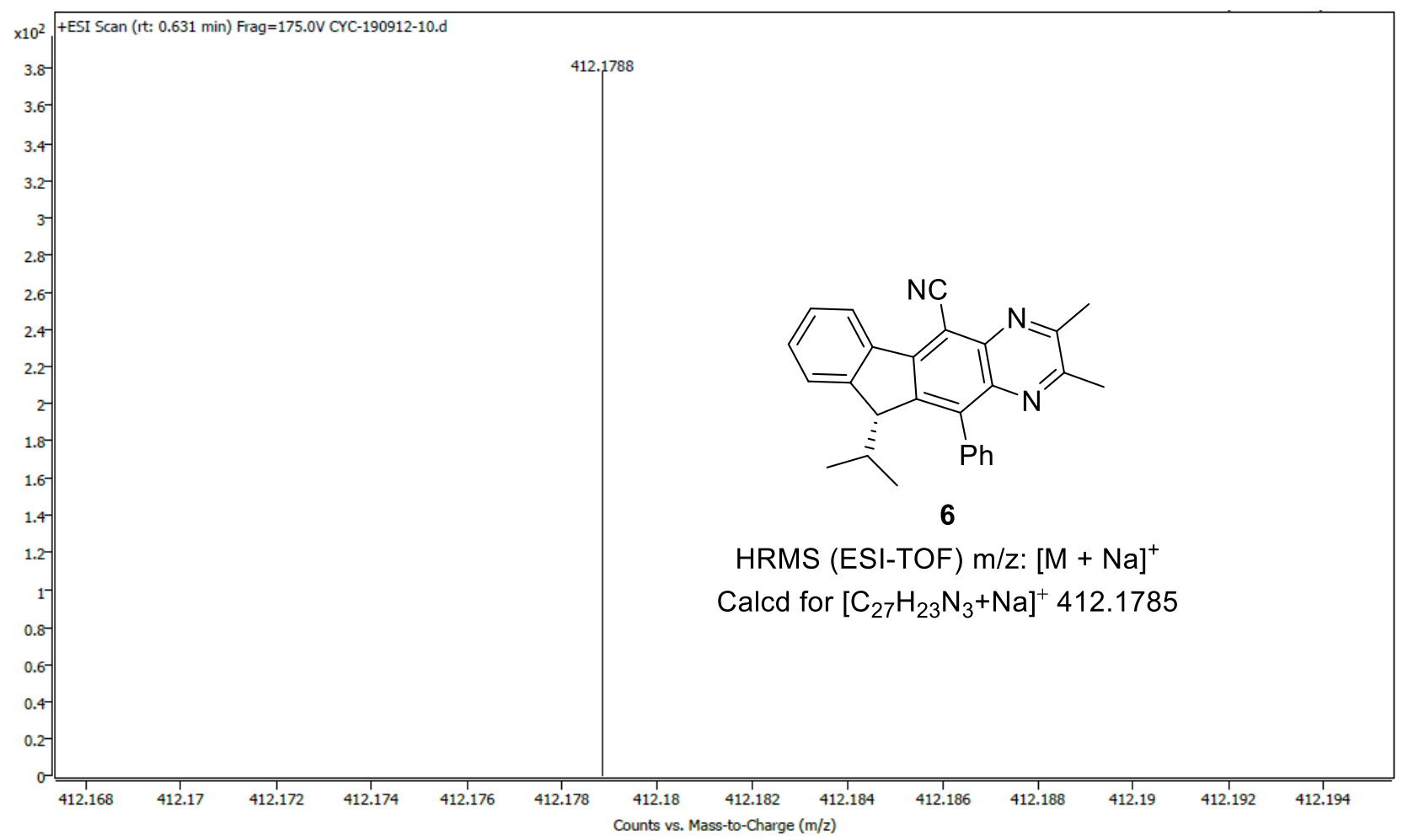

